\title{
Blue Light Induced Difluoroalkylation of Alkynes and
}

Alkenes

Kangkui Li, ${ }^{\ddagger, \S}$ Xuexin Zhang, ${ }^{\ddagger, \S}$ Jingchao Chen, ${ }^{*, \ddagger}$ Yang Gao, ${ }^{\ddagger}$ Chunhui Yang, ${ }^{\ddagger}$ Keyang Zhang, ${ }^{\ddagger}$ Yongyun Zhou ${ }^{\dagger, \ddagger}$ and Baomin Fan*,+,

† School of Chemistry and Environment, Yunnan Minzu University, Yuehua Street, Kunming, 650500, China

‡ Institution Key Laboratory of Chemistry in Ethnic Medicinal Resources (Yunnan Minzu University), State Ethnic Affairs Commission \& Ministry of Education, Kunming, 650500, China

\section{Table of Contents}

1 General information..............................................S2

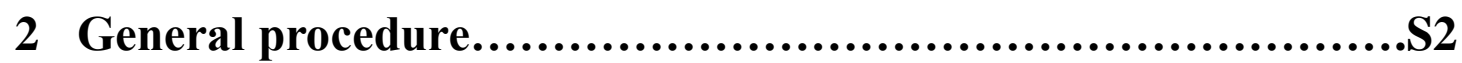

3 Control experiment for mechanistic studies.......................S3

4 Ultraviolet-visible spectroscopy ................................S4

5 Characterization data of iododifluoroalkylation products............S4

6 Characterization data of hydrodifluoroalkylation products......S13

7 Characterization data of difluoroalkylation products............S16

8 Reference......................................................S22

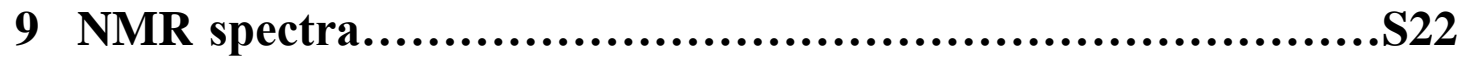




\section{General information}

Unless stated otherwise, all reactions were carried out under argon. ${ }^{1} \mathrm{H}$ NMR spectra were recorded using a Bruker $400 \mathrm{MHz}$ instrument with tetramethylsilane (TMS) as an internal standard. ${ }^{13} \mathrm{C}$ NMR spectra were obtained at $101 \mathrm{MHz}$ and referenced to the internal solvent signals. ${ }^{19} \mathrm{~F}$ NMR spectra were obtained at $376 \mathrm{MHz}$. Commercially available reagents and solvents were used without further purification unless indicated otherwise, $\mathrm{ICF}_{2} \mathrm{COOEt}$ was purchased from J\&K Scientific Ltd.

\section{General procedure}

\section{A. General procedure for the blue light induced difluoroalkylation of alkynes or alkenes}

Under the protection of argon, $\mathrm{Cs}_{2} \mathrm{CO}_{3}(130.3 \mathrm{mg}, 0.4 \mathrm{mmol}, 2.0$ equiv), alkyne or alkene (0.2 mmol), $\mathrm{ICF}_{2} \mathrm{COOEt}$ (100.0 mg, $0.4 \mathrm{mmol}, 2.0$ equiv), solvent $\left(\mathrm{CH}_{3} \mathrm{CN}\right.$ or THF, $1 \mathrm{~mL}$ ) were added sequentially to a $25 \mathrm{~mL}$ Schlenk tube equipped with a magnetic stir bar. The reaction was stirred under $30 \mathrm{~W}$ blue LED irradiation at room temperature, and cooled by a fan to keep the temperature relatively constant. The resulting solution was concentrated in vacuum and the residue was purified by chromatography on silica gel, eluting with the mixture of ethyl acetate/petroleum ether to give difluoroalkylation product, the total yield of $Z$ and $E$-products was recorded in isolated yield. The $Z / E$ ratios are determined by ${ }^{19} \mathrm{~F}$ and ${ }^{1} \mathrm{H}$ NMR spectroscopy.

\section{B. General procedure for the preparation of internal alkynes ${ }^{1}$}

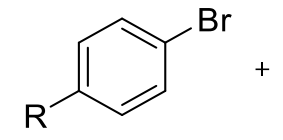

$\mathrm{R}=\mathrm{Me}, \mathrm{MeO}, \mathrm{F}, \mathrm{Cl}$
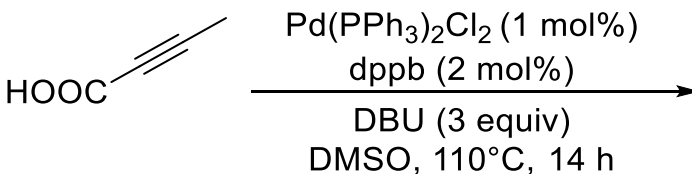

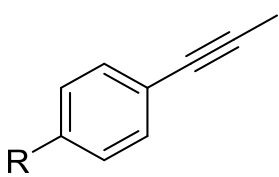

Under $\mathrm{Ar}$ or $\mathrm{N}_{2}$ atmosphere, to a $50 \mathrm{~mL}$ of Schlenk tube equipped with a magnetic stir bar were added $\mathrm{Pd}\left(\mathrm{PPh}_{3}\right)_{2} \mathrm{Cl}_{2}(28.0 \mathrm{mg}, 1 \mathrm{~mol} \%)$, 1,4-bis(diphenylphosphino)butane (dppb) (34.1 mg, 2 mol\%), 2-butynoic acid (420 mg, 5.0 mmol, 1.25 equiv). Then $\operatorname{ArBr}$ (4.0 mmol, 1.0 equiv), DBU (1.79 mL, $12 \mathrm{mmol}, 3.0$ equiv) and DMSO (12 mL) were added respectively. The Schlenk tube was screw capped and heated to $110{ }^{\circ} \mathrm{C}$ (oil bath). After stirring for $14 \mathrm{~h}$ at $110{ }^{\circ} \mathrm{C}$, the reaction mixture was cooled to room temperature, and then sat. $\mathrm{NH}_{4} \mathrm{Cl}$ aq. was poured into the reaction mixture and extracted with $\mathrm{CH}_{2} \mathrm{Cl}_{2}$. The organic layer was washed 
with water, dried over $\mathrm{Na}_{2} \mathrm{SO}_{4}$, and filtered. The filtrate was concentrated in vacuo and the residue was purified by silica gel column chromatography to afford alkynes.

\section{Control experiment for mechanistic studies}

a) Radical trapping experiment with TEMPO $^{2}$

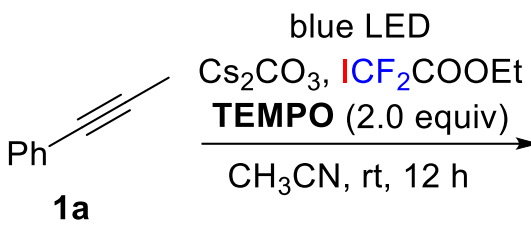

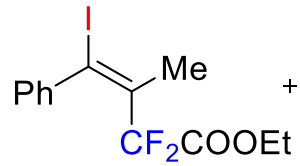

2a, trace

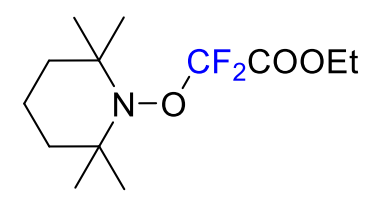

6, $72 \%$ yield

According to general procedure A, using 1a (MW $=116.1,0.2 \mathrm{mmol}, 23.2 \mathrm{mg}$ ) and $\mathrm{ICF}_{2} \mathrm{COOEt}(\mathrm{MW}=250.0,0.4 \mathrm{mmol}, 100.0 \mathrm{mg})$ under standard reaction conditions, 2.0 equiv TEMPO (MW = 156.3, $0.4 \mathrm{mmol}, 62.5 \mathrm{mg}$ ) was added before $\mathrm{ICF}_{2} \mathrm{COOEt}$, stirred for $12 \mathrm{~h}$ under $30 \mathrm{~W}$ blue LED irradiation at room temperature, only a trace amount of the product 2a was detected by GC-MS analysis, the product 6 (TEMPO-CF ${ }_{2} \mathrm{COOEt}$ ) was obtained as main product, and purified by silica gel chromatography $(\mathrm{PE} / \mathrm{EA}=80: 1)$ as faint yellow liquid $(\mathrm{MW}=279.2,80.2 \mathrm{mg}, 0.288 \mathrm{mmol}, 72 \%$ yield $) .{ }^{1} \mathrm{H} \mathrm{NMR}\left(400 \mathrm{MHz}, \mathrm{CDCl}_{3}\right) \delta$ $4.36(\mathrm{q}, J=7.1 \mathrm{~Hz}, 2 \mathrm{H}), 1.64-1.51(\mathrm{~m}, 5 \mathrm{H}), 1.37(\mathrm{t}, J=7.2 \mathrm{~Hz}, 4 \mathrm{H}), 1.19(\mathrm{dd}, J=7.9,5.3$ $\mathrm{Hz}, 12 \mathrm{H}) .{ }^{19} \mathrm{~F} \mathrm{NMR}\left(376 \mathrm{MHz}, \mathrm{CDCl}_{3}\right) \delta-73.50$.

b) Control experiments with product 2a

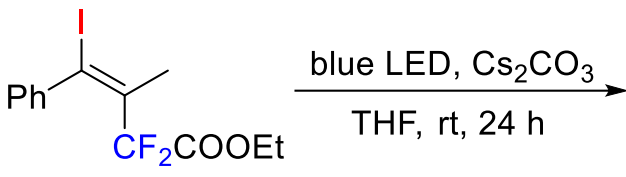

2a $(E / Z=87: 13)$

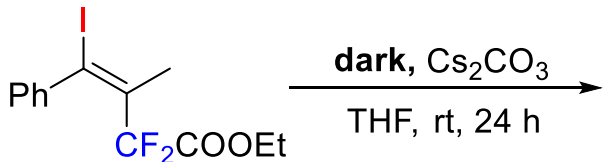

2a $(E / Z=87: 13)$<smiles>CCOC(=O)C(C)(F)C(C)=Cc1ccccc1</smiles>

$3 a, 77 \%$ yield, $E / Z=46: 54$<smiles>CCOC(=O)C(C)(F)C(C)=Cc1ccccc1</smiles>

3a, trace

According to general procedure $\mathbf{A}$, the above experiments were performed, $\mathrm{Cs}_{2} \mathrm{CO}_{3}(\mathrm{MW}=$ 325.8, $0.4 \mathrm{mmol}, 130.3 \mathrm{mg})$ and 2a $(\mathrm{MW}=366.0,0.2 \mathrm{mmol}, 73.2 \mathrm{mg}, E / \mathrm{Z}=87: 13)$ were add to THF ( $1 \mathrm{~mL})$, stirred for $24 \mathrm{~h}$ at room temperature. When rection under $30 \mathrm{~W}$ blue LED irradiation (eqn(1)), product 3a was purified by silica gel chromatography $(\mathrm{PE} / \mathrm{EA}=60: 1)$ as colorless liquid $(\mathrm{MW}=240.1,37.0 \mathrm{mg}, 0.154 \mathrm{mmol}, 77 \%$ yield, $E / Z=46: 54)$. When reaction in dark (eqn(2)), only a trace amount of the product 3a was detected by GC-MS analysis. 
c) Deuterium labeling experiment with THF- $d_{8}$

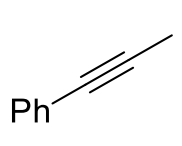

$1 \mathrm{a}$

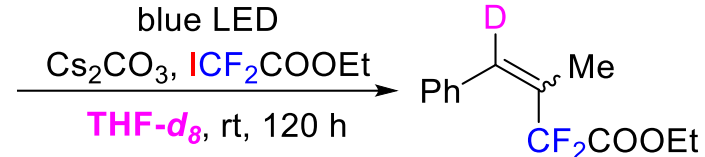

3a-D, $51 \%$ yield, $80 \% \mathrm{D}, E / Z=53: 47$

According to general procedure A, using 1a $(\mathrm{MW}=116.1,0.2 \mathrm{mmol}, 23.2 \mathrm{mg}$ ) and $\mathrm{ICF}_{2} \mathrm{COOEt}(\mathrm{MW}=250.0,0.4 \mathrm{mmol}, 100.0 \mathrm{mg})$ under standard reaction conditions, using THF- $d_{8}$ instead of THF, the deuteride product 3a-D was purified by silica gel chromatography $(\mathrm{PE} / \mathrm{EA}=60: 1)$ as colorless liquid $(\mathrm{MW}=241.1,24.6 \mathrm{mg}, 0.102 \mathrm{mmol}, 51 \%$ yield, $80 \% \mathrm{D}$ incorporated, $E / Z=53: 47) .{ }^{1} \mathrm{H}$ NMR $\left(400 \mathrm{MHz}, \mathrm{CDCl}_{3}\right) \delta 7.36-7.21(\mathrm{~m}, 10 \mathrm{H}), 4.36(\mathrm{q}, J=$ $7.1 \mathrm{~Hz}, 2 \mathrm{H}), 3.91-3.85(\mathrm{~m}, 2 \mathrm{H}), 2.06(\mathrm{~s}, 3 \mathrm{H}), 1.99(\mathrm{~s}, 3 \mathrm{H}), 1.36(\mathrm{t}, J=7.1 \mathrm{~Hz}, 3 \mathrm{H}), 1.12(\mathrm{t}, J$ $=7.2 \mathrm{~Hz}, 3 \mathrm{H})$. HRMS (EI) calcd. for $\mathrm{C}_{13} \mathrm{H}_{13} \mathrm{DF}_{2} \mathrm{O}_{2}{ }^{+}\left(\mathrm{M}^{+}\right)$241.1019, found 241.1026 .

\section{Ultraviolet-visible spectroscopy}

A strong absorption signal of $\mathrm{ICF}_{2} \mathrm{COOEt}\left(1.0 \mathrm{mmol} / \mathrm{L}\right.$ in $\left.\mathrm{CH}_{3} \mathrm{CN}\right)$ and the mixture of $\mathrm{ICF}_{2} \mathrm{COOEt}$ and $1 \mathrm{a}$ were observed at $360 \mathrm{~nm}$. In contrast, no absorption signal was observed by the solution of $\mathbf{1 a}$ along.

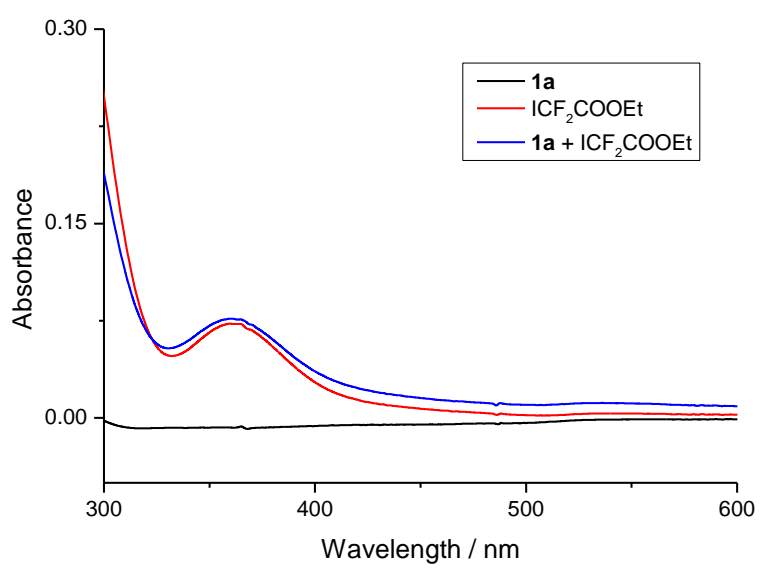

\section{Characterization data of iododifluoroalkylation products}

ethyl $(E)$-2,2-difluoro-4-iodo-3-methyl-4-phenylbut-3-enoate ${ }^{2}$<smiles>CCOC(=O)C(C)=C(I)c1ccccc1</smiles>

2a 
Following general procedure $\mathbf{A}, \mathrm{Cs}_{2} \mathrm{CO}_{3}(\mathrm{MW}=325.8,0.4 \mathrm{mmol}, 130.3 \mathrm{mg}), \mathbf{1 a}(\mathrm{MW}=$ $116.1,0.2 \mathrm{mmol}, 23.2 \mathrm{mg}$ ) and $\mathrm{ICF}_{2} \mathrm{COOEt}(\mathrm{MW}=250.0,0.4 \mathrm{mmol}, 100.0 \mathrm{mg}$ ) were added to $\mathrm{CH}_{3} \mathrm{CN}(1 \mathrm{~mL})$, stirred for $10 \mathrm{~h}$ under $30 \mathrm{~W}$ blue LED irradiation at room temperature, the reaction mixture was purified by silica gel chromatography $(\mathrm{PE} / \mathrm{EA}=60: 1)$ to obtain product 2a as colorless liquid ( $\mathrm{MW}=366.0,65.3 \mathrm{mg}, 0.178 \mathrm{mmol}, 89 \%$ yield, $E / Z=87: 13$ ).

${ }^{1} \mathrm{H}$ NMR (400 MHz, $\left.\mathrm{CDCl}_{3}\right) \delta 7.25(\mathrm{dtd}, J=15.7,8.1,6.5 \mathrm{~Hz}, 5 \mathrm{H}), 3.91(\mathrm{q}, J=7.2 \mathrm{~Hz}, 2 \mathrm{H})$, $2.28(\mathrm{~s}, 3 \mathrm{H}), 1.19(\mathrm{t}, J=7.2 \mathrm{~Hz}, 3 \mathrm{H})$.

${ }^{13} \mathrm{C}$ NMR $\left(101 \mathrm{MHz}, \mathrm{CDCl}_{3}\right) \delta 163.2,162.9,162.5,142.9,135.6,135.4,135.2,128.7,128.4$, 127.8, 113.2, 110.7, 109.0, 63.0, 25.4, 13.7 .

${ }^{19} \mathrm{~F}$ NMR $\left(376 \mathrm{MHz}, \mathrm{CDCl}_{3}\right) \delta-93.89(E),-99.68(Z)$.

\section{(E)-1-(3-(ethylperoxy)-3,3-difluoro-1-iodo-2-methylprop-1-en-1-yl)-4-methylbenz}

ene<smiles>CCOC(=O)C(C)=C(I)c1ccc(C)cc1</smiles>

2b

Following general procedure $\mathbf{A}, \mathrm{Cs}_{2} \mathrm{CO}_{3}(\mathrm{MW}=325.8,0.4 \mathrm{mmol}, 130.3 \mathrm{mg}), \mathbf{1 b}(\mathrm{MW}=$ $130.1,0.2 \mathrm{mmol}, 26.0 \mathrm{mg}$ ) and $\mathrm{ICF}_{2} \mathrm{COOEt}(\mathrm{MW}=250.0,0.4 \mathrm{mmol}, 100.0 \mathrm{mg}$ ) were added to $\mathrm{CH}_{3} \mathrm{CN}(1 \mathrm{~mL})$, stirred for $20 \mathrm{~h}$ under $30 \mathrm{~W}$ blue LED irradiation at room temperature, the reaction mixture was purified by silica gel chromatography $(\mathrm{PE} / \mathrm{EA}=60: 1)$ to obtain product 2b as colorless liquid (MW =380.0, 70.0mg, $0.184 \mathrm{mmol}, 92 \%$ yield, $E / Z=80: 20$ ).

${ }^{1} \mathrm{H}$ NMR (400 MHz, $\left.\mathrm{CDCl}_{3}\right) \delta 7.10(\mathrm{~s}, 4 \mathrm{H}), 3.90(\mathrm{q}, J=7.2 \mathrm{~Hz}, 2 \mathrm{H}), 2.33(\mathrm{~s}, 3 \mathrm{H}), 2.27(\mathrm{~s}$, $3 \mathrm{H}), 1.19(\mathrm{t}, J=7.2 \mathrm{~Hz}, 3 \mathrm{H})$.

${ }^{13} \mathrm{C}$ NMR $\left(101 \mathrm{MHz}, \mathrm{CDCl}_{3}\right) \delta 162.9,141.3,140.0,138.8,138.4,135.5,135.3,135.0,129.1$, 128.4, 127.7, 113.1, 110.6, 109.5, 108.1, 63.0, 25.4, 21.3, 17.7, 14.0, 13.7.

${ }^{19} \mathrm{~F}$ NMR $\left(376 \mathrm{MHz}, \mathrm{CDCl}_{3}\right) \delta-93.62(E),-99.54(Z)$.

HRMS (EI) calcd. for $\mathrm{C}_{14} \mathrm{H}_{15} \mathrm{~F}_{2} \mathrm{IO}_{2}{ }^{+}\left(\mathrm{M}^{+}\right) 380.0079$, found 380.0091 .

\section{(E)-4,4-difluoro-6-iodo-6-(4-methoxyphenyl)-5-methylhex-5-en-3-one}<smiles>CCOC(=O)C(C)=C(I)c1ccc(OC)cc1</smiles>

Following general procedure $\mathbf{A}, \mathrm{Cs}_{2} \mathrm{CO}_{3}(\mathrm{MW}=325.8,0.4 \mathrm{mmol}, 130.3 \mathrm{mg}), \mathbf{1 c}(\mathrm{MW}=$ $146.1,0.2 \mathrm{mmol}, 29.2 \mathrm{mg}$ ) and $\mathrm{ICF}_{2} \mathrm{COOEt}(\mathrm{MW}=250.0,0.4 \mathrm{mmol}, 100.0 \mathrm{mg}$ ) were added to $\mathrm{CH}_{3} \mathrm{CN}(1 \mathrm{~mL})$, stirred for $15 \mathrm{~h}$ under $30 \mathrm{~W}$ blue LED irradiation at room temperature, the reaction mixture was purified by silica gel chromatography $(\mathrm{PE} / \mathrm{EA}=30: 1)$ to obtain product 2c as colorless liquid ( $\mathrm{MW}=396.0,64.7 \mathrm{mg}, 0.163 \mathrm{mmol}, 82 \%$ yield, $E / Z=80: 20$ ).

${ }^{1} \mathrm{H}$ NMR $\left(400 \mathrm{MHz}, \mathrm{CDCl}_{3}\right) \delta 7.15(\mathrm{~d}, J=8.7 \mathrm{~Hz}, 2 \mathrm{H}), 6.80(\mathrm{~d}, J=8.8 \mathrm{~Hz}, 2 \mathrm{H}), 3.91(\mathrm{q}, J=$ $7.2 \mathrm{~Hz}, 2 \mathrm{H}), 3.79$ (s, 3H), 2.26 (s, 3H), 1.19 (t, $J=7.2 \mathrm{~Hz}, 3 \mathrm{H}$ ). 
${ }^{13} \mathrm{C}$ NMR $\left(101 \mathrm{MHz}, \mathrm{CDCl}_{3}\right) \delta 163.2,162.9,162.6,159.7,135.7,135.4,135.2,130.2,113.2$, 110.6, 109.5, 62.9, 55.3, 25.3, 13.7.

${ }^{19} \mathrm{~F} \mathrm{NMR}\left(376 \mathrm{MHz}, \mathrm{CDCl}_{3}\right) \delta-93.26(E),-99.44(Z)$.

HRMS (EI) calcd. for $\mathrm{C}_{14} \mathrm{H}_{15} \mathrm{~F}_{2} \mathrm{IO}_{3}{ }^{+}\left(\mathrm{M}^{+}\right) 396.0028$, found 396.0042.

(E)-1-(3-(ethylperoxy)-3,3-difluoro-1-iodo-2-methylprop-1-en-1-yl)-4-fluorobenze

ne<smiles>CCOC(=O)C(C)=C(I)c1ccc(F)cc1</smiles>

2d

Following general procedure $\mathbf{A}, \mathrm{Cs}_{2} \mathrm{CO}_{3}(\mathrm{MW}=325.8,0.4 \mathrm{mmol}, 130.3 \mathrm{mg}), \mathbf{1 d}(\mathrm{MW}=$ 134.1, $0.2 \mathrm{mmol}, 26.8 \mathrm{mg}$ ) and $\mathrm{ICF}_{2} \mathrm{COOEt}(\mathrm{MW}=250.0,0.4 \mathrm{mmol}, 100.0 \mathrm{mg}$ ) were added to $\mathrm{CH}_{3} \mathrm{CN}(1 \mathrm{~mL})$, stirred for $13 \mathrm{~h}$ under $30 \mathrm{~W}$ blue LED irradiation at room temperature, the reaction mixture was purified by silica gel chromatography $(\mathrm{PE} / \mathrm{EA}=60: 1)$ to obtain product 2d as colorless liquid ( $\mathrm{MW}=384.0,69.1 \mathrm{mg}, 0.180 \mathrm{mmol}, 90 \%$ yield, $E / Z=85: 15$ ).

${ }^{1} \mathrm{H} \mathrm{NMR}\left(400 \mathrm{MHz}, \mathrm{CDCl}_{3}\right) \delta 7.22-7.17(\mathrm{~m}, 2 \mathrm{H}), 6.98(\mathrm{t}, J=8.7 \mathrm{~Hz}, 2 \mathrm{H}), 4.00(\mathrm{q}, J=7.2$ $\mathrm{Hz}, 2 \mathrm{H}), 2.26(\mathrm{~s}, 3 \mathrm{H}), 1.23(\mathrm{t}, J=7.2 \mathrm{~Hz}, 3 \mathrm{H})$.

${ }^{13} \mathrm{C}$ NMR $\left(101 \mathrm{MHz}, \mathrm{CDCl}_{3}\right) \delta 163.7,163.2,162.9,162.5,161.2,139.1,136.1,130.3,115.0$, 114.8, 110.7, 107.6, 63.1, 25.5, 13.7.

${ }^{19} \mathrm{~F}$ NMR (376 MHz, $\left.\mathrm{CDCl}_{3}\right) \delta$-94.25 (E), -99.77 (Z), -111.84 (E), -112.42 (Z).

HRMS (EI) calcd. for $\mathrm{C}_{13} \mathrm{H}_{12} \mathrm{~F}_{3} \mathrm{IO}_{2}{ }^{+}\left(\mathrm{M}^{+}\right)$383.9829, found 383.9836 .

ethyl (E)-4-(4-chlorophenyl)-2,2-difluoro-4-iodo-3-methylbut-3-enoate<smiles>CCOC(=O)C(C)=C(C)c1ccc(Cl)cc1</smiles>

Following general procedure $\mathbf{A}, \mathrm{Cs}_{2} \mathrm{CO}_{3}(\mathrm{MW}=325.8,0.4 \mathrm{mmol}, 130.3 \mathrm{mg}), \mathbf{1 e}(\mathrm{MW}=$ $150.0,0.2 \mathrm{mmol}, 30.0 \mathrm{mg}$ ) and $\mathrm{ICF}_{2} \mathrm{COOEt}(\mathrm{MW}=250.0,0.4 \mathrm{mmol}, 100.0 \mathrm{mg}$ ) were added to $\mathrm{CH}_{3} \mathrm{CN}(1 \mathrm{~mL})$, stirred for $16 \mathrm{~h}$ under $30 \mathrm{~W}$ blue LED irradiation at room temperature, the reaction mixture was purified by silica gel chromatography $(\mathrm{PE} / \mathrm{EA}=60: 1)$ to obtain product 2e as colorless liquid (MW = 400.0, $74.4 \mathrm{mg}, 0.186 \mathrm{mmol}, 93 \%$ yield, $E / Z=78: 22$ ).

${ }^{1} \mathrm{H}$ NMR (400 MHz, $\left.\mathrm{CDCl}_{3}\right) \delta 7.20(\mathrm{~d}, J=8.5 \mathrm{~Hz}, 2 \mathrm{H}), 7.08(\mathrm{~d}, J=8.5 \mathrm{~Hz}, 2 \mathrm{H}), 3.93(\mathrm{q}, J=$ $7.2 \mathrm{~Hz}, 2 \mathrm{H}), 2.18$ (s, 3H), 1.16 (t, $J=7.2 \mathrm{~Hz}, 3 \mathrm{H})$.

${ }^{13} \mathrm{C}$ NMR $\left(101 \mathrm{MHz}, \mathrm{CDCl}_{3}\right) \delta 163.2,162.9,162.6,141.4,136.3,136.0,135.8,134.6,134.3$, 129.6, 129.2, 128.8, 128.1, 113.3, 110.8, 108.2, 107.3, 63.2, 29.8, 25.5, 17.8, 13.7.

${ }^{19} \mathrm{~F}$ NMR (376 MHz, $\left.\mathrm{CDCl}_{3}\right) \delta-94.49(E),-99.83(Z)$.

HRMS (EI) calcd. for $\mathrm{C}_{13} \mathrm{H}_{12} \mathrm{ClF}_{2} \mathrm{IO}_{2}^{+}\left(\mathrm{M}^{+}\right)$399.9533, found 399.9540.

\section{(E)-4,4-difluoro-5-(iodo(phenyl)methylene)heptan-3-one ${ }^{1}$}


<smiles>CCOC(=O)C(F)(F)/C(CC)=C(\I)c1ccccc1</smiles>

Following general procedure $\mathbf{A}, \mathrm{Cs}_{2} \mathrm{CO}_{3}(\mathrm{MW}=325.8,0.4 \mathrm{mmol}, 130.3 \mathrm{mg})$, 1f $(\mathrm{MW}=$ $130.1,0.2 \mathrm{mmol}, 26.0 \mathrm{mg}$ ) and $\mathrm{ICF}_{2} \mathrm{COOEt}(\mathrm{MW}=250.0,0.4 \mathrm{mmol}, 100.0 \mathrm{mg}$ ) were added to $\mathrm{CH}_{3} \mathrm{CN}(1 \mathrm{~mL})$, stirred for $16 \mathrm{~h}$ under $30 \mathrm{~W}$ blue LED irradiation at room temperature, the reaction mixture was purified by silica gel chromatography $(\mathrm{PE} / \mathrm{EA}=60: 1)$ to obtain product 2f as colorless liquid (MW $=380.0,65.5 \mathrm{mg}, 0.172 \mathrm{mmol}, 86 \%$ yield, $E / Z=85: 15$ ).

${ }^{1} \mathrm{H}$ NMR (400 MHz, $\left.\mathrm{CDCl}_{3}\right) \delta 7.30-7.17(\mathrm{~m}, 5 \mathrm{H}), 3.86(\mathrm{q}, J=7.2 \mathrm{~Hz}, 2 \mathrm{H}), 2.68$ (qd, $J=7.5$, $6.2 \mathrm{~Hz}, 2 \mathrm{H}), 1.22(\mathrm{t}, J=7.5 \mathrm{~Hz}, 3 \mathrm{H}), 1.17(\mathrm{t}, J=7.2 \mathrm{~Hz}, 3 \mathrm{H})$.

${ }^{13} \mathrm{C}$ NMR $\left(101 \mathrm{MHz}, \mathrm{CDCl}_{3}\right) \delta 163.2,162.9,162.6,142.6,141.1,128.8,128.5,127.9,110.8$, 108.3, 62.9, 32.3, 13.6, 12.4 .

${ }^{19} \mathrm{~F}$ NMR (376 MHz, $\left.\mathrm{CDCl}_{3}\right) \delta-92.55(E),-99.11(Z)$.

\section{ethyl (E)-2,2-difluoro-4-iodo-3-pentylnon-3-enoate}<smiles>CCCCC/C(I)=C(\CCCCC)C(F)(F)F</smiles>

Following general procedure $\mathbf{A}, \mathrm{Cs}_{2} \mathrm{CO}_{3}(\mathrm{MW}=325.8,0.4 \mathrm{mmol}, 130.3 \mathrm{mg}), \mathbf{1 g}(\mathrm{MW}=$ $166.2,0.2 \mathrm{mmol}, 33.2 \mathrm{mg}$ ) and $\mathrm{ICF}_{2} \mathrm{COOEt}(\mathrm{MW}=250.0,0.4 \mathrm{mmol}, 100.0 \mathrm{mg}$ ) were added to $\mathrm{CH}_{3} \mathrm{CN}(1 \mathrm{~mL})$, stirred for $12 \mathrm{~h}$ under $30 \mathrm{~W}$ blue LED irradiation at room temperature, the reaction mixture was purified by silica gel chromatography $(\mathrm{PE} / \mathrm{EA}=60: 1)$ to obtain product $2 \mathrm{~g}$ as colorless liquid (MW $=416.1,64.7 \mathrm{mg}, 0.156 \mathrm{mmol}, 78 \%$ yield, $E / Z=86: 14$ ).

${ }^{1} \mathrm{H}$ NMR (400 MHz, $\left.\mathrm{CDCl}_{3}\right) \delta 4.31(\mathrm{q}, J=7.1 \mathrm{~Hz}, 2 \mathrm{H}), 2.66(\mathrm{dd}, J=10.6,4.7 \mathrm{~Hz}, 2 \mathrm{H}), 2.51$ $-2.28(\mathrm{~m}, 2 \mathrm{H}), 1.51(\mathrm{ddd}, J=14.6,11.6,7.2 \mathrm{~Hz}, 4 \mathrm{H}), 1.34(\mathrm{td}, J=7.3,1.9 \mathrm{~Hz}, 11 \mathrm{H}), 0.90(\mathrm{td}$, $J=7.0,4.5 \mathrm{~Hz}, 6 \mathrm{H})$.

${ }^{13} \mathrm{C}$ NMR $\left(101 \mathrm{MHz}, \mathrm{CDCl}_{3}\right) \delta 164.1,163.7,163.4,136.9,136.6,136.4,119.7,114.5,63.2$, 42.7, 39.3, 31.7, 30.7, 30.2, 27.7, 22.4, 18.7, $14.1-13.8$.

${ }^{19} \mathrm{~F}$ NMR $\left(376 \mathrm{MHz}, \mathrm{CDCl}_{3}\right) \delta-96.58(E),-97.98(Z)$.

HRMS (ESI) calcd. for $\mathrm{C}_{16} \mathrm{H}_{27} \mathrm{~F}_{2} \mathrm{INaO}_{2}{ }^{+}\left(\mathrm{M}+\mathrm{Na}^{+}\right) 439.0916$, found 439.0925

\section{1-ethyl 4-methyl (E)-2,2-difluoro-3-(iodo(phenyl)methylene)succinate}<smiles>CCOC(=O)C(C(=O)OCC)=C(I)c1ccccc1</smiles>

$2 \mathrm{~h}$

Following general procedure $\mathbf{A}, \mathrm{Cs}_{2} \mathrm{CO}_{3}(\mathrm{MW}=325.8,0.4 \mathrm{mmol}, 130.3 \mathrm{mg}), \mathbf{1 h}(\mathrm{MW}=$ $160.1,0.2 \mathrm{mmol}, 32.0 \mathrm{mg}$ ) and $\mathrm{ICF}_{2} \mathrm{COOEt}(\mathrm{MW}=250.0,0.4 \mathrm{mmol}, 100.0 \mathrm{mg}$ ) were added to $\mathrm{CH}_{3} \mathrm{CN}(1 \mathrm{~mL})$, stirred for $20 \mathrm{~h}$ under $30 \mathrm{~W}$ blue LED irradiation at room temperature, the reaction mixture was purified by silica gel chromatography $(\mathrm{PE} / \mathrm{EA}=20: 1)$ to obtain product 
2h as colorless liquid (MW $=410.0,45.2 \mathrm{mg}, 0.110 \mathrm{mmol}, 55 \%$ yield, $E / Z=85: 15$ ).

${ }^{1} \mathrm{H}$ NMR (400 MHz, CDCl 3 ) $\delta 7.32(\mathrm{dt}, J=9.5,5.9 \mathrm{~Hz}, 5 \mathrm{H}), 3.99(\mathrm{q}, J=7.2 \mathrm{~Hz}, 2 \mathrm{H}), 3.93(\mathrm{~s}$, $3 \mathrm{H}), 1.21(\mathrm{t}, J=7.2 \mathrm{~Hz}, 3 \mathrm{H})$.

${ }^{13} \mathrm{C}$ NMR $\left(101 \mathrm{MHz}, \mathrm{CDCl}_{3}\right) \delta 164.9,161.9,161.5,161.2,140.8,136.1,129.7,128.3,128.1$, 127.5, 127.1, 112.3, 110.4, 109.8, 107.3, 63.5, 53.3, 13.7.

${ }^{19} \mathrm{~F}$ NMR (376 MHz, $\left.\mathrm{CDCl}_{3}\right) \delta-92.72(E),-99.09(Z)$.

HRMS (ESI) calcd. for $\mathrm{C}_{14} \mathrm{H}_{13} \mathrm{~F}_{2} \mathrm{INaO}_{4}^{+}\left(\mathrm{M}+\mathrm{Na}^{+}\right) 432.9719$, found 432.9718 .

\section{(E)-(3-(ethylperoxy)-3,3-difluoro-1-iodoprop-1-ene-1,2-diyl)dibenzene}<smiles>CCOC(=O)C(=C(I)c1ccccc1)c1ccccc1</smiles>

2i

Following general procedure $\mathbf{A}, \mathrm{Cs}_{2} \mathrm{CO}_{3}(\mathrm{MW}=325.8,0.4 \mathrm{mmol}, 130.3 \mathrm{mg}), \mathbf{1 i}(\mathrm{MW}=178.1$, $0.2 \mathrm{mmol}, 35.6 \mathrm{mg}$ ) and $\mathrm{ICF}_{2} \mathrm{COOEt}(\mathrm{MW}=250.0,0.4 \mathrm{mmol}, 100.0 \mathrm{mg}$ ) were added to $\mathrm{CH}_{3} \mathrm{CN}(1 \mathrm{~mL})$, stirred for $20 \mathrm{~h}$ under $30 \mathrm{~W}$ blue LED irradiation at room temperature, the reaction mixture was purified by silica gel chromatography $(\mathrm{PE} / \mathrm{EA}=50: 1)$ to obtain product $2 \mathbf{i}$ as white solid ( $\mathrm{MW}=428.0,53.9 \mathrm{mg}, 0.126 \mathrm{mmol}, 63 \%$ yield, $E / \mathrm{Z}=85: 15$, m.p. $89-91^{\circ} \mathrm{C}$ ). ${ }^{1} \mathrm{H}$ NMR (400 MHz, CDCl $)$ ) $7.55-7.27(\mathrm{~m}, 10 \mathrm{H}), 3.90(\mathrm{q}, J=7.2 \mathrm{~Hz}, 2 \mathrm{H}), 1.17$ (t, $J=7.2$ $\mathrm{Hz}, 3 \mathrm{H})$.

${ }^{13} \mathrm{C}$ NMR $\left(101 \mathrm{MHz}, \mathrm{CDCl}_{3}\right) \delta 162.8,142.2,141.5,140.5,129.5,129.1,128.8,128.5,128.2$, 128.0, 112.4, 110.0, 63.0, 13.7.

${ }^{19} \mathrm{~F}$ NMR $\left(376 \mathrm{MHz}, \mathrm{CDCl}_{3}\right) \delta-90.32(E),-95.55(Z)$.

HRMS (EI) calcd. for $\mathrm{C}_{18} \mathrm{H}_{15} \mathrm{~F}_{2} \mathrm{IO}_{2}{ }^{+}\left(\mathrm{M}^{+}\right) 428.0079$, found 428.0089

\section{ethyl $(E)$-2,2-difluoro-4-iodo-4-phenylbut-3-enoate ${ }^{3}$}<smiles>CCOC(=O)C(F)(F)/C=C(\I)c1ccccc1</smiles>

2j

Following general procedure $\mathbf{A}, \mathrm{Cs}_{2} \mathrm{CO}_{3}(\mathrm{MW}=325.8,0.4 \mathrm{mmol}, 130.3 \mathrm{mg}), \mathbf{1 j}(\mathrm{MW}=$ 102.0, $0.2 \mathrm{mmol}, 20.4 \mathrm{mg}$ ) and $\mathrm{ICF}_{2} \mathrm{COOEt}(\mathrm{MW}=250.0,0.4 \mathrm{mmol}, 100.0 \mathrm{mg}$ ) were added to $\mathrm{CH}_{3} \mathrm{CN}(1 \mathrm{~mL})$, stirred for $10 \mathrm{~h}$ under $30 \mathrm{~W}$ blue LED irradiation at room temperature, the reaction mixture was purified by silica gel chromatography $(\mathrm{PE} / \mathrm{EA}=60: 1)$ to obtain product $\mathbf{2 j}$ as colorless liquid (MW $=352.0,63.1 \mathrm{mg}, 0.179 \mathrm{mmol}, 90 \%$ yield, $E / Z=85: 15$ ).

${ }^{1} \mathrm{H}$ NMR (400 MHz, $\left.\mathrm{CDCl}_{3}\right) \delta 7.39-7.26(\mathrm{~m}, 5 \mathrm{H}), 6.72(\mathrm{t}, J=11.0 \mathrm{~Hz}, 1 \mathrm{H}), 3.96(\mathrm{q}, J=7.2$ $\mathrm{Hz}, 2 \mathrm{H}), 1.19(\mathrm{t}, J=7.2 \mathrm{~Hz}, 3 \mathrm{H})$.

${ }^{13} \mathrm{C}$ NMR $\left(101 \mathrm{MHz}, \mathrm{CDCl}_{3}\right) \delta 162.9,162.6,162.2,140.7,133.3,133.1,132.8,129.5,128.1$, 127.8, 113.4, 110.9, 108.7, 108.4, 63.2, 13.7.

${ }^{19} \mathrm{~F}$ NMR $\left(376 \mathrm{MHz}, \mathrm{CDCl}_{3}\right) \delta-93.80(E),-98.06(Z)$. 


\section{ethyl $(E)-2,2-d i f l u o r o-4-i o d o-4-\left(p\right.$-tolyl)but-3-enoate ${ }^{3}$}<smiles>CCOC(=O)CC(F)=CC(I)c1ccc(C)cc1</smiles>

2k

Following general procedure $\mathbf{A}, \mathrm{Cs}_{2} \mathrm{CO}_{3}(\mathrm{MW}=325.8,0.4 \mathrm{mmol}, 130.3 \mathrm{mg}), \mathbf{1 k}(\mathrm{MW}=$ $116.1,0.2 \mathrm{mmol}, 23.2 \mathrm{mg}$ ) and $\mathrm{ICF}_{2} \mathrm{COOEt}(\mathrm{MW}=250.0,0.4 \mathrm{mmol}, 100.0 \mathrm{mg}$ ) were added to $\mathrm{CH}_{3} \mathrm{CN}(1 \mathrm{~mL})$, stirred for $15 \mathrm{~h}$ under $30 \mathrm{~W}$ blue LED irradiation at room temperature, the reaction mixture was purified by silica gel chromatography $(\mathrm{PE} / \mathrm{EA}=60: 1)$ to obtain product 2k as colorless liquid ( $\mathrm{MW}=366.0,54.9 \mathrm{mg}, 0.150 \mathrm{mmol}, 75 \%$ yield, $E / Z=85: 15$ ).

${ }^{1} \mathrm{H}$ NMR $\left(400 \mathrm{MHz}, \mathrm{CDCl}_{3}\right) \delta 7.19(\mathrm{~d}, J=8.1 \mathrm{~Hz}, 2 \mathrm{H}), 7.11(\mathrm{~d}, J=8.0 \mathrm{~Hz}, 2 \mathrm{H}), 6.68(\mathrm{t}, J=$ $10.9 \mathrm{~Hz}, 1 \mathrm{H}), 3.97$ (q, $J=7.1 \mathrm{~Hz}, 2 \mathrm{H}), 2.33(\mathrm{~s}, 3 \mathrm{H}), 1.19$ (t, $J=7.2 \mathrm{~Hz}, 3 \mathrm{H})$.

${ }^{13} \mathrm{C}$ NMR $\left(101 \mathrm{MHz}, \mathrm{CDCl}_{3}\right) \delta 163.0,162.6,162.3,139.7,137.9,133.0,132.7,132.5,129.2$, 128.7, 127.9, 113.4, 110.9, 109.3, 63.1, 21.4, 13.7.

${ }^{19} \mathrm{~F}$ NMR $\left(376 \mathrm{MHz}, \mathrm{CDCl}_{3}\right) \delta-93.66(E),-97.81(Z)$.

ethyl $(E)$-2,2-difluoro-4-iodo-4-(m-tolyl)but-3-enoate ${ }^{4}$<smiles>CCOC(=O)C(F)(F)C=C(I)c1cccc(C)c1</smiles>

2I

Following general procedure $\mathbf{A}, \mathrm{Cs}_{2} \mathrm{CO}_{3}(\mathrm{MW}=325.8,0.4 \mathrm{mmol}, 130.3 \mathrm{mg}), \mathbf{1 l}(\mathrm{MW}=116.1$, $0.2 \mathrm{mmol}, 23.2 \mathrm{mg}$ ) and $\mathrm{ICF}_{2} \mathrm{COOEt}(\mathrm{MW}=250.0,0.4 \mathrm{mmol}, 100.0 \mathrm{mg}$ ) were added to $\mathrm{CH}_{3} \mathrm{CN}(1 \mathrm{~mL})$, stirred for $15 \mathrm{~h}$ under $30 \mathrm{~W}$ blue LED irradiation at room temperature, the reaction mixture was purified by silica gel chromatography $(\mathrm{PE} / \mathrm{EA}=60: 1)$ to obtain product $2 \mathbf{l}$ as colorless liquid (MW $=366.0,55.6 \mathrm{mg}, 0.152 \mathrm{mmol}, 76 \%$ yield, $E / \mathrm{Z}=89: 11$ ).

${ }^{1} \mathrm{H}$ NMR (400 MHz, $\left.\mathrm{CDCl}_{3}\right) \delta 7.24-7.05(\mathrm{~m}, 4 \mathrm{H}), 6.69(\mathrm{t}, J=10.8 \mathrm{~Hz}, 1 \mathrm{H}), 3.95(\mathrm{q}, J=7.2$ $\mathrm{Hz}, 2 \mathrm{H}), 2.33$ (s, 3H), 1.19 (t, $J=7.2 \mathrm{~Hz}, 3 \mathrm{H})$.

${ }^{13} \mathrm{C}$ NMR $\left(101 \mathrm{MHz}, \mathrm{CDCl}_{3}\right) \delta 162.9,162.5,162.2,140.6,137.8,133.1,132.9,132.6,130.3$, 128.4, 128.0, 125.0, 113.4, 110.9, 109.0, 108.4, 63.1, 21.3, 13.7.

${ }^{19} \mathrm{~F}$ NMR $\left(376 \mathrm{MHz}, \mathrm{CDCl}_{3}\right) \delta-93.65(E),-98.00(Z)$.

ethyl (E)-2,2-difluoro-4-iodo-4-(o-tolyl)but-3-enoate ${ }^{4}$

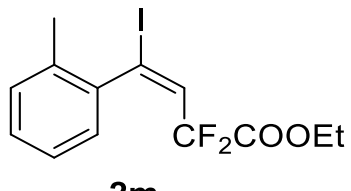

Following general procedure $\mathbf{A}, \mathrm{Cs}_{2} \mathrm{CO}_{3}(\mathrm{MW}=325.8,0.4 \mathrm{mmol}, 130.3 \mathrm{mg}), \mathbf{1 m}(\mathrm{MW}=$ $116.1,0.2 \mathrm{mmol}, 23.2 \mathrm{mg}$ ) and $\mathrm{ICF}_{2} \mathrm{COOEt}(\mathrm{MW}=250.0,0.4 \mathrm{mmol}, 100.0 \mathrm{mg}$ ) were added to $\mathrm{CH}_{3} \mathrm{CN}(1 \mathrm{~mL})$, stirred for $12 \mathrm{~h}$ under $30 \mathrm{~W}$ blue LED irradiation at room temperature, the reaction mixture was purified by silica gel chromatography $(\mathrm{PE} / \mathrm{EA}=60: 1)$ to obtain product 
$\mathbf{2 m}$ as colorless liquid (MW $=366.0,59.1 \mathrm{mg}, 0.162 \mathrm{mmol}, 81 \%$ yield, $E / Z=97: 3$ ).

${ }^{1} \mathrm{H}$ NMR (400 MHz, $\left.\mathrm{CDCl}_{3}\right) \delta 7.25-7.06(\mathrm{~m}, 4 \mathrm{H}), 6.75(\mathrm{dd}, J=11.1,10.1 \mathrm{~Hz}, 1 \mathrm{H}), 3.98(\mathrm{q}$, $J=7.1 \mathrm{~Hz}, 2 \mathrm{H}), 2.27(\mathrm{~s}, 3 \mathrm{H}), 1.22(\mathrm{t}, J=7.2 \mathrm{~Hz}, 3 \mathrm{H})$.

${ }^{13} \mathrm{C}$ NMR $\left(101 \mathrm{MHz}, \mathrm{CDCl}_{3}\right) \delta 162.9,162.5,139.7,134.8,134.0,133.8,133.5,130.4,129.4$, 127.2, 125.5, 113.3, 110.8, 108.3, 107.9, 63.2, 19.5, 13.7.

${ }^{19} \mathrm{~F}$ NMR $\left(376 \mathrm{MHz}, \mathrm{CDCl}_{3}\right) \delta-95.45,-96.17,-97.43,-98.15$.

ethyl (E)-2,2-difluoro-4-iodo-4-(4-methoxyphenyl)but-3-enoate ${ }^{3}$<smiles>CCOC(=O)C(F)(F)/C=C(/I)c1ccc(OC)cc1</smiles>

$2 n$

Following general procedure $\mathbf{A}, \mathrm{Cs}_{2} \mathrm{CO}_{3}(\mathrm{MW}=325.8,0.4 \mathrm{mmol}, 130.3 \mathrm{mg}), \mathbf{1 n}(\mathrm{MW}=$ $132.1,0.2 \mathrm{mmol}, 26.4 \mathrm{mg})$ and $\mathrm{ICF}_{2} \mathrm{COOEt}(\mathrm{MW}=250.0,0.4 \mathrm{mmol}, 100.0 \mathrm{mg})$ were added to $\mathrm{CH}_{3} \mathrm{CN}(1 \mathrm{~mL})$, stirred for $12 \mathrm{~h}$ under $30 \mathrm{~W}$ blue LED irradiation at room temperature, the reaction mixture was purified by silica gel chromatography $(\mathrm{PE} / \mathrm{EA}=30: 1)$ to obtain product 2n as faint yellow liquid ( $\mathrm{MW}=382.0,66.6 \mathrm{mg}, 0.174 \mathrm{mmol}, 87 \%$ yield, $E / Z=78: 22$ ).

${ }^{1} \mathrm{H}$ NMR $\left(400 \mathrm{MHz}, \mathrm{CDCl}_{3}\right) \delta 7.26(\mathrm{~d}, J=8.8 \mathrm{~Hz}, 2 \mathrm{H}), 6.82(\mathrm{~d}, J=8.8 \mathrm{~Hz}, 2 \mathrm{H}), 6.67(\mathrm{t}, J=$ $10.8 \mathrm{~Hz}, 1 \mathrm{H}), 3.98$ (q, $J=7.2 \mathrm{~Hz}, 2 \mathrm{H}), 3.80(\mathrm{~s}, 3 \mathrm{H}), 1.19$ (t, $J=7.2 \mathrm{~Hz}, 3 \mathrm{H})$.

${ }^{13} \mathrm{C}$ NMR $\left(101 \mathrm{MHz}, \mathrm{CDCl}_{3}\right) \delta 162.9,162.6,162.3,160.4,132.9,132.5,132.2,129.8,128.7$, 128.4, 128.1, 110.9, 109.4, 63.1, 55.4, 13.7.

${ }^{19} \mathrm{~F}$ NMR $\left(376 \mathrm{MHz}, \mathrm{CDCl}_{3}\right) \delta-93.25(E),-97.52(Z)$.

\section{ethyl $(E)$-2,2-difluoro-4-iodo-4-(3-methoxyphenyl)but-3-enoate ${ }^{3}$}<smiles>CCOC(=O)C(F)(F)/C=C(/I)c1cccc(OC)c1</smiles>

20

Following general procedure $\mathbf{A}, \mathrm{Cs}_{2} \mathrm{CO}_{3}(\mathrm{MW}=325.8,0.4 \mathrm{mmol}, 130.3 \mathrm{mg}), 1 \mathrm{o}(\mathrm{MW}=$ 132.1, $0.2 \mathrm{mmol}, 26.4 \mathrm{mg}$ ) and $\mathrm{ICF}_{2} \mathrm{COOEt}(\mathrm{MW}=250.0,0.4 \mathrm{mmol}, 100.0 \mathrm{mg}$ ) were added to $\mathrm{CH}_{3} \mathrm{CN}(1 \mathrm{~mL})$, stirred for $14 \mathrm{~h}$ under $30 \mathrm{~W}$ blue LED irradiation at room temperature, the reaction mixture was purified by silica gel chromatography $(\mathrm{PE} / \mathrm{EA}=30: 1)$ to obtain product $2 \mathrm{o}$ as colorless liquid (MW = 382.0, $54.4 \mathrm{mg}, 0.142 \mathrm{mmol}, 71 \%$ yield, $E / Z=96: 4$ ).

${ }^{1} \mathrm{H}$ NMR $\left(400 \mathrm{MHz}, \mathrm{CDCl}_{3}\right) \delta 7.22(\mathrm{t}, J=7.9 \mathrm{~Hz}, 1 \mathrm{H}), 6.89$ (d, $\left.J=7.6 \mathrm{~Hz}, 1 \mathrm{H}\right), 6.83$ (ddd, $J$ $=6.6,6.1,2.1 \mathrm{~Hz}, 2 \mathrm{H}), 6.70(\mathrm{t}, J=10.9 \mathrm{~Hz}, 1 \mathrm{H}), 3.99(\mathrm{q}, J=7.2 \mathrm{~Hz}, 2 \mathrm{H}), 3.81(\mathrm{~s}, 3 \mathrm{H}), 1.20$ (t, $J=7.2 \mathrm{~Hz}, 3 \mathrm{H})$.

${ }^{13} \mathrm{C}$ NMR $\left(101 \mathrm{MHz}, \mathrm{CDCl}_{3}\right) \delta 162.5,158.9,141.8,133.3,133.0,132.8,129.2,115.5,113.4$, 113.1, 110.9, 108.4, 63.1, 55.3, 13.7.

${ }^{19} \mathrm{~F} \mathrm{NMR}\left(376 \mathrm{MHz}, \mathrm{CDCl}_{3}\right) \delta-93.76(E),-98.10(Z)$.

ethyl (E)-2,2-difluoro-4-(4-fluorophenyl)-4-iodobut-3-enoate ${ }^{3}$ 
<smiles>CCOC(=O)C(F)(F)/C=C(/I)c1ccc(F)cc1</smiles>

Following general procedure $\mathbf{A}, \mathrm{Cs}_{2} \mathrm{CO}_{3}(\mathrm{MW}=325.8,0.4 \mathrm{mmol}, 130.3 \mathrm{mg}), \mathbf{1 p}(\mathrm{MW}=$ 120.0, $0.2 \mathrm{mmol}, 24.0 \mathrm{mg}$ ) and $\mathrm{ICF}_{2} \mathrm{COOEt}(\mathrm{MW}=250.0,0.4 \mathrm{mmol}, 100.0 \mathrm{mg}$ ) were added to $\mathrm{CH}_{3} \mathrm{CN}(1 \mathrm{~mL})$, stirred for $12 \mathrm{~h}$ under $30 \mathrm{~W}$ blue LED irradiation at room temperature, the reaction mixture was purified by silica gel chromatography $(\mathrm{PE} / \mathrm{EA}=60: 1)$ to obtain product 2p as faint yellow liquid (MW = 370.0, $57.1 \mathrm{mg}, 0.154 \mathrm{mmol}, 77 \%$ yield, $E / Z=96: 4$ ).

${ }^{1} \mathrm{H}$ NMR (400 MHz, $\left.\mathrm{CDCl}_{3}\right) \delta 7.34-7.27(\mathrm{~m}, 2 \mathrm{H}), 7.01(\mathrm{t}, J=8.6 \mathrm{~Hz}, 2 \mathrm{H}), 6.71(\mathrm{t}, J=11.2$ $\mathrm{Hz}, 1 \mathrm{H}), 4.05(\mathrm{q}, J=7.2 \mathrm{~Hz}, 2 \mathrm{H}), 1.23(\mathrm{t}, J=7.2 \mathrm{~Hz}, 3 \mathrm{H})$.

${ }^{13} \mathrm{C}$ NMR $\left(101 \mathrm{MHz}, \mathrm{CDCl}_{3}\right) \delta 164.1,162.9,162.5,162.2,161.7,136.9,133.7,133.4,133.1$, 129.9, 115.6, 115.3, 115.1, 113.4, 110.9, 108.4, 107.3, 63.3, 13.8 .

${ }^{19} \mathrm{~F}$ NMR (376 MHz, $\left.\mathrm{CDCl}_{3}\right) \delta$-94.35 (E), -98.16 (Z), -110.61 (E), -110.84 (Z).

\section{ethyl (E)-4-(4-chlorophenyl)-2,2-difluoro-4-iodobut-3-enoate ${ }^{3}$}<smiles>CCOC(=O)CCCCOC(=O)c1ccc(Cl)cc1</smiles>

Following general procedure $\mathbf{A}, \mathrm{Cs}_{2} \mathrm{CO}_{3}$ (MW = 325.8, $\left.0.4 \mathrm{mmol}, 130.3 \mathrm{mg}\right), \mathbf{1 q}(\mathrm{MW}=$ 136.0, $0.2 \mathrm{mmol}, 27.2 \mathrm{mg}$ ) and $\mathrm{ICF}_{2} \mathrm{COOEt}(\mathrm{MW}=250.0,0.4 \mathrm{mmol}, 100.0 \mathrm{mg}$ ) were added to $\mathrm{CH}_{3} \mathrm{CN}(1 \mathrm{~mL})$, stirred for $12 \mathrm{~h}$ under $30 \mathrm{~W}$ blue LED irradiation at room temperature, the reaction mixture was purified by silica gel chromatography $(\mathrm{PE} / \mathrm{EA}=60: 1)$ to obtain product $\mathbf{2 q}$ as yellow liquid ( $\mathrm{MW}=385.9,55.5 \mathrm{mg}, 0.144 \mathrm{mmol}, 72 \%$ yield, $E / Z=89: 11$ ).

${ }^{1} \mathrm{H}$ NMR (400 MHz, $\left.\mathrm{CDCl}_{3}\right) \delta 7.38-7.21(\mathrm{~m}, 4 \mathrm{H}), 6.71(\mathrm{t}, J=11.3 \mathrm{~Hz}, 1 \mathrm{H}), 4.07(\mathrm{q}, J=7.2$ $\mathrm{Hz}, 2 \mathrm{H}), 1.24(\mathrm{t}, J=7.2 \mathrm{~Hz}, 3 \mathrm{H})$.

${ }^{13} \mathrm{C}$ NMR $\left(101 \mathrm{MHz}, \mathrm{CDCl}_{3}\right) \delta 162.5,162.2,139.3,135.4,133.5,129.2,128.3,110.9,108.4$, 107.0, 63.4, 13.8 .

${ }^{19} \mathrm{~F}$ NMR $\left(376 \mathrm{MHz}, \mathrm{CDCl}_{3}\right) \delta-94.63(E),-98.26(Z)$.

\section{(E)-1-bromo-4-(3-(ethylperoxy)-3,3-difluoro-1-iodoprop-1-en-1-yl)benzene ${ }^{3}$}<smiles>CCOC(=O)CC=C(I)c1ccc(Br)cc1</smiles>

Following general procedure $\mathbf{A}, \mathrm{Cs}_{2} \mathrm{CO}_{3}(\mathrm{MW}=325.8,0.4 \mathrm{mmol}, 130.3 \mathrm{mg}), \mathbf{1 r}(\mathrm{MW}=$ 180.0, $0.2 \mathrm{mmol}, 36.0 \mathrm{mg}$ ) and $\mathrm{ICF}_{2} \mathrm{COOEt}(\mathrm{MW}=250.0,0.4 \mathrm{mmol}, 100.0 \mathrm{mg}$ ) were added to $\mathrm{CH}_{3} \mathrm{CN}(1 \mathrm{~mL})$, stirred for $14 \mathrm{~h}$ under $30 \mathrm{~W}$ blue LED irradiation at room temperature, the reaction mixture was purified by silica gel chromatography $(\mathrm{PE} / \mathrm{EA}=60: 1)$ to obtain product 2r as colorless liquid ( $\mathrm{MW}=429.9,65.1 \mathrm{mg}, 0.151 \mathrm{mmol}, 76 \%$ yield, $E / Z=87: 13$ ). 
${ }^{1} \mathrm{H}$ NMR (400 MHz, CDCl3) $\delta 7.46(\mathrm{~d}, \mathrm{~J}=8.2 \mathrm{~Hz}, 2 \mathrm{H}), 7.18(\mathrm{~d}, \mathrm{~J}=8.2 \mathrm{~Hz}, 2 \mathrm{H}), 6.72(\mathrm{t}, \mathrm{J}=$ $11.1 \mathrm{~Hz}, 1 \mathrm{H}), 4.07$ (q, J = 7.1 Hz, 2H), 1.24 (t, J = 7.0 Hz, 3H).

${ }^{13} \mathrm{C}$ NMR $(101 \mathrm{MHz}, \mathrm{CDCl} 3) \delta 162.6,139.7,133.7,133.4,133.1,131.7,131.3,129.3,123.7$, 110.9, 107.0, 63.4, 13.8.

${ }^{19} \mathrm{~F}$ NMR (376 MHz, CDCl3) $\delta-94.65(E),-98.27(Z)$.

ethyl (E)-2,2-difluoro-4-iodo-4-(4-(trifluoromethyl)phenyl)but-3-enoate ${ }^{3}$<smiles>CCOC(=O)CC(F)(F)F</smiles>

Following general procedure $\mathbf{A}, \mathrm{Cs}_{2} \mathrm{CO}_{3}(\mathrm{MW}=325.8,0.4 \mathrm{mmol}, 130.3 \mathrm{mg}), \mathbf{1 s}(\mathrm{MW}=$ 170.0, $0.2 \mathrm{mmol}, 34.0 \mathrm{mg}$ ) and $\mathrm{ICF}_{2} \mathrm{COOEt}(\mathrm{MW}=250.0,0.4 \mathrm{mmol}, 100.0 \mathrm{mg}$ ) were added to $\mathrm{CH}_{3} \mathrm{CN}(1 \mathrm{~mL})$, stirred for $12 \mathrm{~h}$ under $30 \mathrm{~W}$ blue LED irradiation at room temperature, the reaction mixture was purified by silica gel chromatography $(\mathrm{PE} / \mathrm{EA}=60: 1)$ to obtain product 2s as colorless liquid ( $\mathrm{MW}=420.0,62.3 \mathrm{mg}, 0.148 \mathrm{mmol}, 74 \%$ yield, $E / Z=78: 22$ ).

${ }^{1} \mathrm{H}$ NMR $\left(400 \mathrm{MHz}, \mathrm{CDCl}_{3}\right) \delta 7.59(\mathrm{~d}, J=8.2 \mathrm{~Hz}, 2 \mathrm{H}), 7.42(\mathrm{~d}, J=8.2 \mathrm{~Hz}, 2 \mathrm{H}), 6.77(\mathrm{t}, J=$ $11.5 \mathrm{~Hz}, 1 \mathrm{H}), 4.09$ (q, $J=7.2 \mathrm{~Hz}, 2 \mathrm{H}), 1.24(\mathrm{t}, J=7.2 \mathrm{~Hz}, 3 \mathrm{H})$.

${ }^{13} \mathrm{C}$ NMR $\left(101 \mathrm{MHz}, \mathrm{CDCl}_{3}\right) \delta 162.8,162.5,162.2,144.4,134.1,133.8,133.5,131.3,131.0$, 128.1, 125.1, 110.9, 106.0, 63.4, 13.7.

${ }^{19} \mathrm{~F}$ NMR (376 MHz, $\left.\mathrm{CDCl}_{3}\right) \delta-62.94(E),-63.20(Z),-95.28(E),-98.66(Z)$.

\section{(E)-4-(3-(ethylperoxy)-3,3-difluoro-1-iodoprop-1-en-1-yl)benzonitrile ${ }^{3}$}<smiles>[Z12]C(=O)OCC</smiles>

Following general procedure $\mathbf{A}, \mathrm{Cs}_{2} \mathrm{CO}_{3}(\mathrm{MW}=325.8,0.4 \mathrm{mmol}, 130.3 \mathrm{mg}), \mathbf{1 t}(\mathrm{MW}=$ 127.0, $0.2 \mathrm{mmol}, 25.4 \mathrm{mg}$ ) and $\mathrm{ICF}_{2} \mathrm{COOEt}(\mathrm{MW}=250.0,0.4 \mathrm{mmol}, 100.0 \mathrm{mg}$ ) were added to $\mathrm{CH}_{3} \mathrm{CN}(1 \mathrm{~mL})$, stirred for $14 \mathrm{~h}$ under $30 \mathrm{~W}$ blue LED irradiation at room temperature, the reaction mixture was purified by silica gel chromatography $(\mathrm{PE} / \mathrm{EA}=25: 1)$ to obtain product $2 t$ as colorless liquid ( $\mathrm{MW}=377.0,40.8 \mathrm{mg}, 0.108 \mathrm{mmol}, 54 \%$ yield, $E / \mathrm{Z}=77: 23$ ).

${ }^{1} \mathrm{H}$ NMR $\left(400 \mathrm{MHz}, \mathrm{CDCl}_{3}\right) \delta 7.64(\mathrm{~d}, J=8.6 \mathrm{~Hz}, 2 \mathrm{H}), 7.40(\mathrm{~d}, J=8.4 \mathrm{~Hz}, 2 \mathrm{H}), 6.76(\mathrm{t}, J=$ $11.8 \mathrm{~Hz}, 1 \mathrm{H}), 4.17$ (q, $J=7.2 \mathrm{~Hz}, 2 \mathrm{H}), 1.29$ (t, $J=7.2 \mathrm{~Hz}, 3 \mathrm{H})$.

${ }^{13} \mathrm{C}$ NMR $\left(101 \mathrm{MHz}, \mathrm{CDCl}_{3}\right) \delta 162.4,162.1,145.3,134.1,133.8,133.6,131.9,128.3,118.1$, 113.0, 110.9, 105.2, 63.6, 13.9 .

${ }^{19} \mathrm{~F} \mathrm{NMR}\left(376 \mathrm{MHz}, \mathrm{CDCl}_{3}\right) \delta-95.96(E)$.

ethyl $(E)-2,2-d i f l u o r o-4-i o d o-4-(p y r i d i n-2-y l) b u t-3-e n o a t e$ 


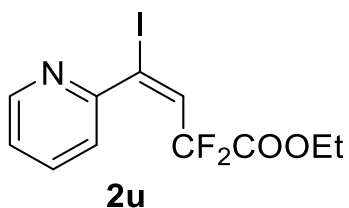

Following general procedure $\mathbf{A}, \mathrm{Cs}_{2} \mathrm{CO}_{3}(\mathrm{MW}=325.8,0.4 \mathrm{mmol}, 130.3 \mathrm{mg}), \mathbf{1 u}(\mathrm{MW}=$ 103.0, $0.2 \mathrm{mmol}, 20.6 \mathrm{mg})$ and $\mathrm{ICF}_{2} \mathrm{COOEt}(\mathrm{MW}=250.0,0.4 \mathrm{mmol}, 100.0 \mathrm{mg})$ were added to $\mathrm{CH}_{3} \mathrm{CN}(1 \mathrm{~mL})$, stirred for $12 \mathrm{~h}$ under $30 \mathrm{~W}$ blue LED irradiation at room temperature, the reaction mixture was purified by silica gel chromatography $(\mathrm{PE} / \mathrm{EA}=40: 1)$ to obtain product $\mathbf{2 u}$ as yellow liquid ( $\mathrm{MW}=353.0,46.1 \mathrm{mg}, 0.130 \mathrm{mmol}, 65 \%$ yield, $E / Z=78: 22$ ).

${ }^{1} \mathrm{H}$ NMR $\left(400 \mathrm{MHz}, \mathrm{CDCl}_{3}\right) \delta 8.48(\mathrm{ddd}, J=4.8,1.8,0.9 \mathrm{~Hz}, 1 \mathrm{H}), 7.81-7.67(\mathrm{~m}, 2 \mathrm{H}), 7.59$ $(\mathrm{dt}, J=7.9,1.0 \mathrm{~Hz}, 1 \mathrm{H}), 6.87(\mathrm{t}, J=12.2 \mathrm{~Hz}, 1 \mathrm{H}), 4.21(\mathrm{q}, J=7.2 \mathrm{~Hz}, 2 \mathrm{H}), 1.27(\mathrm{t}, J=7.2$ $\mathrm{Hz}, 3 \mathrm{H})$.

${ }^{13} \mathrm{C}$ NMR $\left(101 \mathrm{MHz}, \mathrm{CDCl}_{3}\right) \delta 162.6,155.5,149.3,147.9,137.2,137.0,135.9,135.6,135.3$, 132.7, 132.4, 124.6, 124.3, 123.8, 123.4, 113.9, 112.6, 111.4, 107.1, 63.5, 62.8, 13.9.

${ }^{19} \mathrm{~F} \mathrm{NMR}\left(376 \mathrm{MHz}, \mathrm{CDCl}_{3}\right) \delta-95.20(E),-98.16(Z)$.

HRMS (EI) calcd. for $\mathrm{C}_{11} \mathrm{H}_{10} \mathrm{~F}_{2} \mathrm{INO}_{2}{ }^{+}\left(\mathrm{M}^{+}\right) 352.9719$, found 352.9729 .

ethyl $(E)$-2,2-difluoro-4-iodododec-3-enoate ${ }^{5}$

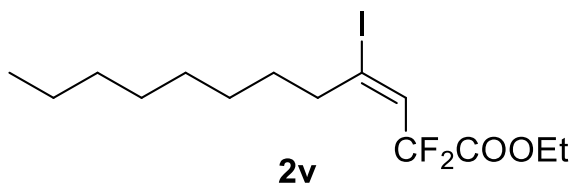

Following general procedure $\mathbf{A}, \mathrm{Cs}_{2} \mathrm{CO}_{3}(\mathrm{MW}=325.8,0.4 \mathrm{mmol}, 130.3 \mathrm{mg}), \mathbf{1 v}(\mathrm{MW}=$ 138.1, $0.2 \mathrm{mmol}, 27.6 \mathrm{mg})$ and $\mathrm{ICF}_{2} \mathrm{COOEt}(\mathrm{MW}=250.0,0.4 \mathrm{mmol}, 100.0 \mathrm{mg})$ were added to $\mathrm{CH}_{3} \mathrm{CN}(1 \mathrm{~mL})$, stirred for $12 \mathrm{~h}$ under $30 \mathrm{~W}$ blue LED irradiation at room temperature, the reaction mixture was purified by silica gel chromatography $(\mathrm{PE} / \mathrm{EA}=60: 1)$ to obtain product $2 \mathrm{v}$ as colorless liquid (MW $=388.1,59.1 \mathrm{mg}, 0.152 \mathrm{mmol}, 76 \%$ yield, $E / Z=83: 17$ ).

${ }^{1} \mathrm{H}$ NMR $\left(400 \mathrm{MHz}, \mathrm{CDCl}_{3}\right) \delta 6.40(\mathrm{t}, J=13.2 \mathrm{~Hz}, 1 \mathrm{H}), 4.33(\mathrm{q}, J=7.2 \mathrm{~Hz}, 2 \mathrm{H}), 2.60(\mathrm{dd}, J$ $=11.0,3.9 \mathrm{~Hz}, 2 \mathrm{H}), 1.31(\mathrm{ddd}, J=13.5,11.9,4.0 \mathrm{~Hz}, 15 \mathrm{H}), 0.88(\mathrm{t}, J=6.9 \mathrm{~Hz}, 3 \mathrm{H})$.

${ }^{13} \mathrm{C}$ NMR $\left(101 \mathrm{MHz}, \mathrm{CDCl}_{3}\right) \delta 163.6,163.3,162.9,131.5,131.2,130.9,119.7,111.6,63.3$, 46.8, 40.8, 31.8, 29.9, 29.2, 28.4, 22.7, 14.0.

${ }^{19} \mathrm{~F}$ NMR $\left(376 \mathrm{MHz}, \mathrm{CDCl}_{3}\right) \delta-89.09(Z),-97.71(E)$.

\section{Characterization data of hydrodifluoroalkylation products}

\section{ethyl 2,2-difluoro-3-methyl-4-phenylbut-3-enoate ${ }^{6}$}

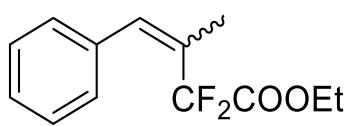

3a

Following general procedure $\mathbf{A}, \mathrm{Cs}_{2} \mathrm{CO}_{3}(\mathrm{MW}=325.8,0.4 \mathrm{mmol}, 130.3 \mathrm{mg}), \mathbf{1 a}(\mathrm{MW}=$ $116.1,0.2 \mathrm{mmol}, 23.2 \mathrm{mg})$ and $\mathrm{ICF}_{2} \mathrm{COOEt}(\mathrm{MW}=250.0,0.4 \mathrm{mmol}, 100.0 \mathrm{mg})$ were added to THF $(1 \mathrm{~mL})$, stirred for $60 \mathrm{~h}$ under $30 \mathrm{~W}$ blue LED irradiation at room temperature, the reaction mixture was purified by silica gel chromatography $(\mathrm{PE} / \mathrm{EA}=60: 1)$ to obtain product 
3a as colorless liquid (MW $=240.1,38.9 \mathrm{mg}, 0.162 \mathrm{mmol}, 81 \%$ yield, $E / Z=54: 46$ ).

${ }^{1} \mathrm{H}$ NMR $\left(400 \mathrm{MHz}, \mathrm{CDCl}_{3}\right) \delta 7.41-7.19(\mathrm{~m}, 10 \mathrm{H}, Z+E), 6.95(\mathrm{~s}, 1 \mathrm{H}, E), 6.81(\mathrm{~s}, 1 \mathrm{H}, Z)$, $4.36(\mathrm{q}, J=7.1 \mathrm{~Hz}, 2 \mathrm{H}, E), 3.88(\mathrm{q}, J=7.2 \mathrm{~Hz}, 2 \mathrm{H}, Z), 2.06(\mathrm{~d}, J=1.5 \mathrm{~Hz}, 3 \mathrm{H}, Z), 1.99$ (d, $J$ $=1.4 \mathrm{~Hz}, 3 \mathrm{H}, E), 1.36(\mathrm{t}, J=7.1 \mathrm{~Hz}, 3 \mathrm{H}, E), 1.11(\mathrm{t}, J=7.2 \mathrm{~Hz}, 3 \mathrm{H}, Z)$.

${ }^{13} \mathrm{C}$ NMR $\left(101 \mathrm{MHz}, \mathrm{CDCl}_{3}\right) \delta 163.9,163.4,135.2,134.3,131.0,130.0,129.2,129.0,128.7$, 128.4, 127.9, 116.9, 114.4, 113.3, 111.9, 110.83 63.0, 62.7, 19.2, 14.0, 13.6, 12.6.

${ }^{19} \mathrm{~F}$ NMR $\left(376 \mathrm{MHz}, \mathrm{CDCl}_{3}\right) \delta-97.17(Z),-106.62(E)$.

ethyl 2,2-difluoro-3-methyl-4-(p-tolyl)but-3-enoate<smiles>CCOC(=O)C(C)=C(C)C=Cc1ccc(C)cc1</smiles>

$3 \mathbf{b}$

Following general procedure $\mathbf{A}, \mathrm{Cs}_{2} \mathrm{CO}_{3}(\mathrm{MW}=325.8,0.4 \mathrm{mmol}, 130.3 \mathrm{mg}), \mathbf{1 b}(\mathrm{MW}=$ $130.1,0.2 \mathrm{mmol}, 26.0 \mathrm{mg}$ ) and $\mathrm{ICF}_{2} \mathrm{COOEt}(\mathrm{MW}=250.0,0.4 \mathrm{mmol}, 100.0 \mathrm{mg}$ ) were added to THF $(1 \mathrm{~mL})$, stirred for $60 \mathrm{~h}$ under $30 \mathrm{~W}$ blue LED irradiation at room temperature, the reaction mixture was purified by silica gel chromatography $(\mathrm{PE} / \mathrm{EA}=60: 1)$ to obtain product 3b as colorless liquid ( $\mathrm{MW}=254.1,40.5 \mathrm{mg}, 0.159 \mathrm{mmol}, 80 \%$ yield, $E / Z=56: 44$ ).

${ }^{1} \mathrm{H}$ NMR (400 MHz, $\left.\mathrm{CDCl}_{3}\right) \delta 7.20(\mathrm{q}, J=8.2 \mathrm{~Hz}, 4 \mathrm{H}, E), 7.11(\mathrm{~s}, 4 \mathrm{H}, Z), 6.91(\mathrm{~s}, 1 \mathrm{H}, E)$, 6.77 (s, 1H, Z), 4.35 (q, $J=7.1 \mathrm{~Hz}, 2 \mathrm{H}, E), 3.89$ (q, $J=7.2 \mathrm{~Hz}, 2 \mathrm{H}, Z), 2.36$ (s, 3H, E), 2.32 (s, $3 \mathrm{H}, Z), 2.05$ (d, $J=1.4 \mathrm{~Hz}, 3 \mathrm{H}, Z), 1.99$ (d, $J=1.4 \mathrm{~Hz}, 3 \mathrm{H}, E), 1.35$ (t, $J=7.1 \mathrm{~Hz}, 3 \mathrm{H}, E)$, $1.11(\mathrm{t}, J=7.2 \mathrm{~Hz}, 3 \mathrm{H}, Z)$.

${ }^{13} \mathrm{C}$ NMR $\left(101 \mathrm{MHz}, \mathrm{CDCl}_{3}\right) \delta 164.5,164.1,163.8,163.5,138.0,137.6,134.4,132.3,130.9$, 129.2, $128.9-128.6,114.6,113.4,63.0,62.8,21.3,19.2,14.0,13.6,12.6$.

${ }^{19} \mathrm{~F}$ NMR $\left(376 \mathrm{MHz}, \mathrm{CDCl}_{3}\right) \delta-97.02(Z),-106.50(E)$.

HRMS (EI) calcd. for $\mathrm{C}_{14} \mathrm{H}_{16} \mathrm{~F}_{2} \mathrm{O}_{2}{ }^{+}\left(\mathrm{M}^{+}\right)$254.1113, found 254.1125.

ethyl 2,2-difluoro-4-(4-methoxyphenyl)-3-methylbut-3-enoate<smiles>CCOC(=O)C(C)=Cc1ccc(OC)cc1</smiles>

Following general procedure $\mathbf{A}, \mathrm{Cs}_{2} \mathrm{CO}_{3}(\mathrm{MW}=325.8,0.4 \mathrm{mmol}, 130.3 \mathrm{mg}), \mathbf{1 c}(\mathrm{MW}=$ 146.1, $0.2 \mathrm{mmol}, 29.2 \mathrm{mg}$ ) and $\mathrm{ICF}_{2} \mathrm{COOEt}(\mathrm{MW}=250.0,0.4 \mathrm{mmol}, 100.0 \mathrm{mg}$ ) were added to THF ( $1 \mathrm{~mL})$, stirred for $60 \mathrm{~h}$ under $30 \mathrm{~W}$ blue LED irradiation at room temperature, the reaction mixture was purified by silica gel chromatography $(\mathrm{PE} / \mathrm{EA}=30: 1)$ to obtain product $3 c$ as colorless liquid ( $\mathrm{MW}=270.1,42.1 \mathrm{mg}, 0.156 \mathrm{mmol}, 78 \%$ yield, $E / Z=58: 42$ ).

${ }^{1} \mathrm{H}$ NMR (400 MHz, $\left.\mathrm{CDCl}_{3}\right) \delta 7.29(\mathrm{~d}, J=8.7 \mathrm{~Hz}, 2 \mathrm{H}, E), 7.17$ (d, $\left.J=8.6 \mathrm{~Hz}, 2 \mathrm{H}, Z\right), 6.91(\mathrm{~d}$, $J=8.8 \mathrm{~Hz}, 2 \mathrm{H}, E), 6.87(\mathrm{~s}, 1 \mathrm{H}, E), 6.83(\mathrm{~d}, J=8.8 \mathrm{~Hz}, 2 \mathrm{H}, Z), 6.74(\mathrm{~s}, 1 \mathrm{H}, Z), 4.35$ (q, $J=$ $7.1 \mathrm{~Hz}, 2 \mathrm{H}, E), 3.92(\mathrm{q}, J=7.2 \mathrm{~Hz}, 2 \mathrm{H}, Z), 3.82(\mathrm{~s}, 3 \mathrm{H}, E), 3.79$ (s, 3H, Z), 2.04 (d, $J=1.4 \mathrm{~Hz}$, $3 \mathrm{H}, Z), 1.99(\mathrm{~d}, J=1.3 \mathrm{~Hz}, 3 \mathrm{H}, E), 1.36(\mathrm{t}, J=7.1 \mathrm{~Hz}, 3 \mathrm{H}, E), 1.12$ (t, $J=7.2 \mathrm{~Hz}, 3 \mathrm{H}, Z$ ).

${ }^{13} \mathrm{C}$ NMR $\left(101 \mathrm{MHz}, \mathrm{CDCl}_{3}\right) \delta 164.2,163.8,163.6,163.2,159.2,134.1,131.0-130.1,128.7$, 127.6, 126.8, 114.6, 113.8, 113.7 - 113.1, 112.1, 63.0, 62.8, 55.3, 19.2, 14.0, 13.6, 12.6.

${ }^{19} \mathrm{~F}$ NMR (376 MHz, $\left.\mathrm{CDCl}_{3}\right) \delta-96.84(Z),-106.32(E)$. 
HRMS (EI) calcd. for $\mathrm{C}_{14} \mathrm{H}_{16} \mathrm{~F}_{2} \mathrm{O}_{3}{ }^{+}\left(\mathrm{M}^{+}\right)$270.1062, found 270.1065 .

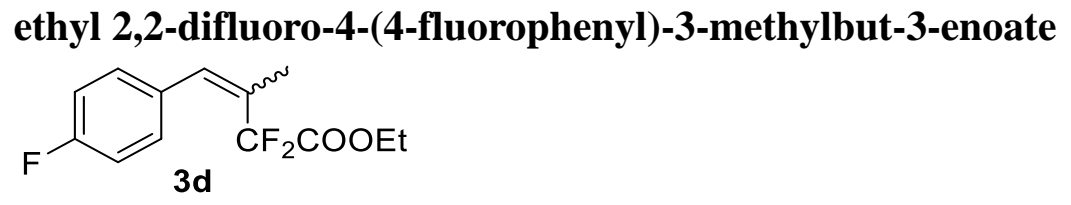

Following general procedure $\mathbf{A}, \mathrm{Cs}_{2} \mathrm{CO}_{3}(\mathrm{MW}=325.8,0.4 \mathrm{mmol}, 130.3 \mathrm{mg}), \mathbf{1 d}(\mathrm{MW}=$ 134.1, $0.2 \mathrm{mmol}, 26.8 \mathrm{mg}$ ) and $\mathrm{ICF}_{2} \mathrm{COOEt}(\mathrm{MW}=250.0,0.4 \mathrm{mmol}, 100.0 \mathrm{mg}$ ) were added to THF $(1 \mathrm{~mL})$, stirred for $60 \mathrm{~h}$ under $30 \mathrm{~W}$ blue LED irradiation at room temperature, the reaction mixture was purified by silica gel chromatography $(\mathrm{PE} / \mathrm{EA}=60: 1)$ to obtain product 3d as colorless liquid ( $\mathrm{MW}=258.1,42.8 \mathrm{mg}, 0.166 \mathrm{mmol}, 83 \%$ yield, $E / Z=55: 45$ ).

${ }^{1} \mathrm{H}$ NMR $\left(400 \mathrm{MHz}, \mathrm{CDCl}_{3}\right) \delta 7.30(\mathrm{dd}, J=8.6,5.5 \mathrm{~Hz}, 2 \mathrm{H}, E), 7.21(\mathrm{dd}, J=8.5,5.5 \mathrm{~Hz}, 2 \mathrm{H}$, Z), $7.07(\mathrm{t}, J=8.7 \mathrm{~Hz}, 2 \mathrm{H}, E), 6.99(\mathrm{t}, J=8.7 \mathrm{~Hz}, 2 \mathrm{H}, Z), 6.91(\mathrm{~s}, 1 \mathrm{H}, E), 6.75(\mathrm{~s}, 1 \mathrm{H}, Z), 4.36$ (q, $J=7.1 \mathrm{~Hz}, 2 \mathrm{H}, E), 3.97(\mathrm{q}, J=7.2 \mathrm{~Hz}, 2 \mathrm{H}, Z), 2.05(\mathrm{~d}, J=1.2 \mathrm{~Hz}, 3 \mathrm{H}, Z), 1.97$ (d, $J=1.3$ $\mathrm{Hz}, 3 \mathrm{H}, E), 1.36(\mathrm{t}, J=7.1 \mathrm{~Hz}, 3 \mathrm{H}, E), 1.16(\mathrm{t}, J=7.2 \mathrm{~Hz}, 3 \mathrm{H}, Z)$.

${ }^{13} \mathrm{C}$ NMR $\left(101 \mathrm{MHz}, \mathrm{CDCl}_{3}\right) \delta 164.0,163.5,161.0,133.2,131.1,130.6,129.9,116.8,115.8$, 115.6, 115.3, 115.0, 114.8, 114.3, 113.3, 111.8, 63.1, 62.9, 19.2, 14.0, 13.7, 12.5.

${ }^{19} \mathrm{~F}$ NMR (376 MHz, $\left.\mathrm{CDCl}_{3}\right) \delta-97.59(Z),-106.74(E),-113.16(Z),-113.82(E)$.

HRMS (EI) calcd. for $\mathrm{C}_{13} \mathrm{H}_{13} \mathrm{~F}_{3} \mathrm{O}_{2}{ }^{+}\left(\mathrm{M}^{+}\right)$258.0862, found 258.0862 .

ethyl 4-(4-chlorophenyl)-2,2-difluoro-3-methylbut-3-enoate<smiles>CCOC(=O)C(C)=Cc1ccc(Cl)cc1</smiles>

Following general procedure $\mathbf{A}, \mathrm{Cs}_{2} \mathrm{CO}_{3}(\mathrm{MW}=325.8,0.4 \mathrm{mmol}, 130.3 \mathrm{mg})$, 1e $(\mathrm{MW}=$ 150.0, $0.2 \mathrm{mmol}, 30.0 \mathrm{mg}$ ) and $\mathrm{ICF}_{2} \mathrm{COOEt}(\mathrm{MW}=250.0,0.4 \mathrm{mmol}, 100.0 \mathrm{mg}$ ) were added to THF (1mL), stirred for $60 \mathrm{~h}$ under $30 \mathrm{~W}$ blue LED irradiation at room temperature, the reaction mixture was purified by silica gel chromatography $(\mathrm{PE} / \mathrm{EA}=60: 1)$ to obtain product 3e as colorless liquid ( $\mathrm{MW}=274.1,46.7 \mathrm{mg}, 0.170 \mathrm{mmol}, 85 \%$ yield, $E / Z=52: 48$ ).

${ }^{1} \mathrm{H}$ NMR (400 MHz, CDCl $) \delta 7.35$ (d, $\left.J=8.5 \mathrm{~Hz}, 2 \mathrm{H}, E\right), 7.26$ (t, $J=8.5 \mathrm{~Hz}, 4 \mathrm{H}, Z$ ), 7.17 (d, $J=8.4 \mathrm{~Hz}, 2 \mathrm{H}, E), 6.90(\mathrm{~s}, 1 \mathrm{H}, E), 6.73(\mathrm{~s}, 1 \mathrm{H}, Z), 4.36(\mathrm{q}, J=7.1 \mathrm{~Hz}, 2 \mathrm{H}, E), 3.99$ (q, $J=$ $7.2 \mathrm{~Hz}, 2 \mathrm{H}, Z), 2.04(\mathrm{~d}, J=1.4 \mathrm{~Hz}, 3 \mathrm{H}, Z), 1.97(\mathrm{~d}, J=1.4 \mathrm{~Hz}, 3 \mathrm{H}, E), 1.36$ (t, $J=7.1 \mathrm{~Hz}, 3 \mathrm{H}$, $E), 1.17(\mathrm{t}, J=7.2 \mathrm{~Hz}, 3 \mathrm{H}, Z)$.

${ }^{13} \mathrm{C}$ NMR $\left(101 \mathrm{MHz}, \mathrm{CDCl}_{3}\right) \delta 164.0-163.3,163.1,134.0-133.5,133.1,130.6,130.1$, 129.8, 128.6, 128.1, 116.7, 115.8, 114.2, 113.3, 111.7, 110.8, 63.1, 19.3, 14.0, 13.7, 12.6.

${ }^{19} \mathrm{~F}$ NMR (376 MHz, $\left.\mathrm{CDCl}_{3}\right) \delta-97.89(Z),-106.84(E)$.

HRMS (EI) calcd. for $\mathrm{C}_{13} \mathrm{H}_{13} \mathrm{ClF}_{2} \mathrm{O}_{2}{ }^{+}\left(\mathrm{M}^{+}\right) 274.0567$, found 274.0578 .

\section{ethyl 3-benzylidene-2,2-difluoropentanoate}<smiles>CCOC(=O)C(=Cc1ccccc1)C(=Cc1ccccc1)C(=O)OCC</smiles>

Following general procedure $\mathbf{A}, \mathrm{Cs}_{2} \mathrm{CO}_{3}(\mathrm{MW}=325.8,0.4 \mathrm{mmol}, 130.3 \mathrm{mg})$, $\mathbf{1 f}(\mathrm{MW}=$ 
130.1, $0.2 \mathrm{mmol}, 26.0 \mathrm{mg}$ ) and $\mathrm{ICF}_{2} \mathrm{COOEt}(\mathrm{MW}=250.0,0.4 \mathrm{mmol}, 100.0 \mathrm{mg}$ ) were added to THF ( $1 \mathrm{~mL})$, stirred for $60 \mathrm{~h}$ under $30 \mathrm{~W}$ blue LED irradiation at room temperature, the reaction mixture was purified by silica gel chromatography $(\mathrm{PE} / \mathrm{EA}=60: 1)$ to obtain product 3f as colorless liquid ( $\mathrm{MW}=254.1,39.2 \mathrm{mg}, 0.154 \mathrm{mmol}, 77 \%$ yield, $E / Z=54: 46$ ).

${ }^{1} \mathrm{H}$ NMR (400 MHz, $\mathrm{CDCl}_{3}$ ) $\delta 7.30$ (dddd, $J=23.7,22.1,12.1,6.6 \mathrm{~Hz}, 10 \mathrm{H}, Z+E$ ), 6.93 (s, $1 \mathrm{H}, E), 6.78(\mathrm{~s}, 1 \mathrm{H}, Z), 4.35$ (q, $J=7.1 \mathrm{~Hz}, 2 \mathrm{H}, E), 3.81(\mathrm{q}, J=7.2 \mathrm{~Hz}, 2 \mathrm{H}, Z), 2.42(\mathrm{q}, J=$ $7.6 \mathrm{~Hz}, 4 \mathrm{H}, Z+E), 1.36(\mathrm{t}, J=7.1 \mathrm{~Hz}, 3 \mathrm{H}, Z), 1.22(\mathrm{t}, J=7.4 \mathrm{~Hz}, 3 \mathrm{H}, E), 1.17$ (t, $J=7.5 \mathrm{~Hz}$, $3 \mathrm{H}, E), 1.08(\mathrm{t}, J=7.2 \mathrm{~Hz}, 3 \mathrm{H}, Z)$.

${ }^{13} \mathrm{C}$ NMR $\left(101 \mathrm{MHz}, \mathrm{CDCl}_{3}\right) \delta 164.7,164.4,164.0,163.5,135.8,135.2,135.0,133.0,131.7$, 128.9, 128.5, 128.0, 114.9, 113.7, 63.1, 62.7, 25.4, 19.9, 14.0, 13.7, 13.3.

${ }^{19} \mathrm{~F}$ NMR $\left(376 \mathrm{MHz}, \mathrm{CDCl}_{3}\right) \delta-95.68(Z),-104.50(E)$.

HRMS (EI) calcd. for $\mathrm{C}_{14} \mathrm{H}_{16} \mathrm{~F}_{2} \mathrm{O}_{2}{ }^{+}\left(\mathrm{M}^{+}\right)$254.1113, found 254.1116.

\section{Characterization data of difluoroalkylation products}

ethyl $(E)$-2,2-difluoro-4-phenylbut-3-enoate ${ }^{3}$<smiles>CCOC(=O)C(F)(F)F</smiles>

Following general procedure $\mathbf{A}, \mathrm{Cs}_{2} \mathrm{CO}_{3}(\mathrm{MW}=325.8,0.4 \mathrm{mmol}, 130.3 \mathrm{mg}), 4 \mathbf{a}(\mathrm{MW}=$ 104.1, $0.2 \mathrm{mmol}, 20.8 \mathrm{mg}$ ) and $\mathrm{ICF}_{2} \mathrm{COOEt}(\mathrm{MW}=250.0,0.4 \mathrm{mmol}, 100.0 \mathrm{mg}$ ) were added to $\mathrm{CH}_{3} \mathrm{CN}(1 \mathrm{~mL})$, stirred for $12 \mathrm{~h}$ under $30 \mathrm{~W}$ blue LED irradiation at room temperature, the reaction mixture was purified by silica gel chromatography $(\mathrm{PE} / \mathrm{EA}=50: 1)$ to obtain product 5a as colorless liquid (MW =226.1, $29.7 \mathrm{mg}, 0.131 \mathrm{mmol}, 66 \%$ yield, $E / Z>99: 1$ ).

${ }^{1} \mathrm{H}$ NMR (400 MHz, CDCl $) \delta 7.45$ (dd, $\left.J=7.6,1.9 \mathrm{~Hz}, 2 \mathrm{H}\right), 7.41-7.30(\mathrm{~m}, 3 \mathrm{H}), 7.16-6.99$ $(\mathrm{m}, 1 \mathrm{H}), 6.30(\mathrm{dt}, J=16.2,11.4 \mathrm{~Hz}, 1 \mathrm{H}), 4.35(\mathrm{q}, J=7.1 \mathrm{~Hz}, 2 \mathrm{H}), 1.36(\mathrm{t}, J=7.1 \mathrm{~Hz}, 3 \mathrm{H})$.

${ }^{13} \mathrm{C}$ NMR $\left(101 \mathrm{MHz}, \mathrm{CDCl}_{3}\right) \delta 164.3,164.0,163.6,136.9,134.1,129.7,128.9,127.5,112.8$, 110.3, 63.1, 14.0.

${ }^{19} \mathrm{~F}$ NMR $\left(376 \mathrm{MHz}, \mathrm{CDCl}_{3}\right) \delta-103.20$.

\section{ethyl $(E)$-2,2-difluoro-4-(p-tolyl)but-3-enoate ${ }^{3}$<smiles>CCOC(=O)C(F)(F)F</smiles>

Following general procedure $\mathbf{A}, \mathrm{Cs}_{2} \mathrm{CO}_{3}(\mathrm{MW}=325.8,0.4 \mathrm{mmol}, 130.3 \mathrm{mg}), \mathbf{4 b}(\mathrm{MW}=$ $118.1,0.2 \mathrm{mmol}, 23.6 \mathrm{mg}$ ) and $\mathrm{ICF}_{2} \mathrm{COOEt}(\mathrm{MW}=250.0,0.4 \mathrm{mmol}, 100.0 \mathrm{mg}$ ) were added to $\mathrm{CH}_{3} \mathrm{CN}(1 \mathrm{~mL})$, stirred for $15 \mathrm{~h}$ under $30 \mathrm{~W}$ blue LED irradiation at room temperature, the reaction mixture was purified by silica gel chromatography $(\mathrm{PE} / \mathrm{EA}=50: 1)$ to obtain product 5b as colorless liquid ( $\mathrm{MW}=240.1,38.3 \mathrm{mg}, 0.160 \mathrm{mmol}, 80 \%$ yield, $E / Z>99: 1$ ).

${ }^{1} \mathrm{H}$ NMR $\left(400 \mathrm{MHz}, \mathrm{CDCl}_{3}\right) \delta 7.33(\mathrm{~d}, J=8.1 \mathrm{~Hz}, 2 \mathrm{H}), 7.17(\mathrm{~d}, J=8.0 \mathrm{~Hz}, 2 \mathrm{H}), 7.04(\mathrm{dt}, J=$ 16.2, $2.5 \mathrm{~Hz}, 1 \mathrm{H}), 6.25(\mathrm{dt}, J=16.2,11.5 \mathrm{~Hz}, 1 \mathrm{H}), 4.34(\mathrm{q}, J=7.1 \mathrm{~Hz}, 2 \mathrm{H}), 2.35(\mathrm{~s}, 3 \mathrm{H}), 1.35$ (t, $J=7.1 \mathrm{~Hz}, 3 \mathrm{H})$.

${ }^{13} \mathrm{C}$ NMR $\left(101 \mathrm{MHz}, \mathrm{CDCl}_{3}\right) \delta 164.4,164.1,163.7,139.9,136.8,131.4,129.6,127.4,118.0$, $117.8,117.5,115.4,112.9,110.4,63.1,21.3,14.0$.

${ }^{19} \mathrm{~F} \mathrm{NMR}\left(376 \mathrm{MHz}, \mathrm{CDCl}_{3}\right) \delta-102.93$. 
ethyl (E)-2,2-difluoro-4-(4-methoxyphenyl)but-3-enoate ${ }^{3}$

$\overbrace{5 \mathbf{c}}^{C_{2} \text { COOEt }}$

Following general procedure $\mathbf{A}, \mathrm{Cs}_{2} \mathrm{CO}_{3}(\mathrm{MW}=325.8,0.4 \mathrm{mmol}, 130.3 \mathrm{mg}), \mathbf{4 c}(\mathrm{MW}=$ $134.1,0.2 \mathrm{mmol}, 26.8 \mathrm{mg})$ and $\mathrm{ICF}_{2} \mathrm{COOEt}(\mathrm{MW}=250.0,0.4 \mathrm{mmol}, 100.0 \mathrm{mg})$ were added to $\mathrm{CH}_{3} \mathrm{CN}(1 \mathrm{~mL})$, stirred for $15 \mathrm{~h}$ under $30 \mathrm{~W}$ blue LED irradiation at room temperature, the reaction mixture was purified by silica gel chromatography $(\mathrm{PE} / \mathrm{EA}=25: 1)$ to obtain product 5c as colorless liquid (MW $=256.1,31.0 \mathrm{mg}, 0.121 \mathrm{mmol}, 61 \%$ yield, $E / Z>99: 1$ ).

${ }^{1} \mathrm{H}$ NMR $\left(400 \mathrm{MHz}, \mathrm{CDCl}_{3}\right) \delta 7.39(\mathrm{~d}, J=8.8 \mathrm{~Hz}, 2 \mathrm{H}), 7.02(\mathrm{dt}, J=16.2,2.5 \mathrm{~Hz}, 1 \mathrm{H}), 6.89$ (d, $J=8.8 \mathrm{~Hz}, 2 \mathrm{H}), 6.16(\mathrm{dt}, J=16.2,11.5 \mathrm{~Hz}, 1 \mathrm{H}), 4.34(\mathrm{q}, J=7.1 \mathrm{~Hz}, 2 \mathrm{H}), 3.83(\mathrm{~s}, 3 \mathrm{H})$, $1.36(\mathrm{t}, J=7.1 \mathrm{~Hz}, 3 \mathrm{H})$.

${ }^{13} \mathrm{C}$ NMR $\left(101 \mathrm{MHz}, \mathrm{CDCl}_{3}\right) \delta 164.5,164.2,163.8,160.8,136.3,129.0,126.8,116.5,116.3$, 116.0, 115.5, 114.23, 113.0, 110.5, 63.1, 55.4, 14.0 .

${ }^{19} \mathrm{~F}$ NMR $\left(376 \mathrm{MHz}, \mathrm{CDCl}_{3}\right) \delta-102.76$.

ethyl (E)-4-(4-(tert-butyl)phenyl)-2,2-difluorobut-3-enoate ${ }^{7}$<smiles>CCOC(=O)C(F)(F)F</smiles>

Following general procedure $\mathbf{A}, \mathrm{Cs}_{2} \mathrm{CO}_{3}(\mathrm{MW}=325.8,0.4 \mathrm{mmol}, 130.3 \mathrm{mg}), \mathbf{4 d}(\mathrm{MW}=$ $160.1,0.2 \mathrm{mmol}, 32.0 \mathrm{mg}$ ) and $\mathrm{ICF}_{2} \mathrm{COOEt}(\mathrm{MW}=250.0,0.4 \mathrm{mmol}, 100.0 \mathrm{mg})$ were added to $\mathrm{CH}_{3} \mathrm{CN}(1 \mathrm{~mL})$, stirred for $15 \mathrm{~h}$ under $30 \mathrm{~W}$ blue LED irradiation at room temperature, the reaction mixture was purified by silica gel chromatography $(\mathrm{PE} / \mathrm{EA}=50: 1)$ to obtain product 5d as colorless liquid (MW $=282.1,39.0 \mathrm{mg}, 0.138 \mathrm{mmol}, 69 \%$ yield, $E / Z>99: 1$ ).

${ }^{1} \mathrm{H}$ NMR $\left(400 \mathrm{MHz}, \mathrm{CDCl}_{3}\right) \delta 7.39(\mathrm{~s}, 4 \mathrm{H}), 7.06(\mathrm{dt}, J=16.2,2.6 \mathrm{~Hz}, 1 \mathrm{H}), 6.26(\mathrm{dt}, J=16.2$, $11.5 \mathrm{~Hz}, 1 \mathrm{H}), 4.34$ (q, $J=7.1 \mathrm{~Hz}, 2 \mathrm{H}), 1.38-1.29(\mathrm{~m}, 12 \mathrm{H})$.

${ }^{13} \mathrm{C}$ NMR $\left(101 \mathrm{MHz}, \mathrm{CDCl}_{3}\right) \delta 164.4,164.0,163.7,153.1,136.6,131.4,127.3,125.8,118.3$, 118.0, 117.8, 115.4, 112.9, 110.4, 63.1, 34.8, 31.2, 14.0.

${ }^{19} \mathrm{~F}$ NMR $\left(376 \mathrm{MHz}, \mathrm{CDCl}_{3}\right) \delta-103.06$.

ethyl $(E)$-2,2-difluoro-4-(4-fluorophenyl)but-3-enoate ${ }^{3}$<smiles>CCOC(=O)C(F)(F)F</smiles>

Following general procedure $\mathbf{A}, \mathrm{Cs}_{2} \mathrm{CO}_{3}(\mathrm{MW}=325.8,0.4 \mathrm{mmol}, 130.3 \mathrm{mg}), \mathbf{4 e}(\mathrm{MW}=$ $122.1,0.2 \mathrm{mmol}, 24.4 \mathrm{mg}$ ) and $\mathrm{ICF}_{2} \mathrm{COOEt}(\mathrm{MW}=250.0,0.4 \mathrm{mmol}, 100.0 \mathrm{mg}$ ) were added to $\mathrm{CH}_{3} \mathrm{CN}(1 \mathrm{~mL})$, stirred for $13 \mathrm{~h}$ under $30 \mathrm{~W}$ blue LED irradiation at room temperature, the reaction mixture was purified by silica gel chromatography (PE/EA $=50: 1)$ to obtain product 5e as colorless liquid (MW $=244.1,36.9 \mathrm{mg}, 0.151 \mathrm{mmol}, 76 \%$ yield, $E / Z>99: 1$ ).

${ }^{1} \mathrm{H}$ NMR $\left(400 \mathrm{MHz}, \mathrm{CDCl}_{3}\right) \delta 7.43(\mathrm{dd}, J=8.7,5.4 \mathrm{~Hz}, 2 \mathrm{H}), 7.16-6.92(\mathrm{~m}, 3 \mathrm{H}), 6.23(\mathrm{dt}, J$ $=16.2,11.3 \mathrm{~Hz}, 1 \mathrm{H}), 4.35(\mathrm{q}, J=7.1 \mathrm{~Hz}, 2 \mathrm{H}), 1.37(\mathrm{t}, J=7.1 \mathrm{~Hz}, 3 \mathrm{H})$.

${ }^{13} \mathrm{C}$ NMR $\left(101 \mathrm{MHz}, \mathrm{CDCl}_{3}\right) \delta 164.7,164.2,163.9,163.5,162.3,135.6,130.4,129.3,118.9$, 118.6, 118.4, 116.1, 115.8, 115.1, 112.6, 110.2, 63.2, 14.0. 
${ }^{19} \mathrm{~F}$ NMR $\left(376 \mathrm{MHz}, \mathrm{CDCl}_{3}\right) \delta-103.16,-110.91$.

\section{ethyl $(E)-2,2-d i f l u o r o-4-(3-f l u o r o p h e n y l) b u t-3-$ enoate $^{3}$ \\ $\mathrm{CF}_{\mathbf{5 f}} \mathrm{COOEt}$}

Following general procedure $\mathbf{A}, \mathrm{Cs}_{2} \mathrm{CO}_{3}(\mathrm{MW}=325.8,0.4 \mathrm{mmol}, 130.3 \mathrm{mg}), \mathbf{4 f}(\mathrm{MW}=$ 122.1, $0.2 \mathrm{mmol}, 24.4 \mathrm{mg}$ ) and $\mathrm{ICF}_{2} \mathrm{COOEt}(\mathrm{MW}=250.0,0.4 \mathrm{mmol}, 100.0 \mathrm{mg}$ ) were added to $\mathrm{CH}_{3} \mathrm{CN}(1 \mathrm{~mL})$, stirred for $15 \mathrm{~h}$ under $30 \mathrm{~W}$ blue LED irradiation at room temperature, the reaction mixture was purified by silica gel chromatography $(\mathrm{PE} / \mathrm{EA}=50: 1)$ to obtain product 5f as colorless liquid ( $\mathrm{MW}=244.1,32.7 \mathrm{mg}, 0.134 \mathrm{mmol}, 67 \%$ yield, $E / Z>98: 2$ ).

${ }^{1} \mathrm{H}$ NMR $\left(400 \mathrm{MHz}, \mathrm{CDCl}_{3}\right) \delta 7.35(\mathrm{td}, J=8.0,5.9 \mathrm{~Hz}, 1 \mathrm{H}), 7.22(\mathrm{~d}, J=7.8 \mathrm{~Hz}, 1 \mathrm{H}), 7.19-$ $7.12(\mathrm{~m}, 1 \mathrm{H}), 7.05$ (ddd, $J=13.1,5.0,2.5 \mathrm{~Hz}, 2 \mathrm{H}), 6.31(\mathrm{dt}, J=16.2,11.3 \mathrm{~Hz}, 1 \mathrm{H}), 4.36(\mathrm{q}, J$ $=7.1 \mathrm{~Hz}, 2 \mathrm{H}), 1.37(\mathrm{t}, J=7.1 \mathrm{~Hz}, 3 \mathrm{H})$.

${ }^{13} \mathrm{C}$ NMR $\left(101 \mathrm{MHz}, \mathrm{CDCl}_{3}\right) \delta 164.2,163.8,161.8,136.3,135.7,130.5,123.5,120.5,120.2$, $120.0,116.7,116.5,114.9,114.0,113.8,112.5,110.0,63.3,14.0$.

${ }^{19} \mathrm{~F}$ NMR $\left(376 \mathrm{MHz}, \mathrm{CDCl}_{3}\right) \delta-103.63,-112.59$.

\section{ethyl $(E)-2,2-d i f l u o r o-4-(2-f l u o r o p h e n y l) b u t-3-$ enoate $^{3}$<smiles>CCOC(=O)C(F)(F)F</smiles>

Following general procedure $\mathbf{A}, \mathrm{Cs}_{2} \mathrm{CO}_{3}(\mathrm{MW}=325.8,0.4 \mathrm{mmol}, 130.3 \mathrm{mg}), \mathbf{4 g}(\mathrm{MW}=$ $122.1,0.2 \mathrm{mmol}, 24.4 \mathrm{mg}$ ) and $\mathrm{ICF}_{2} \mathrm{COOEt}(\mathrm{MW}=250.0,0.4 \mathrm{mmol}, 100.0 \mathrm{mg}$ ) were added to $\mathrm{CH}_{3} \mathrm{CN}(1 \mathrm{~mL})$, stirred for $15 \mathrm{~h}$ under $30 \mathrm{~W}$ blue LED irradiation at room temperature, the reaction mixture was purified by silica gel chromatography $(\mathrm{PE} / \mathrm{EA}=50: 1)$ to obtain product $\mathbf{5 g}$ as colorless liquid (MW = 244.1, $37.9 \mathrm{mg}, 0.155 \mathrm{mmol}, 78 \%$ yield, $E / \mathrm{Z}>99: 1$ ).

${ }^{1} \mathrm{H}$ NMR (400 MHz, $\left.\mathrm{CDCl}_{3}\right) \delta 7.47(\mathrm{td}, J=7.6,1.4 \mathrm{~Hz}, 1 \mathrm{H}), 7.34(\mathrm{td}, J=7.4,1.5 \mathrm{~Hz}, 1 \mathrm{H})$, $7.21(\mathrm{dt}, J=16.4,2.6 \mathrm{~Hz}, 1 \mathrm{H}), 7.18-7.05(\mathrm{~m}, 2 \mathrm{H}), 6.43(\mathrm{dt}, J=16.4,11.3 \mathrm{~Hz}, 1 \mathrm{H}), 4.36(\mathrm{q}$, $J=7.1 \mathrm{~Hz}, 2 \mathrm{H}), 1.37(\mathrm{t}, J=7.1 \mathrm{~Hz}, 3 \mathrm{H})$.

${ }^{13} \mathrm{C}$ NMR $\left(101 \mathrm{MHz}, \mathrm{CDCl}_{3}\right) \delta 164.2,163.8,163.5,162.2,159.7,131.2,129.8,128.6,124.4$, 122.0, 121.4, 116.3, 116.0, 112.6, 110.1, 63.3, 14.0.

${ }^{19} \mathrm{~F}$ NMR $\left(376 \mathrm{MHz}, \mathrm{CDCl}_{3}\right) \delta-103.78,-115.67$.

\section{ethyl (E)-4-(4-chlorophenyl)-2,2-difluorobut-3-enoate ${ }^{3}$}<smiles>CCOC(=O)C(F)(F)F</smiles>

Following general procedure $\mathbf{A}, \mathrm{Cs}_{2} \mathrm{CO}_{3}(\mathrm{MW}=325.8,0.4 \mathrm{mmol}, 130.3 \mathrm{mg}), \mathbf{4 h}(\mathrm{MW}=$ 138.0, $0.2 \mathrm{mmol}, 27.6 \mathrm{mg}$ ) and $\mathrm{ICF}_{2} \mathrm{COOEt}(\mathrm{MW}=250.0,0.4 \mathrm{mmol}, 100.0 \mathrm{mg}$ ) were added to $\mathrm{CH}_{3} \mathrm{CN}(1 \mathrm{~mL})$, stirred for $15 \mathrm{~h}$ under $30 \mathrm{~W}$ blue LED irradiation at room temperature, the reaction mixture was purified by silica gel chromatography $(\mathrm{PE} / \mathrm{EA}=50: 1)$ to obtain product 5h as colorless liquid (MW = 260.0, $40.9 \mathrm{mg}, 0.157 \mathrm{mmol}, 79 \%$ yield, $E / Z>99: 1$ ). 
${ }^{1} \mathrm{H}$ NMR $\left(400 \mathrm{MHz}, \mathrm{CDCl}_{3}\right) \delta 7.44-7.30(\mathrm{~m}, 4 \mathrm{H}), 7.03(\mathrm{dt}, J=16.2,2.5 \mathrm{~Hz}, 1 \mathrm{H}), 6.28(\mathrm{dt}, J$ $=16.2,11.3 \mathrm{~Hz}, 1 \mathrm{H}), 4.35(\mathrm{q}, J=7.1 \mathrm{~Hz}, 2 \mathrm{H}), 1.36(\mathrm{t}, J=7.1 \mathrm{~Hz}, 3 \mathrm{H})$.

${ }^{13} \mathrm{C}$ NMR $\left(101 \mathrm{MHz}, \mathrm{CDCl}_{3}\right) \delta 164.1,163.8,163.4,135.6,132.6,129.1,128.7,119.7,119.5$, 119.2, 115.0, 112.6, 110.1, 63.2, 14.0.

${ }^{19} \mathrm{~F}$ NMR $\left(376 \mathrm{MHz}, \mathrm{CDCl}_{3}\right) \delta-103.31$.

ethyl (E)-4-(4-bromophenyl)-2,2-difluorobut-3-enoate ${ }^{6}$<smiles>CCOC(=O)C(F)(F)F</smiles>

Following general procedure $\mathbf{A}, \mathrm{Cs}_{2} \mathrm{CO}_{3}(\mathrm{MW}=325.8,0.4 \mathrm{mmol}, 130.3 \mathrm{mg}), \mathbf{4 i}(\mathrm{MW}=182.0$, $0.2 \mathrm{mmol}, 36.4 \mathrm{mg}$ ) and $\mathrm{ICF}_{2} \mathrm{COOEt}(\mathrm{MW}=250.0,0.4 \mathrm{mmol}, 100.0 \mathrm{mg}$ ) were added to $\mathrm{CH}_{3} \mathrm{CN}(1 \mathrm{~mL})$, stirred for $15 \mathrm{~h}$ under $30 \mathrm{~W}$ blue LED irradiation at room temperature, the reaction mixture was purified by silica gel chromatography $(\mathrm{PE} / \mathrm{EA}=50: 1)$ to obtain product $\mathbf{5 i}$ as colorless liquid ( $\mathrm{MW}=304.0,43.0 \mathrm{mg}, 0.141 \mathrm{mmol}, 71 \%$ yield, $E / Z>98: 2$ ).

${ }^{1} \mathrm{H}$ NMR $\left(400 \mathrm{MHz}, \mathrm{CDCl}_{3}\right) \delta 7.50(\mathrm{~d}, J=8.5 \mathrm{~Hz}, 2 \mathrm{H}), 7.32(\mathrm{~d}, J=8.5 \mathrm{~Hz}, 2 \mathrm{H}), 7.02(\mathrm{dd}, J=$ $16.2,2.5 \mathrm{~Hz}, 1 \mathrm{H}), 6.30(\mathrm{dt}, J=16.2,11.4 \mathrm{~Hz}, 1 \mathrm{H}), 4.36(\mathrm{q}, J=7.1 \mathrm{~Hz}, 2 \mathrm{H}), 1.37(\mathrm{t}, J=7.1$ $\mathrm{Hz}, 3 \mathrm{H})$.

${ }^{13} \mathrm{C}$ NMR $\left(101 \mathrm{MHz}, \mathrm{CDCl}_{3}\right) \delta 164.1,163.8,163.5,135.7,133.0,132.1,129.0,123.8,119.7$, 119.5, 119.2, 115.0, 112.6, 110.1, 63.3, 14.0.

${ }^{19} \mathrm{~F} \mathrm{NMR}\left(376 \mathrm{MHz}, \mathrm{CDCl}_{3}\right) \delta-103.47$.

ethyl (E)-2,2-difluoro-4-(naphthalen-2-yl)but-3-enoate ${ }^{6}$<smiles>CCOC(=O)C(F)(F)F</smiles>

Following general procedure $\mathbf{A}, \mathrm{Cs}_{2} \mathrm{CO}_{3}(\mathrm{MW}=325.8,0.4 \mathrm{mmol}, 130.3 \mathrm{mg}), \mathbf{4 j}(\mathrm{MW}=$ $154.1,0.2 \mathrm{mmol}, 30.8 \mathrm{mg}$ ) and $\mathrm{ICF}_{2} \mathrm{COOEt}(\mathrm{MW}=250.0,0.4 \mathrm{mmol}, 100.0 \mathrm{mg}$ ) were added to $\mathrm{CH}_{3} \mathrm{CN}(1 \mathrm{~mL})$, stirred for $15 \mathrm{~h}$ under $30 \mathrm{~W}$ blue LED irradiation at room temperature, the reaction mixture was purified by silica gel chromatography $(\mathrm{PE} / \mathrm{EA}=40: 1)$ to obtain product $\mathbf{5 j}$ as white solid ( $\mathrm{MW}=276.1,38.4 \mathrm{mg}, 0.139 \mathrm{mmol}, 70 \%$ yield, $E / Z>99: 1)$.

${ }^{1} \mathrm{H}$ NMR $\left(400 \mathrm{MHz}, \mathrm{CDCl}_{3}\right) \delta 7.81(\mathrm{dd}, J=6.0,2.5 \mathrm{~Hz}, 4 \mathrm{H}), 7.62-7.55(\mathrm{~m}, 1 \mathrm{H}), 7.49(\mathrm{dd}, J$ $=6.2,3.3 \mathrm{~Hz}, 2 \mathrm{H}), 7.23(\mathrm{dt}, J=16.1,2.4 \mathrm{~Hz}, 1 \mathrm{H}), 6.42(\mathrm{dt}, J=16.2,11.5 \mathrm{~Hz}, 1 \mathrm{H}), 4.36(\mathrm{q}, J$ $=7.1 \mathrm{~Hz}, 2 \mathrm{H}), 1.37(\mathrm{t}, J=7.1 \mathrm{~Hz}, 3 \mathrm{H})$.

${ }^{13} \mathrm{C}$ NMR $\left(101 \mathrm{MHz}, \mathrm{CDCl}_{3}\right) \delta 164.4,164.1,163.7,137.0,133.9,133.3,131.5,128.9,128.7$, 128.4, 127.8, 127.0, 126.8, 123.3, 119.2, 118.9, 118.7, 115.4, 112.9, 110.4, 63.3, 14.0.

${ }^{19} \mathrm{~F}$ NMR $\left(376 \mathrm{MHz}, \mathrm{CDCl}_{3}\right) \delta-103.07$.

ethyl 2,2-difluoro-4,4-diphenylbut-3-enoate ${ }^{6}$<smiles>CCOC(=O)C(F)(F)C=C(c1ccccc1)c1ccccc1</smiles>

Following general procedure $\mathbf{A}, \mathrm{Cs}_{2} \mathrm{CO}_{3}(\mathrm{MW}=325.8,0.4 \mathrm{mmol}, 130.3 \mathrm{mg}), \mathbf{4 k}(\mathrm{MW}=$ 
180.1, $0.2 \mathrm{mmol}, 36.0 \mathrm{mg})$ and $\mathrm{ICF}_{2} \mathrm{COOEt}(\mathrm{MW}=250.0,0.4 \mathrm{mmol}, 100.0 \mathrm{mg})$ were added to $\mathrm{CH}_{3} \mathrm{CN}(1 \mathrm{~mL})$, stirred for $14 \mathrm{~h}$ under $30 \mathrm{~W}$ blue LED irradiation at room temperature, the reaction mixture was purified by silica gel chromatography $(\mathrm{PE} / \mathrm{EA}=40: 1)$ to obtain product 5k as colorless liquid ( $\mathrm{MW}=302.1,53.4 \mathrm{mg}, 0.177 \mathrm{mmol}, 89 \%$ yield).

${ }^{1} \mathrm{H}$ NMR (400 MHz, $\left.\mathrm{CDCl}_{3}\right) \delta 7.48-7.10(\mathrm{~m}, 10 \mathrm{H}), 6.27(\mathrm{t}, J=11.7 \mathrm{~Hz}, 1 \mathrm{H}), 3.89(\mathrm{q}, J=$ $7.2 \mathrm{~Hz}, 2 \mathrm{H}), 1.16(\mathrm{t}, J=7.2 \mathrm{~Hz}, 3 \mathrm{H})$.

${ }^{13} \mathrm{C}$ NMR $\left(101 \mathrm{MHz}, \mathrm{CDCl}_{3}\right) \delta 163.8,163.5,163.2,151.0,140.5,137.1,129.9,129.2,128.7$, 128.5, 128.0, 119.8, 119.5, 119.2, 115.0, 112.6, 110.2, 62.8, 13.7 .

${ }^{19} \mathrm{~F}$ NMR $\left(376 \mathrm{MHz}, \mathrm{CDCl}_{3}\right) \delta-90.75$.

\section{2-((ethylperoxy)difluoromethyl)benzofuran 6}

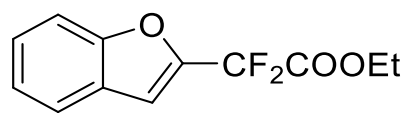

5I

Following general procedure $\mathbf{A}, \mathrm{Cs}_{2} \mathrm{CO}_{3}(\mathrm{MW}=325.8,0.4 \mathrm{mmol}, 130.3 \mathrm{mg}), \mathbf{4 l}(\mathrm{MW}=118.0$, $0.2 \mathrm{mmol}, 23.6 \mathrm{mg})$ and $\mathrm{ICF}_{2} \mathrm{COOEt}(\mathrm{MW}=250.0,0.4 \mathrm{mmol}, 100.0 \mathrm{mg})$ were added to $\mathrm{CH}_{3} \mathrm{CN}$ (1mL), stirred for $15 \mathrm{~h}$ under $30 \mathrm{~W}$ blue LED irradiation at room temperature, the reaction mixture was purified by silica gel chromatography $(\mathrm{PE} / \mathrm{EA}=40: 1)$ to obtain product $\mathbf{5 l}$ as colorless liquid ( $\mathrm{MW}=240.1,32.6 \mathrm{mg}, 0.136 \mathrm{mmol}, 68 \%$ yield).

${ }^{1} \mathrm{H}$ NMR $\left(400 \mathrm{MHz}, \mathrm{CDCl}_{3}\right) \delta 7.73-7.61(\mathrm{~m}, 1 \mathrm{H}), 7.59-7.50(\mathrm{~m}, 1 \mathrm{H}), 7.41(\mathrm{dd}, J=13.7$, $6.9 \mathrm{~Hz}, 1 \mathrm{H}), 7.36-7.27(\mathrm{~m}, 1 \mathrm{H}), 7.16(\mathrm{~d}, J=3.6 \mathrm{~Hz}, 1 \mathrm{H}), 4.52-4.33(\mathrm{~m}, 2 \mathrm{H}), 1.46-1.32$ $(\mathrm{m}, 3 \mathrm{H})$.

${ }^{13} \mathrm{C}$ NMR $\left(101 \mathrm{MHz}, \mathrm{CDCl}_{3}\right) \delta 162.6,162.2,161.9,155.4,146.4,126.5,123.8,122.3,112.1$, 111.4, 109.0, 108.2, 106.5, 63.8, 14.0.

${ }^{19} \mathrm{~F}$ NMR $\left(376 \mathrm{MHz}, \mathrm{CDCl}_{3}\right) \delta-104.17$.

ethyl 2,2-difluoro-2-(2-oxo-2H-chromen-3-yl)acetate ${ }^{6}$<smiles>CCOC(=O)C(F)(F)c1cc2ccccc2oc1=O</smiles>

Following general procedure $\mathbf{A}, \mathrm{Cs}_{2} \mathrm{CO}_{3}(\mathrm{MW}=325.8,0.4 \mathrm{mmol}, 130.3 \mathrm{mg}), \mathbf{4 m}(\mathrm{MW}=$ 146.0, $0.2 \mathrm{mmol}, 29.2 \mathrm{mg}$ ) and $\mathrm{ICF}_{2} \mathrm{COOEt}(\mathrm{MW}=250.0,0.4 \mathrm{mmol}, 100.0 \mathrm{mg}$ ) were added to $\mathrm{CH}_{3} \mathrm{CN}(1 \mathrm{~mL})$, stirred for $15 \mathrm{~h}$ under $30 \mathrm{~W}$ blue LED irradiation at room temperature, the reaction mixture was purified by silica gel chromatography $(\mathrm{PE} / \mathrm{EA}=15: 1)$ to obtain product $\mathbf{5 m}$ as white solid (MW $=268.1,33.8 \mathrm{mg}, 0.126 \mathrm{mmol}, 63 \%$ yield).

${ }^{1} \mathrm{H}$ NMR (400 MHz, $\left.\mathrm{CDCl}_{3}\right) \delta 8.19(\mathrm{~s}, 1 \mathrm{H}), 7.71-7.55(\mathrm{~m}, 2 \mathrm{H}), 7.38(\mathrm{dd}, J=11.4,4.3 \mathrm{~Hz}$, $2 \mathrm{H}), 4.40(\mathrm{q}, J=7.1 \mathrm{~Hz}, 2 \mathrm{H}), 1.35(\mathrm{t}, J=7.1 \mathrm{~Hz}, 3 \mathrm{H})$.

${ }^{13} \mathrm{C}$ NMR $\left(101 \mathrm{MHz}, \mathrm{CDCl}_{3}\right) \delta 162.6,162.3,162.0,158.0,154.2,142.0,133.8,129.3,125.3$, $121.4,121.2,120.9,117.5,117.0,113.0,110.5,108.0,63.6,13.9$.

${ }^{19} \mathrm{~F} \mathrm{NMR}\left(376 \mathrm{MHz}, \mathrm{CDCl}_{3}\right) \delta-106.15$.

\section{ethyl 2-(3,4-dihydro-2H-pyran-5-yl)-2,2-difluoroacetate ${ }^{6}$}




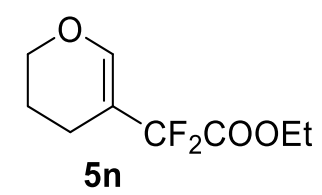

Following general procedure $\mathbf{A}, \mathrm{Cs}_{2} \mathrm{CO}_{3}(\mathrm{MW}=325.8,0.4 \mathrm{mmol}, 130.3 \mathrm{mg}), \mathbf{4 n}(\mathrm{MW}=84.1$, $0.2 \mathrm{mmol}, 16.8 \mathrm{mg}$ ) and $\mathrm{ICF}_{2} \mathrm{COOEt}(\mathrm{MW}=250.0,0.4 \mathrm{mmol}, 100.0 \mathrm{mg}$ ) were added to $\mathrm{CH}_{3} \mathrm{CN}(1 \mathrm{~mL})$, stirred for $15 \mathrm{~h}$ under $30 \mathrm{~W}$ blue LED irradiation at room temperature, the reaction mixture was purified by silica gel chromatography $(\mathrm{PE} / \mathrm{EA}=50: 1)$ to obtain product 5n as colorless liquid (MW = 206.1, $18.2 \mathrm{mg}, 0.088 \mathrm{mmol}, 44 \%$ yield).

${ }^{1} \mathrm{H}$ NMR $\left(400 \mathrm{MHz}, \mathrm{CDCl}_{3}\right) \delta 6.85(\mathrm{~s}, 1 \mathrm{H}), 4.33(\mathrm{q}, J=7.1 \mathrm{~Hz}, 2 \mathrm{H}), 4.05-3.97(\mathrm{~m}, 2 \mathrm{H})$, $2.14(\mathrm{td}, J=6.3,1.4 \mathrm{~Hz}, 2 \mathrm{H}), 1.99-1.79(\mathrm{~m}, 2 \mathrm{H}), 1.35(\mathrm{t}, J=7.1 \mathrm{~Hz}, 3 \mathrm{H})$.

${ }^{13} \mathrm{C}$ NMR (101 MHz, $\left.\mathrm{CDCl}_{3}\right) \delta 164.1,146.4,114.3,105.9,66.0,62.8,21.1,17.9,14.0$.

${ }^{19} \mathrm{~F} \mathrm{NMR}\left(376 \mathrm{MHz}, \mathrm{CDCl}_{3}\right) \delta-104.77$.

ethyl (E)-2,2-difluorododec-3-enoate ${ }^{6}$

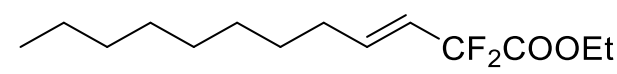

50

Following general procedure $\mathbf{A}, \mathrm{Cs}_{2} \mathrm{CO}_{3}(\mathrm{MW}=325.8,0.4 \mathrm{mmol}, 130.3 \mathrm{mg}), 4 \mathrm{o}(\mathrm{MW}=$ $140.2,0.2 \mathrm{mmol}, 28.0 \mathrm{mg}$ ) and $\mathrm{ICF}_{2} \mathrm{COOEt}(\mathrm{MW}=250.0,0.4 \mathrm{mmol}, 100.0 \mathrm{mg}$ ) were added to $\mathrm{CH}_{3} \mathrm{CN}(1 \mathrm{~mL})$, stirred for $28 \mathrm{~h}$ under $30 \mathrm{~W}$ blue LED irradiation at room temperature, the reaction mixture was purified by silica gel chromatography $(\mathrm{PE} / \mathrm{EA}=70: 1)$ to obtain product 5o as colorless liquid ( $\mathrm{MW}=262.2,38.2 \mathrm{mg}, 0.146 \mathrm{mmol}, 73 \%$ yield).

${ }^{1} \mathrm{H}$ NMR $\left(400 \mathrm{MHz}, \mathrm{CDCl}_{3}\right) \delta 6.38-6.11(\mathrm{~m}, 1 \mathrm{H}), 5.79-5.56(\mathrm{~m}, 1 \mathrm{H}), 4.32(\mathrm{q}, J=7.1 \mathrm{~Hz}$, $2 \mathrm{H}), 2.22-2.06(\mathrm{~m}, 2 \mathrm{H}), 1.46-1.40(\mathrm{~m}, 2 \mathrm{H}), 1.35(\mathrm{t}, J=7.1 \mathrm{~Hz}, 3 \mathrm{H}), 1.27(\mathrm{~s}, 10 \mathrm{H}), 0.88(\mathrm{t}$, $J=6.9 \mathrm{~Hz}, 3 \mathrm{H})$.

${ }^{13} \mathrm{C}$ NMR $\left(101 \mathrm{MHz}, \mathrm{CDCl}_{3}\right) \delta 164.3,140.1,121.1,120.8,120.6,114.9,112.4,62.9,31.9$, $29.7-29.0,28.1,22.7,14.0$.

${ }^{19} \mathrm{~F}$ NMR $\left(376 \mathrm{MHz}, \mathrm{CDCl}_{3}\right) \delta-98.85(Z),-103.00(E)$.

\section{ethyl 2,2-difluoro-4-iodododecanoate ${ }^{8}$}

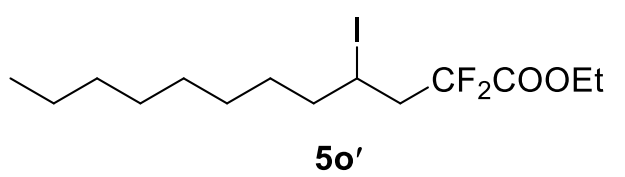

Following general procedure $\mathbf{A}, \mathrm{Cs}_{2} \mathrm{CO}_{3}(\mathrm{MW}=325.8,0.4 \mathrm{mmol}, 130.3 \mathrm{mg}), \mathbf{4 o}(\mathrm{MW}=$ $140.2,0.2 \mathrm{mmol}, 28.0 \mathrm{mg}$ ) and $\mathrm{ICF}_{2} \mathrm{COOEt}(\mathrm{MW}=250.0,0.4 \mathrm{mmol}, 100.0 \mathrm{mg}$ ) were added to $\mathrm{CH}_{3} \mathrm{CN}(1 \mathrm{~mL})$, stirred for $10 \mathrm{~h}$ under $30 \mathrm{~W}$ blue LED irradiation at room temperature, the reaction mixture was purified by silica gel chromatography $(\mathrm{PE} / \mathrm{EA}=70: 1)$ to obtain product 5o' $^{\prime}$ as colorless liquid (MW = 390.1, $57.6 \mathrm{mg}, 0.148 \mathrm{mmol}, 74 \%$ yield).

${ }^{1} \mathrm{H}$ NMR (400 MHz, CDCl3) $\delta 4.35$ (q, J = 7.2 Hz, 2H), $4.29-4.14(\mathrm{~m}, 1 \mathrm{H}), 2.92$ (dtd, J = 18.2, 16.1, $6.6 \mathrm{~Hz}, 1 \mathrm{H}$ ), 2.74 (dddd, $J=17.7,15.6,12.6,7.1 \mathrm{~Hz}, 1 \mathrm{H}$ ), 1.76 (dddd, $J=33.0$, $14.5,9.8,4.9 \mathrm{~Hz}, 2 \mathrm{H}), 1.59-1.46(\mathrm{~m}, 1 \mathrm{H}), 1.44-1.21(\mathrm{~m}, 14 \mathrm{H}), 0.88(\mathrm{t}, J=6.9 \mathrm{~Hz}, 3 \mathrm{H})$. 
${ }^{13} \mathrm{C}$ NMR $\left(101 \mathrm{MHz}, \mathrm{CDCl}_{3}\right) \delta 163.8,163.5,163.2,117.7,115.2,112.7,63.3,45.6,45.3,45.1$, 40.4, 31.8, $29.6-29.1,28.6,23.4,22.7,14.1,13.9$.

${ }^{19} \mathrm{~F}$ NMR $\left(376 \mathrm{MHz}, \mathrm{CDCl}_{3}\right) \delta-101.74,-102.43,-106.53,-107.23$.

\section{Reference}

[1] Guo, W.-H.; Zhao, H.-Y.; Luo, Z.-J.; Zhang, S.; Zhang, X. ACS Catal. 2019, 9, 38-43.

[2] Wang, S.; Zhang, J.; Kong, L.; Tan, Z.; Bai, Y.; Zhu, G. Org. Lett. 2018, 20, 5631-5635.

[3] Zhong, J.-J.; Yang, C.; Chang, X.-Y.; Zou, C.; Lu, W.; Che, C.-M. Chem. Comm.. 2017, $53,8948-8951$.

[4] Li, G.; Cao, Y.-X.; Luo, C.-G.; Su, Y.-M.; Li, Y.; Lan, Q.; Wang, X.-S. Org. Lett. 2018, $18,4806-4809$.

[5] Ma, J.-J.; Yi, W.-B. Org. Biomol. Chem. 2017, 15, 4295-4299.

[6] Wang, X.; Zhao, S.; Liu, J.; Zhu, D.; Guo, M.; Tang, X.; Wang, G. Org. Lett. 2017, 19, 4187-4190.

[7] Yu, C.; Iqbal, N.; Park, S.; Cho, E. J. Chem. Comm. 2014, 50, 12884-12887.

[8] Yajima, T.; Ikegami, M. Eur. J. Org. Chem. 2017, 2126-2129.

\section{NMR spectra}

LKK-3-22-3<smiles>CCOC(=O)C(C)=C(C)c1ccccc1</smiles>

2a

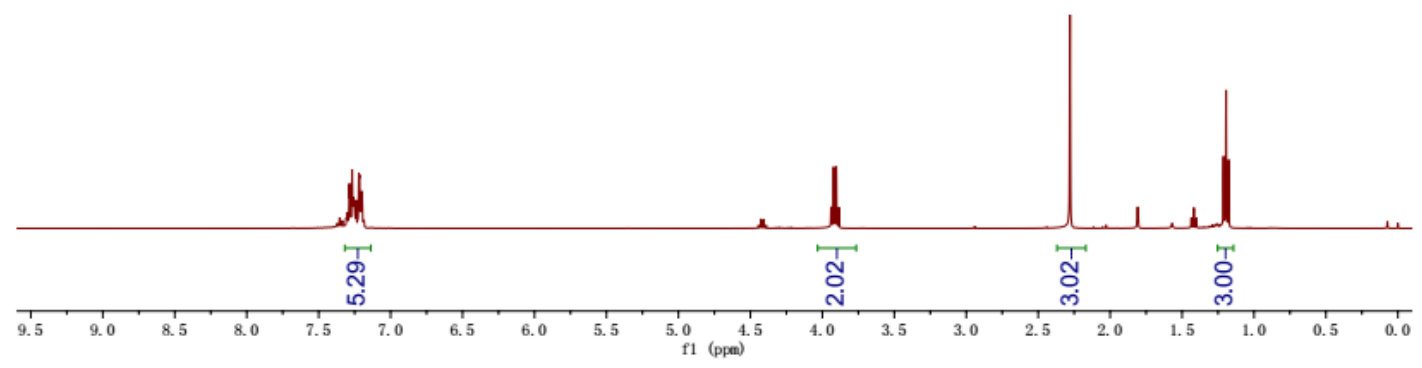



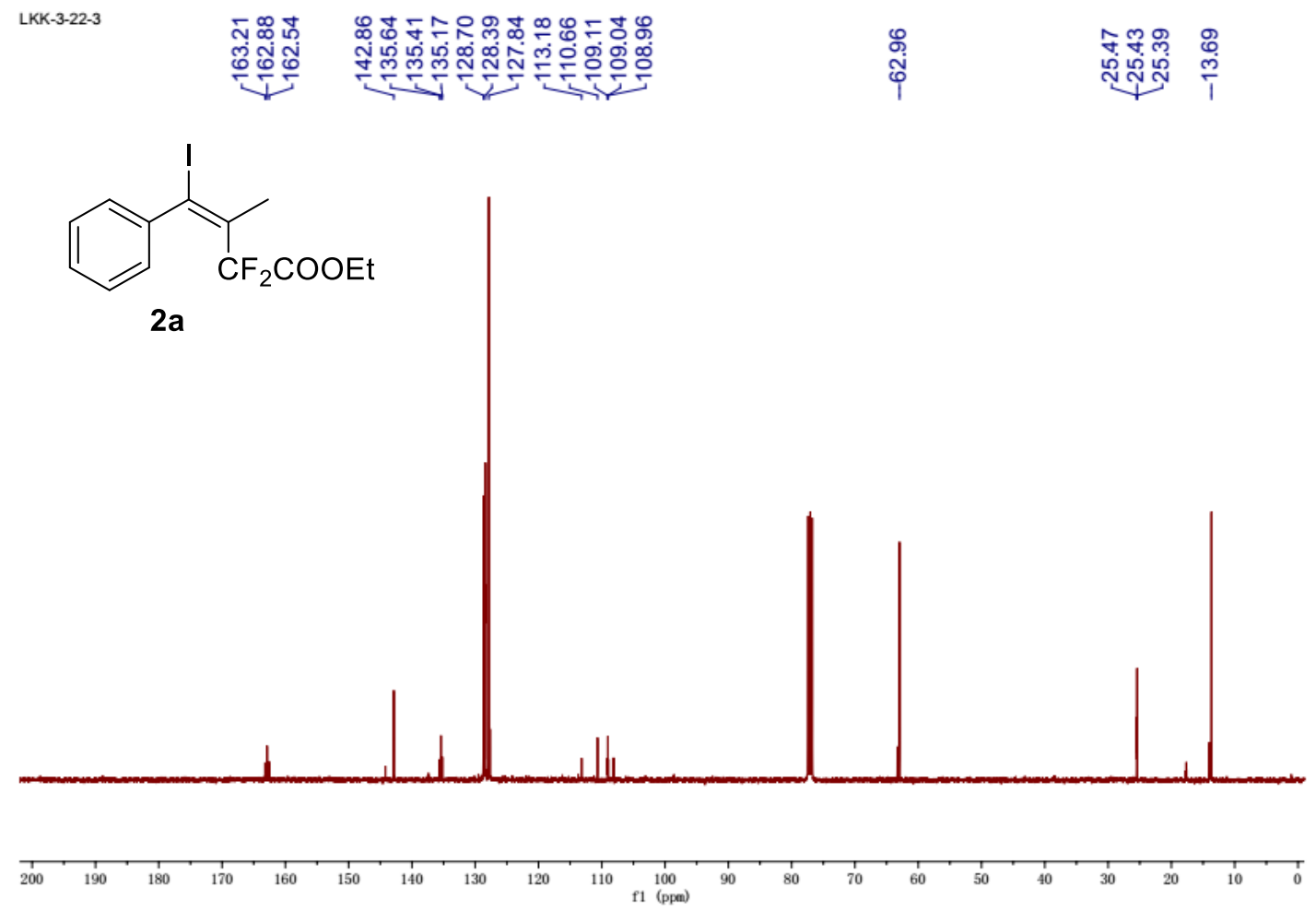

LKK-3-22-3

旁量<smiles>CCOC(=O)C(F)(F)/C(C)=C(/I)c1ccccc1</smiles>

2a

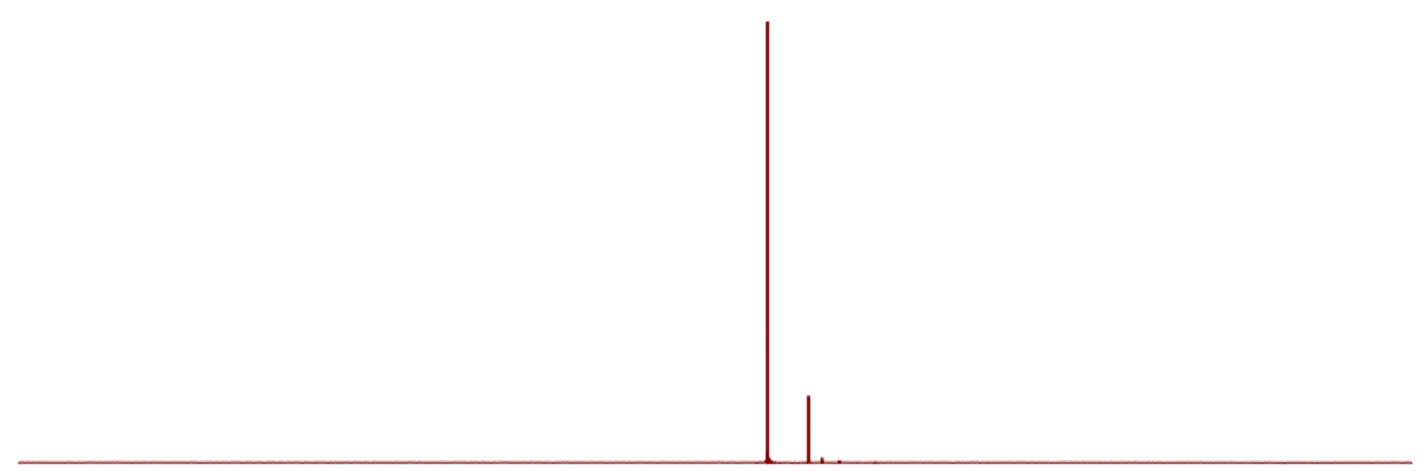

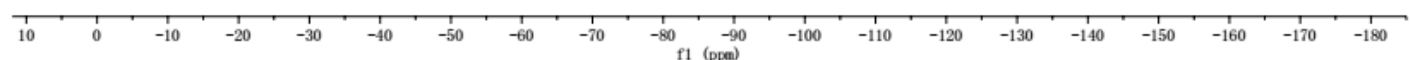




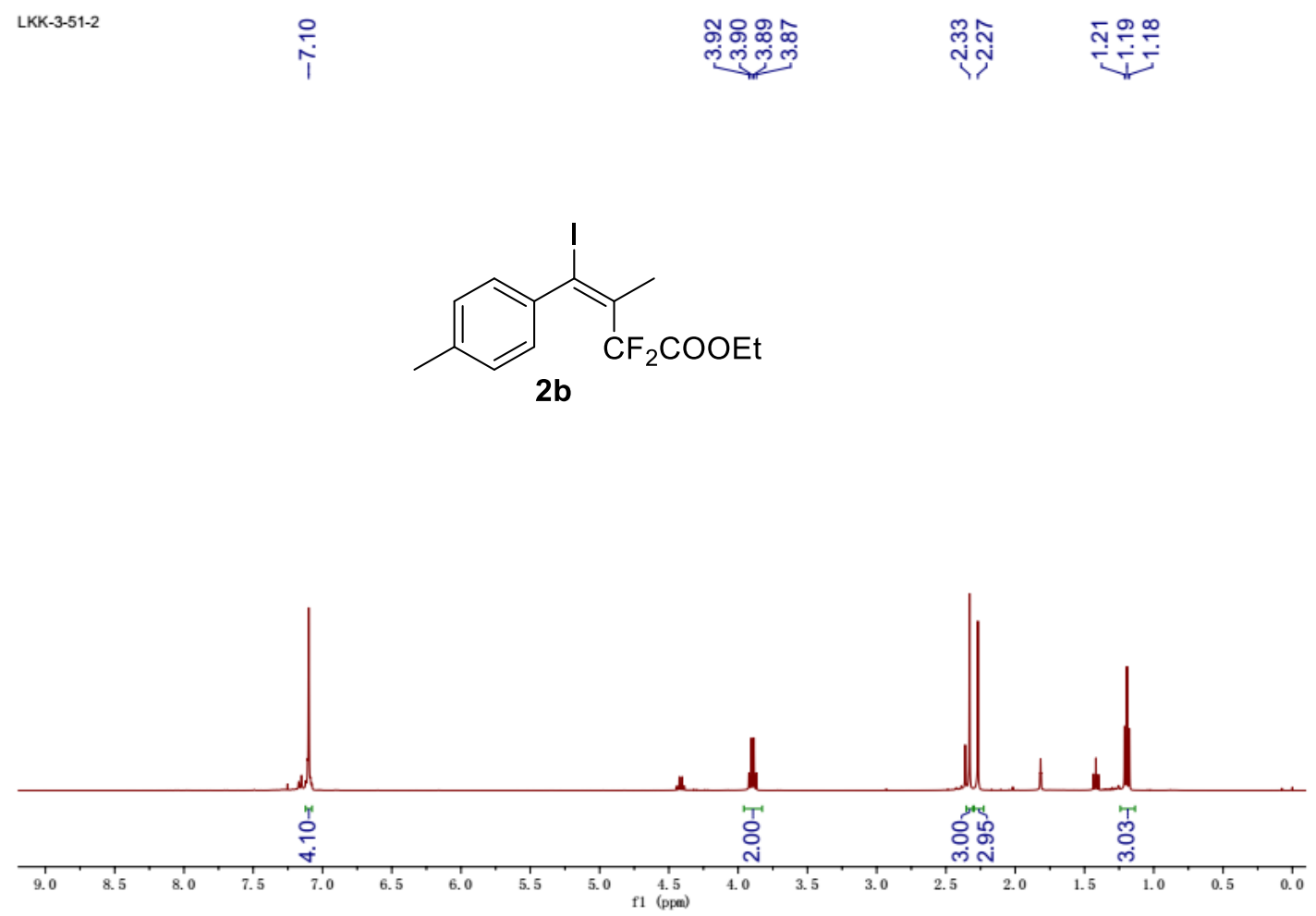

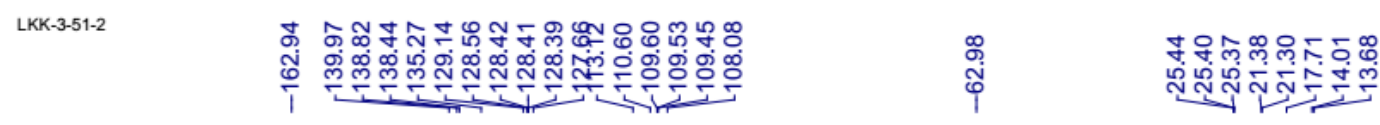

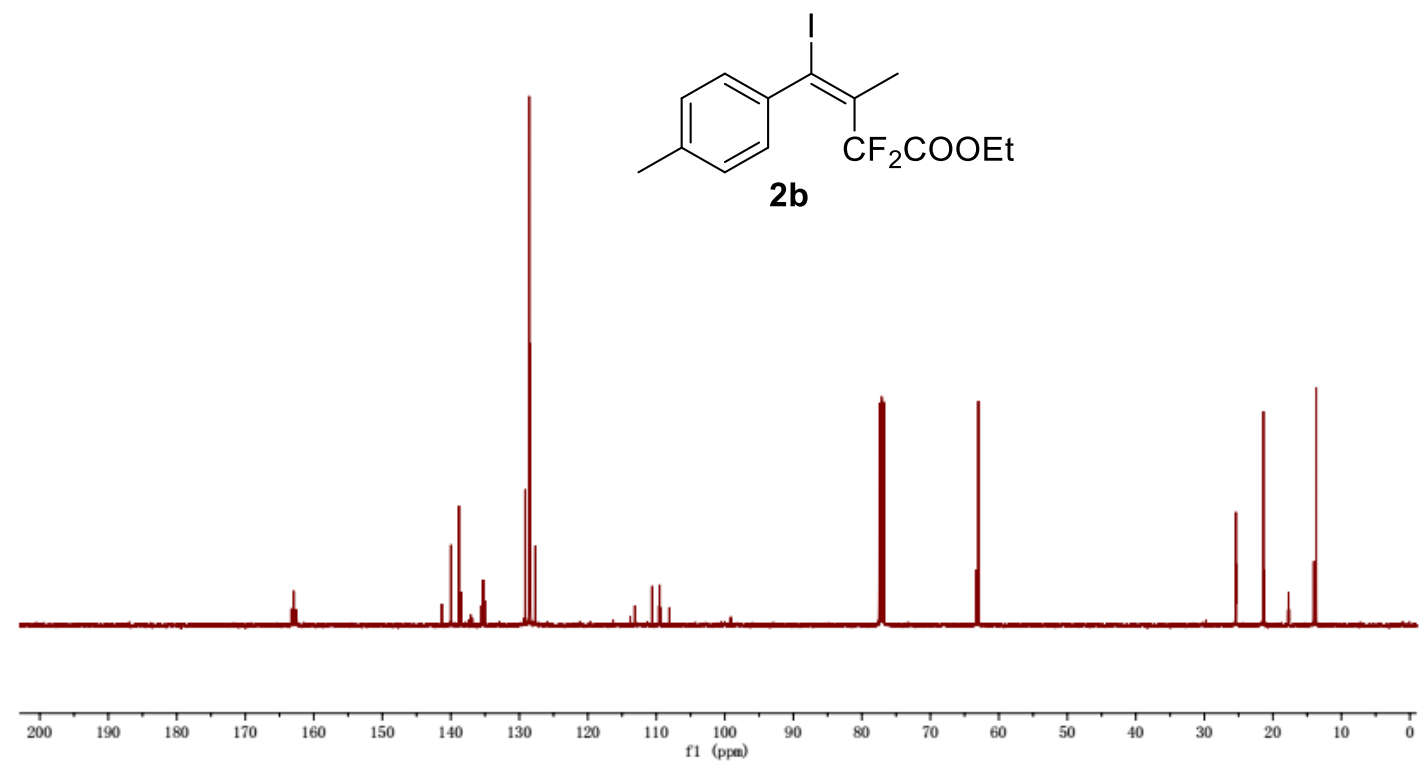


LKK-3-51-2
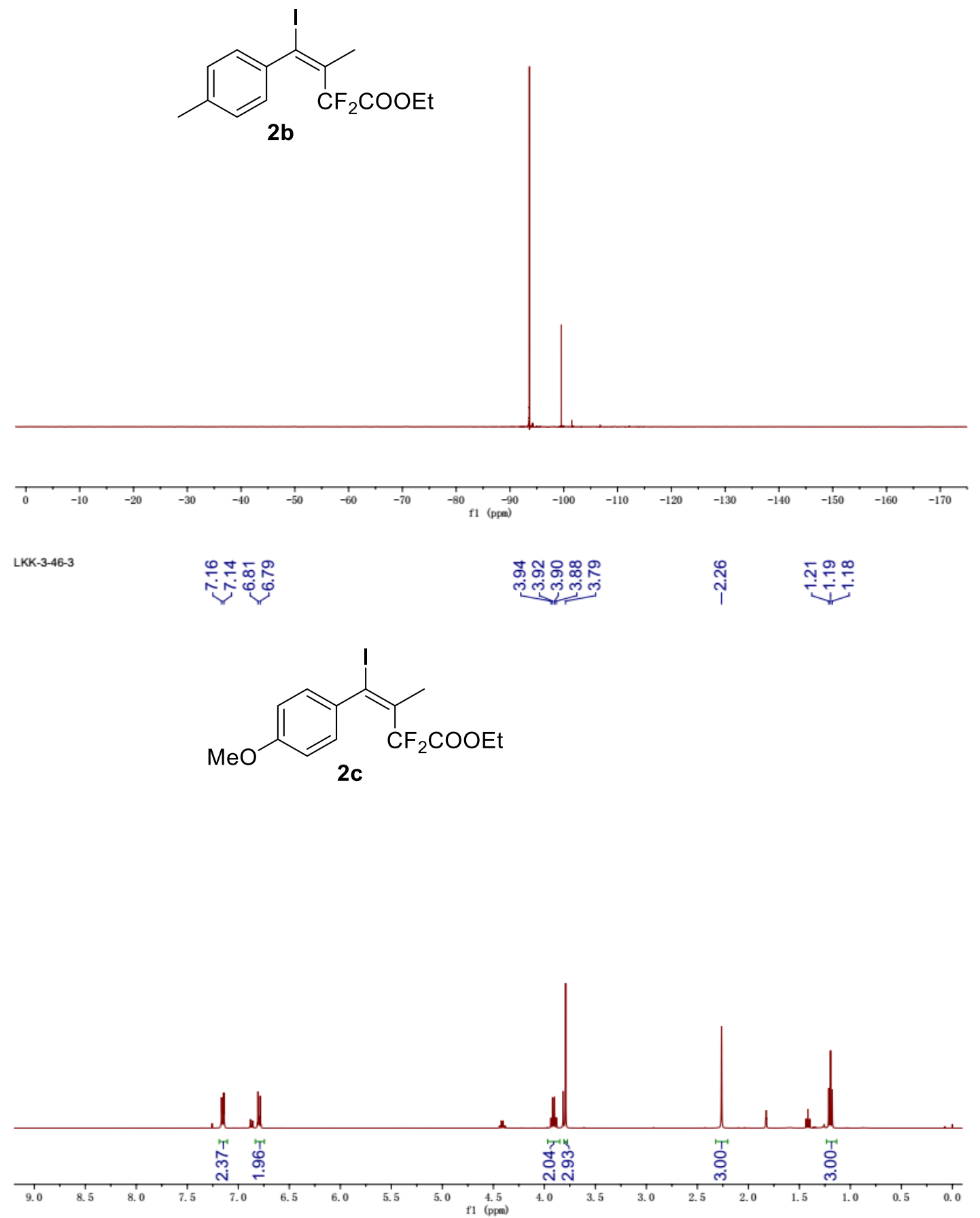

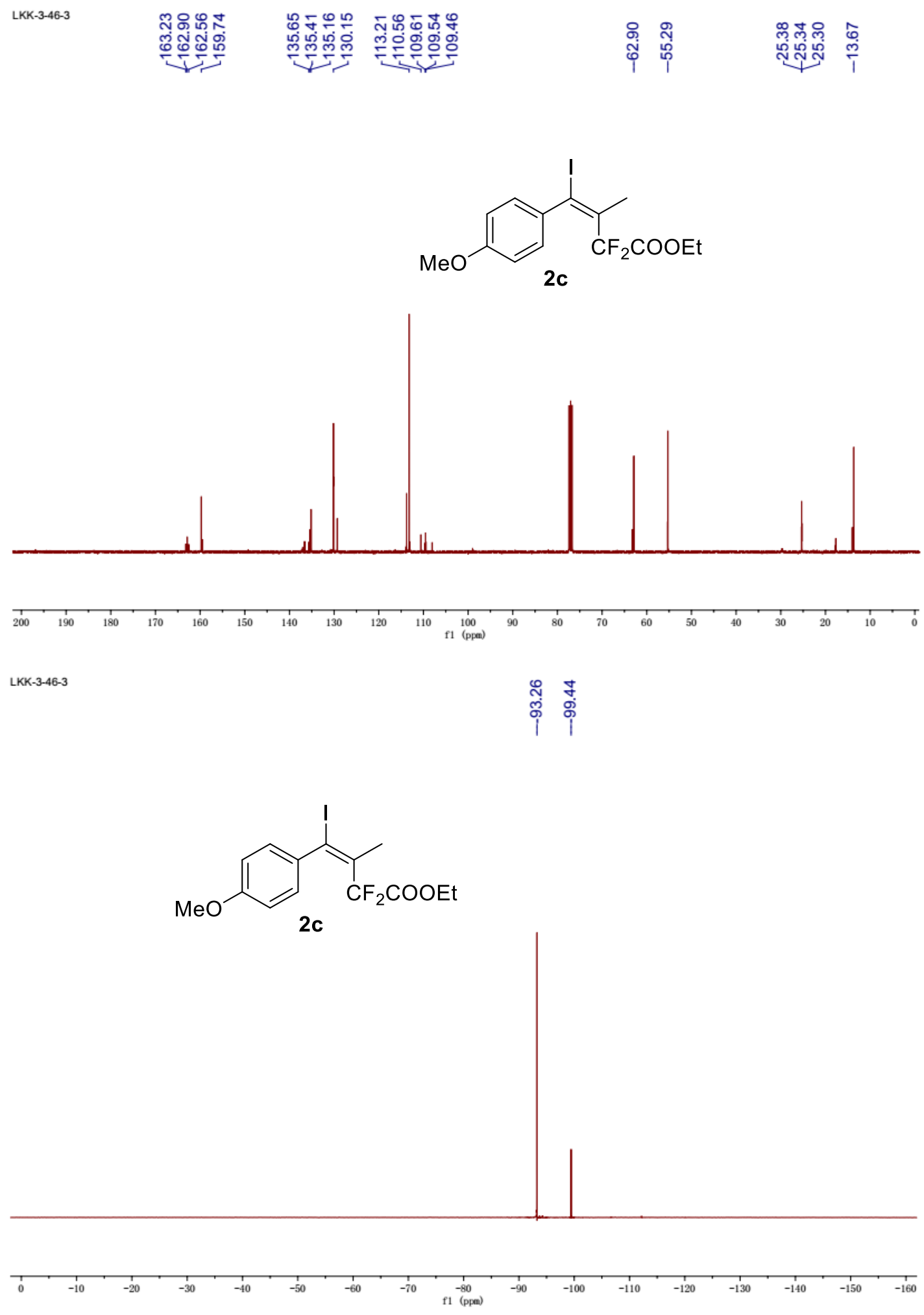


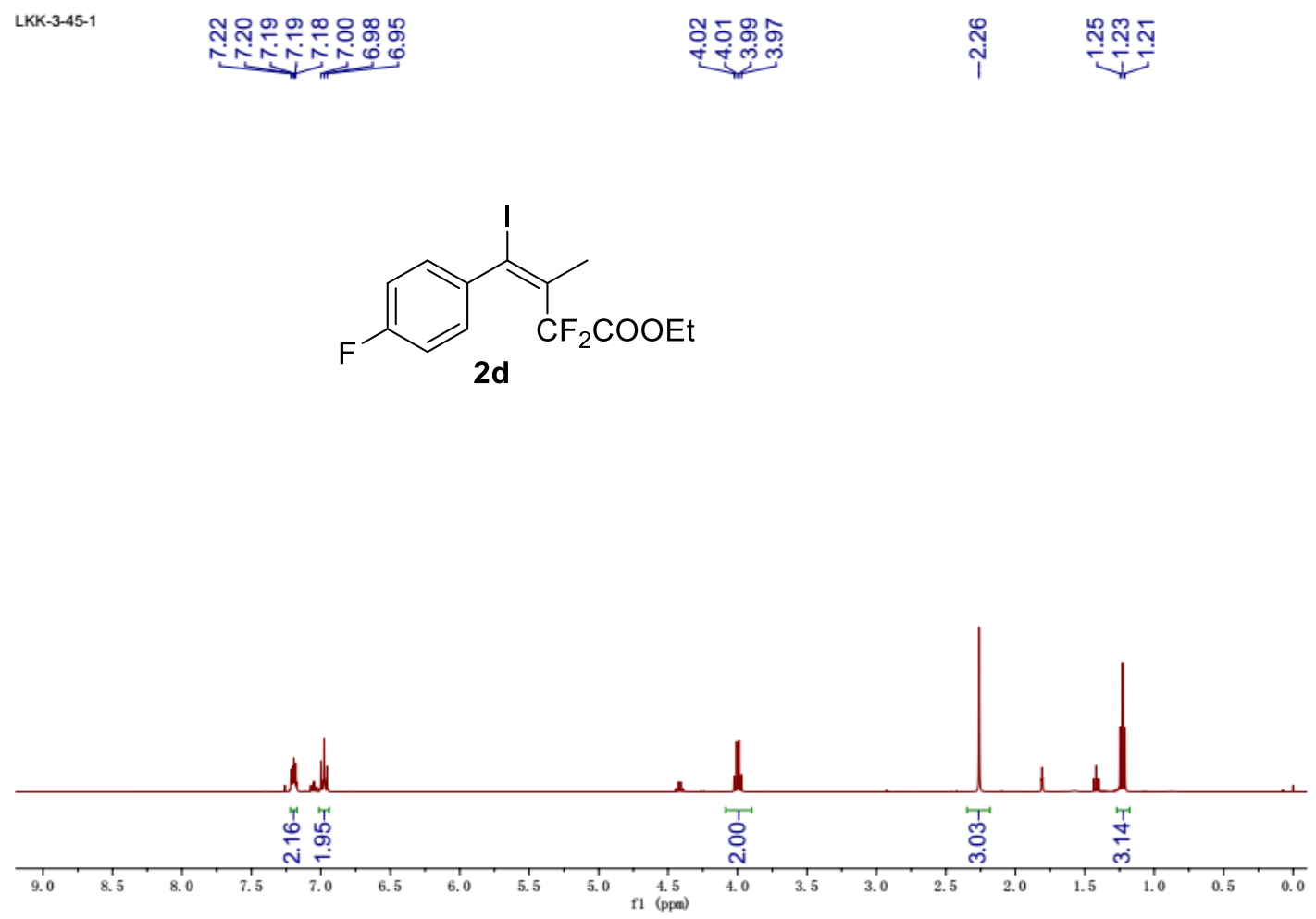

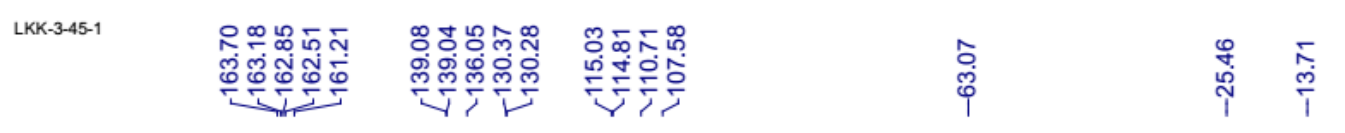
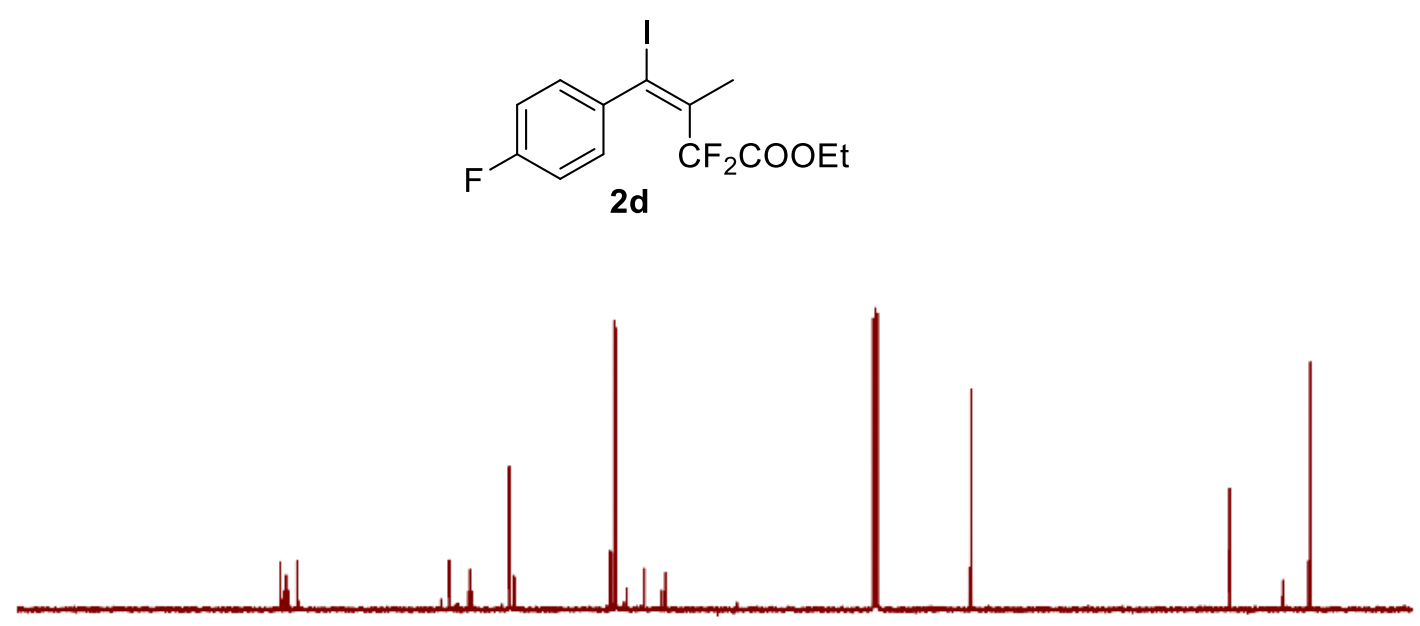

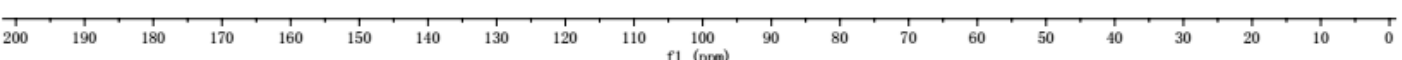


LKK-3-45-1

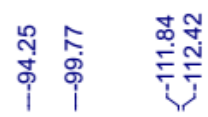
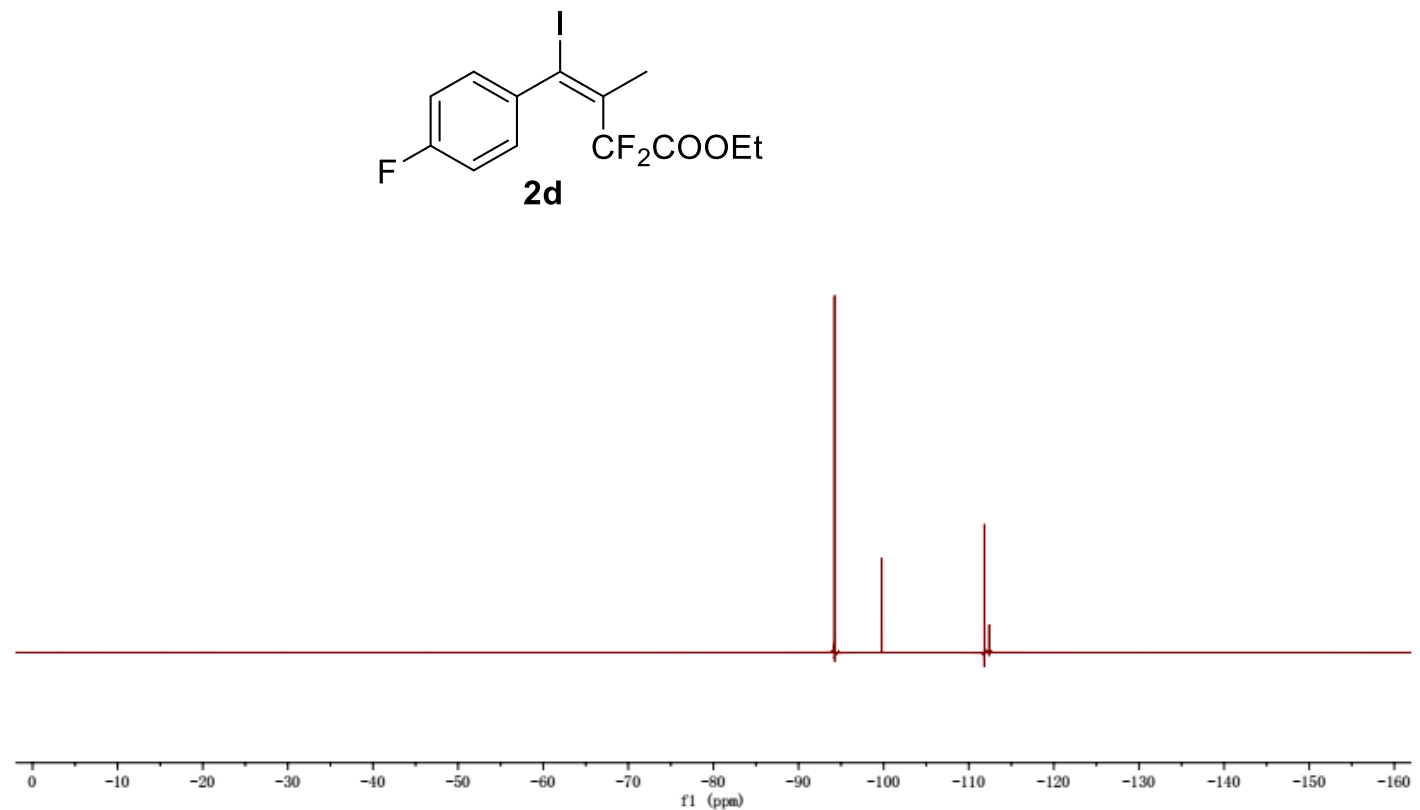

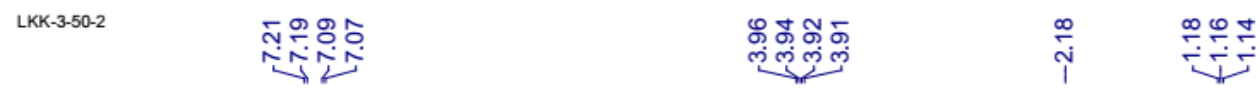
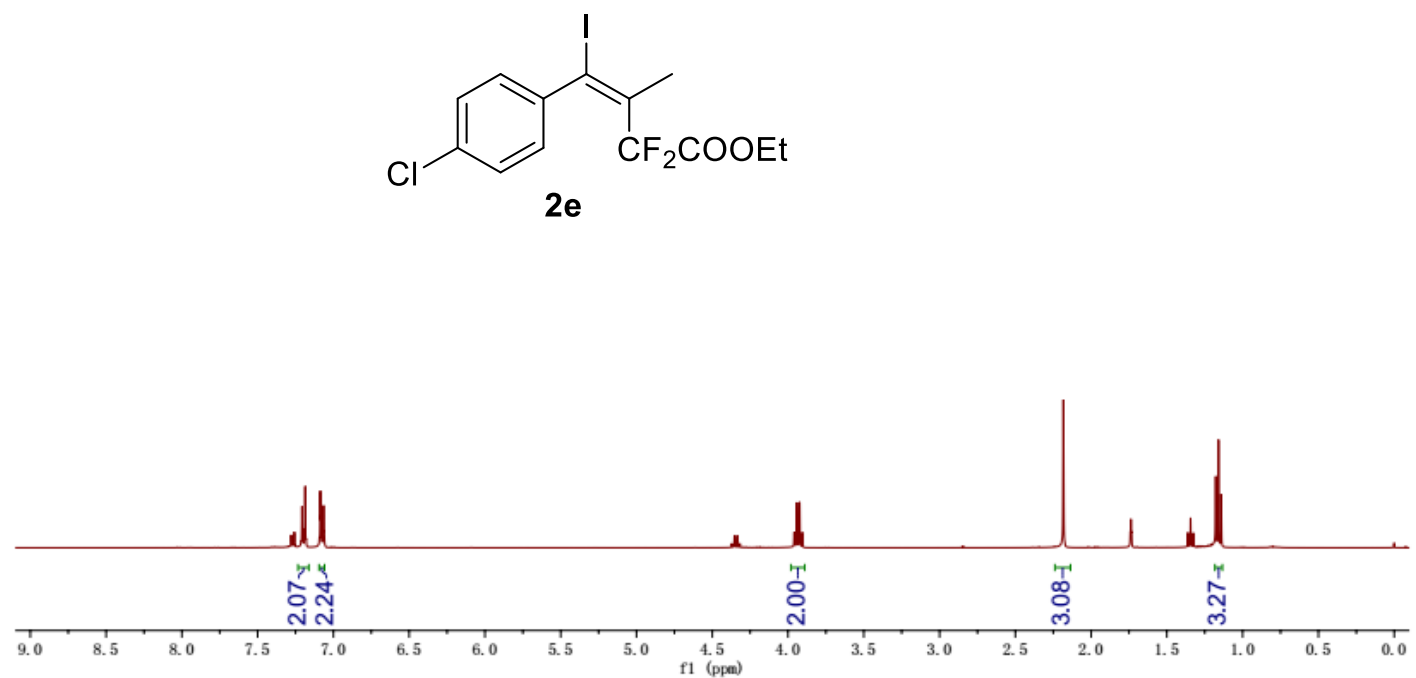


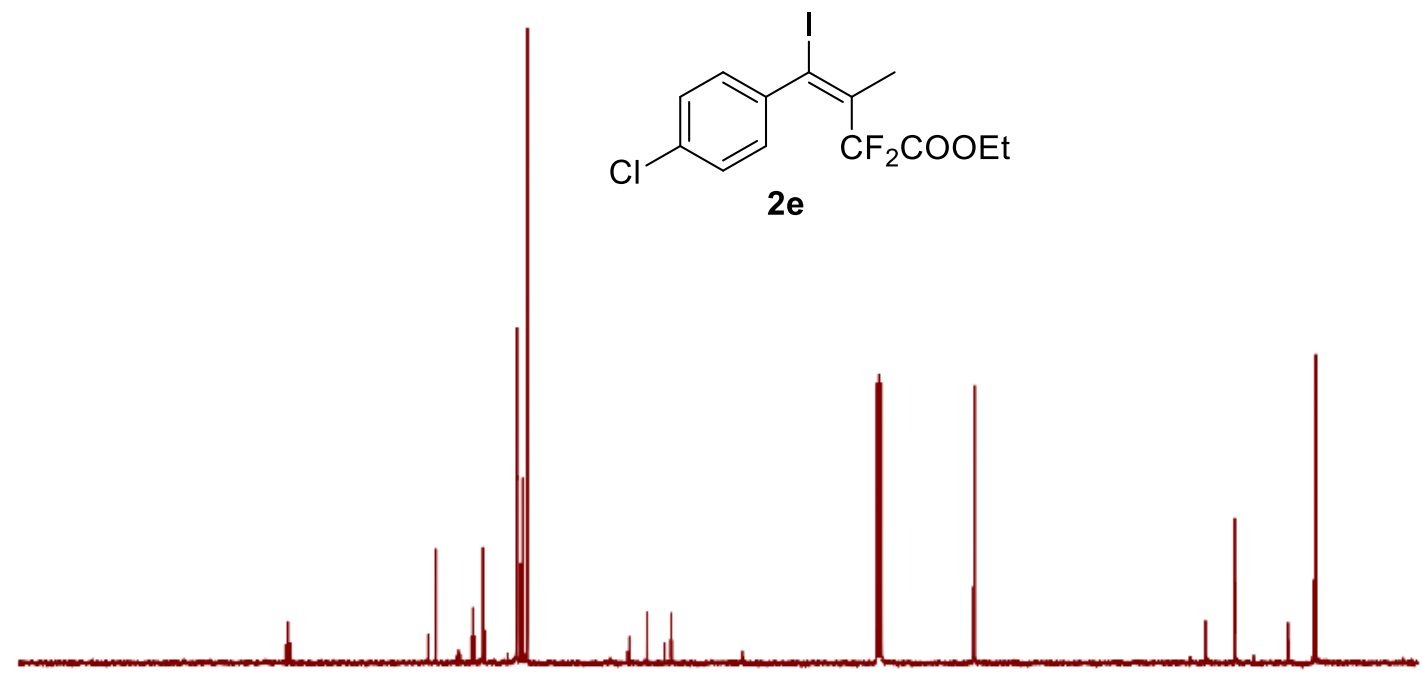$$
200
$$
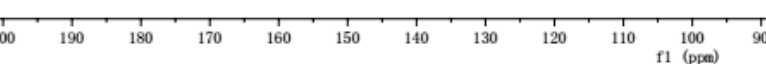

LKK-3-50-2

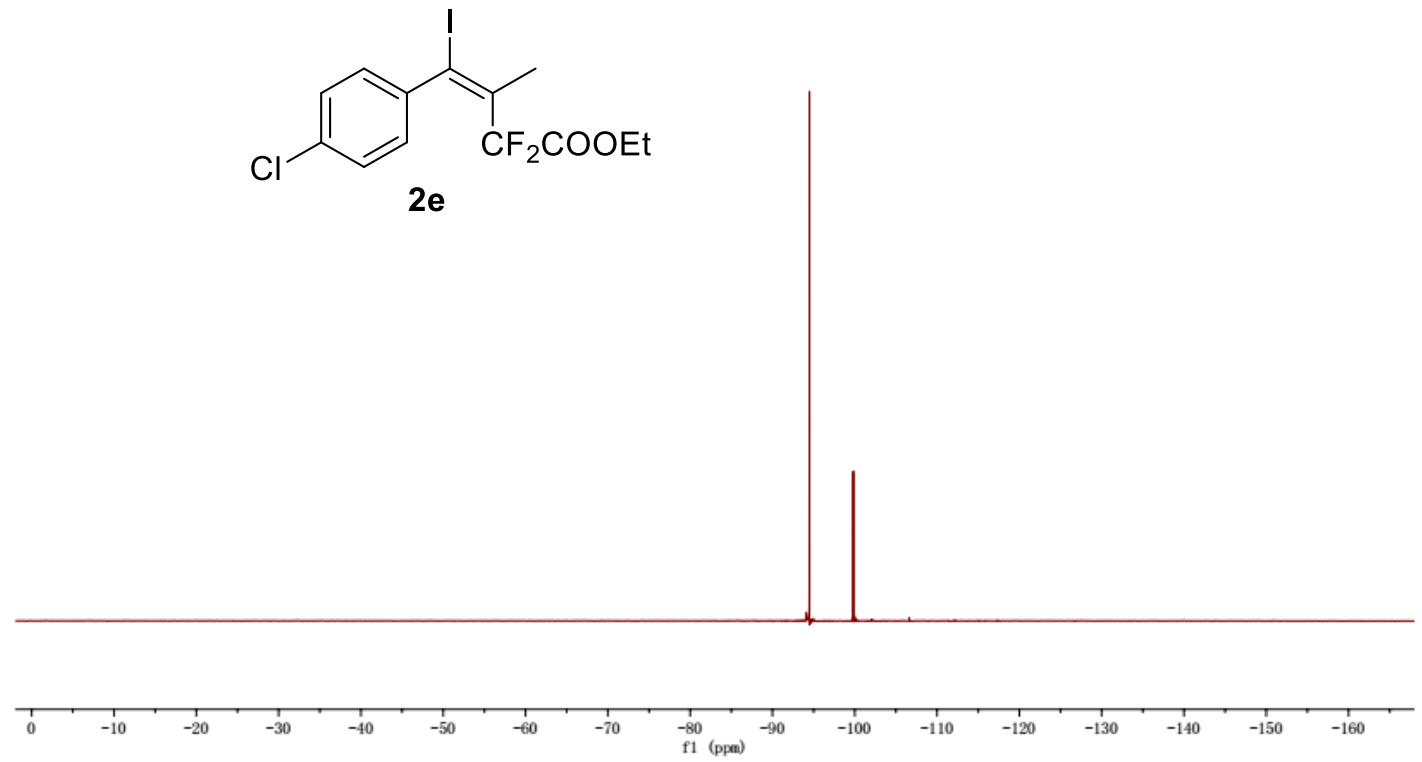



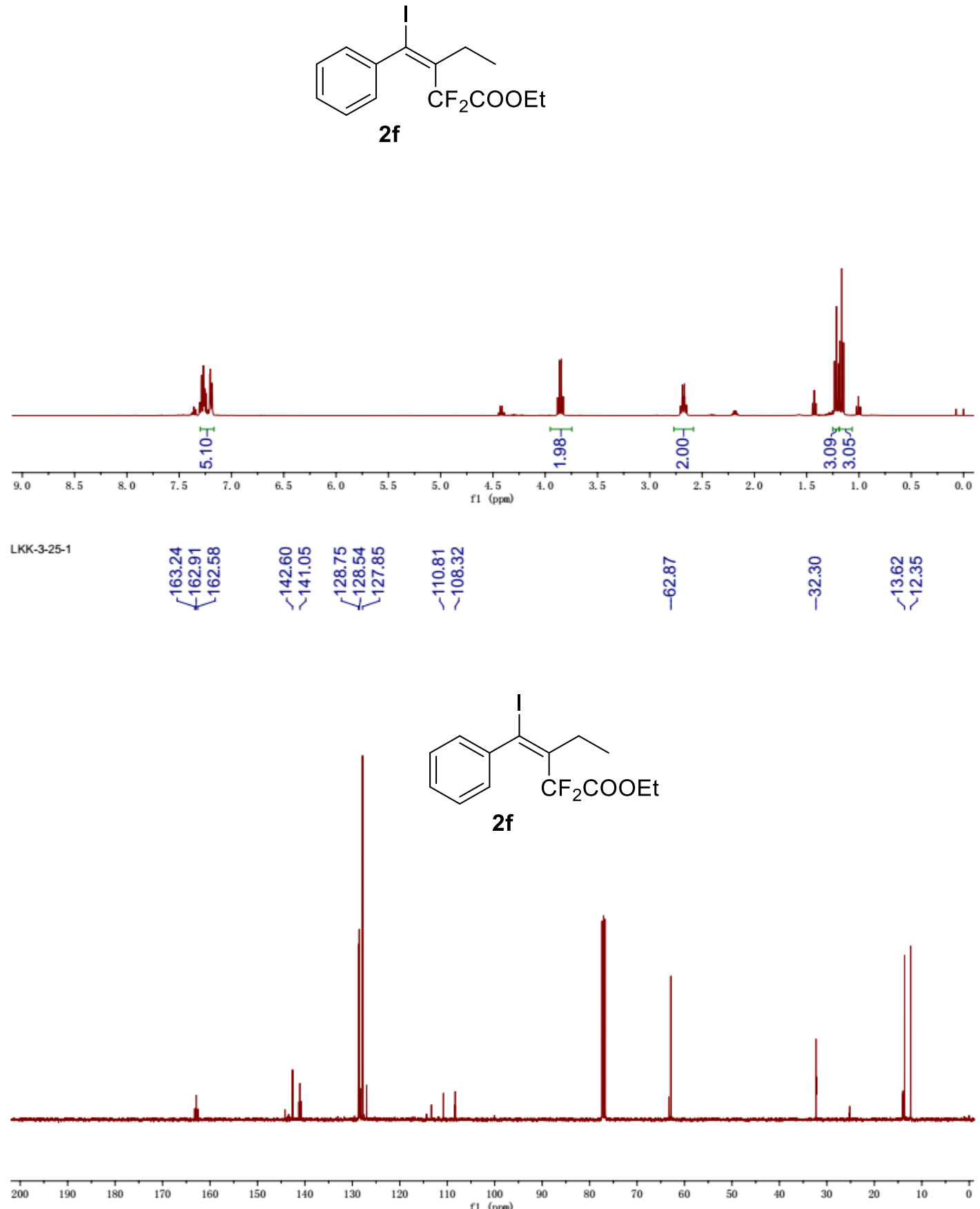


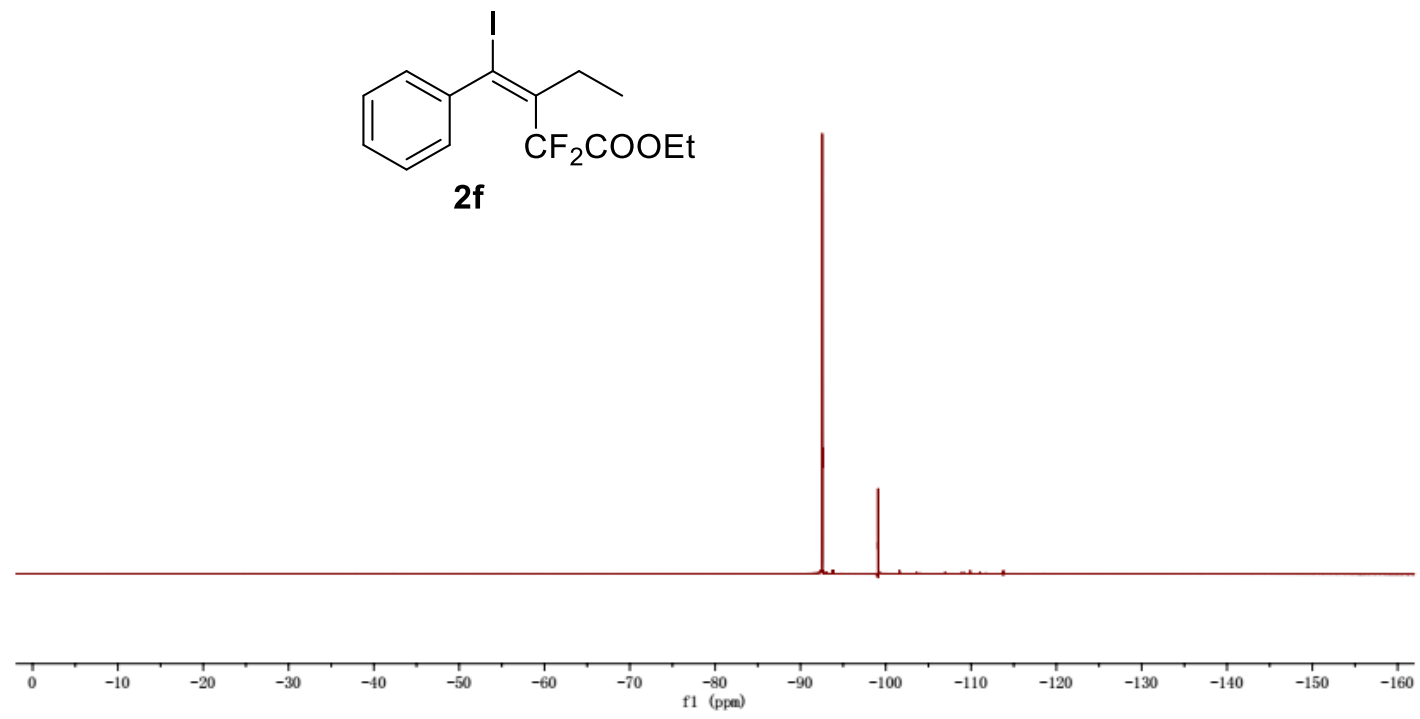

LKK-3-34-2

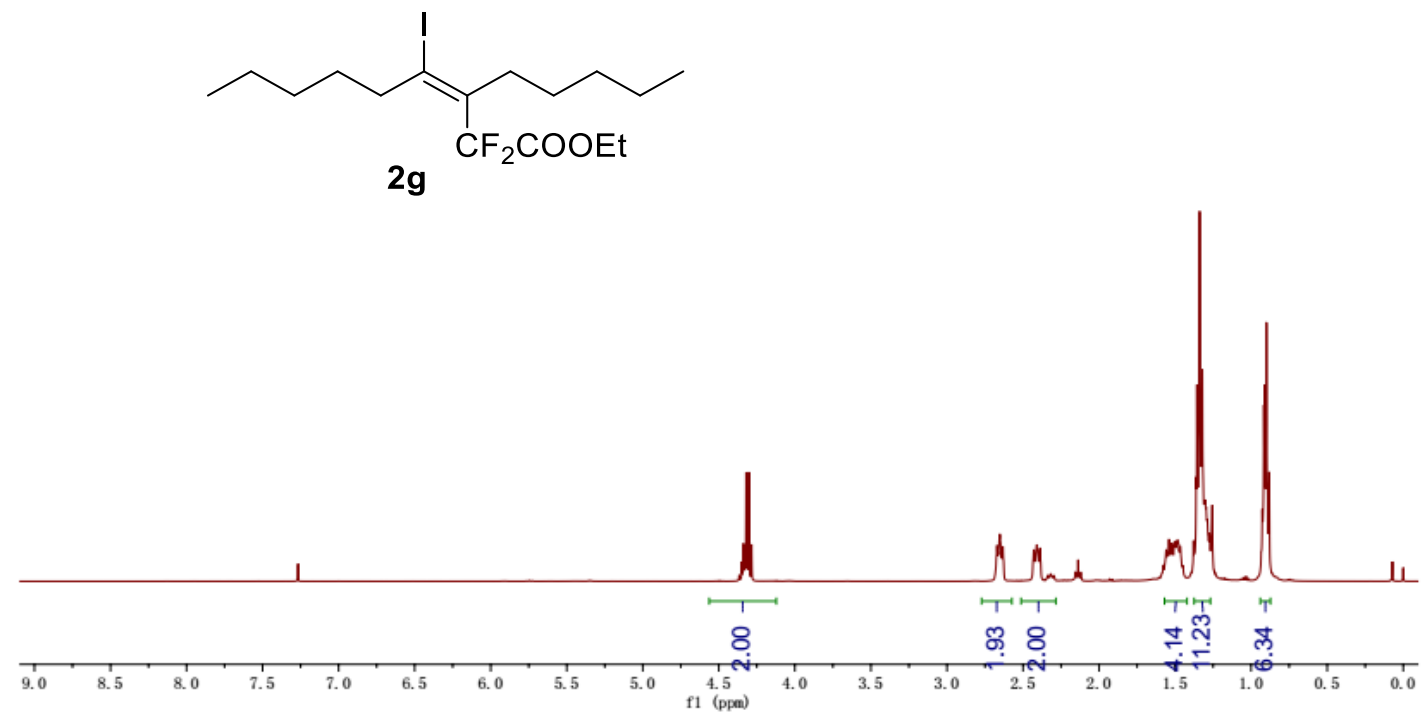


LKK-3-34-2

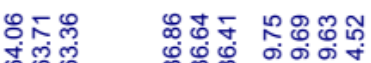

눈 管

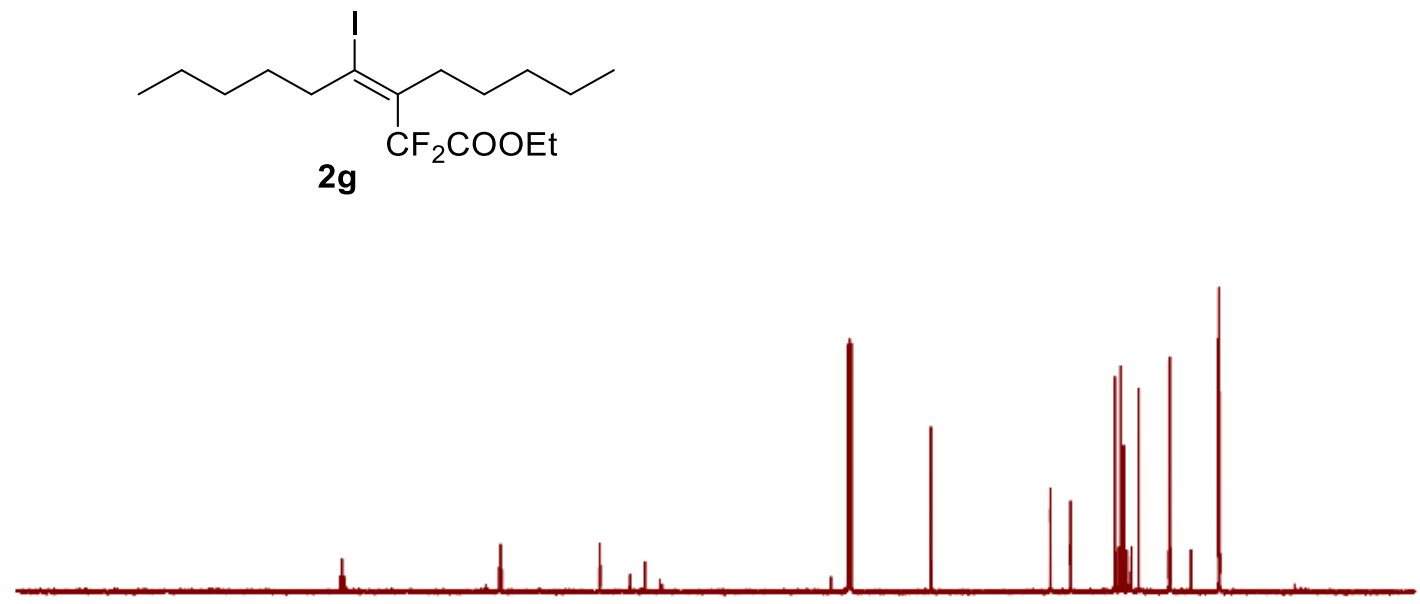

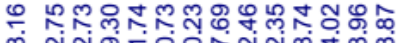

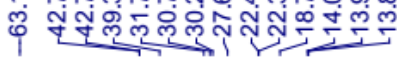

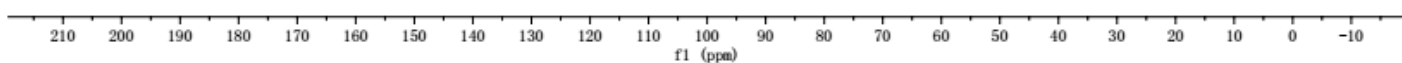

LKK-3-34-2
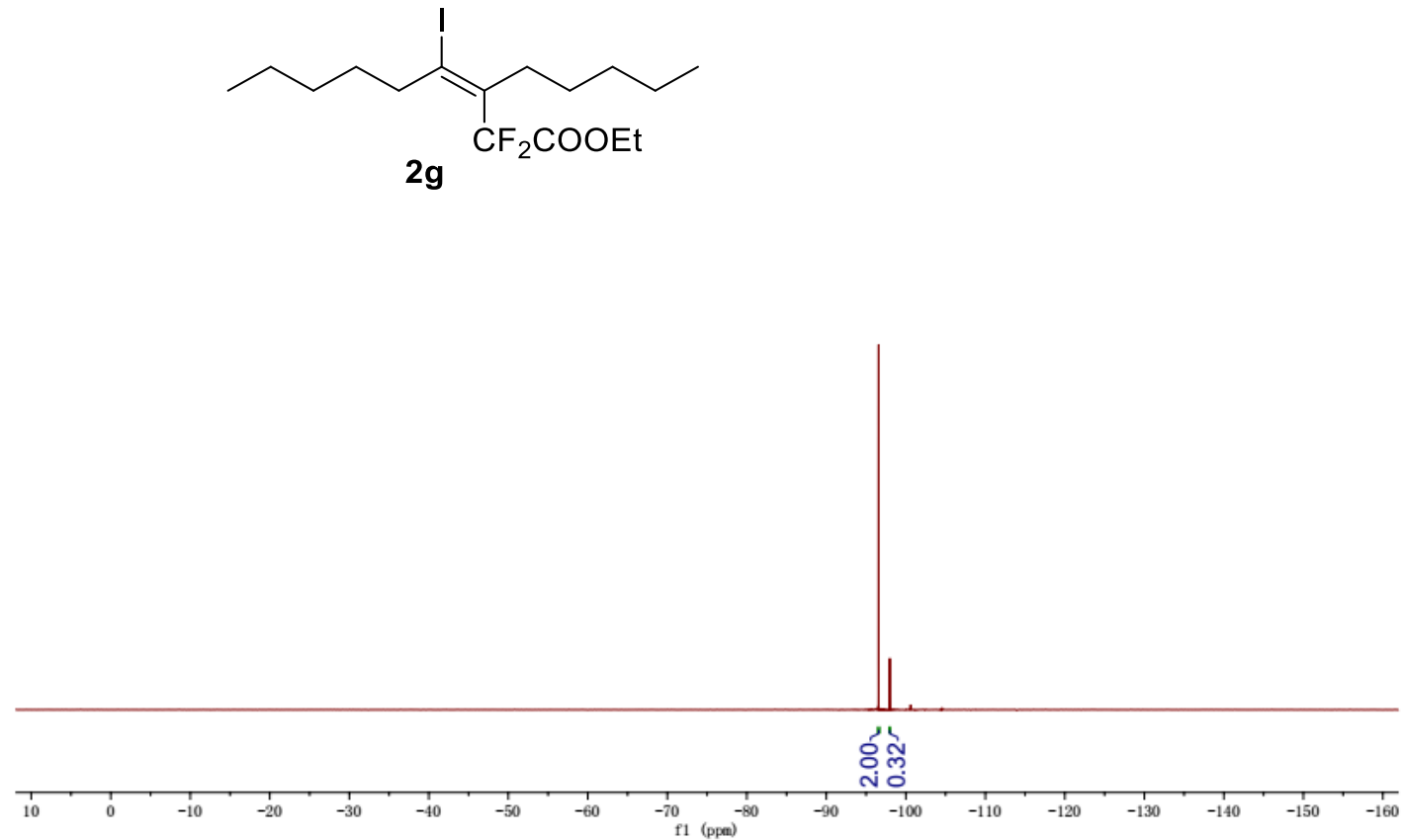


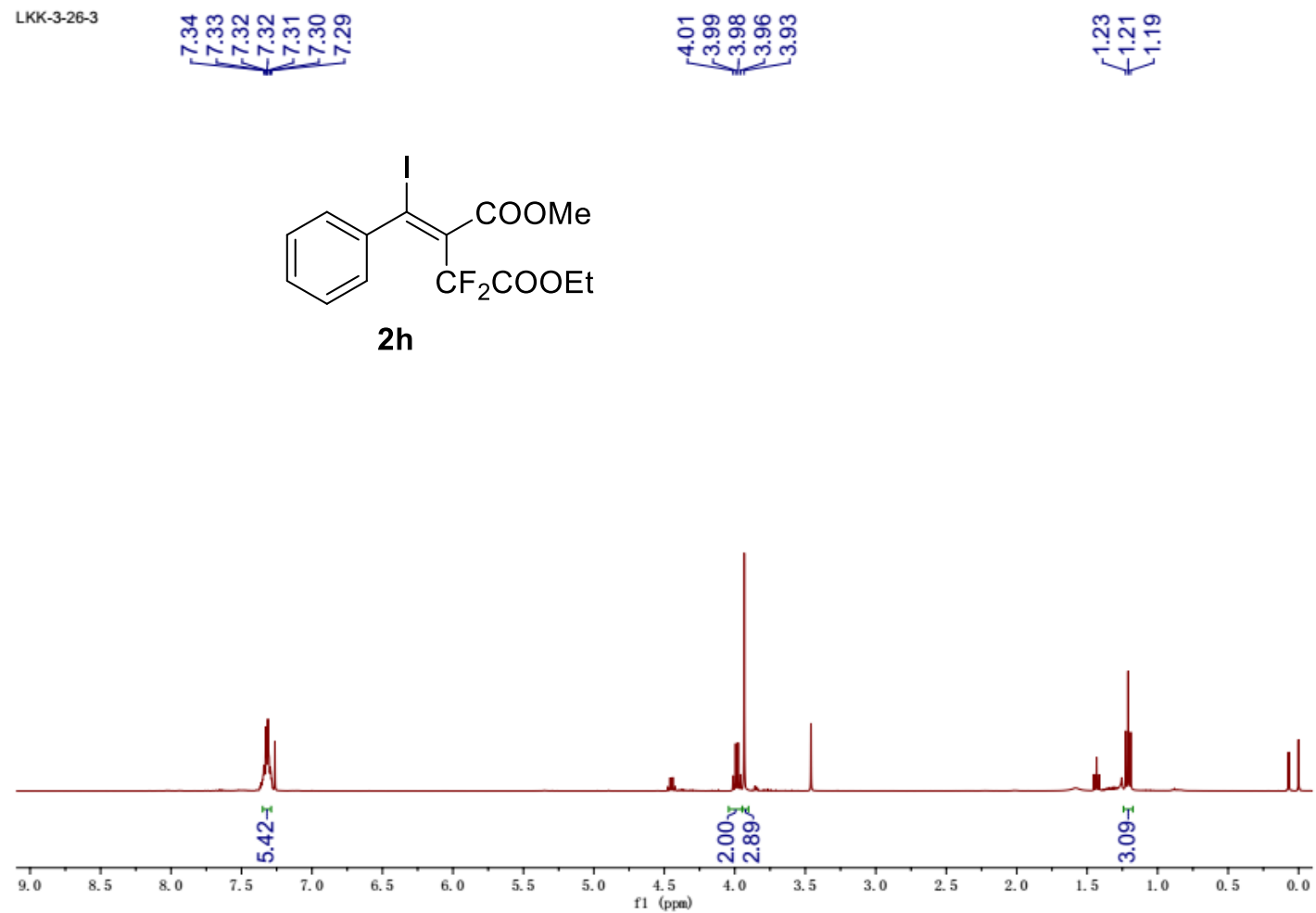

LKk-3-26-3

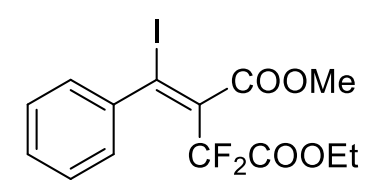

$2 \mathrm{~h}$

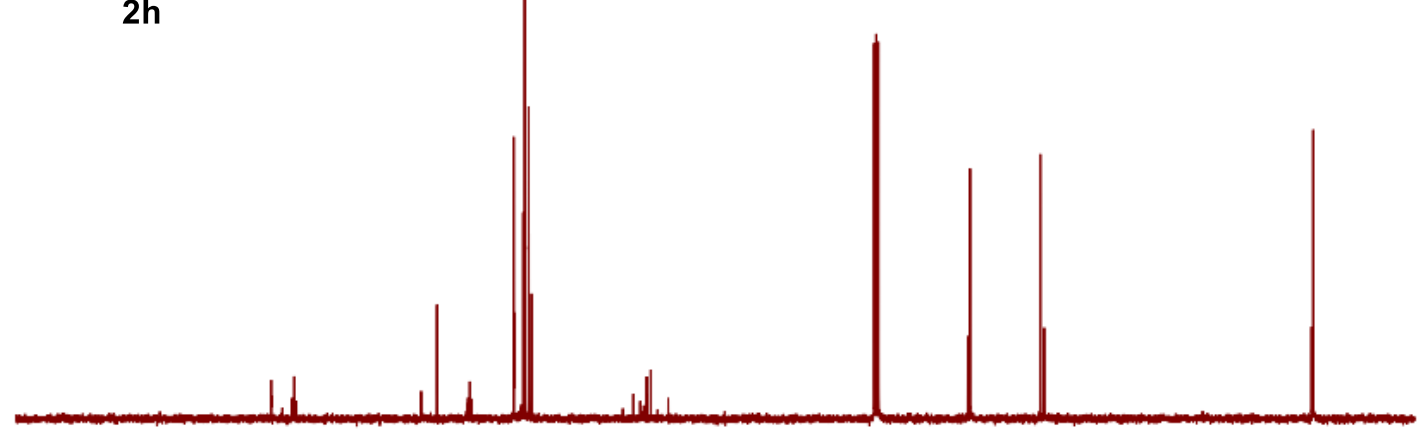

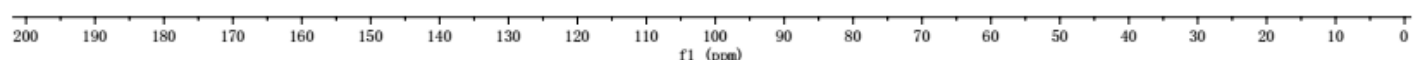


LKK-3-26-3
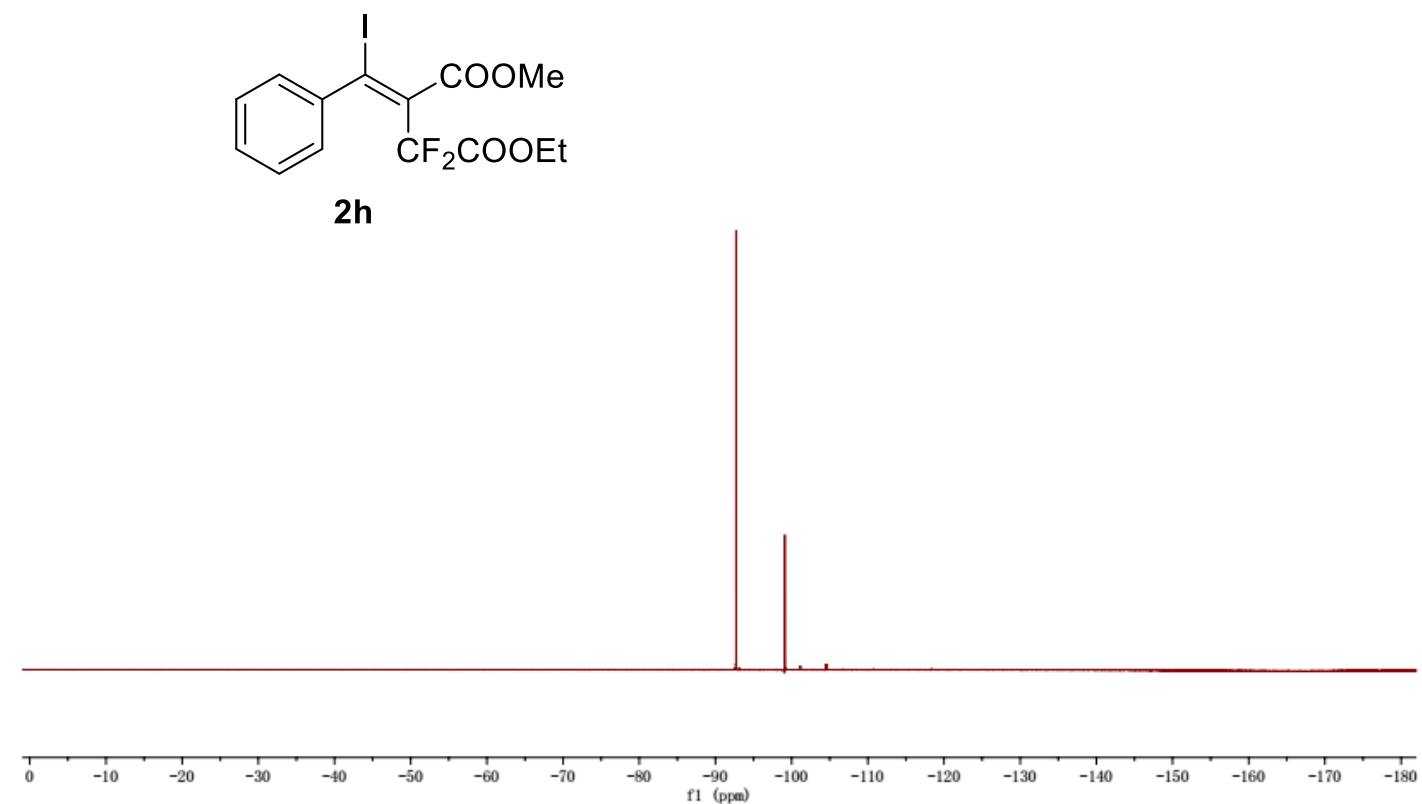

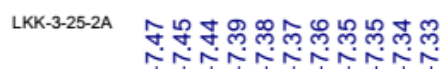

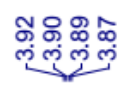

舟望<smiles>CCOC(=O)[C-](C(=C(I)c1ccccc1)c1ccccc1)c1ccccc1</smiles>

$2 i$

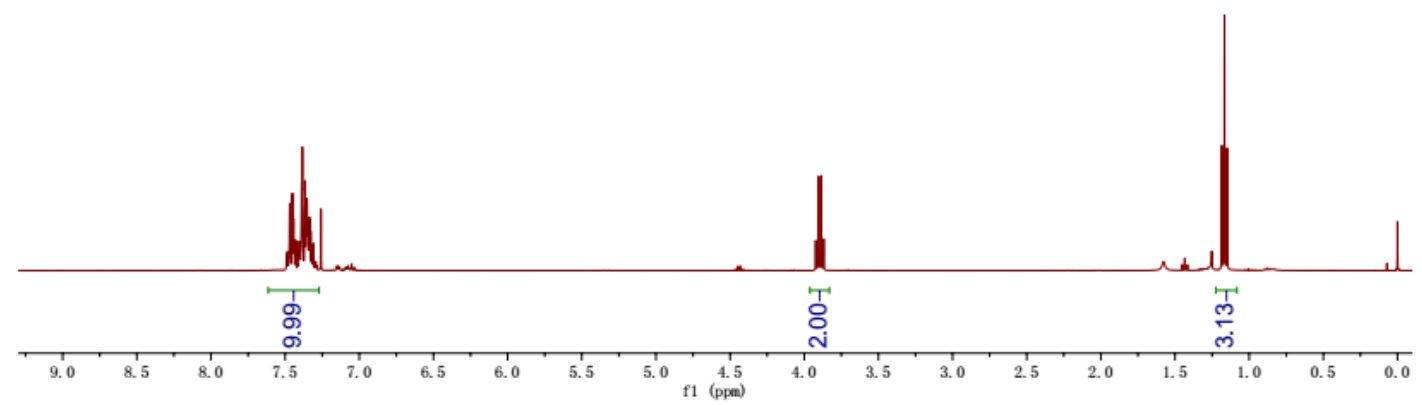




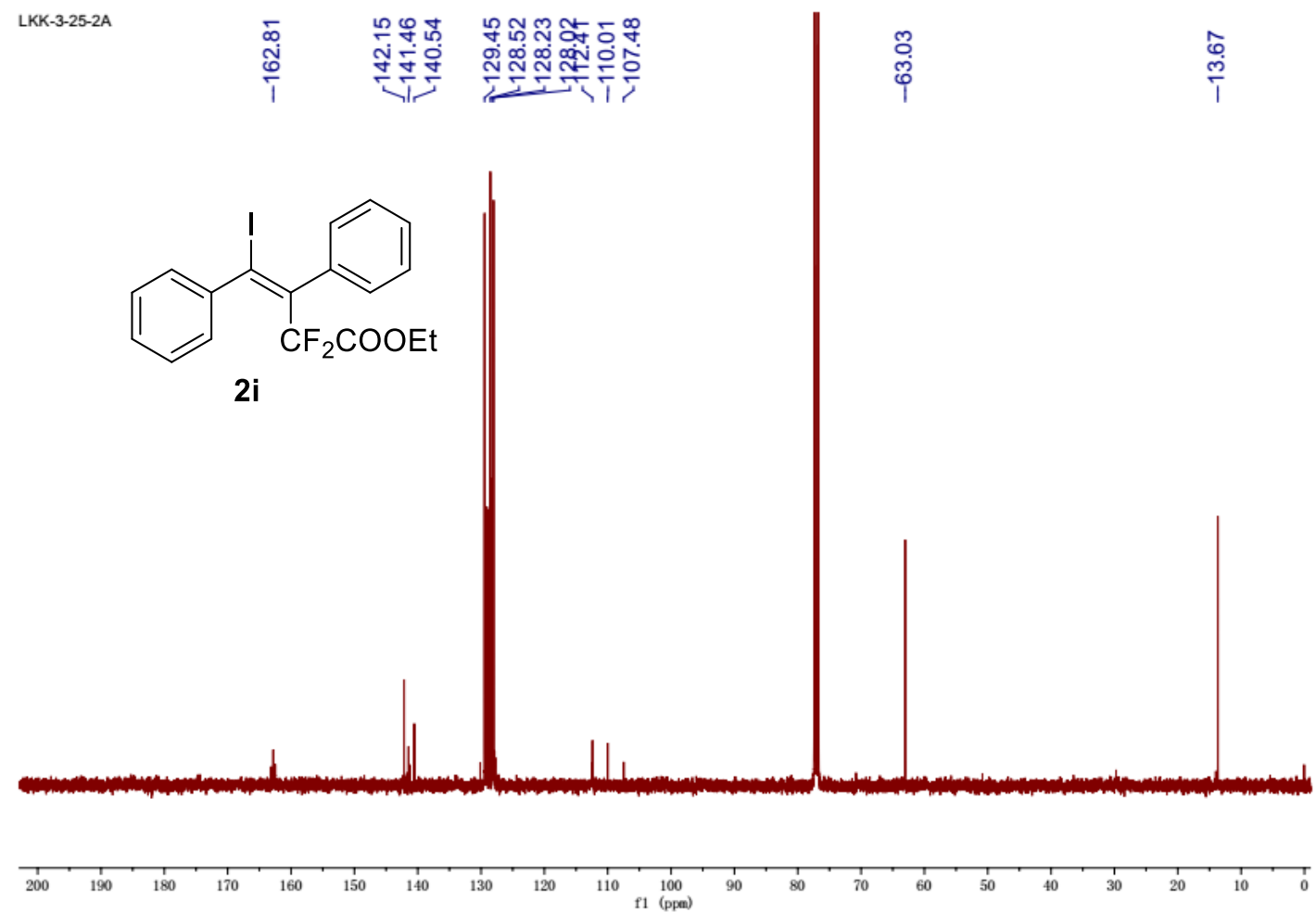

LKK-3-25-2A

管䔔<smiles>CCOC(=O)C(=C(C(=C(I)c1ccccc1)c1ccccc1)C(F)(F)F)c1ccccc1</smiles>

2i

${ }_{0}^{1}-10 \quad-20$

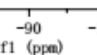



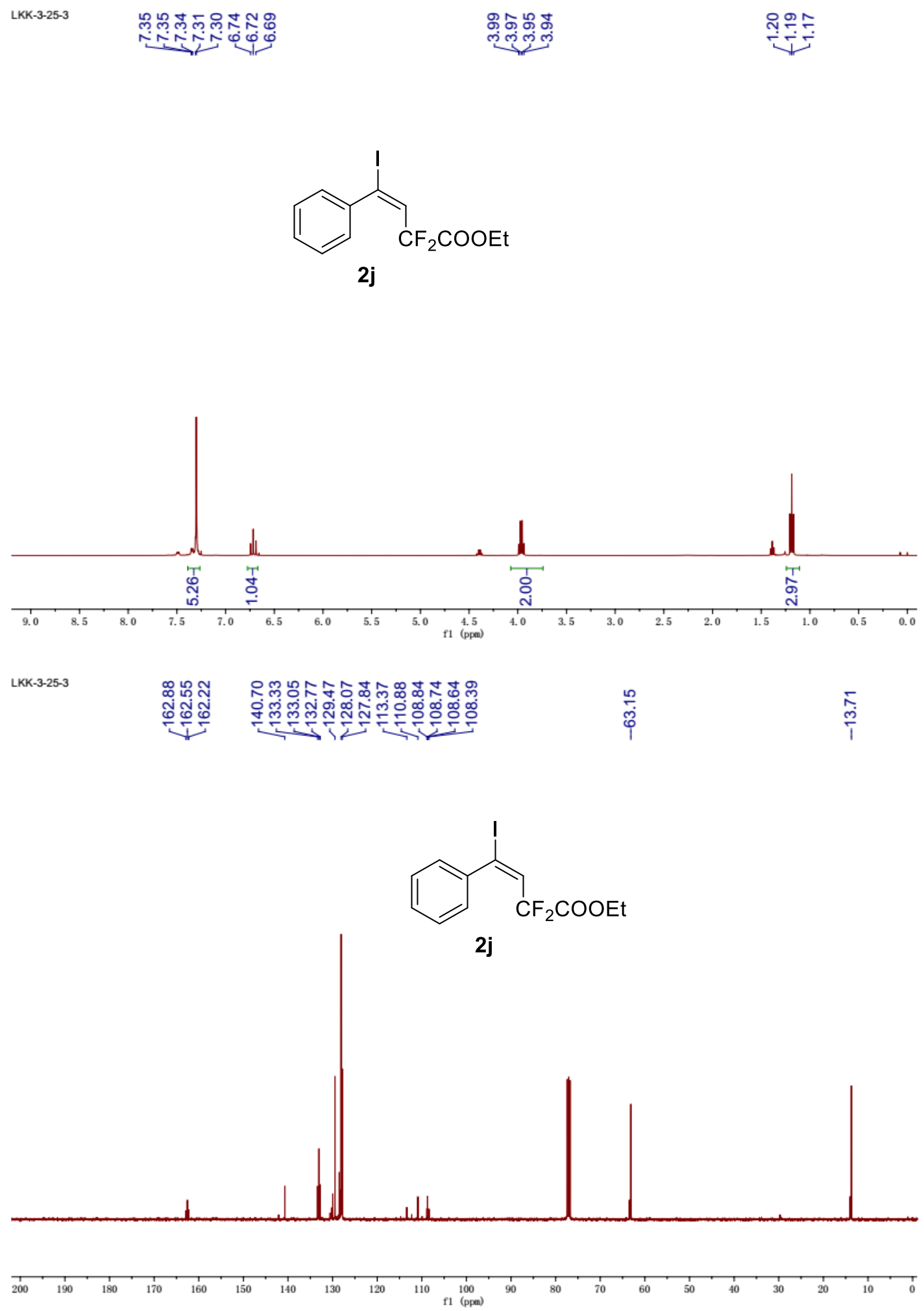

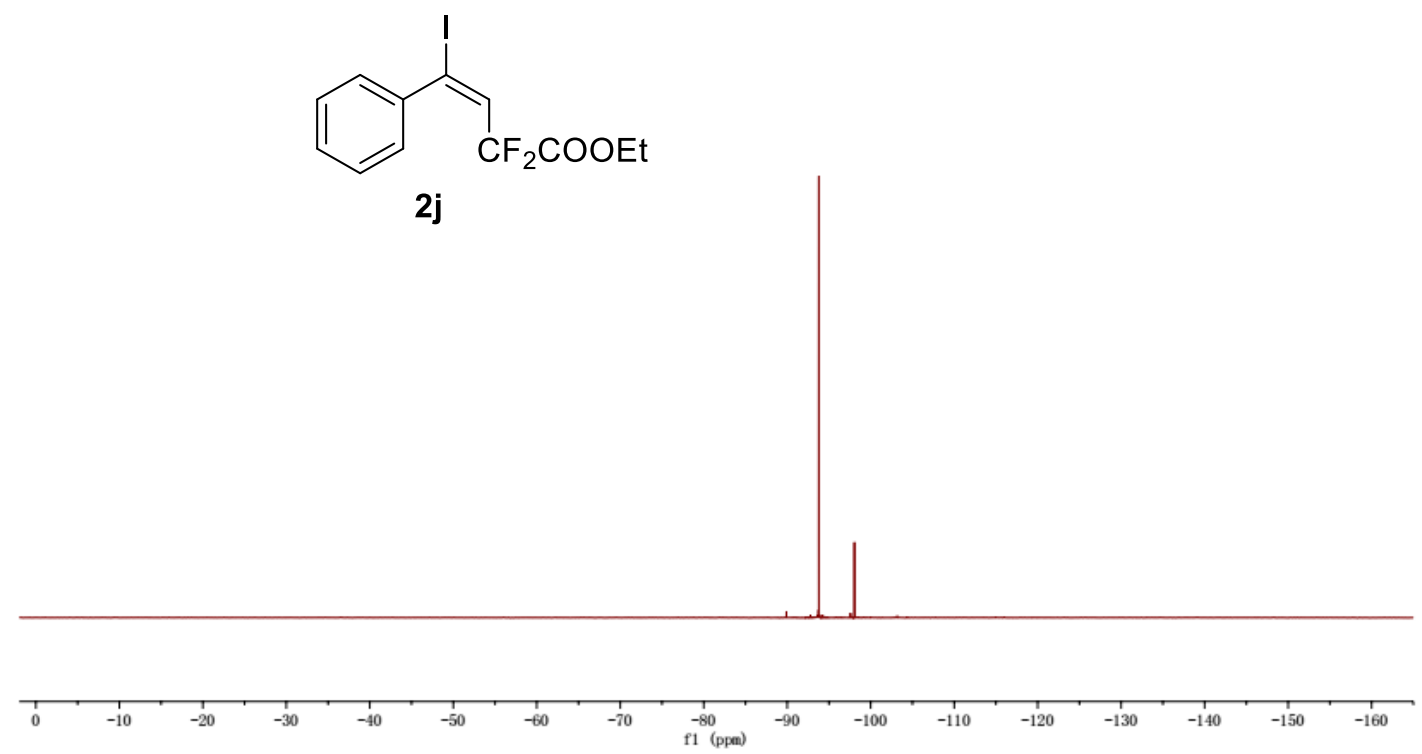

LKK-3-29-3

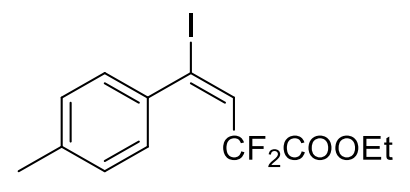

2k

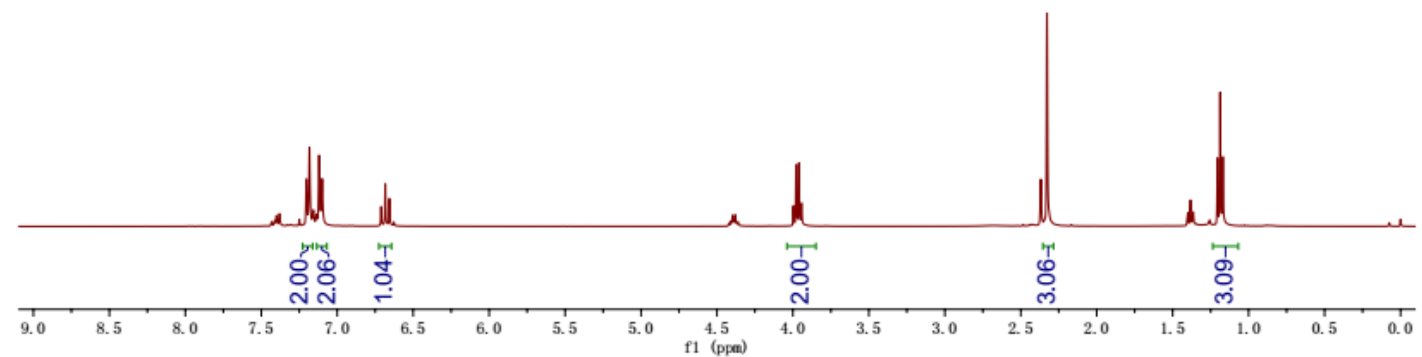


LKK-3-29-3
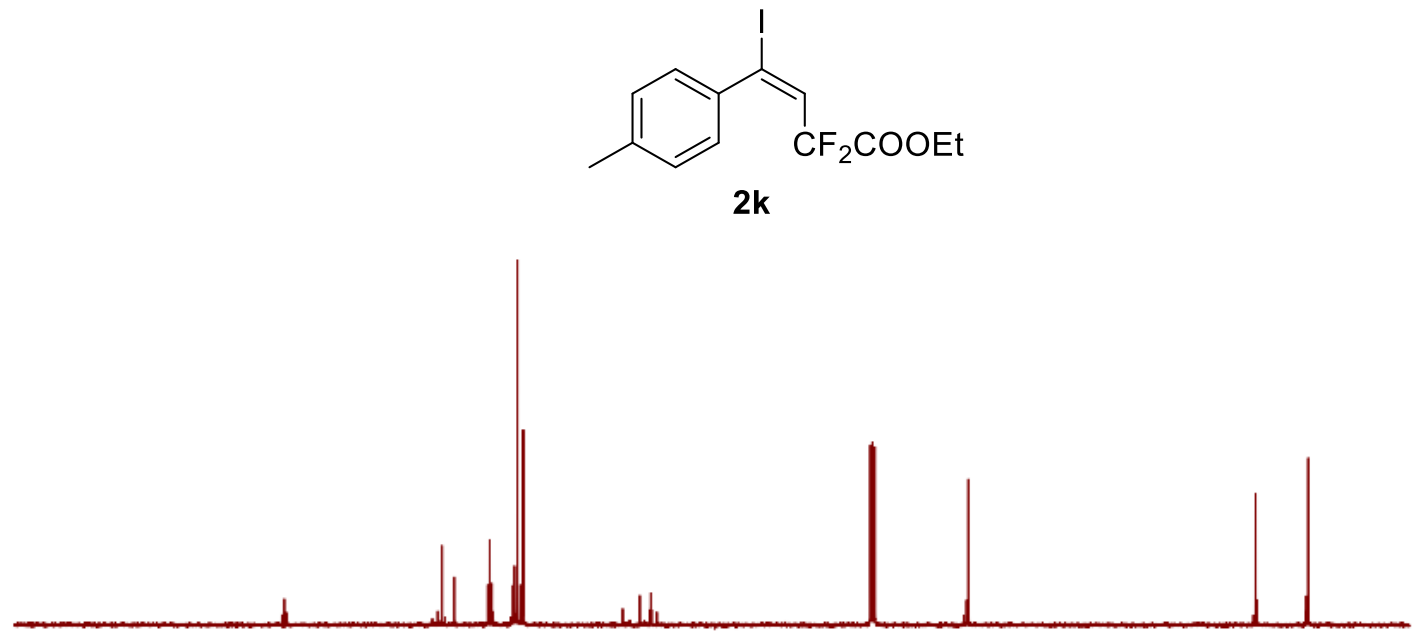

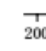

-

LKK-3-29-3

:

क् के<smiles>CCOC(=O)C(F)(F)/C=C(/I)c1ccc(C)cc1</smiles>

2k

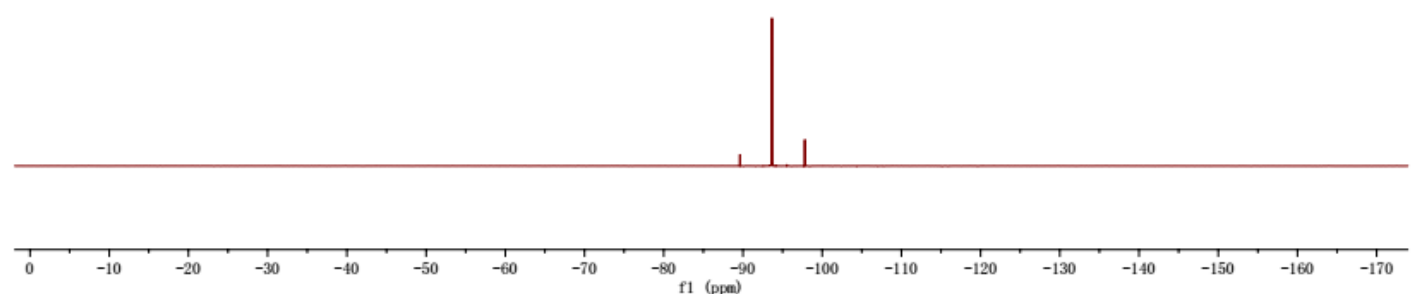


<smiles>CCOC(=O)[C-](C)/C=C(/I)c1cccc(C)c1</smiles>

21

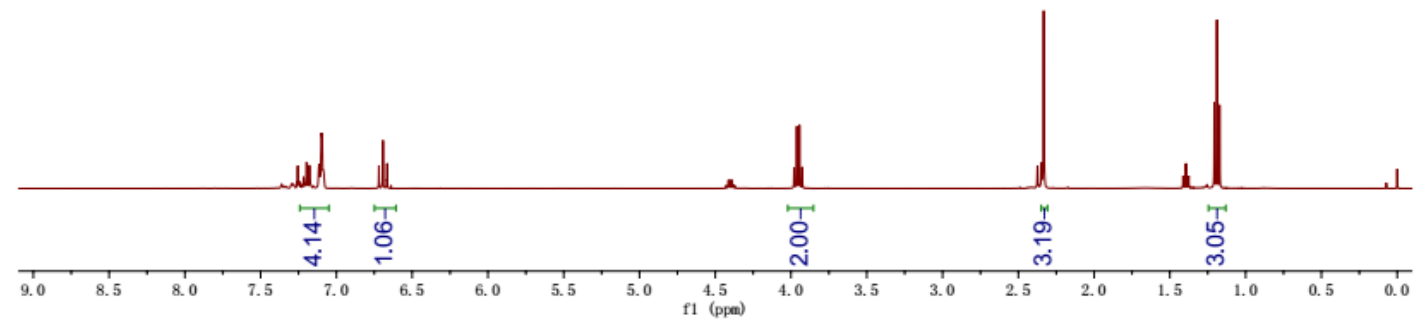

LKK-3-32-3

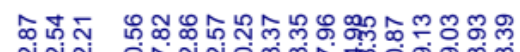

ஸ่ํำ

is

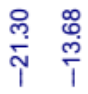<smiles>CCOC(=O)C(=CC(I)c1cccc(C)c1)C(=O)OCC</smiles>

2I

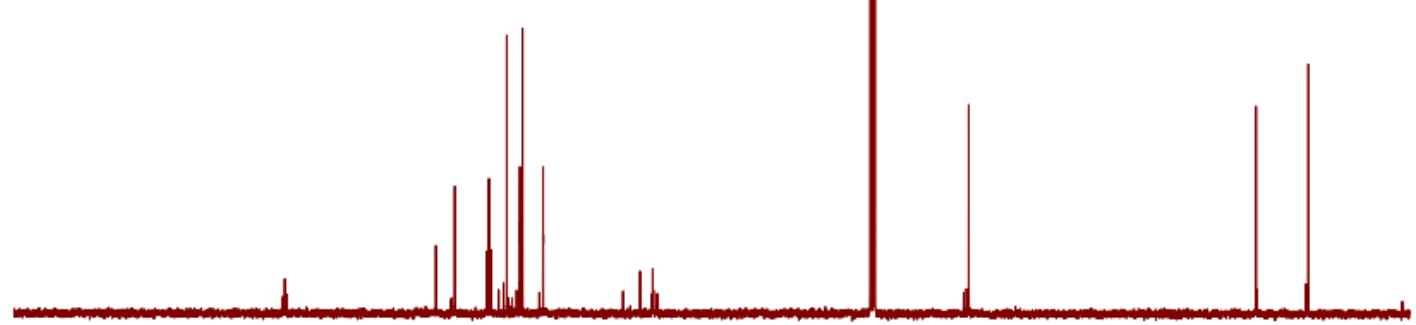

$\frac{1}{200}$

190

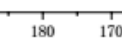

${ }_{160}^{1}$

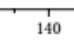

$10 \quad \stackrel{100}{\mathrm{f} 1}(\mathrm{ppm})$ 


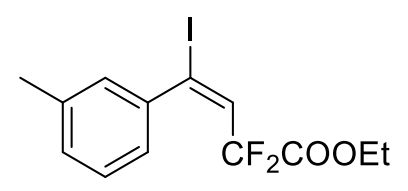

21
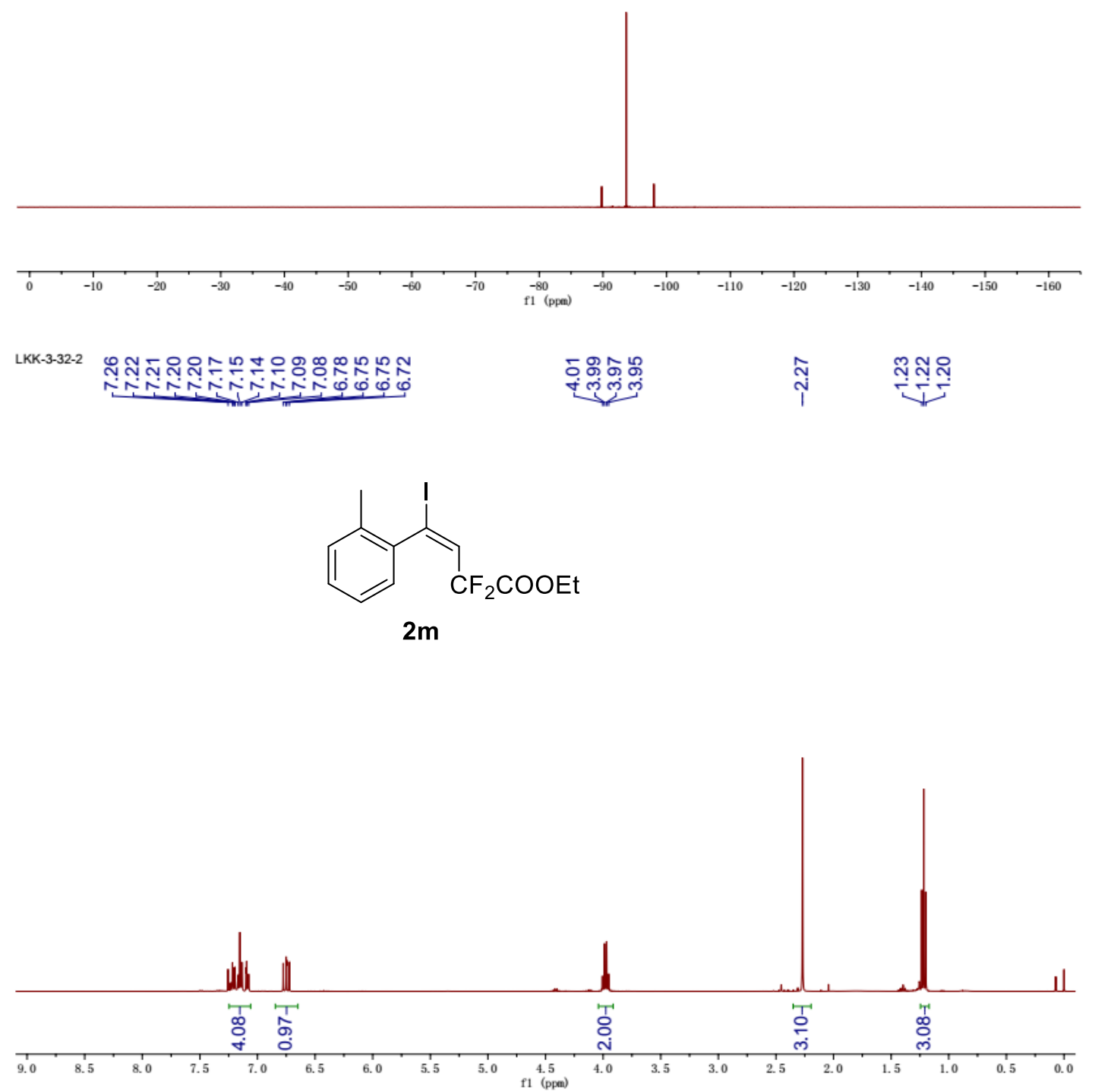
<smiles>CCOC(=O)C(F)(F)C=C(I)c1ccccc1C</smiles>

$2 \mathrm{~m}$

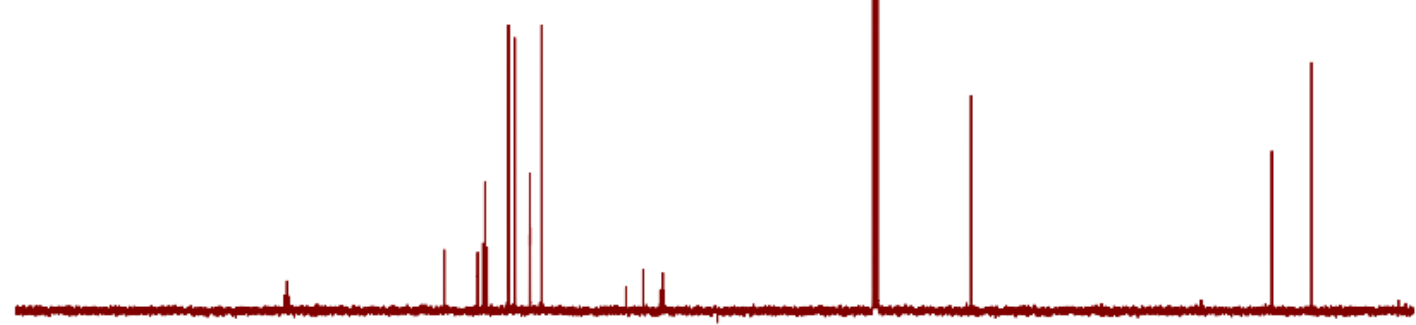

\section{$=$}

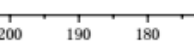

LKK-3-32-2

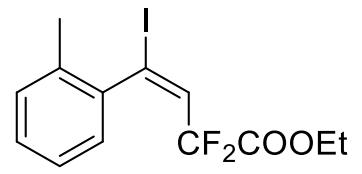

$2 m$
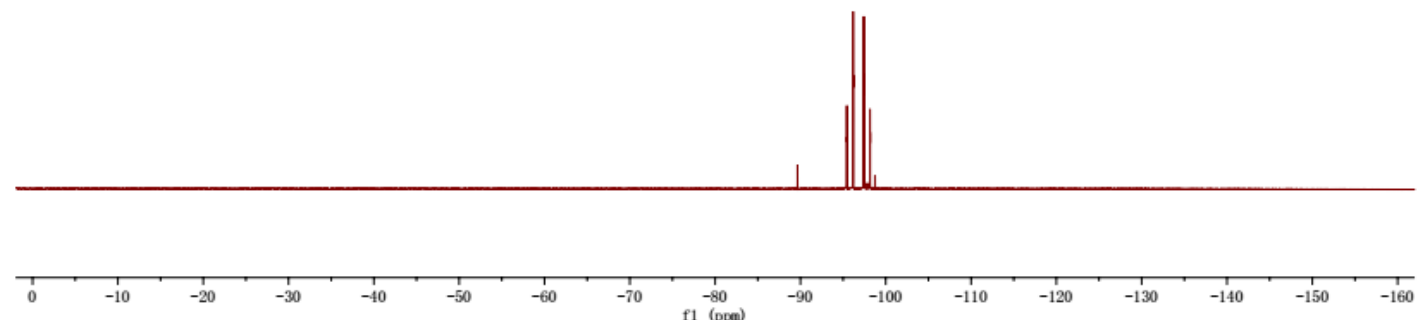
LKK-3-26-2

ธ요용

ำ ำ

范

लुल लं

ำ
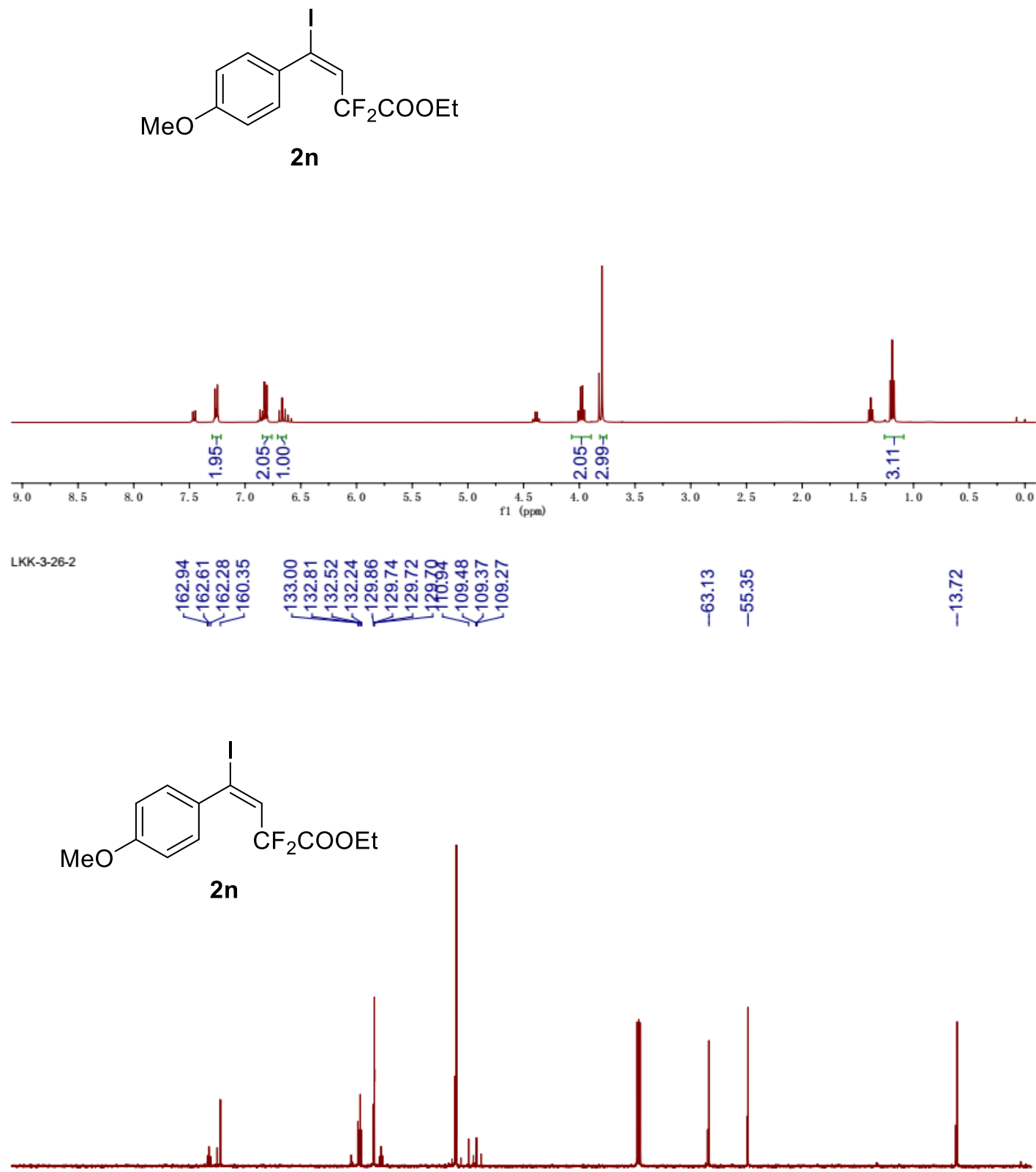

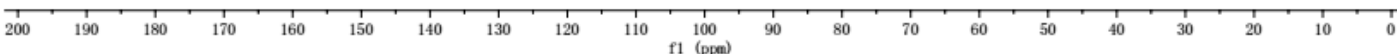


LKK-3-26-2

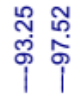<smiles>CCOC(=O)C(F)(F)/C=C(/I)c1ccc(OC)cc1</smiles>
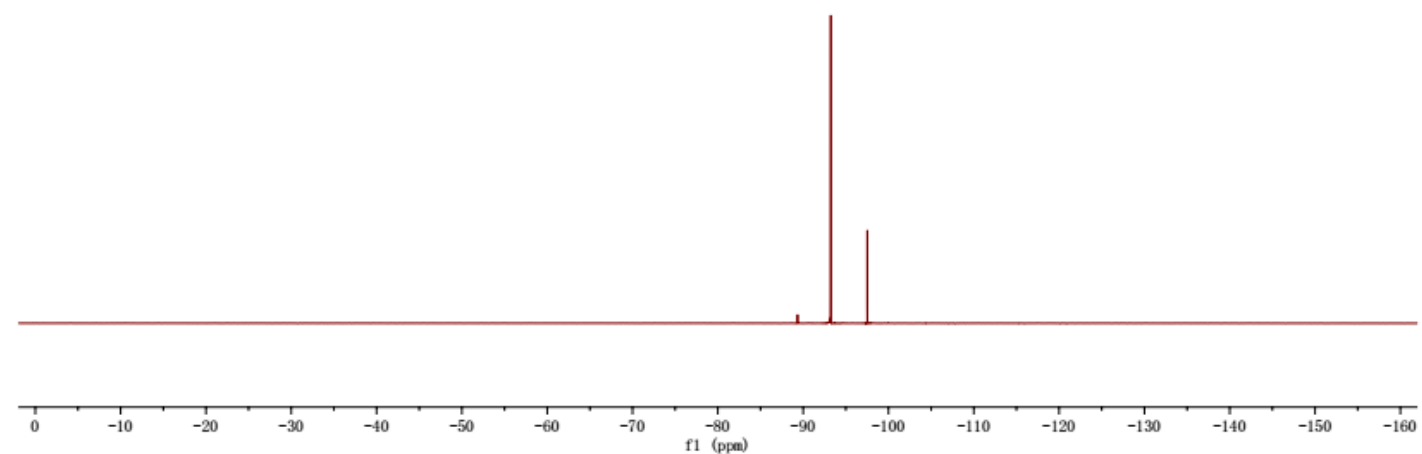

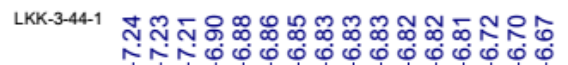

묘요

กุำฺำ<smiles>CCOC(=O)C(F)(F)C=C(I)c1cccc(OC)c1</smiles>

20

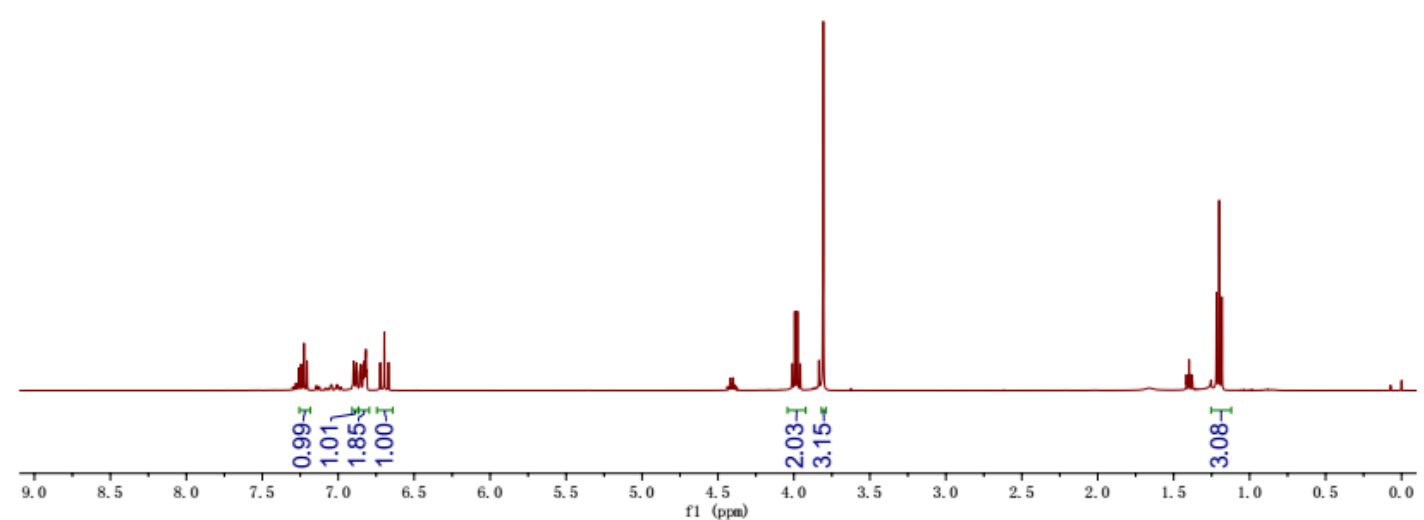



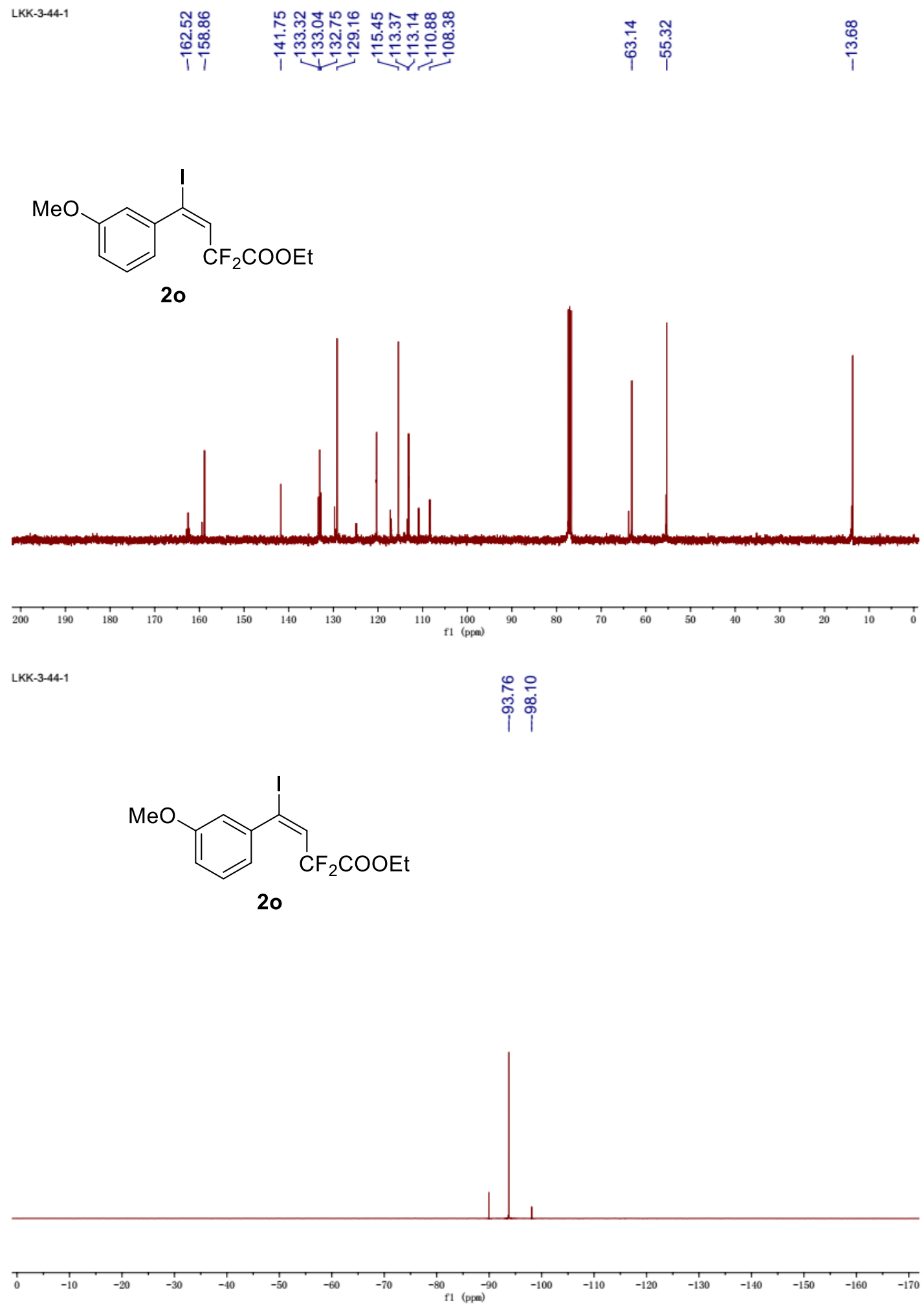

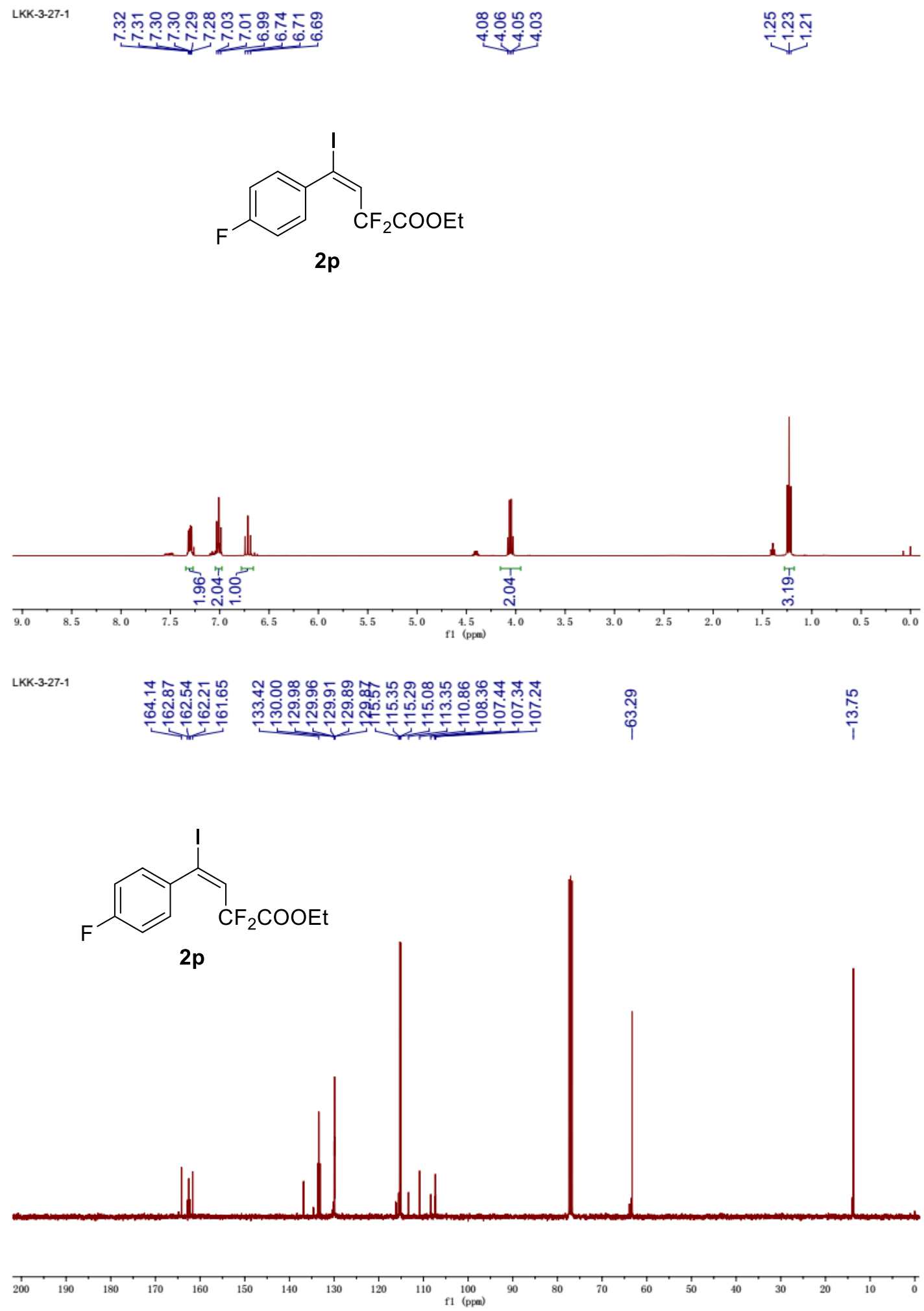
LKK-3-27-1

m<smiles>CCOC(=O)C(F)(F)C/C(I)=C(/I)c1ccc(F)cc1</smiles>
$2 p$
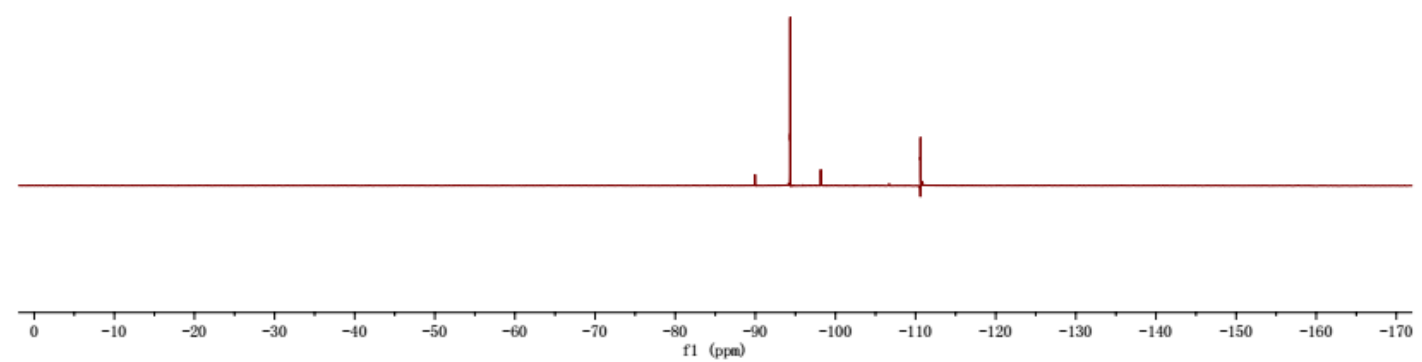

LKK-329-1

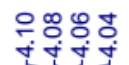

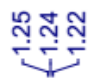<smiles>CCOC(=O)C(F)(F)C/C(I)=C(/I)c1ccc(Cl)cc1</smiles>

$2 q$

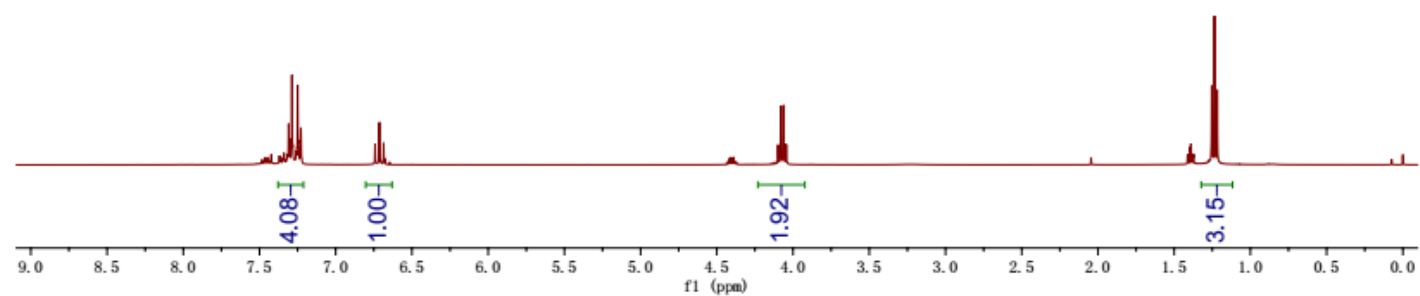



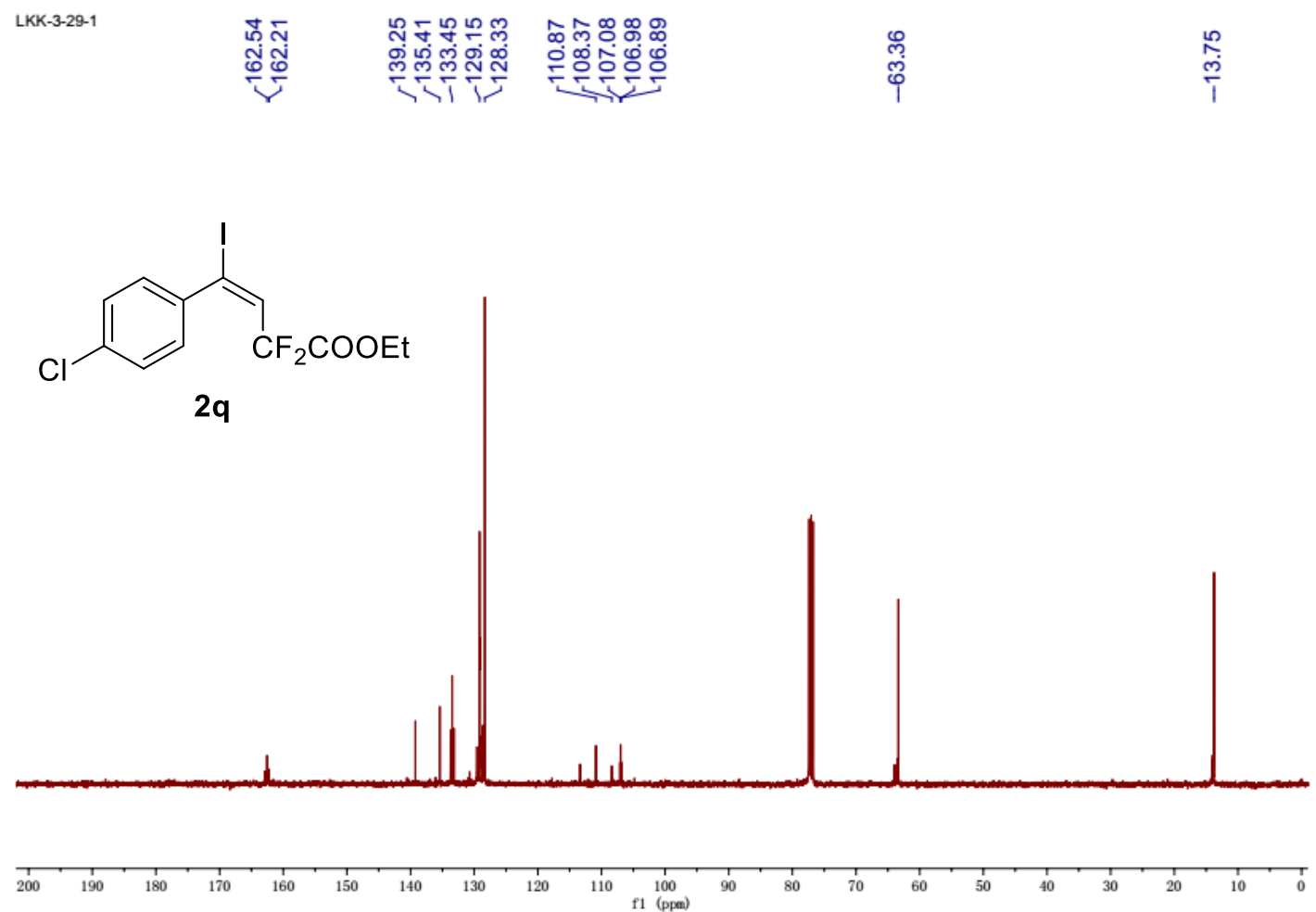

LKK-3-29-1

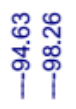
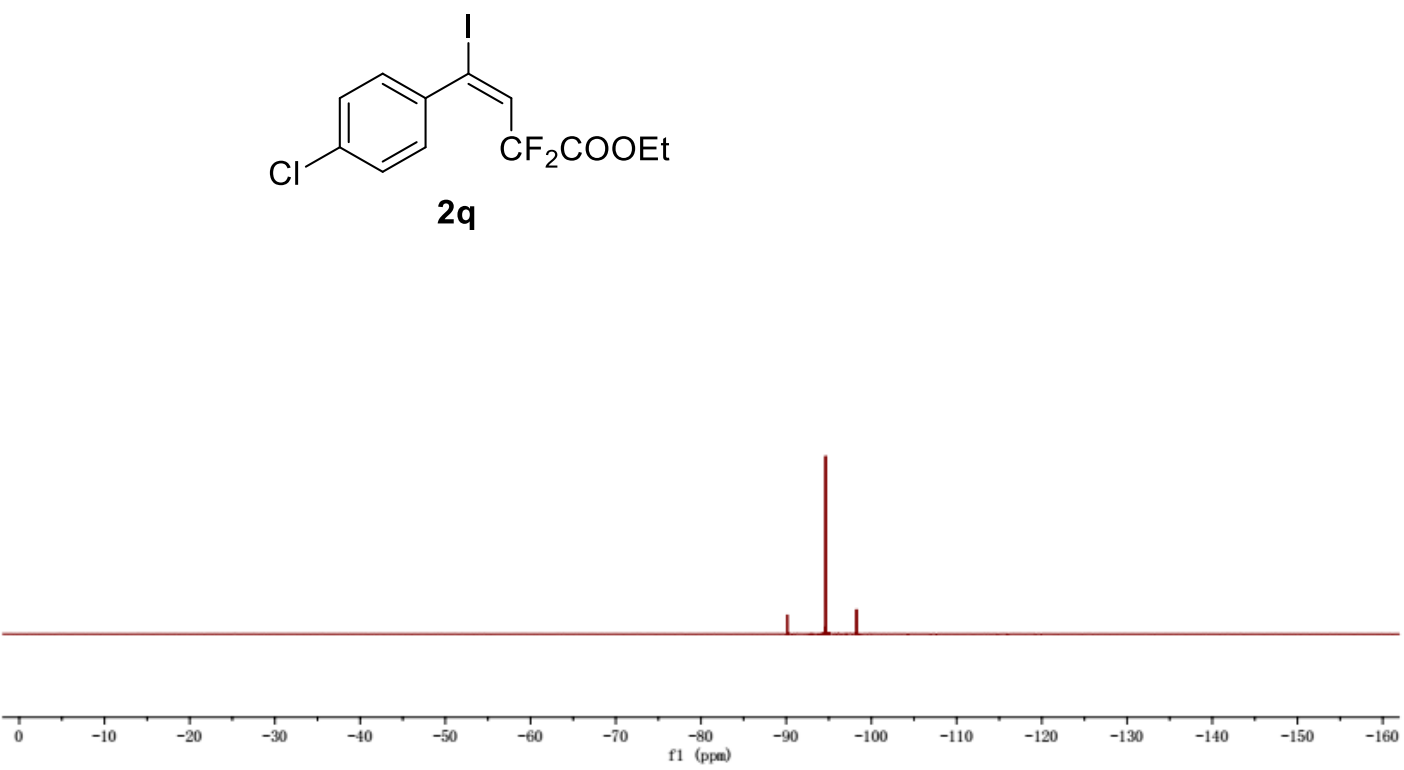
<smiles>CCOC(=O)C(F)(F)C=C(I)c1ccc(Br)cc1</smiles>

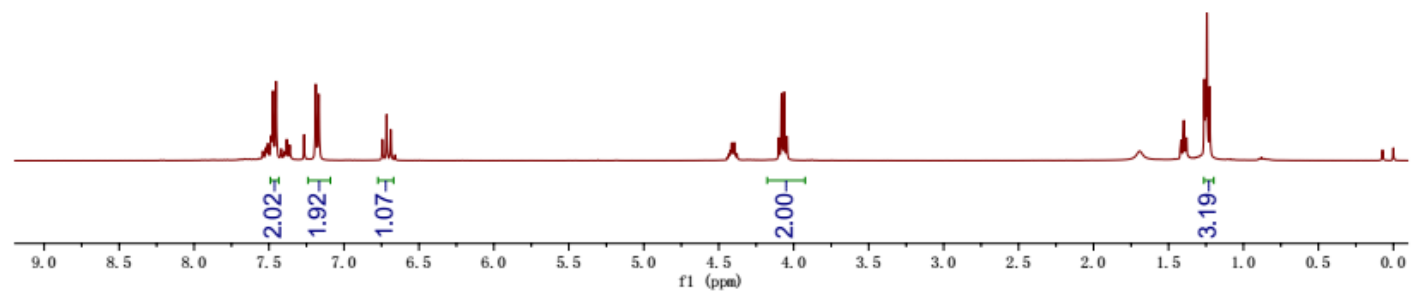

LKK-3-31-1A<smiles>CCOC(=O)C=C(C)c1ccc(Br)cc1</smiles>
$2 r$

$\frac{1}{200}$

19
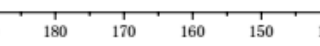

$140 \quad 1$

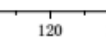

$110 \quad 100$ 
<smiles>CCOC(=O)C(F)(F)C=C(I)c1ccc(Br)cc1</smiles>

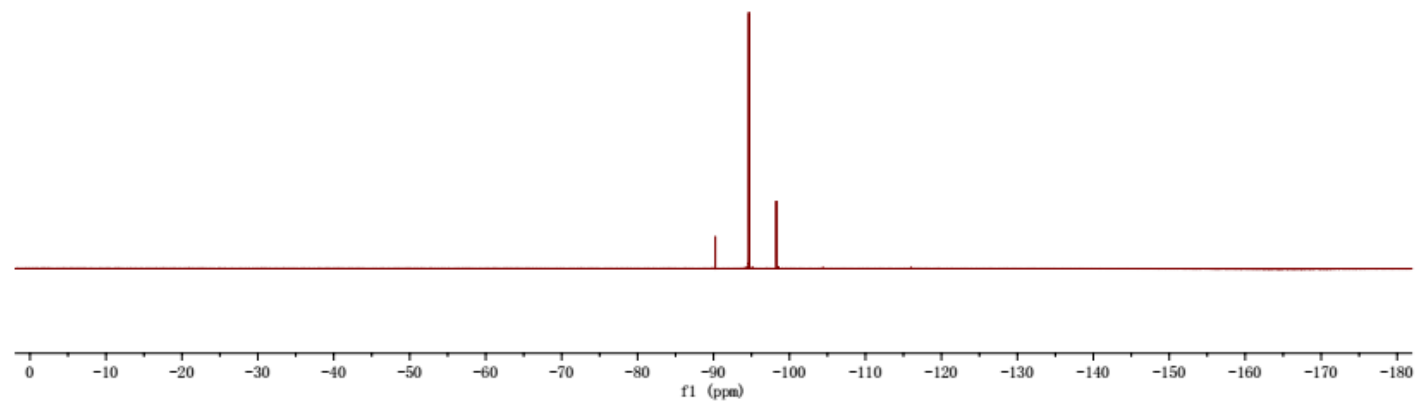

LKK-3-29-2

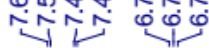

$\stackrel{+}{ }$

ํํำ<smiles>CCOC(=O)C(F)(F)/C=C(/I)c1ccc(C(F)(F)F)cc1</smiles>

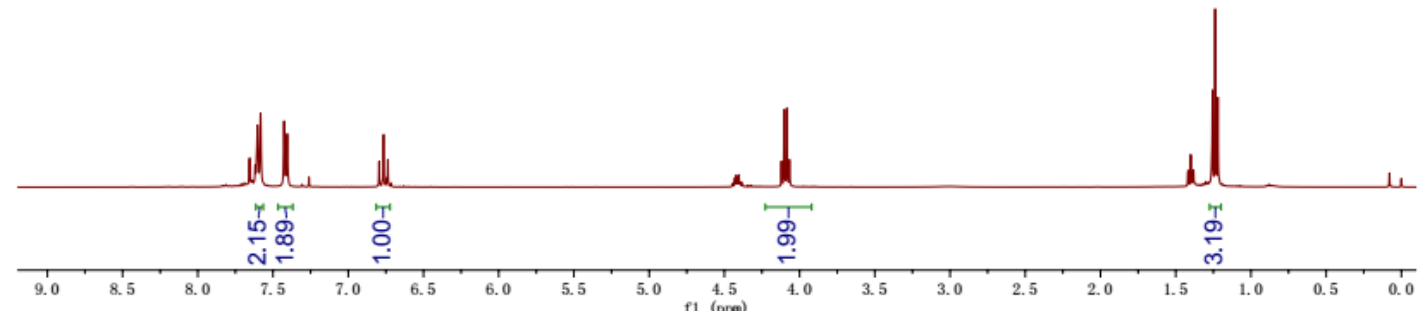


<smiles>CCOC(=O)[C-](C=C(I)c1ccc(C(F)(F)F)cc1)c1ccccc1</smiles>
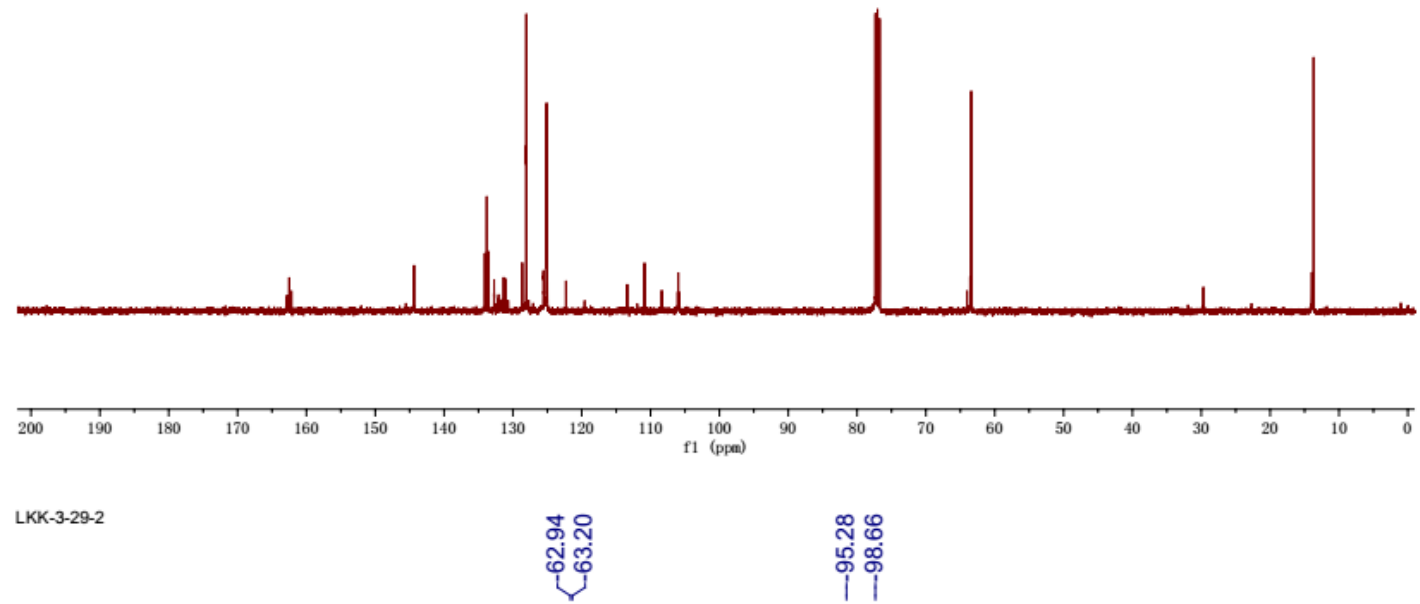<smiles>CCOC(=O)[C-](C=C(I)c1ccc(C(F)(F)F)cc1)CC</smiles>

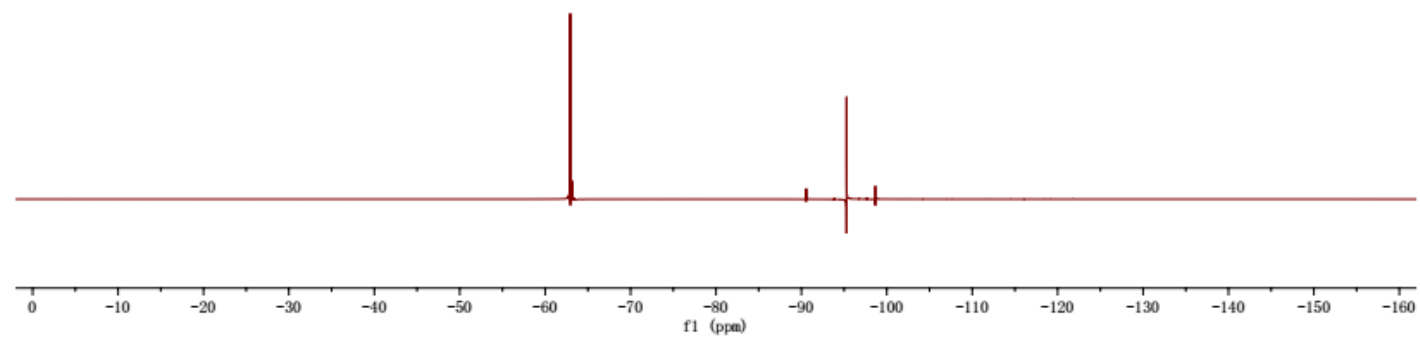



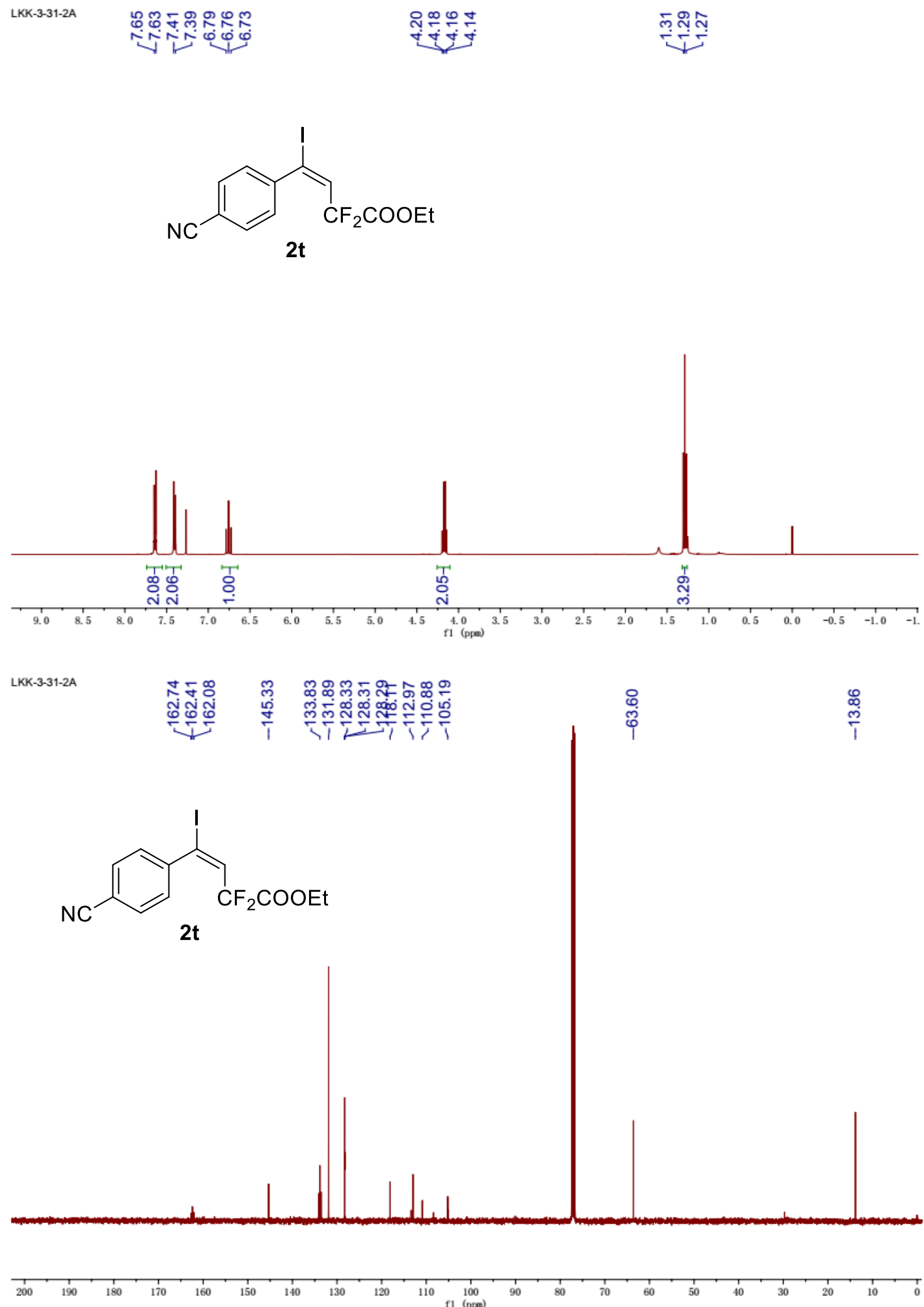

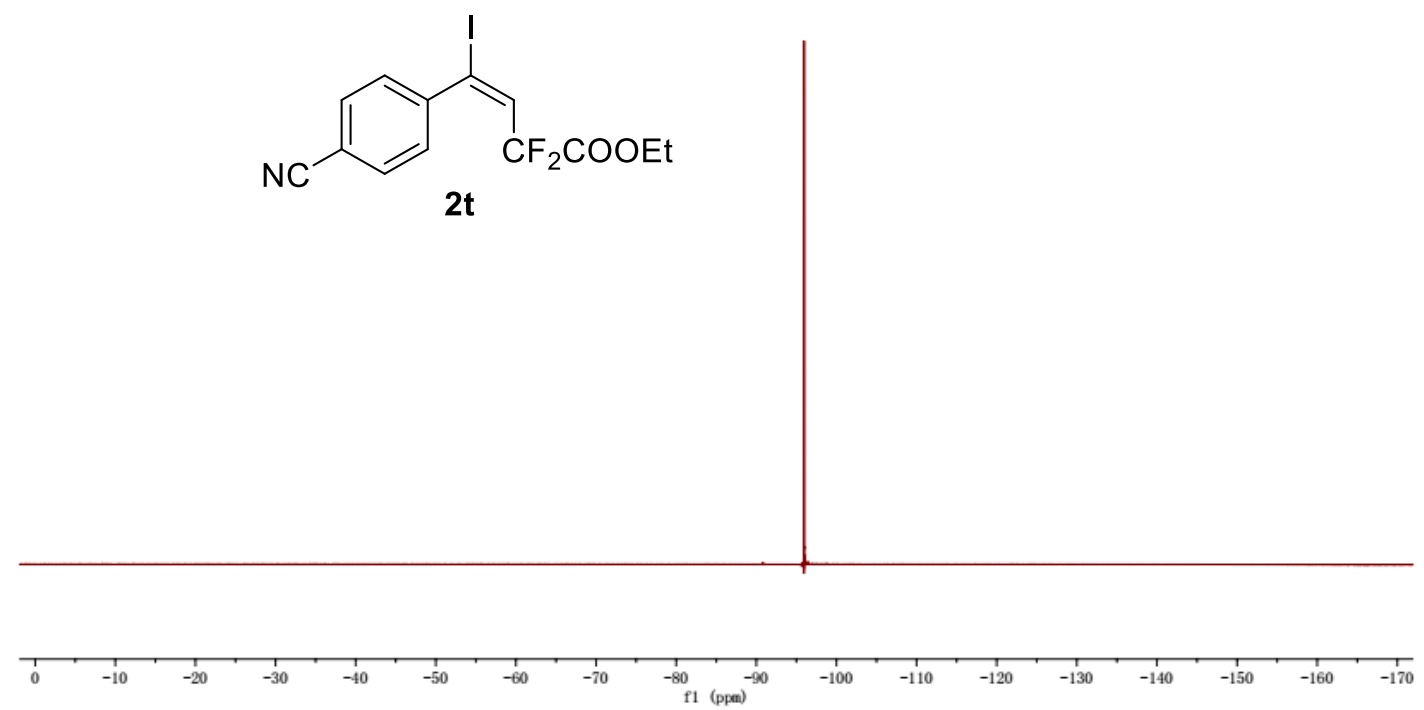

LKK-3.3.

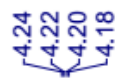

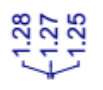
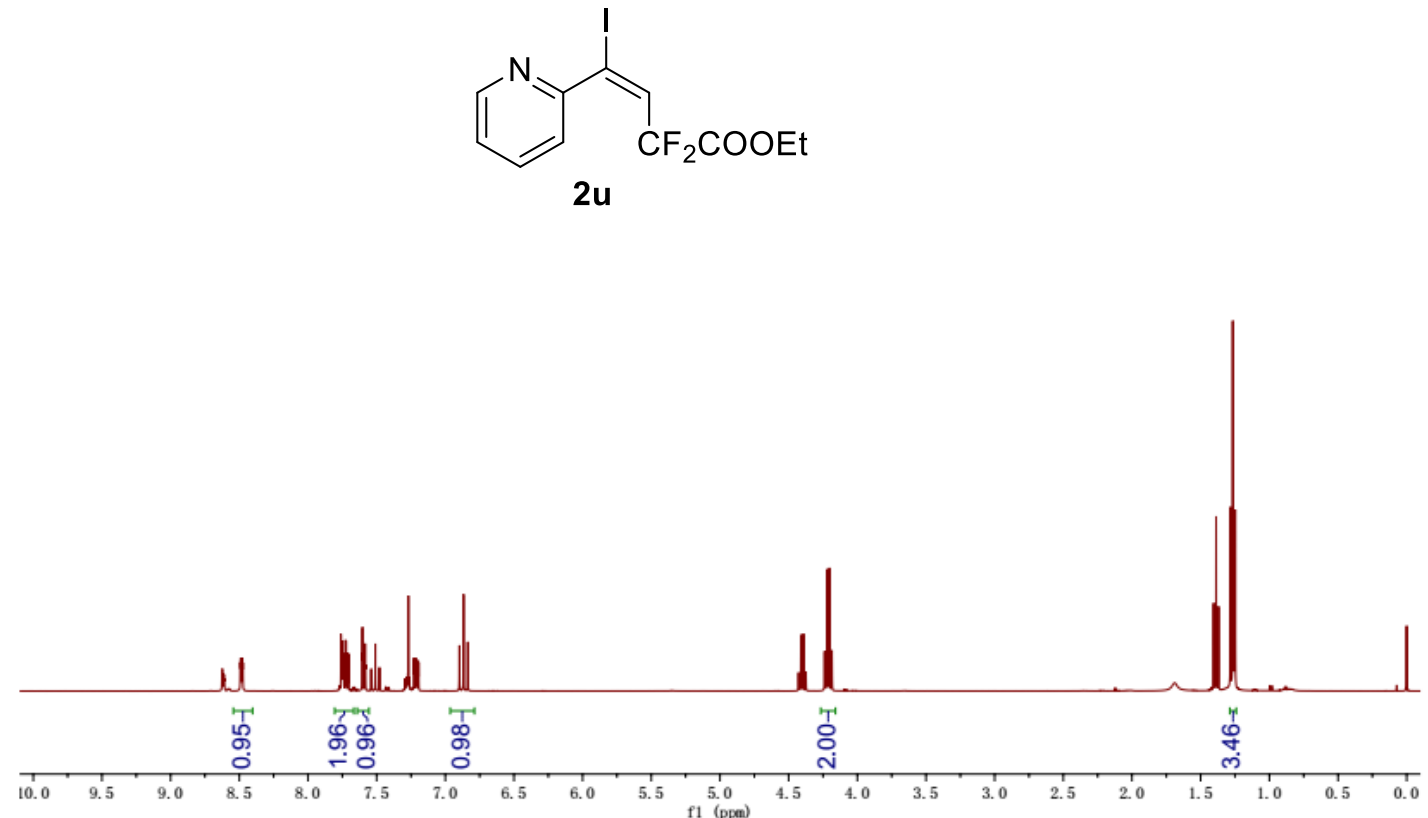
LKK-3-33-1

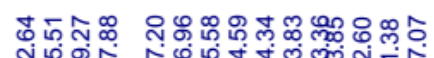

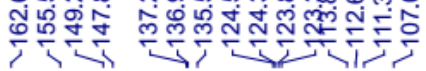

กัฒ

ฏ્ఝ

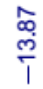<smiles>CCOC(=O)C(F)(F)C=C(I)c1ccccn1</smiles>

$2 \mathrm{u}$

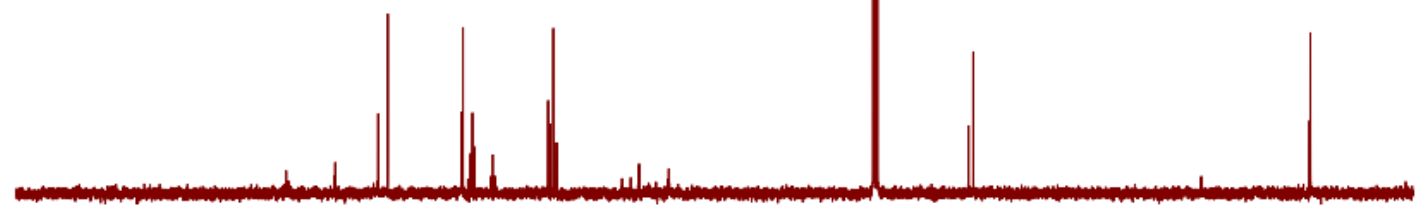

\section{$\frac{1}{200}$}

LKK-3-33-1
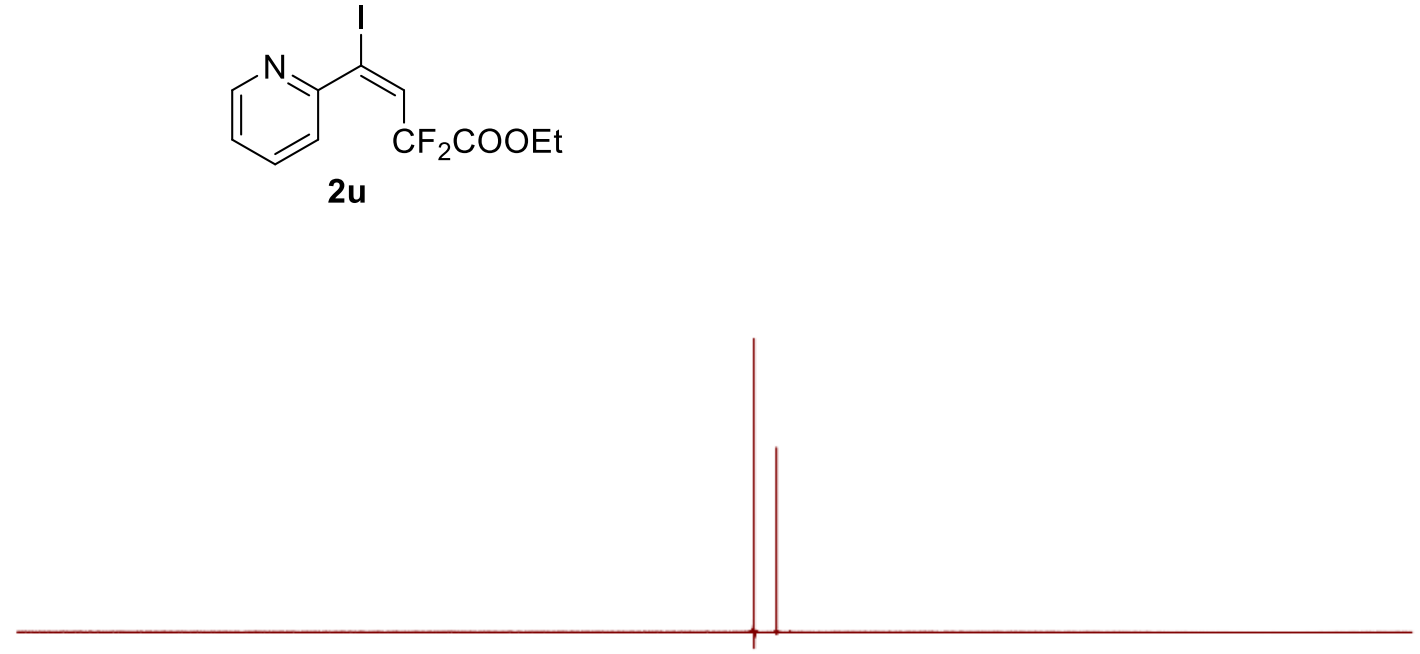

T

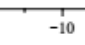

$\frac{1}{-10} \cdot \frac{1}{-30}$

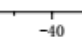

$-50$ f1 $\stackrel{-90}{\text { (ppan) }}$ 
LKK-3-37-1
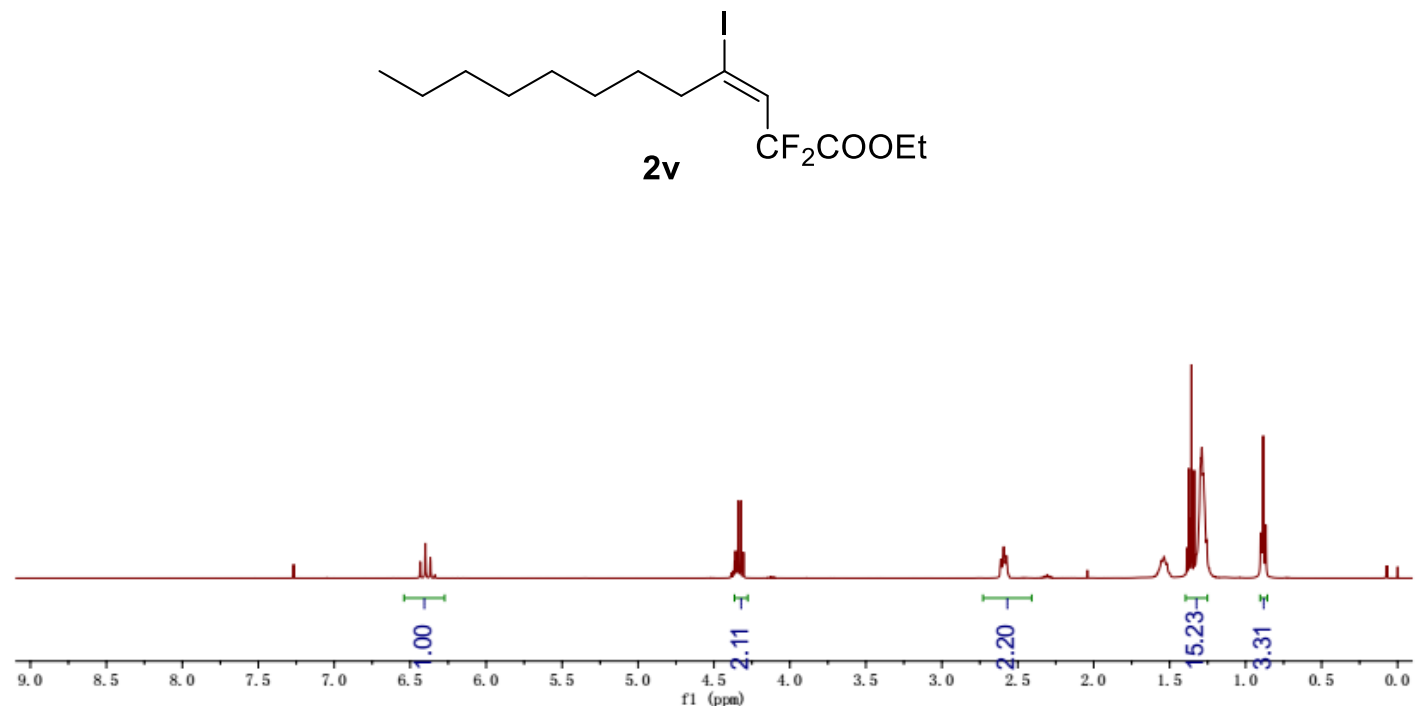

LKK-3-37-1
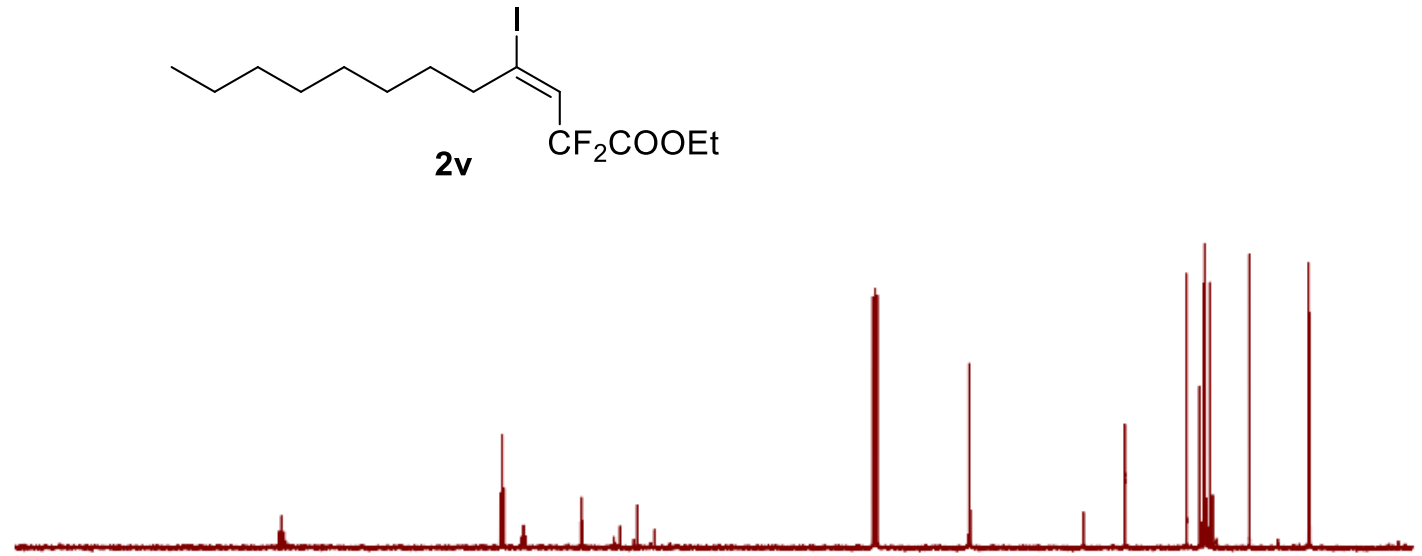

$\frac{1}{200}$
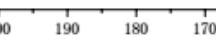

${ }_{160}^{1} \cdot 150$

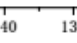

${ }_{120}^{1}$ f1 $\stackrel{100}{(\mathrm{ppa})}$ 

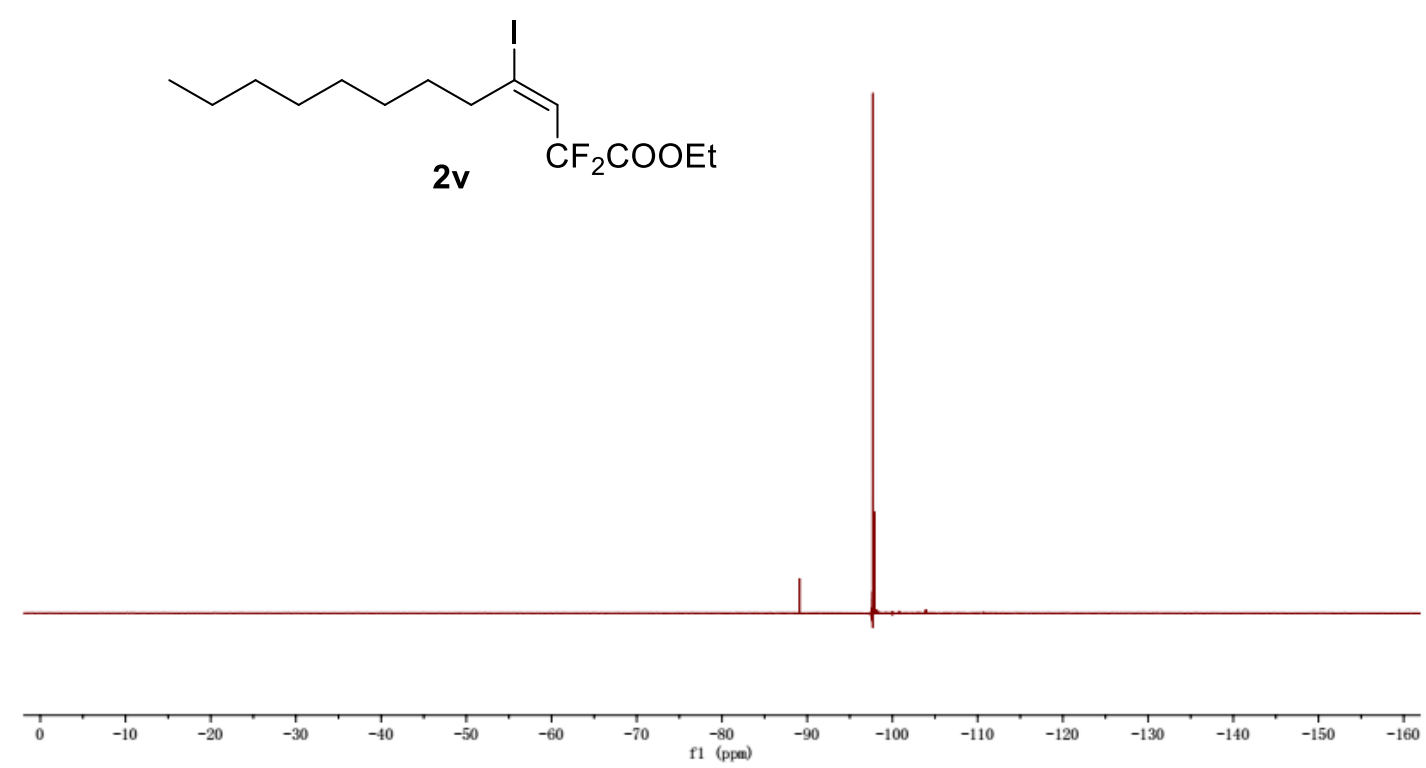

LKк-3.<smiles>CCOC(=O)[C+](C)/C(C)=C/c1ccccc1</smiles>

$3 a$

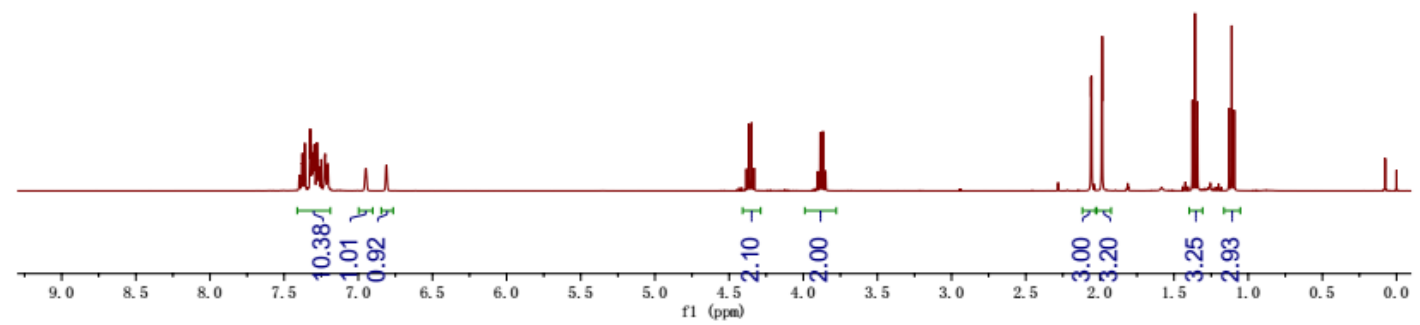



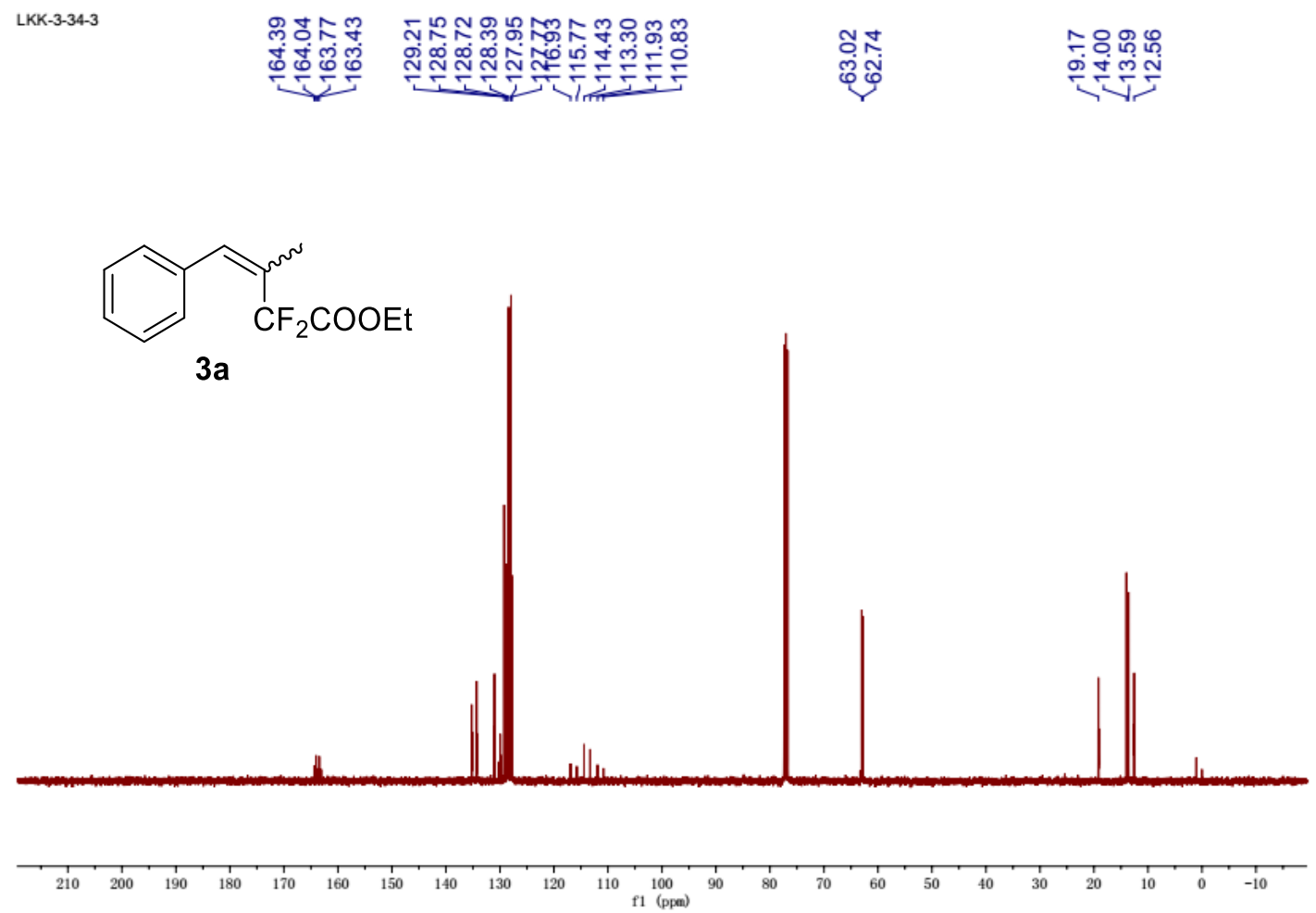

LKK-3-34-3

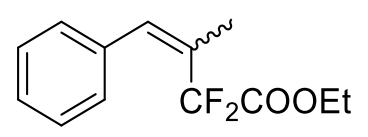

$3 a$
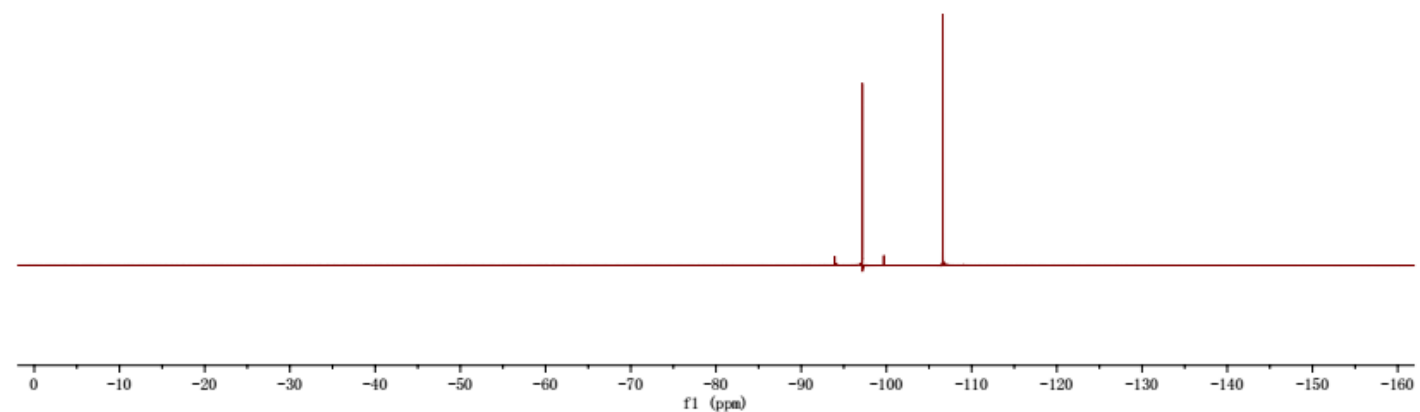
LKK-3-63-1

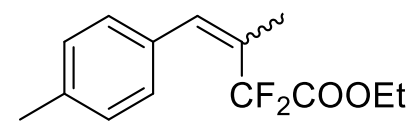

$3 b$

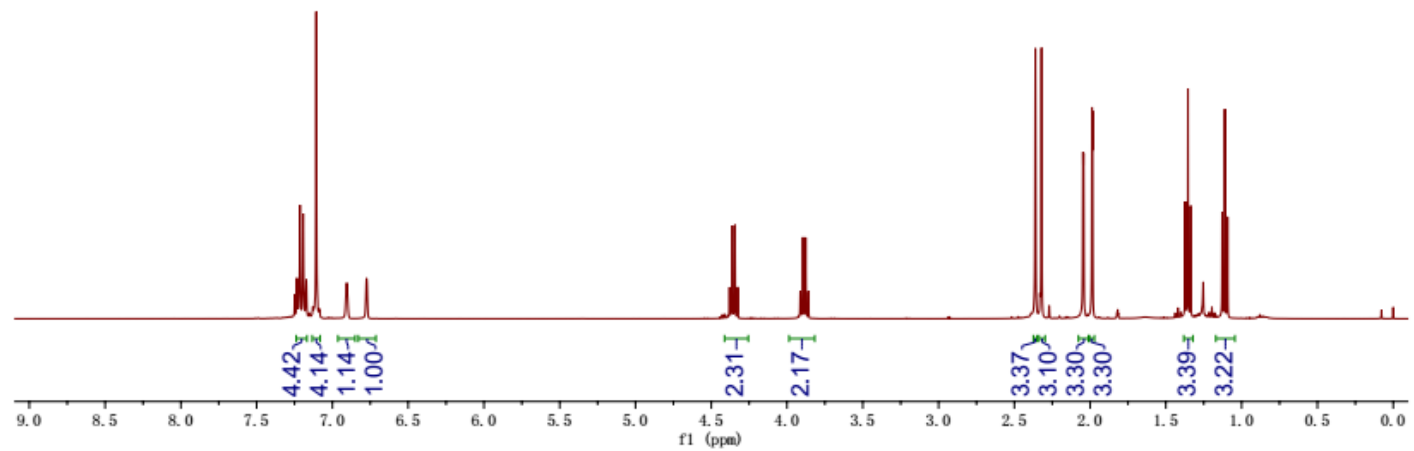<smiles>CCOC(=O)C(C)(C)/C(C)=C/c1ccc(C)cc1</smiles>

$3 b$

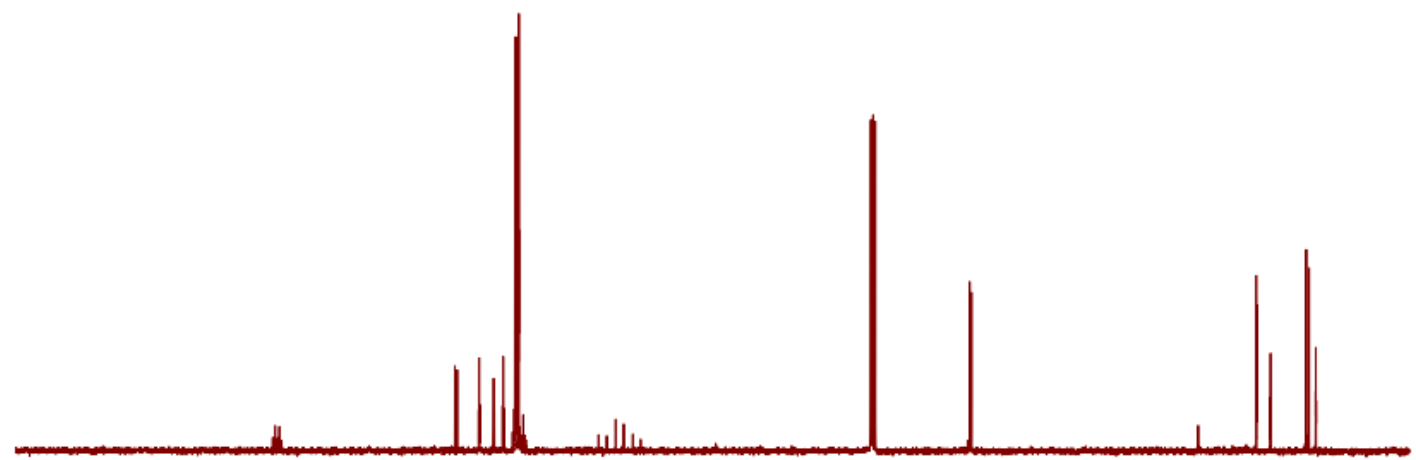

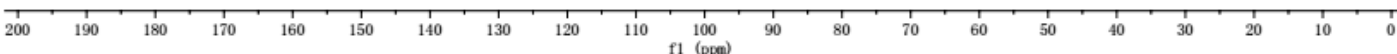


LKK-3-63-1<smiles>CCOC(=O)C(F)(F)C(C)=Cc1ccc(C)cc1</smiles>

3b

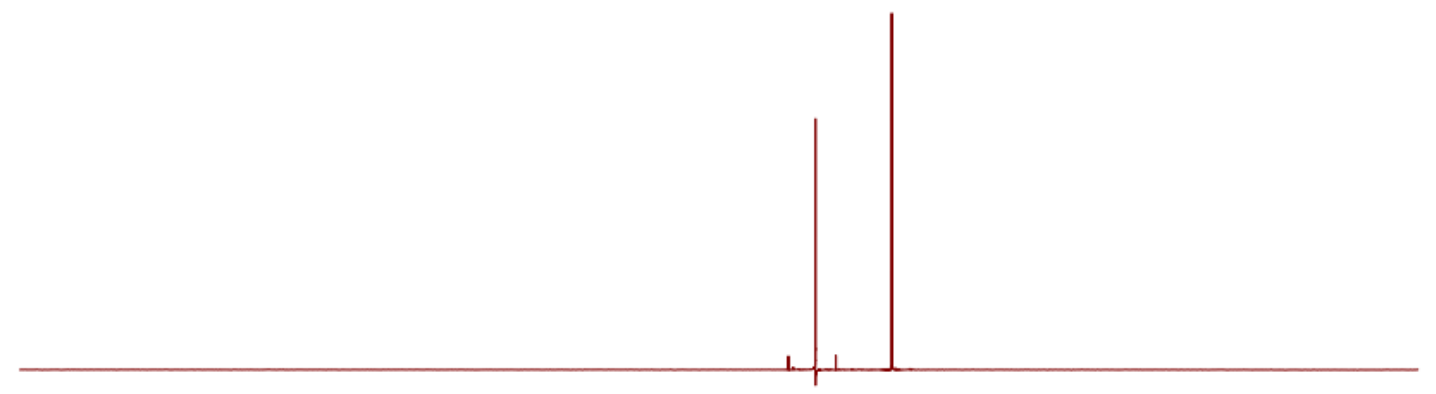<smiles>CCOC(=O)C(C)(F)C(C)=Cc1ccc(OC)cc1</smiles>

$3 c$

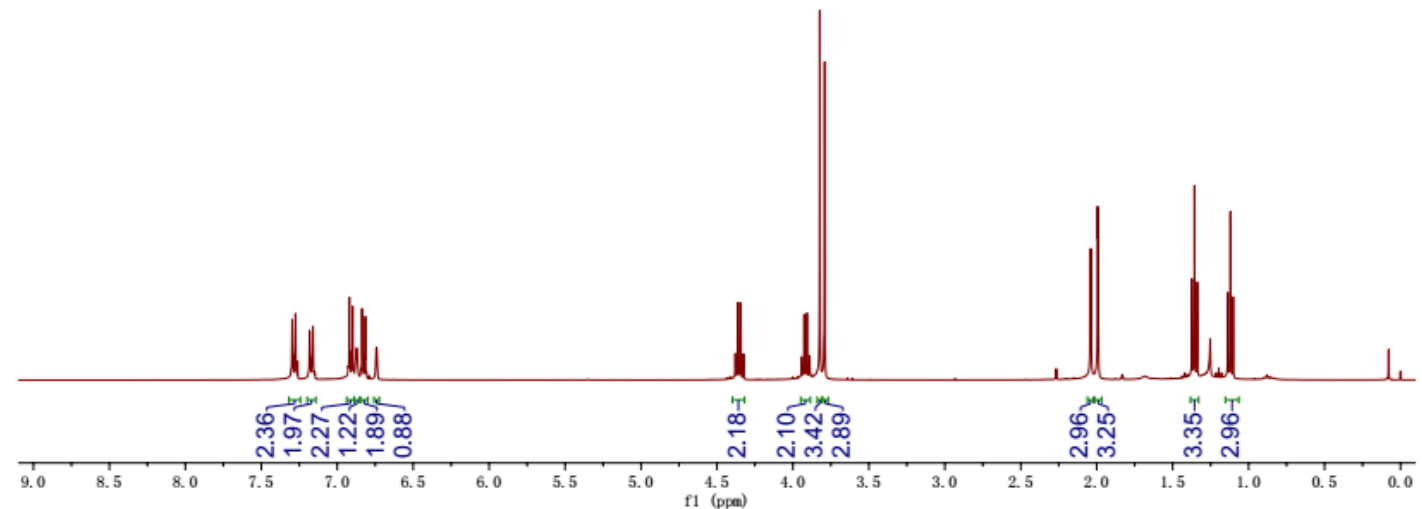



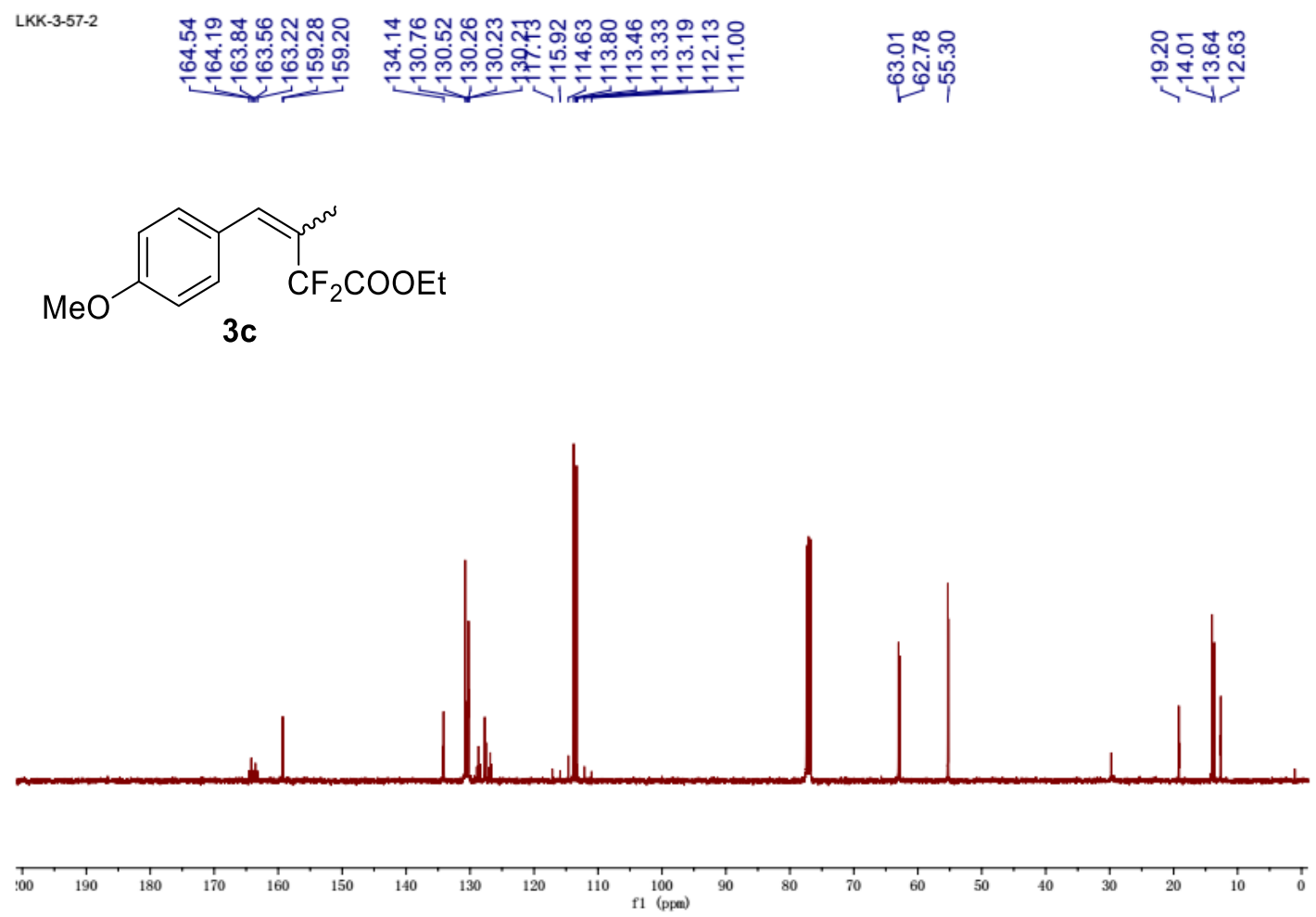

LKK-3-57-2<smiles>C=C(C=Cc1ccc(OC)cc1)C(=O)OCC</smiles>

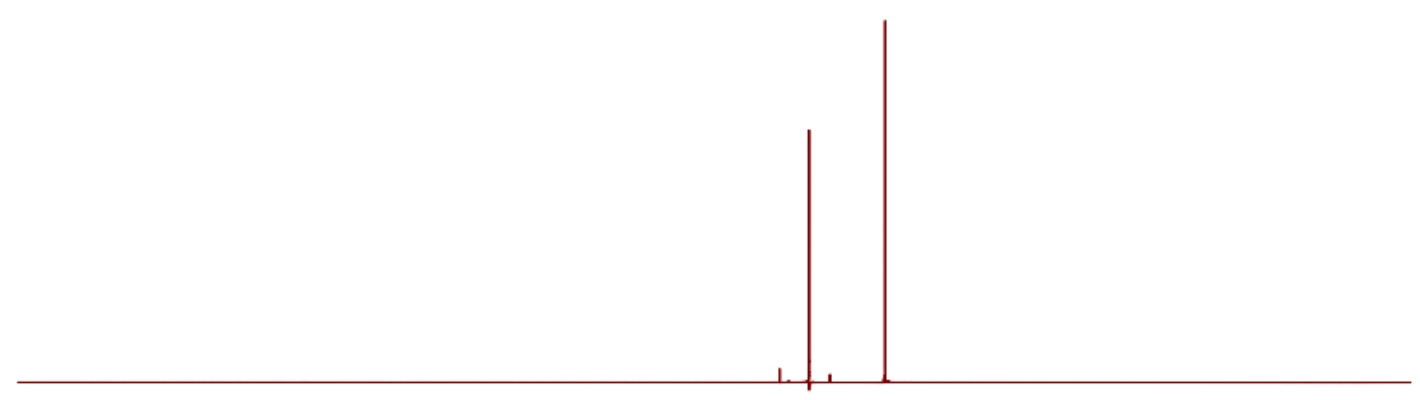

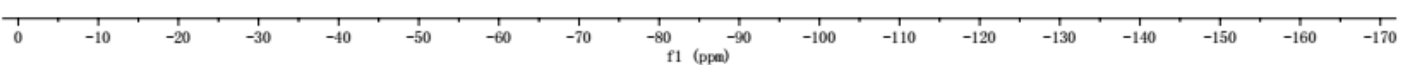




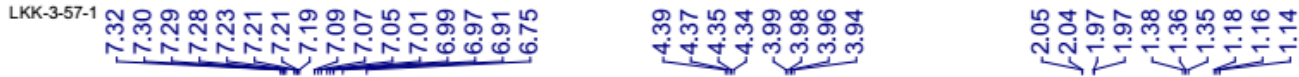
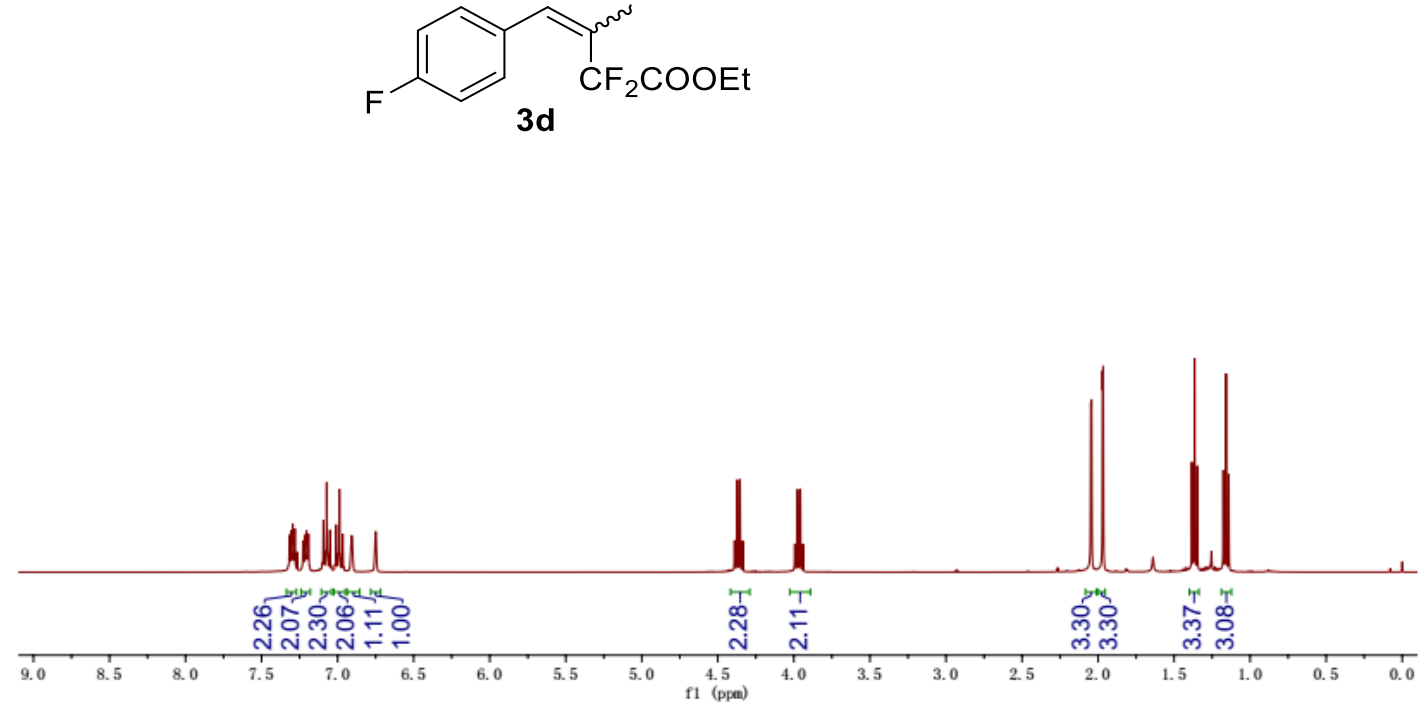

LKK-3-57-1

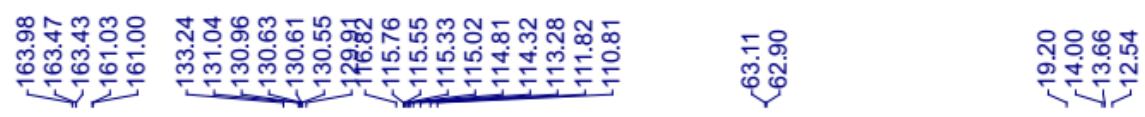

$\overbrace{3 \mathbf{C}}^{\mathrm{CF}_{2} \mathrm{COOEt}}$

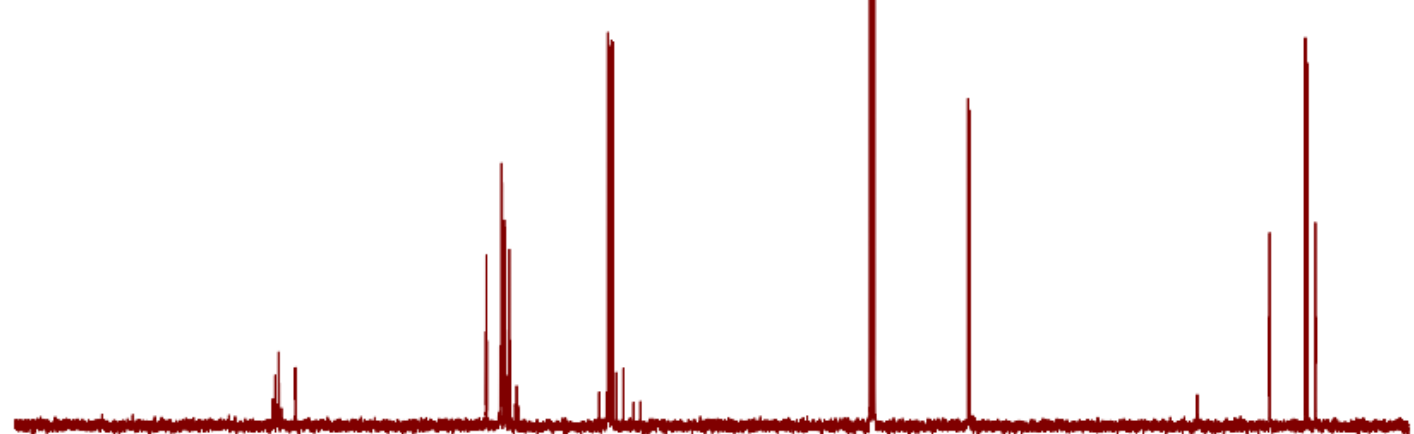

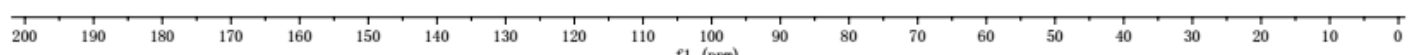



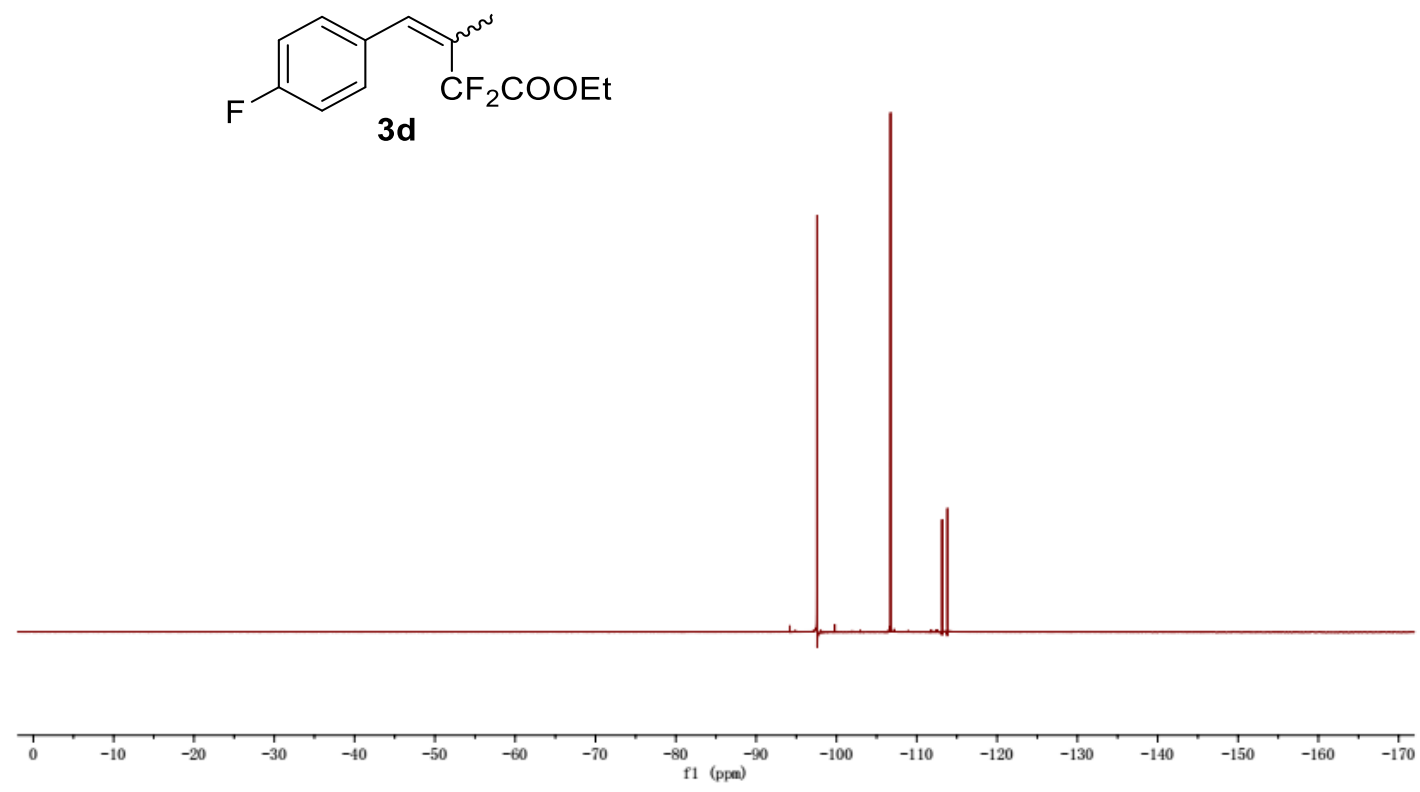

LKK-3-63-2

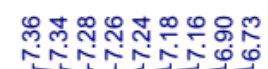

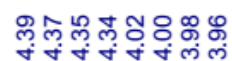

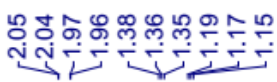<smiles>CCOC(=O)C(C)(F)C(C)=Cc1ccc(Cl)cc1</smiles>

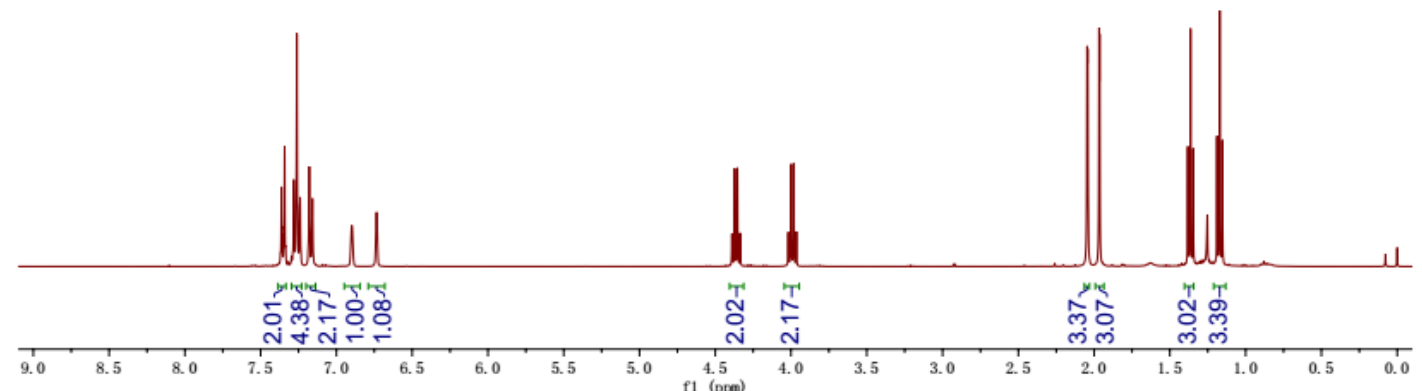



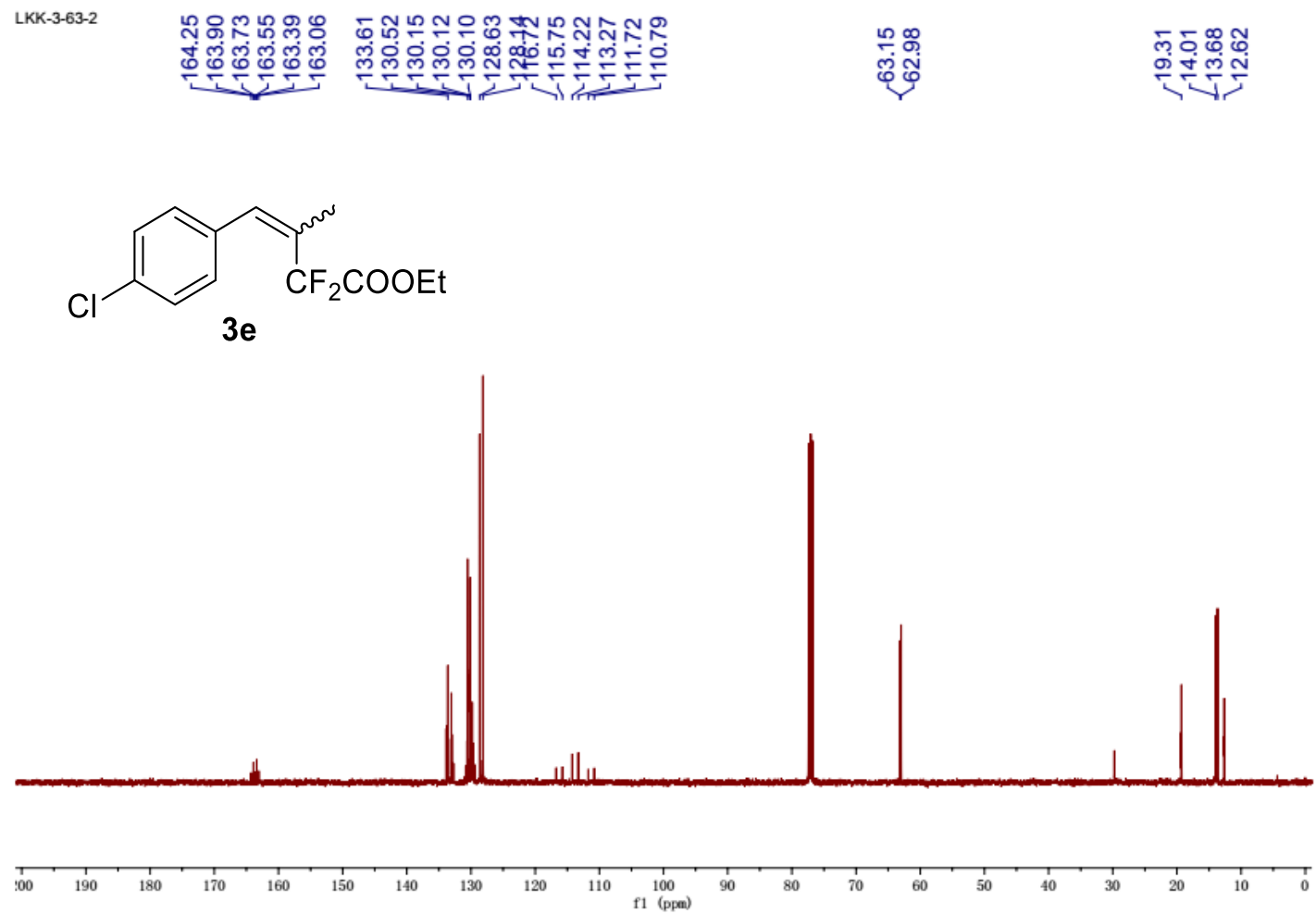

LKK-3-63-2
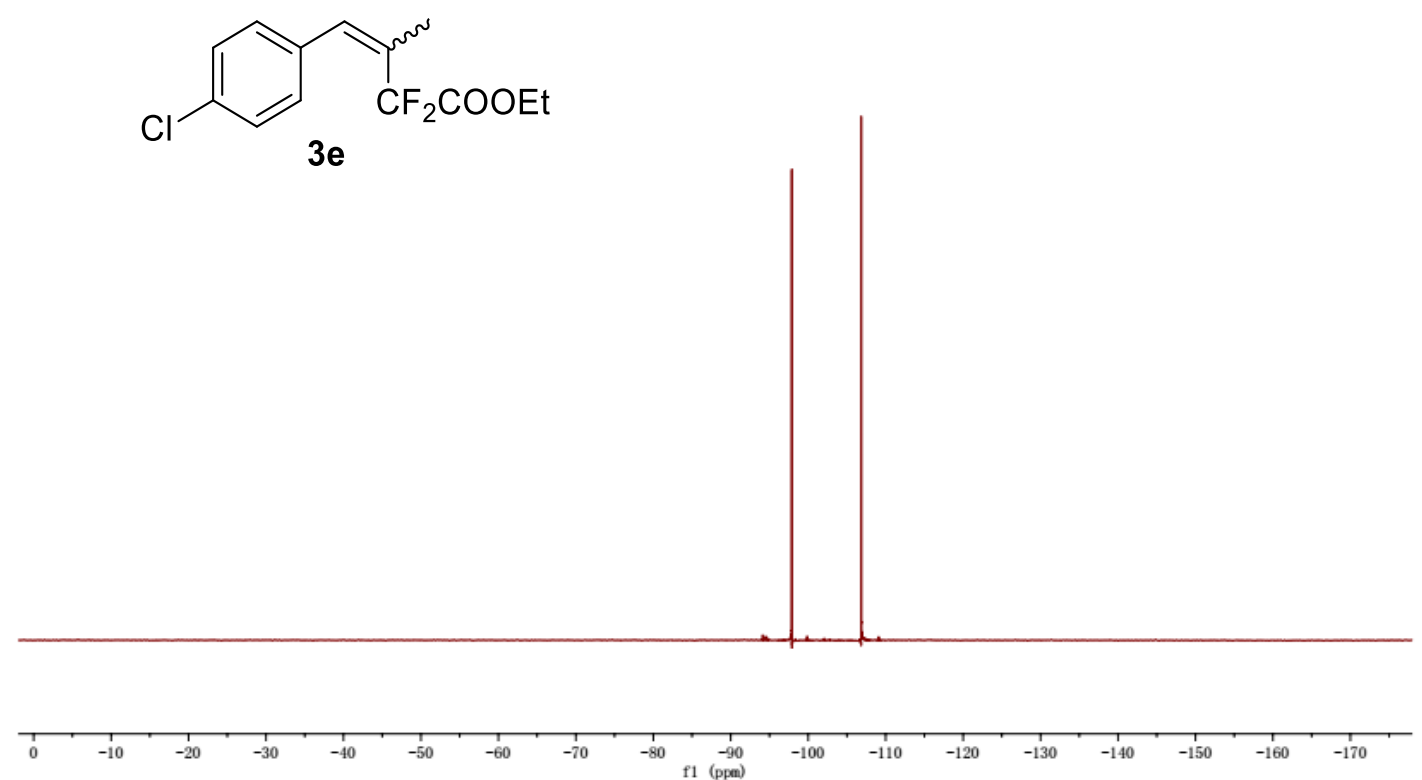

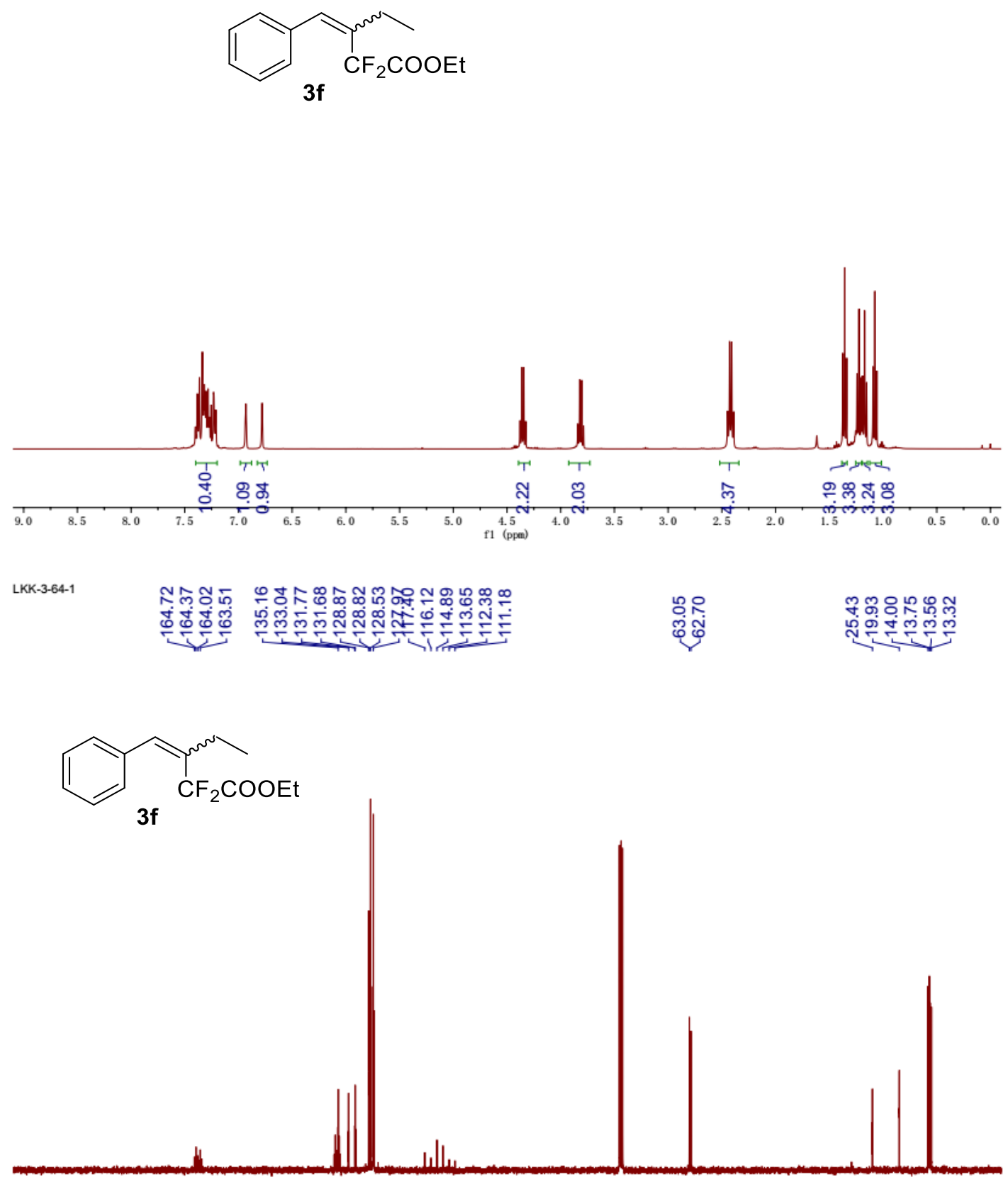

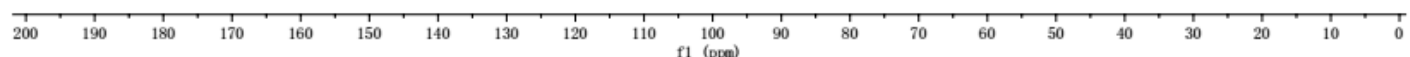


LKK-3-64-1

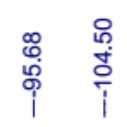

$\overbrace{3 f}^{m}$
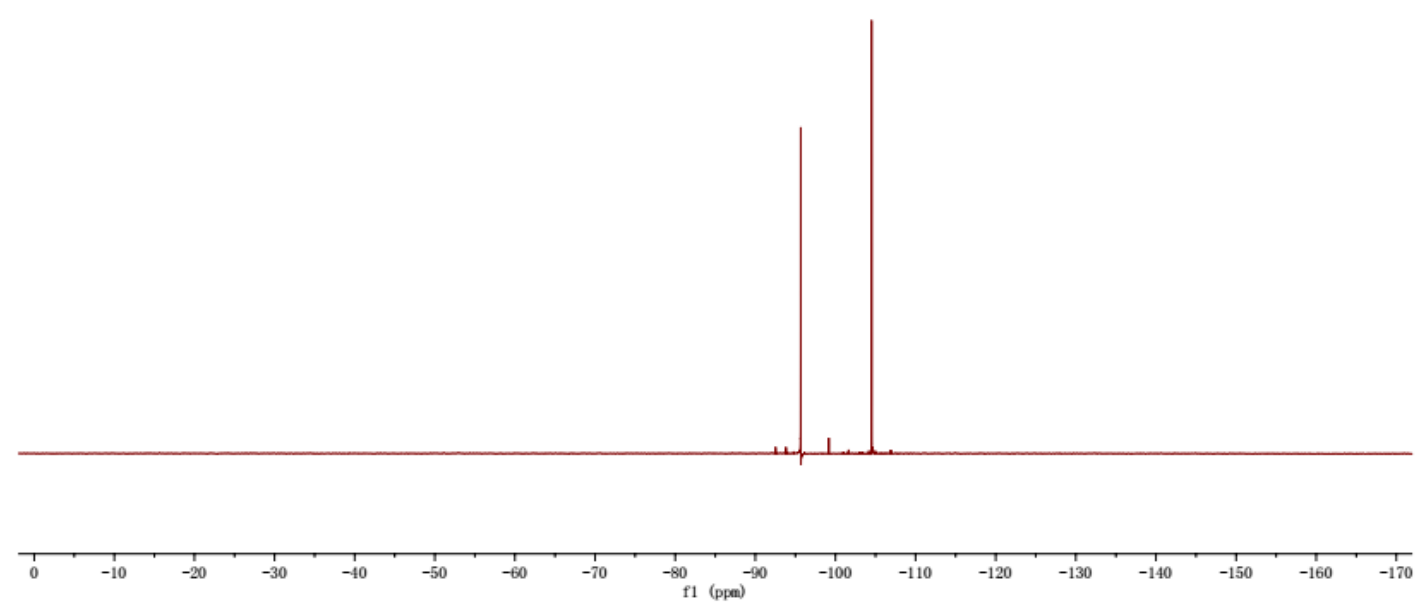

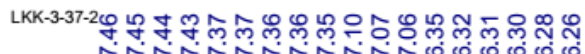

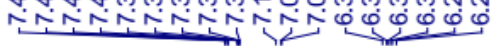

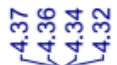

쌈

$\overbrace{\mathbf{5 a}}^{\mathrm{CF}_{2} \mathrm{COOEt}}$

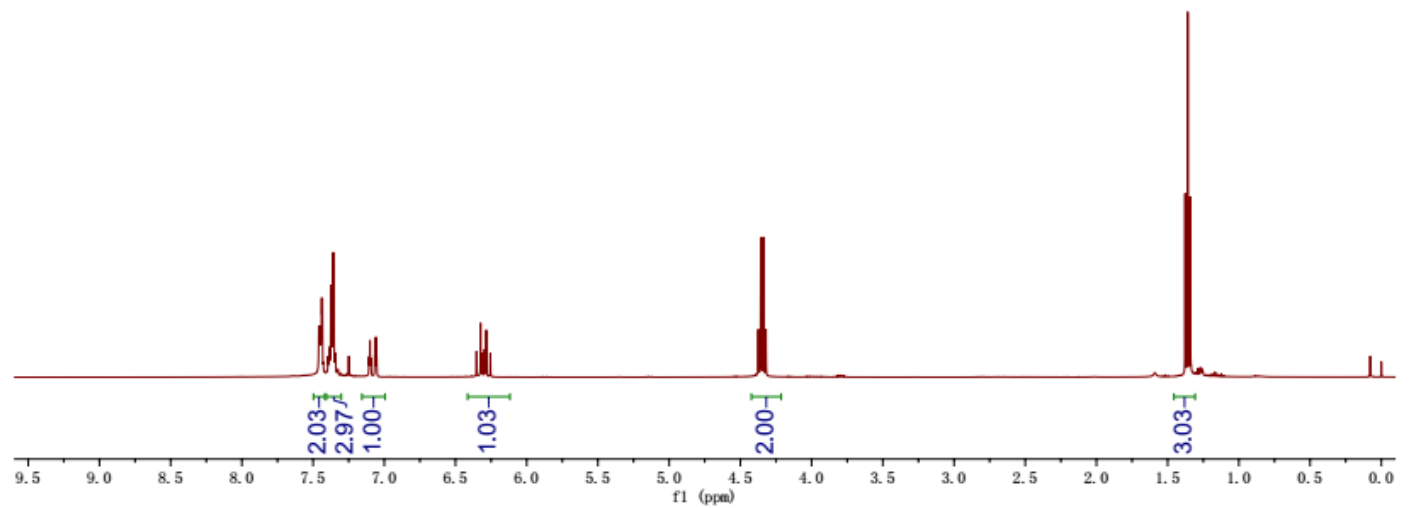



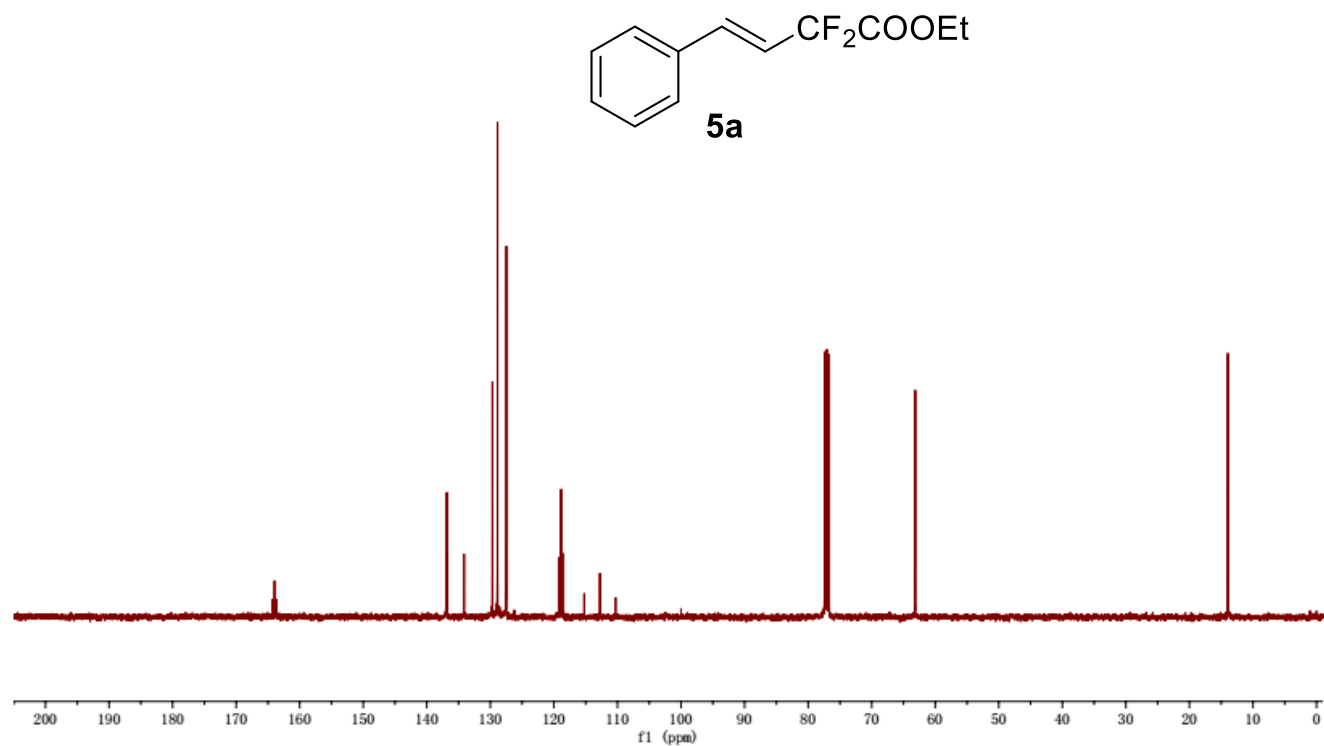

LKK-3-37-2

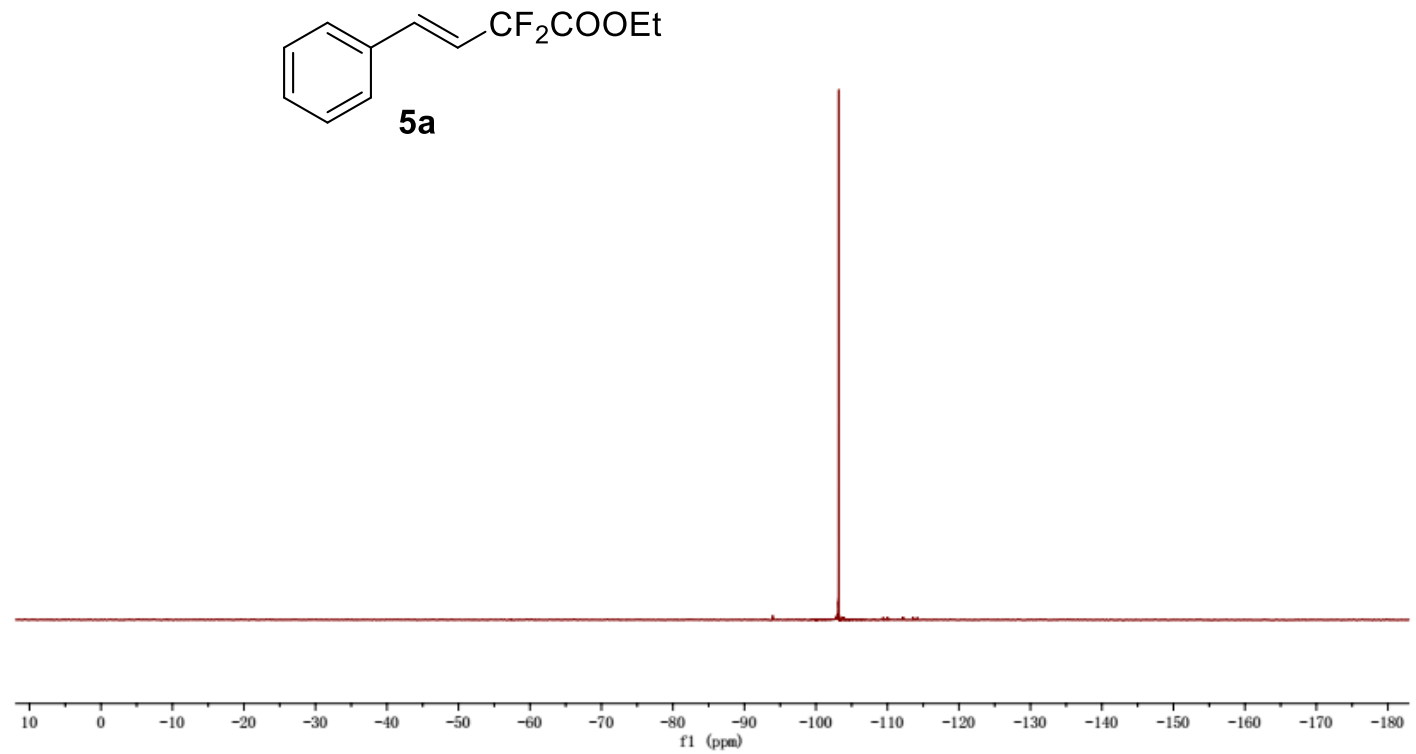



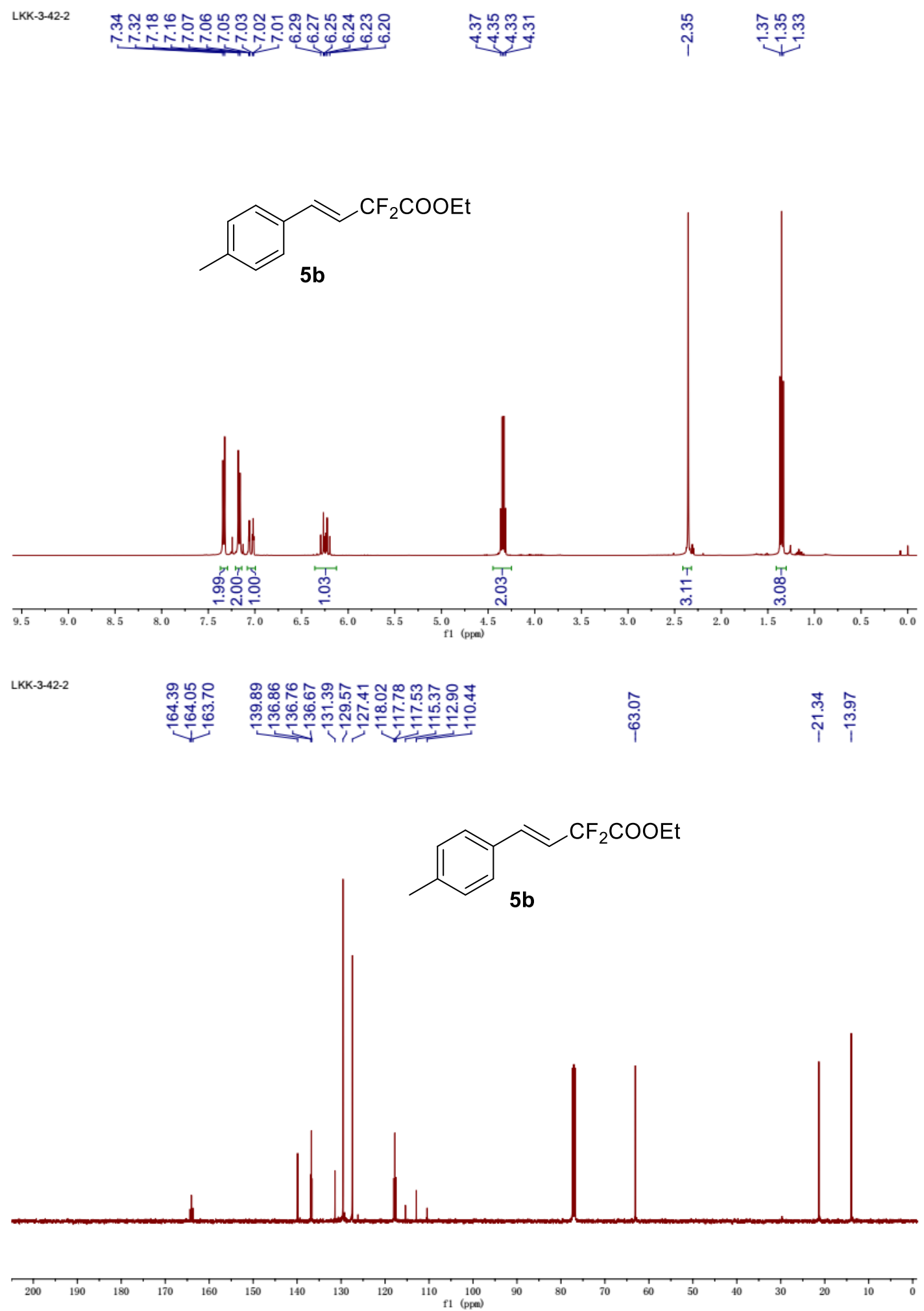


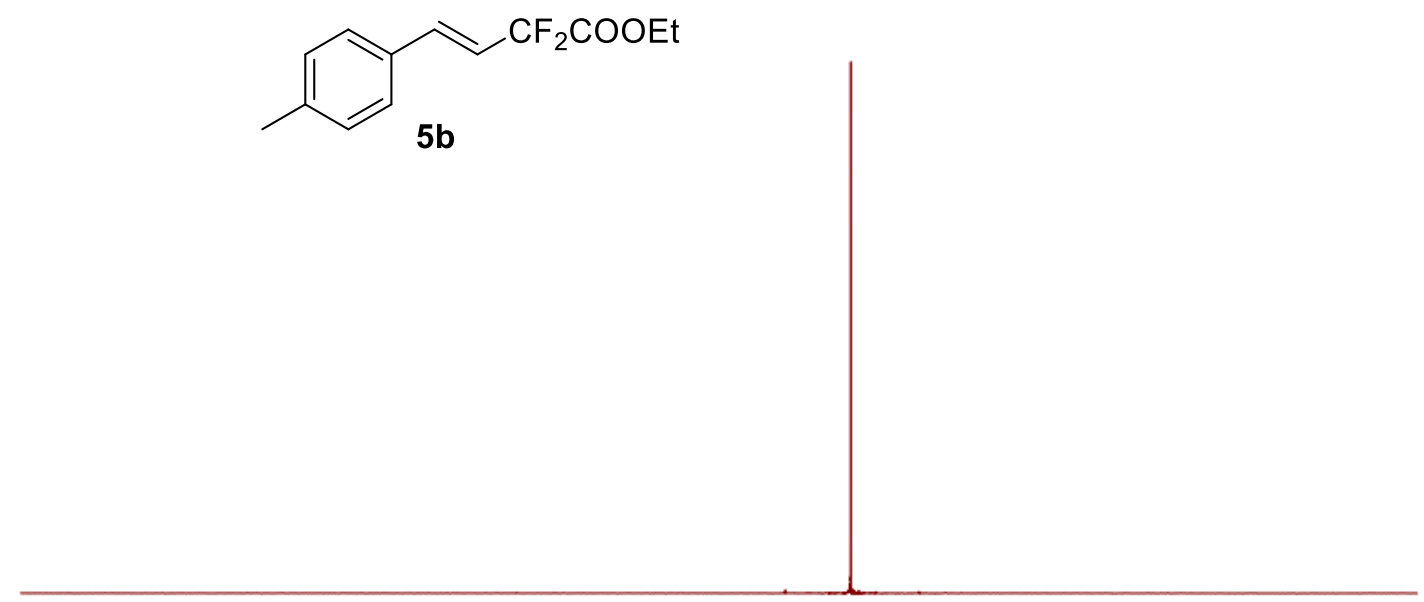$$
0
$$
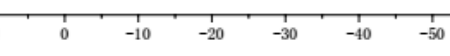

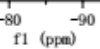

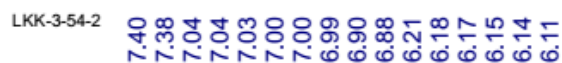

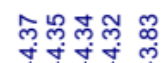

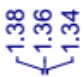<smiles>CCOC(=O)C(F)(F)C=Cc1ccc(OC)cc1</smiles>

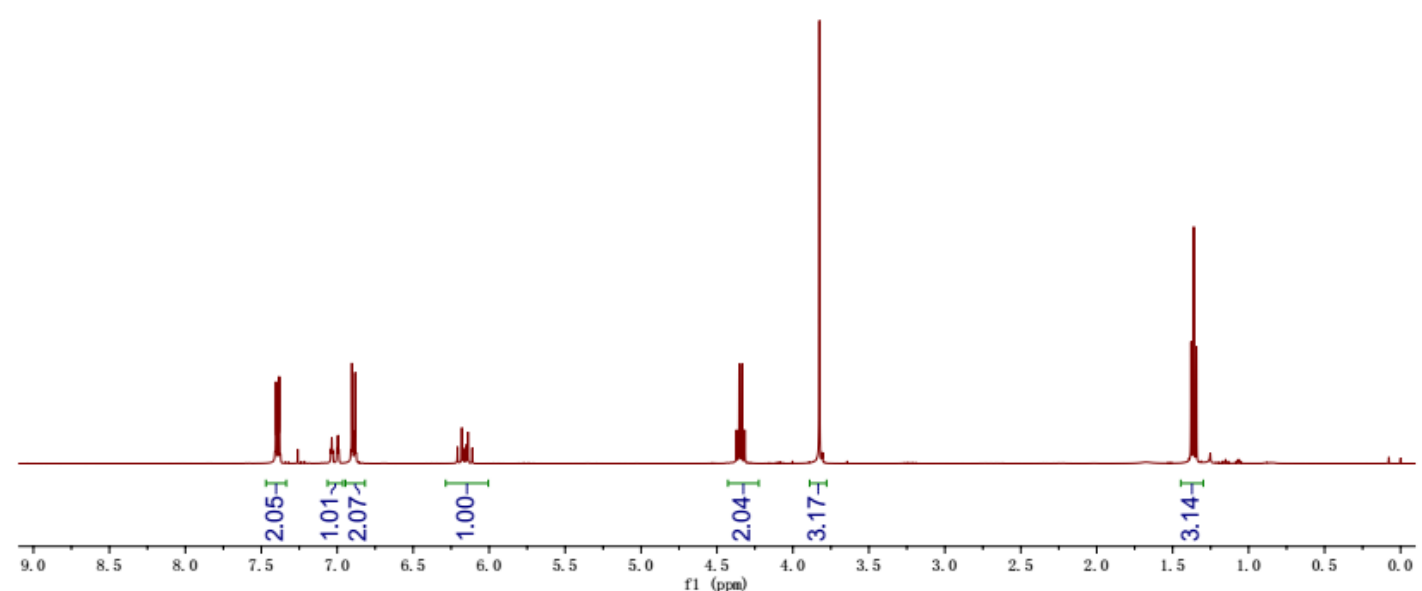



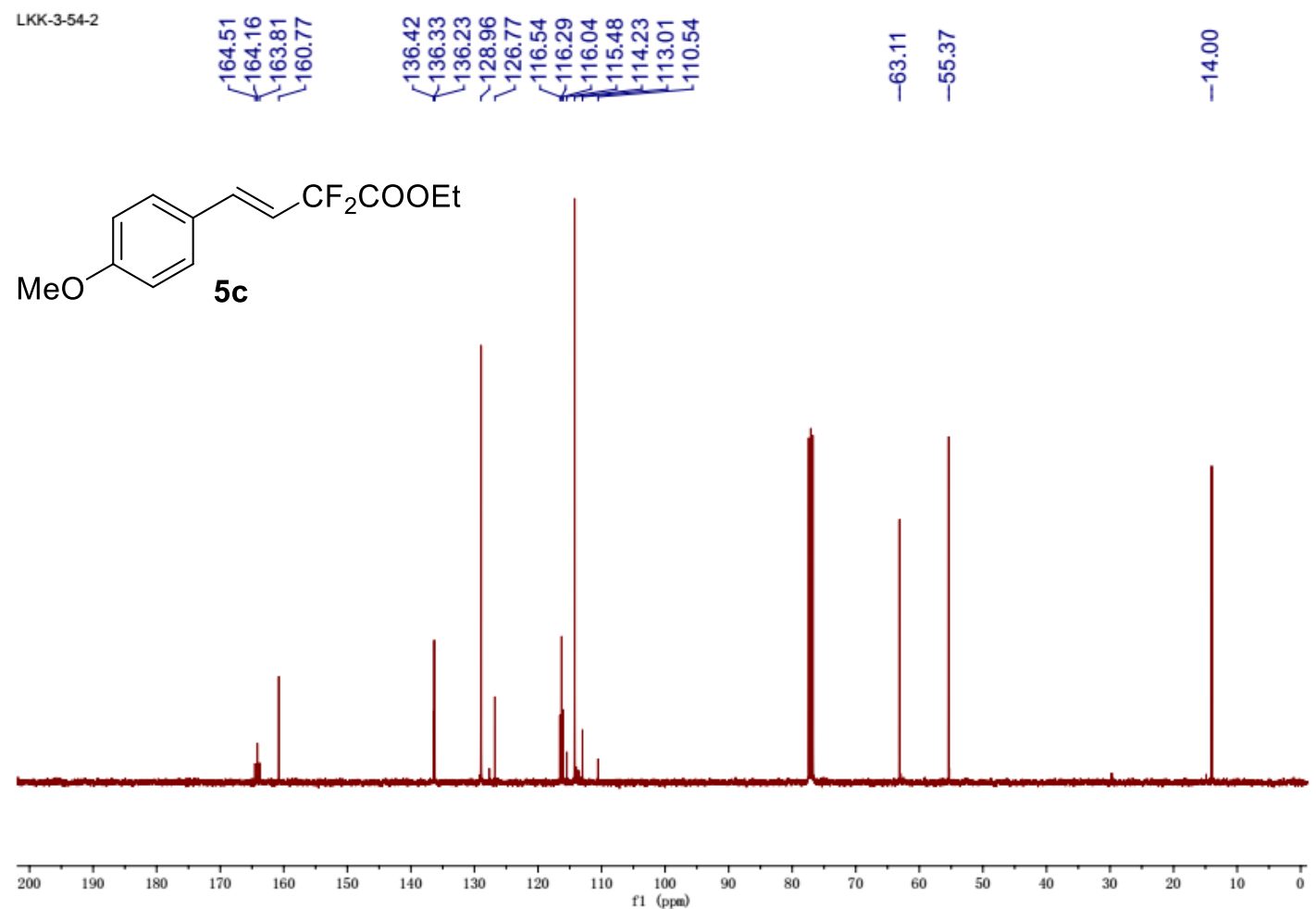

LKK-3-54-2<smiles>CCOC(=O)C(F)(F)C=Cc1ccc(OC)cc1</smiles>

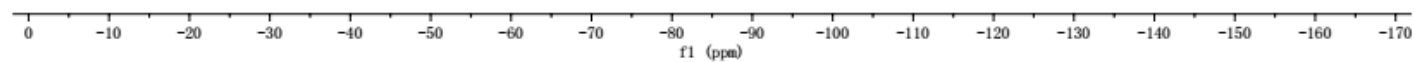




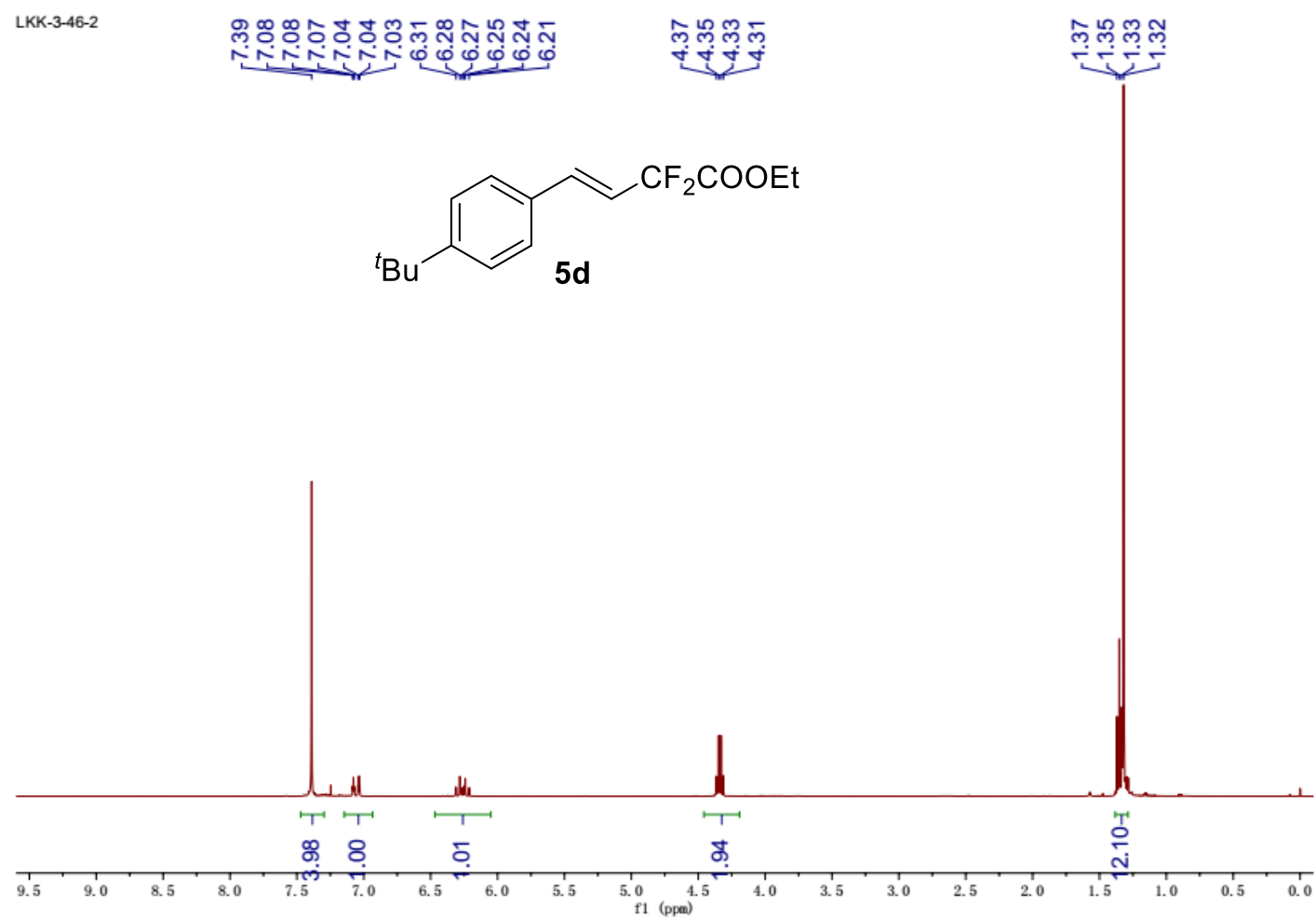

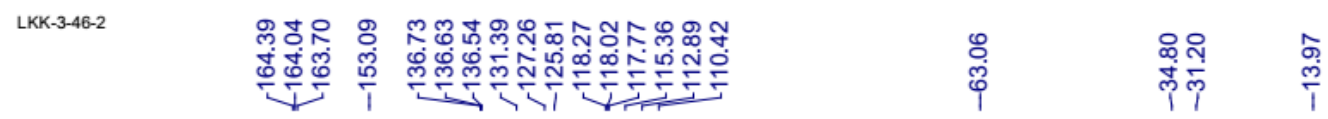<smiles>CCOC(=O)[C-](C)/C=C/c1ccc(C(C)(C)C)cc1</smiles>

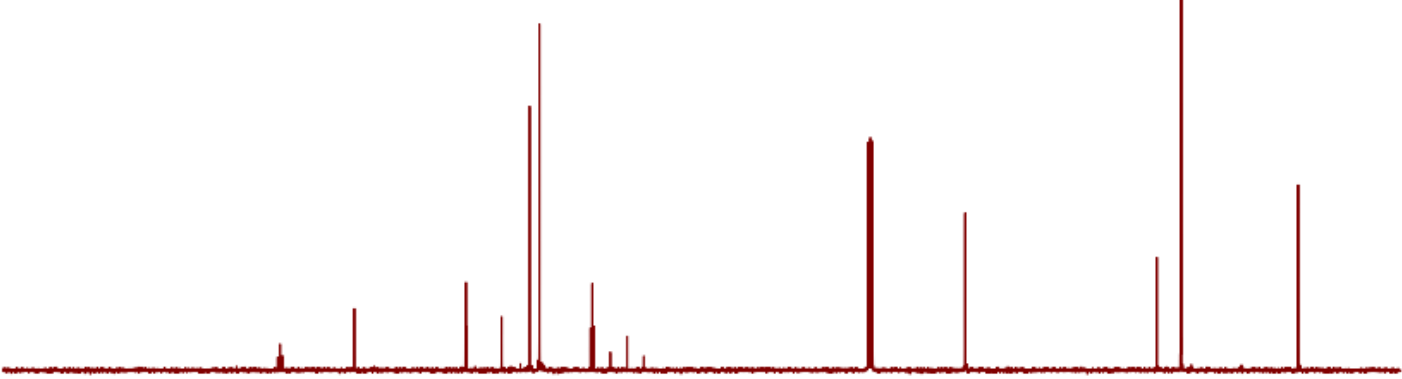

${ }_{200}^{1}{ }_{190}^{1}{ }_{180}^{1}{ }_{170}$

$\frac{1}{150}$

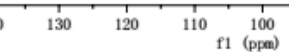




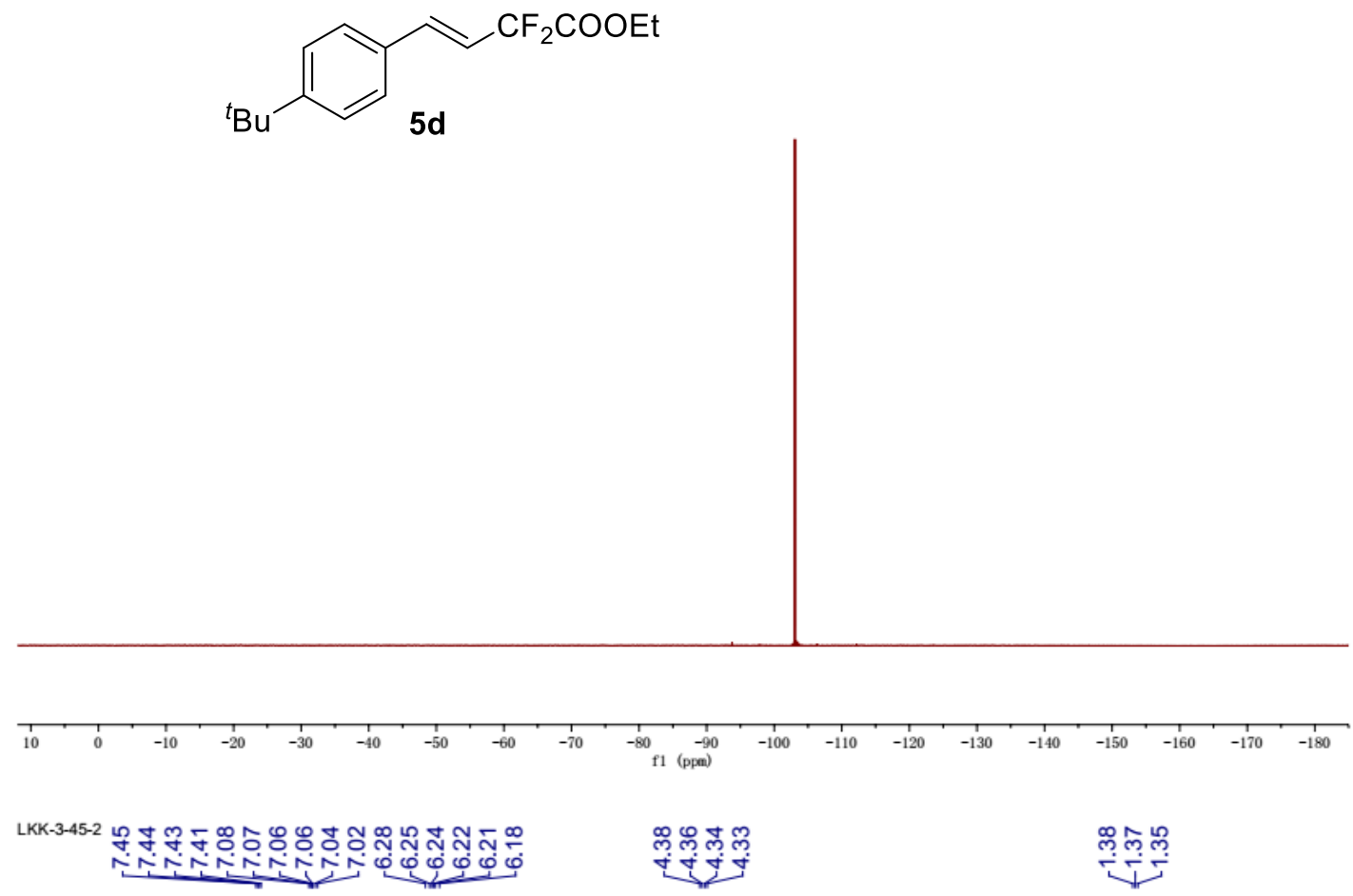<smiles>CCOC(=O)C(F)(F)C=Cc1ccc(F)cc1</smiles>

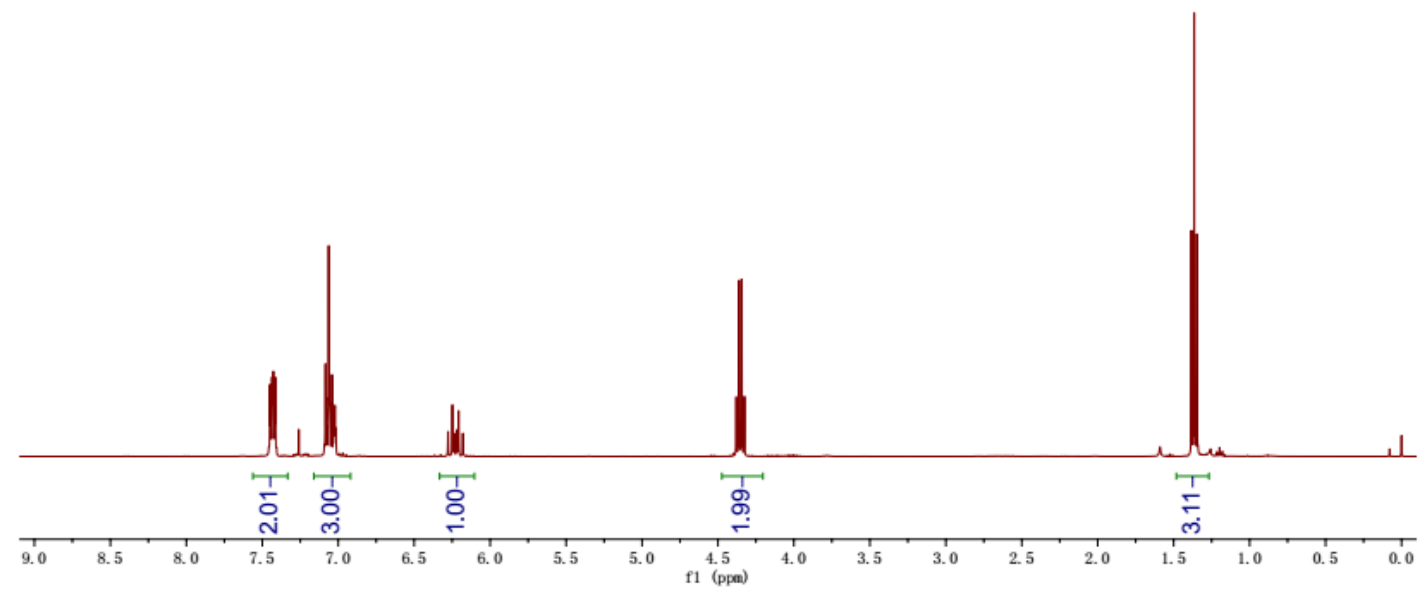


<smiles>CCOC(=O)C(F)(F)C=Cc1ccc(F)cc1</smiles>

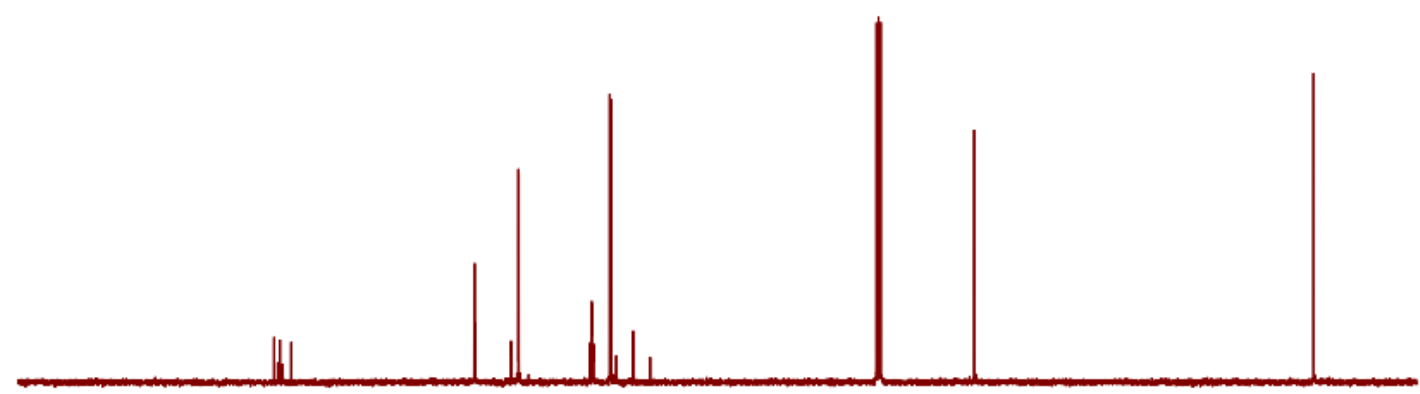

LKK-3-45-2<smiles>CCOC(=O)[C-]C=Cc1ccc(F)cc1</smiles>

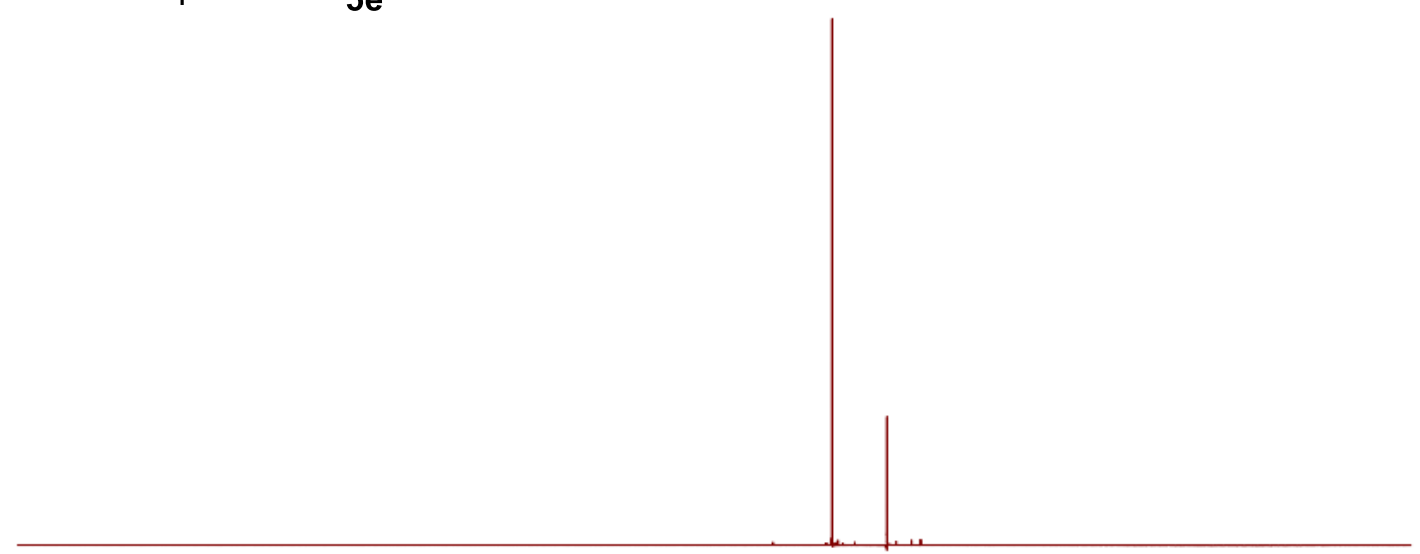

$\frac{1}{10}$
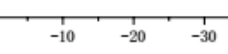

$-\frac{1}{1} \cdot \frac{1}{-1}+\frac{1}{-60}$

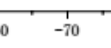

${ }_{-80}^{-1} \underset{f 1}{-90}$ 
<smiles>CCOC(=O)C(F)(F)C=Cc1cccc(F)c1</smiles>

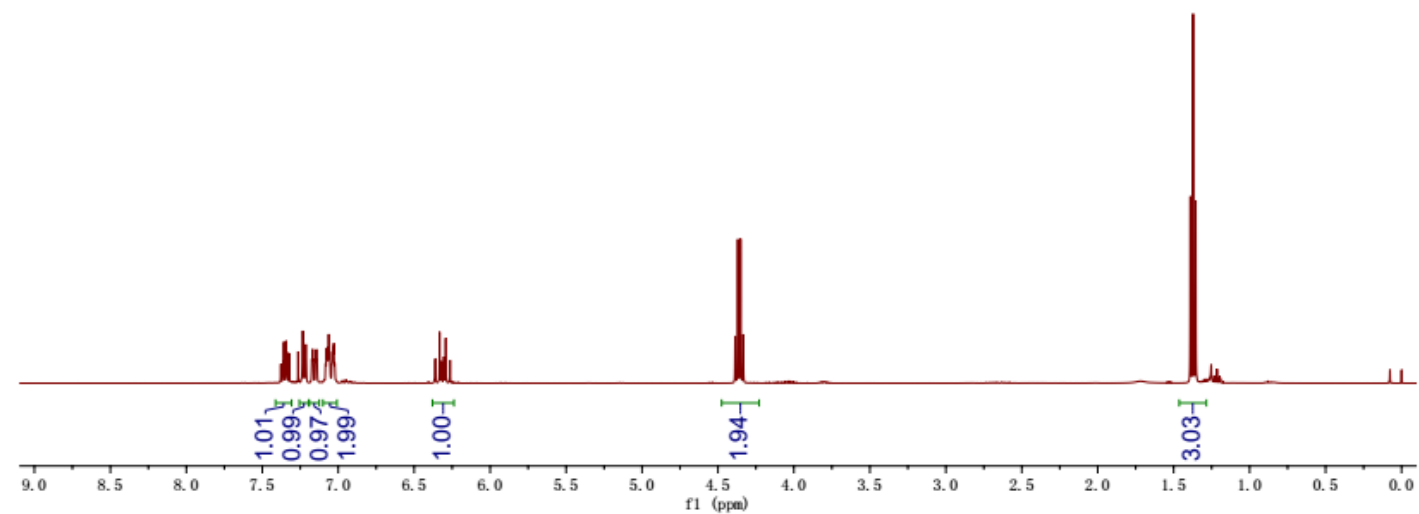

LKK-3-52-1

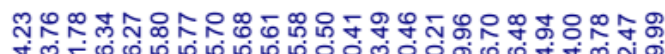

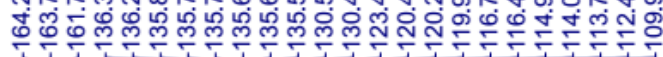
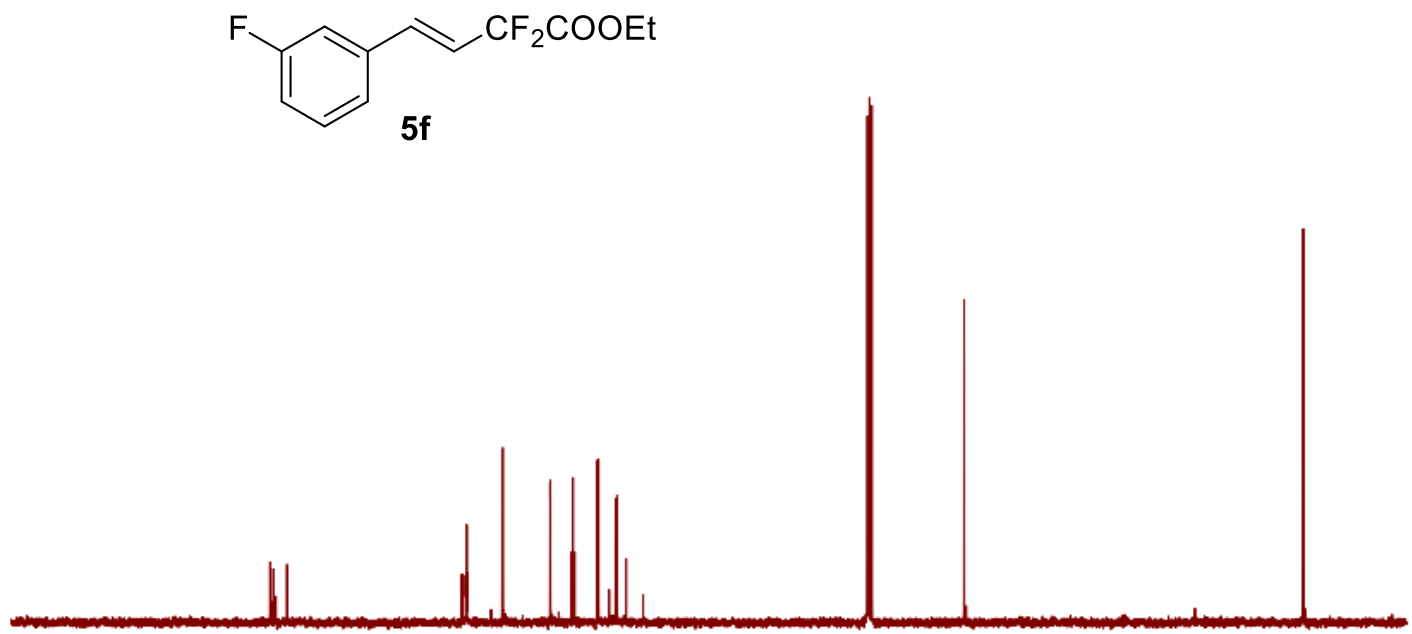

$\frac{1}{200}$

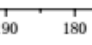

170
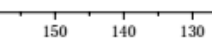

$110 \quad{ }_{100}^{100}$ 
LKK-3-52-1
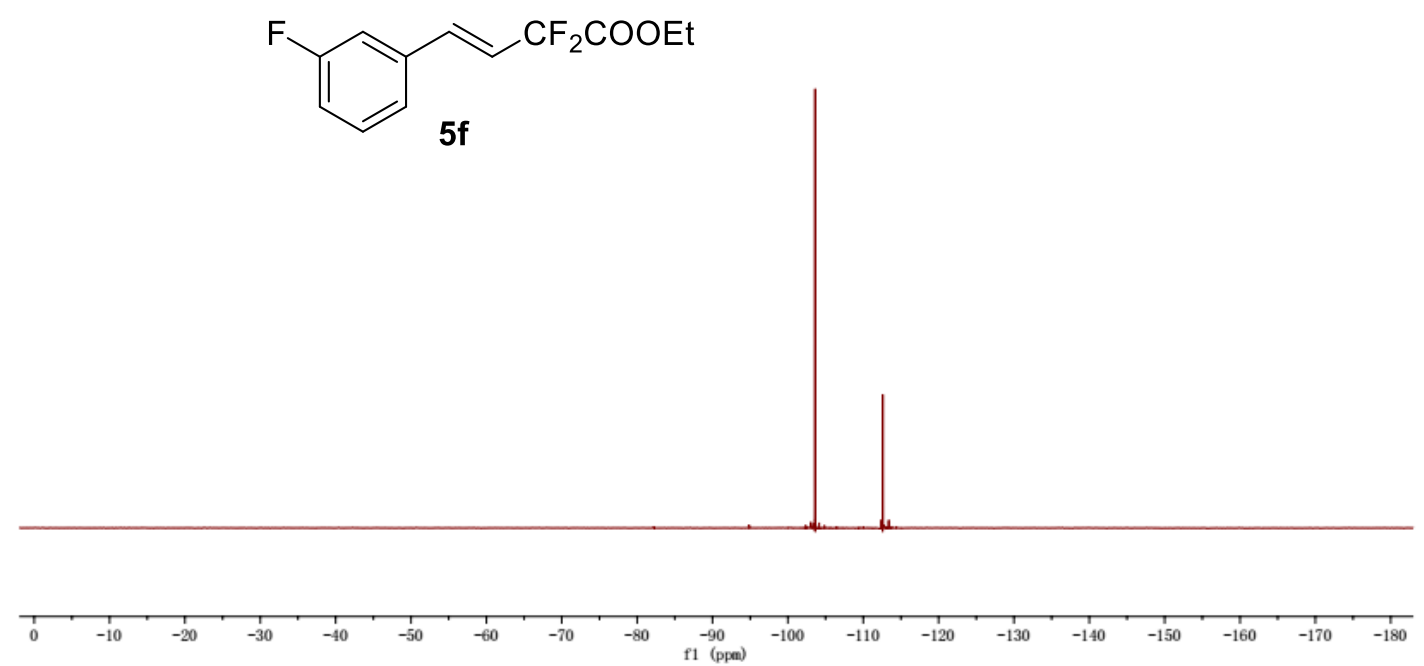

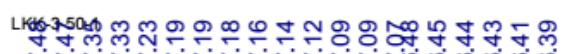

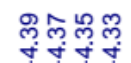

ํㅟㄹ<smiles>CCOC(=O)C(F)(F)F</smiles>

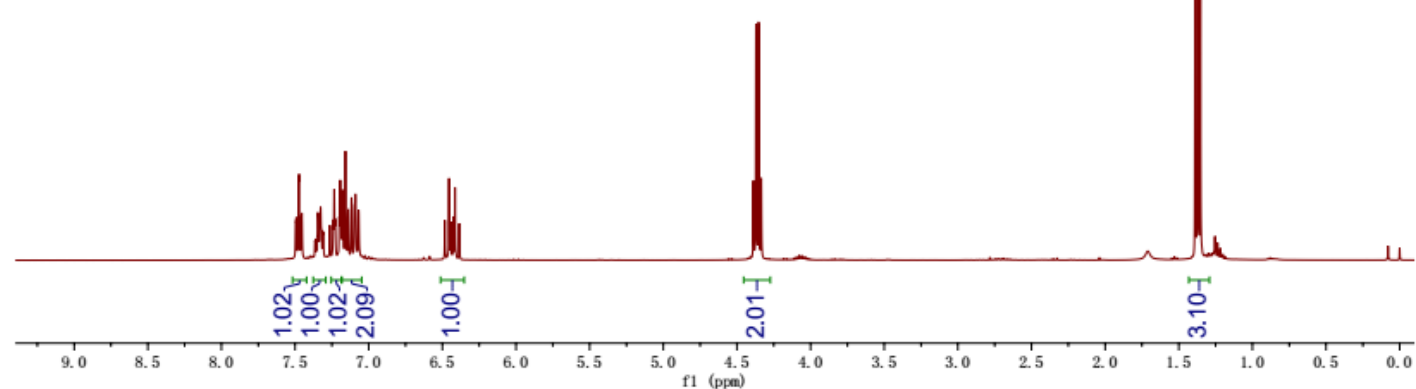


LKK-3-50-1

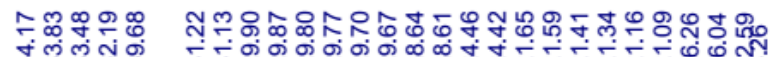

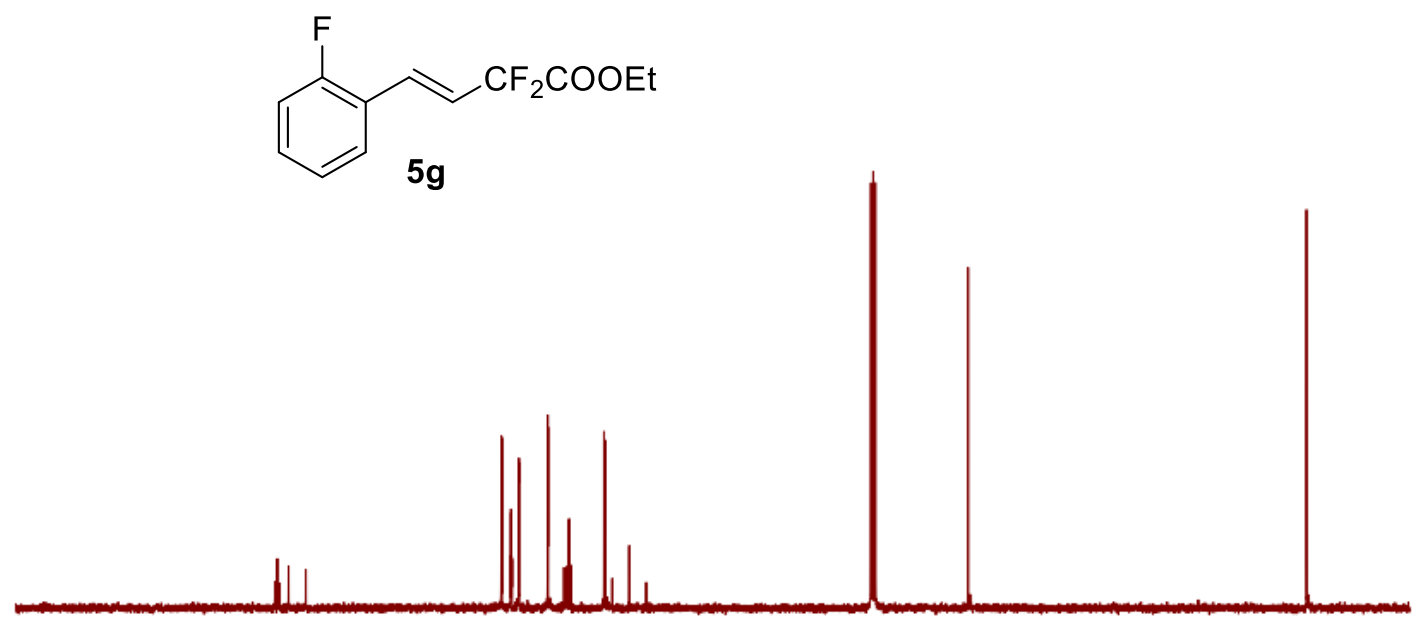

200

LKK-3-50-1

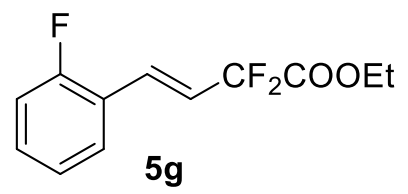

$5 \mathrm{~g}$

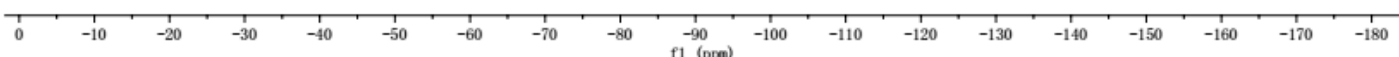


<smiles>CCOC(=O)C(F)(F)C=Cc1ccc(Cl)cc1</smiles>

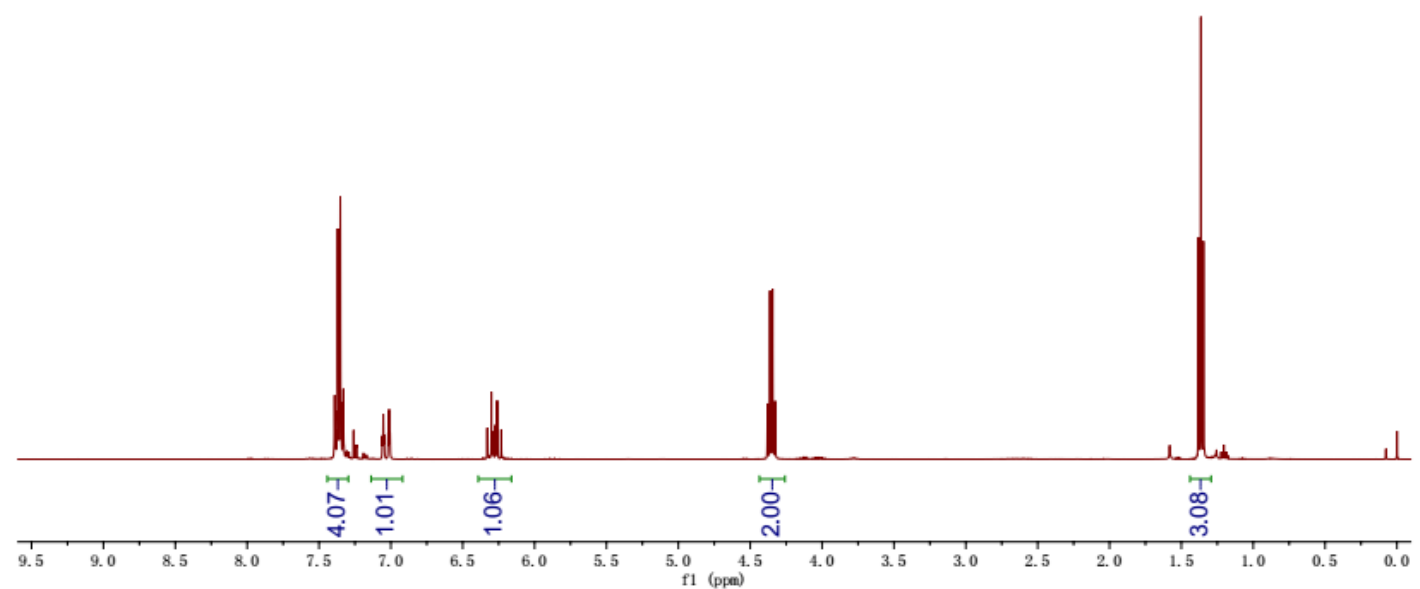

LKK-3-46-1

m舟

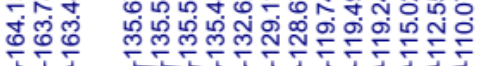

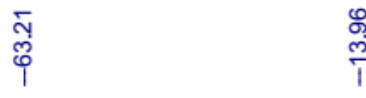<smiles>CCOC(=O)C(F)(C=Cc1ccc(Cl)cc1)C(F)(F)F</smiles>

$5 \mathrm{~h}$
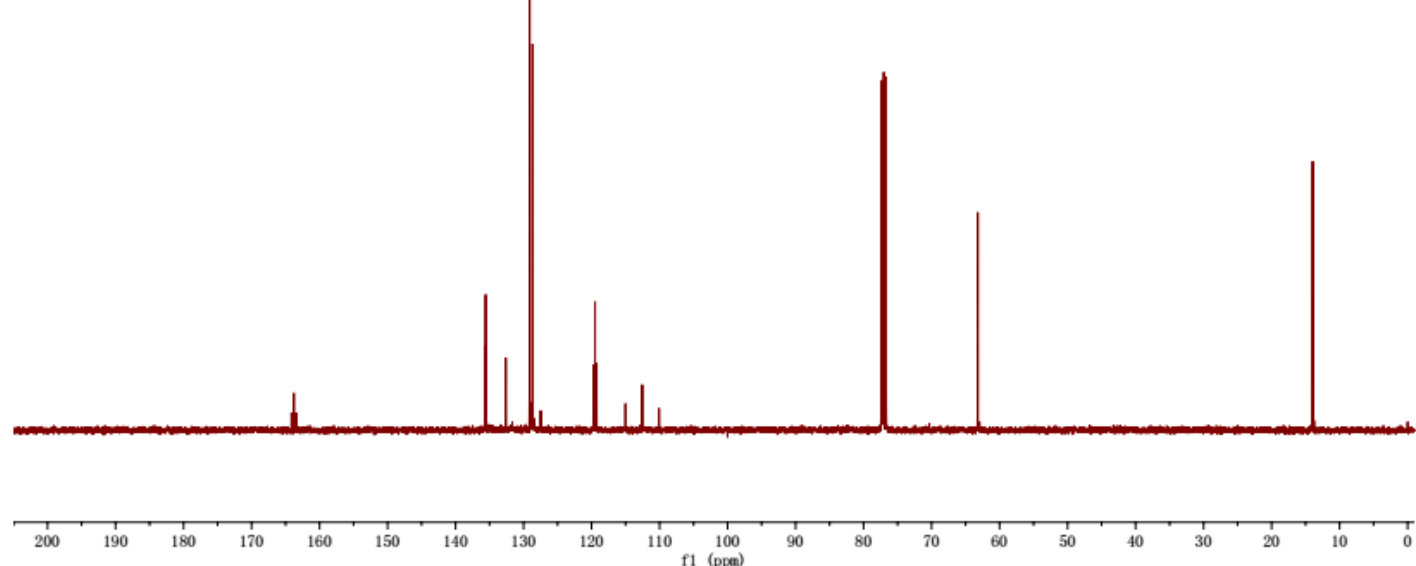
<smiles>CCOC(=O)C(F)(F)C=Cc1ccc(Cl)cc1</smiles>

$5 \mathrm{~h}$

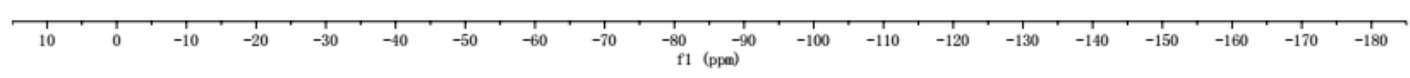

LKK-3-47-2

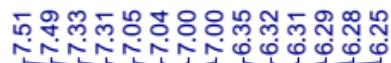

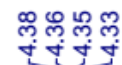

色<smiles>CCOC(=O)C(F)(F)C=Cc1ccc(Br)cc1</smiles>

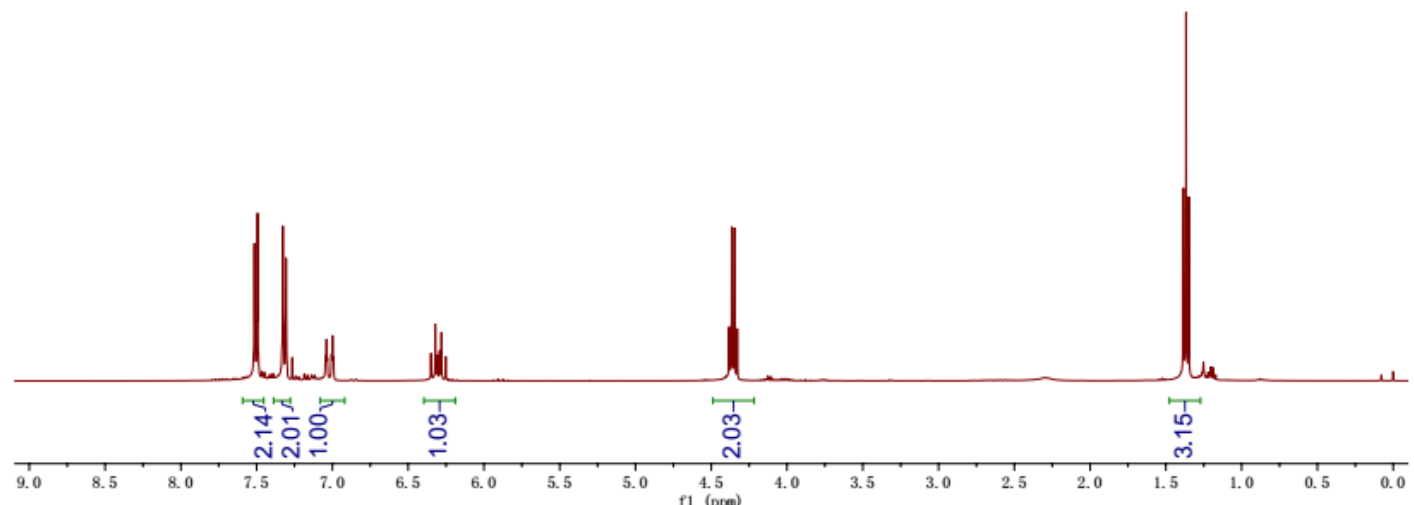



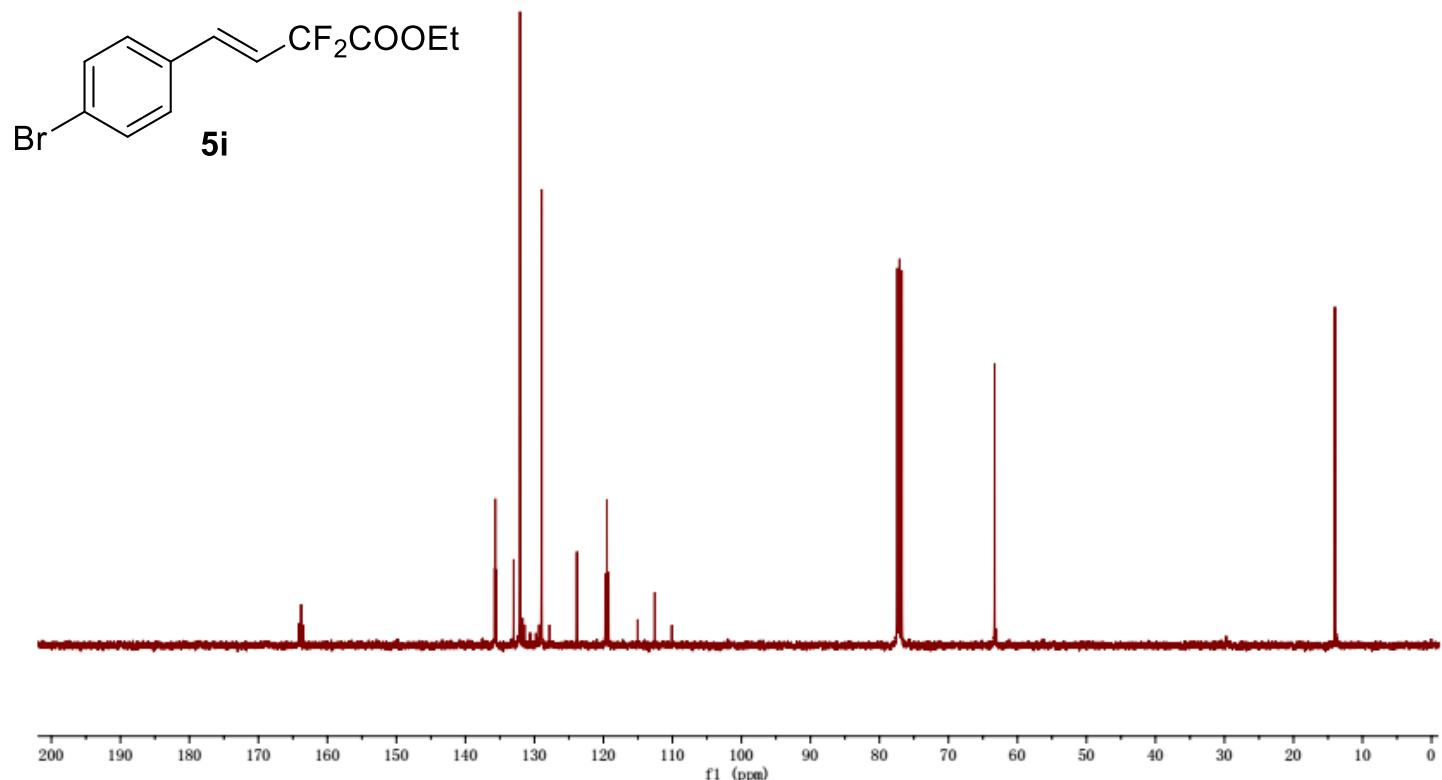

LKK-3-47-2<smiles>CCOC(=O)C(F)(F)C=Cc1ccc(Br)cc1</smiles>

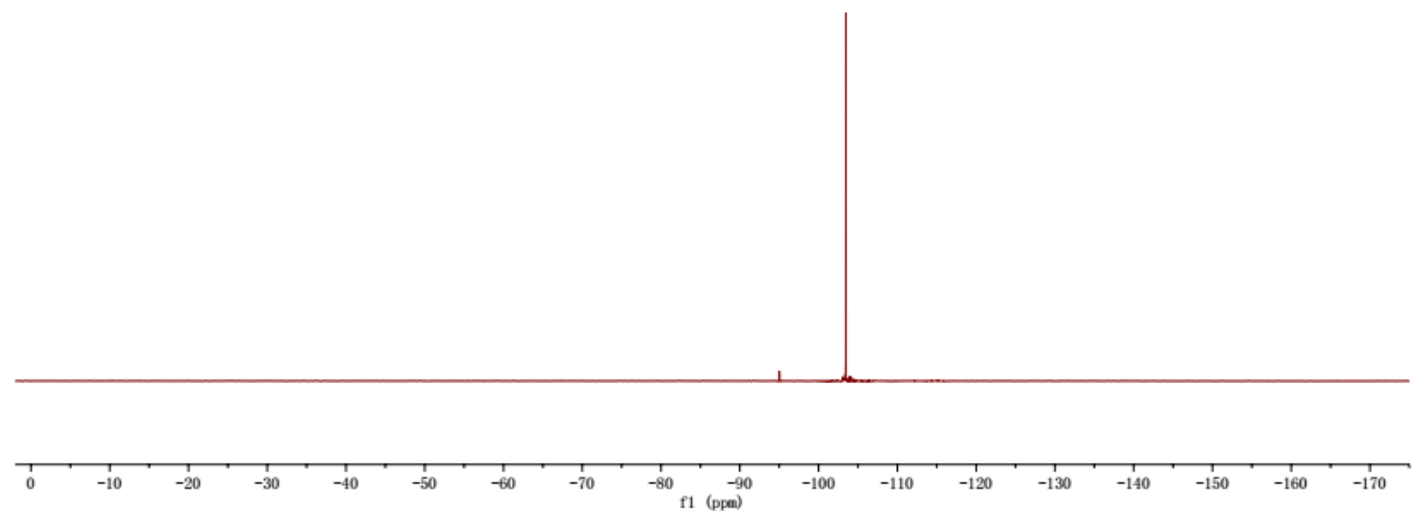


<smiles>CCOC(=O)CC=Cc1ccc2ccccc2c1</smiles>

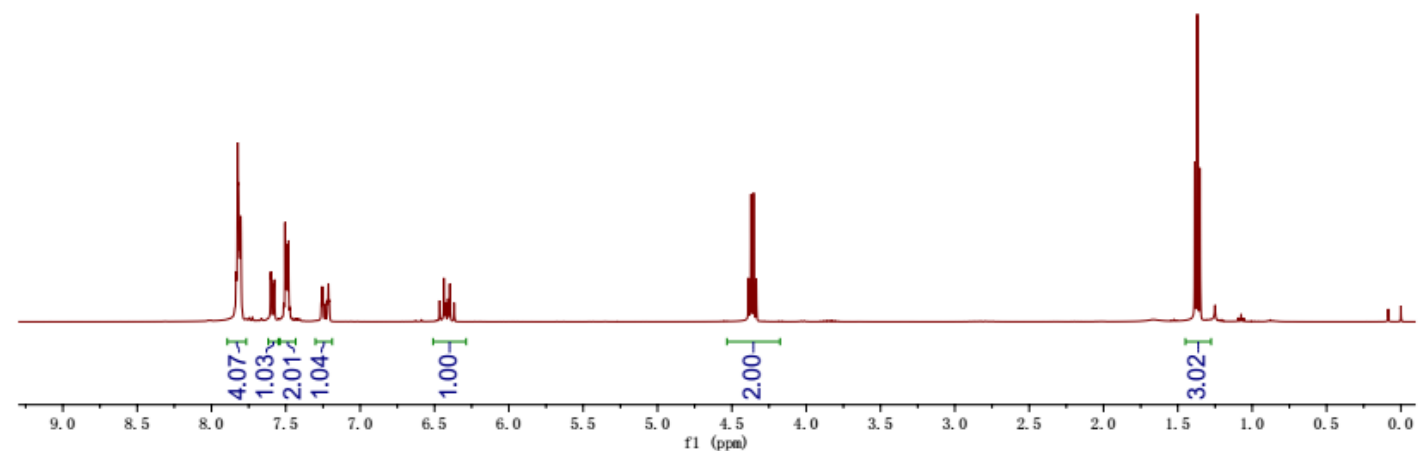

LKK-3-47-1

$\overbrace{5 j}^{C_{j} \mathrm{COOEt}}$

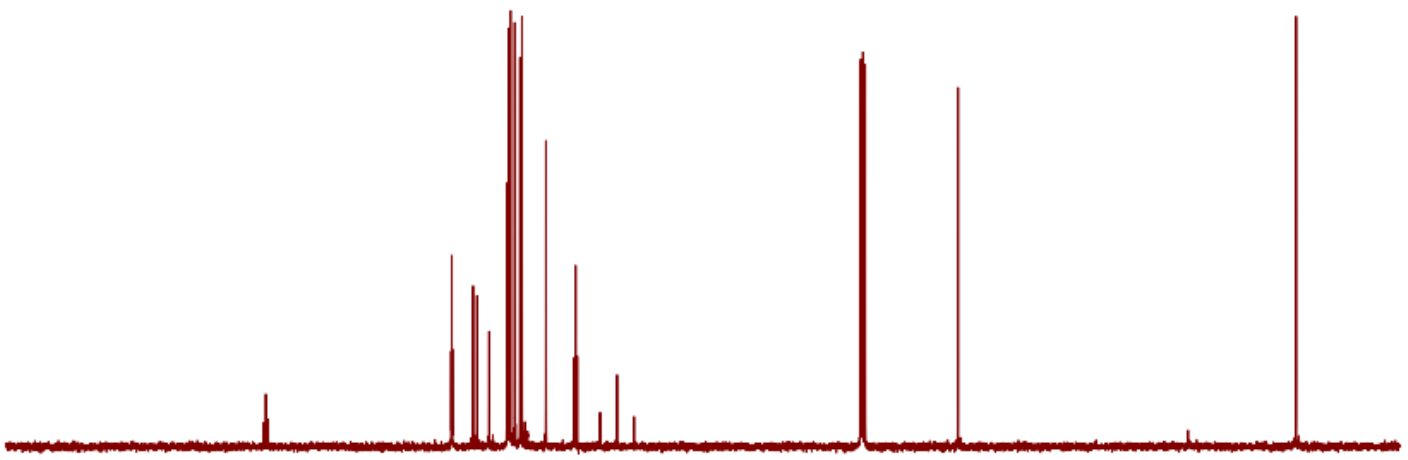

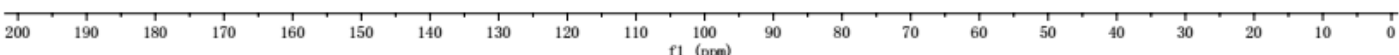




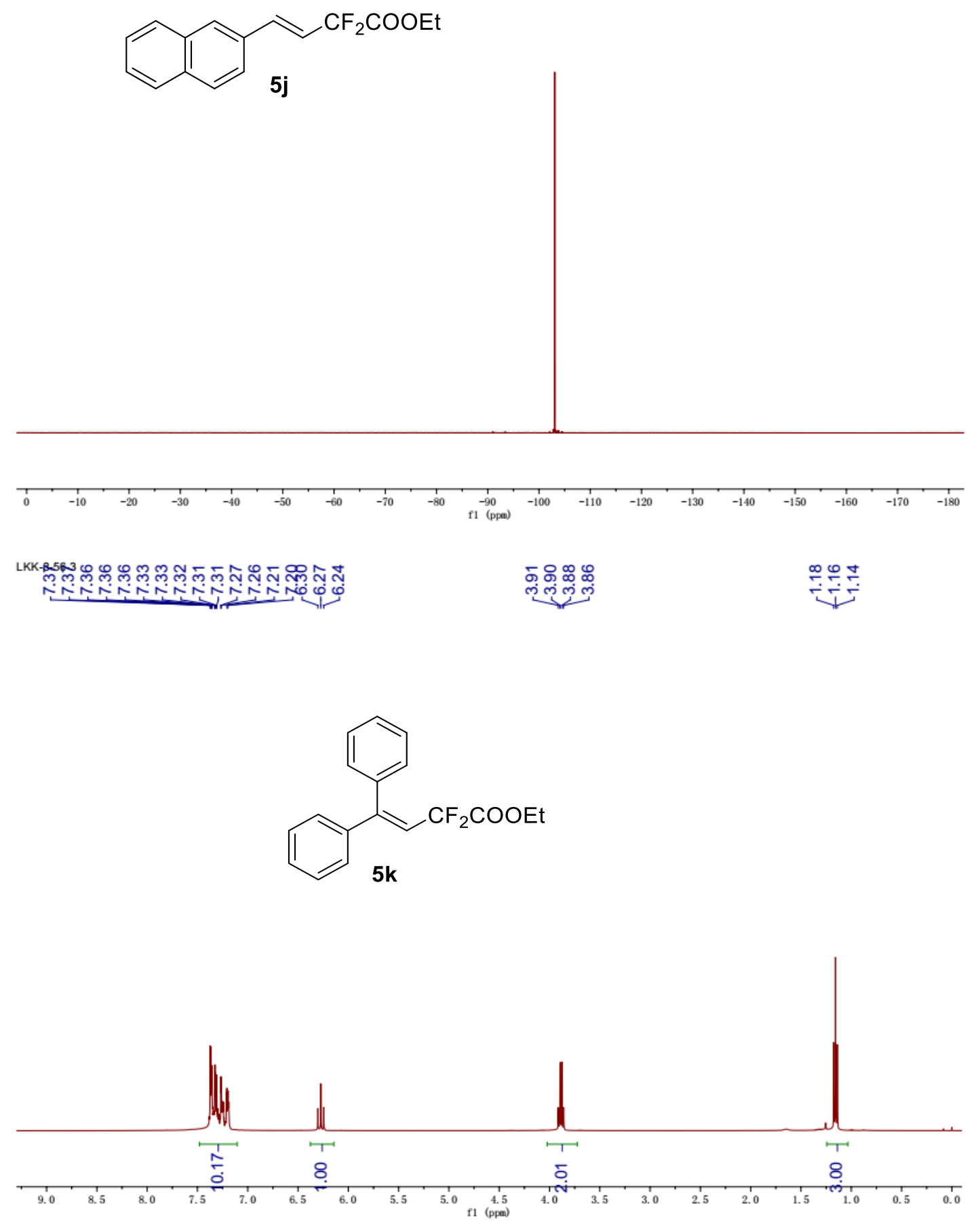


<smiles>CCOC(=O)C(F)(F)C=C(c1ccccc1)c1ccccc1</smiles>
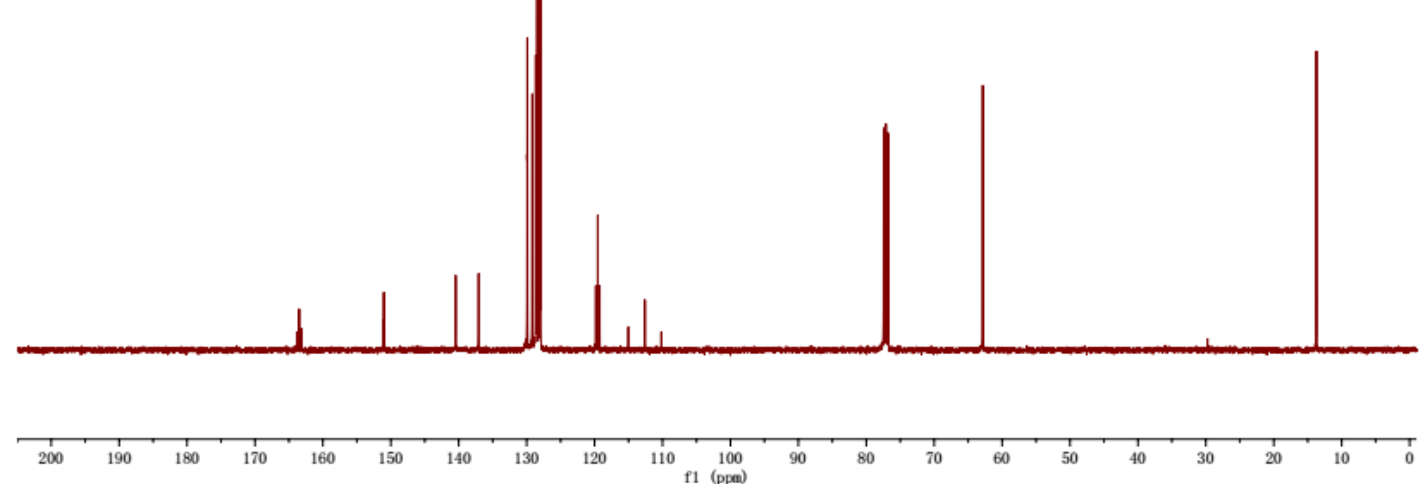

LKK-3-56-3

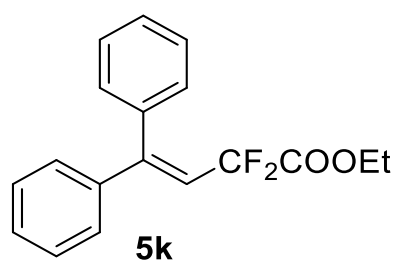

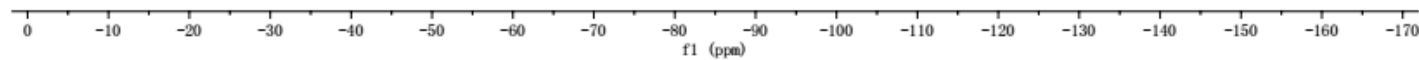




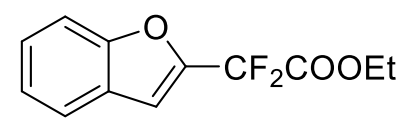

$5 \mid$

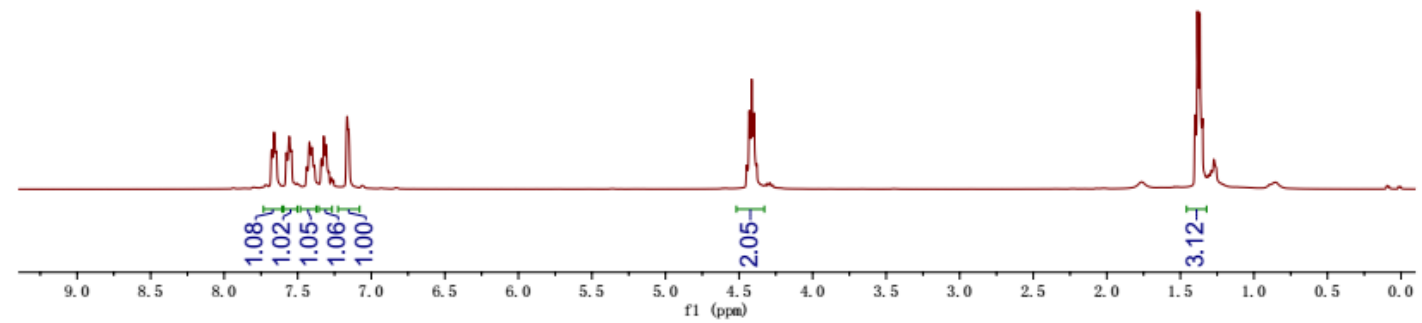

LKK-3-53-1

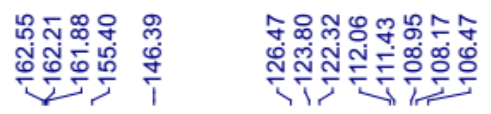

ભ్

- $-\mathrm{CF}_{2} \mathrm{COOEt}$

5

T200 190

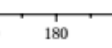

${ }_{170}^{1}$
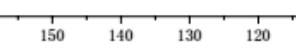

$10 \quad 100$ 
LKK-3-53-1<smiles>CCOC(=O)C(F)(F)c1cc2ccccc2o1</smiles>

5

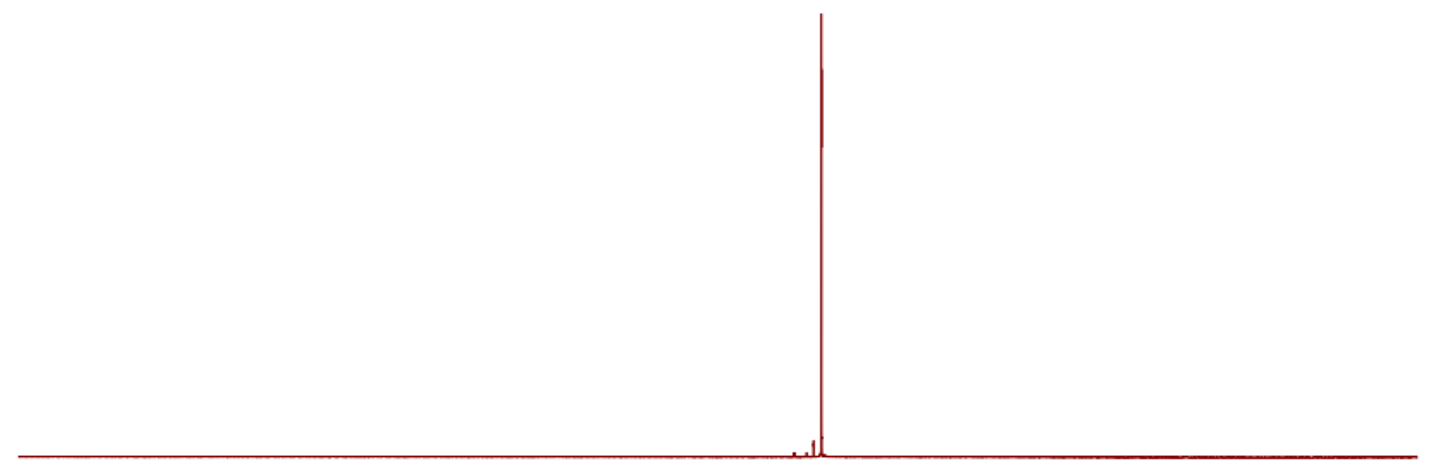

$$
\text { T }
$$

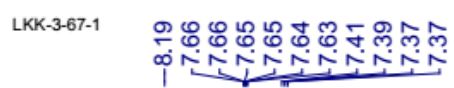

过早等

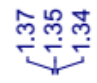
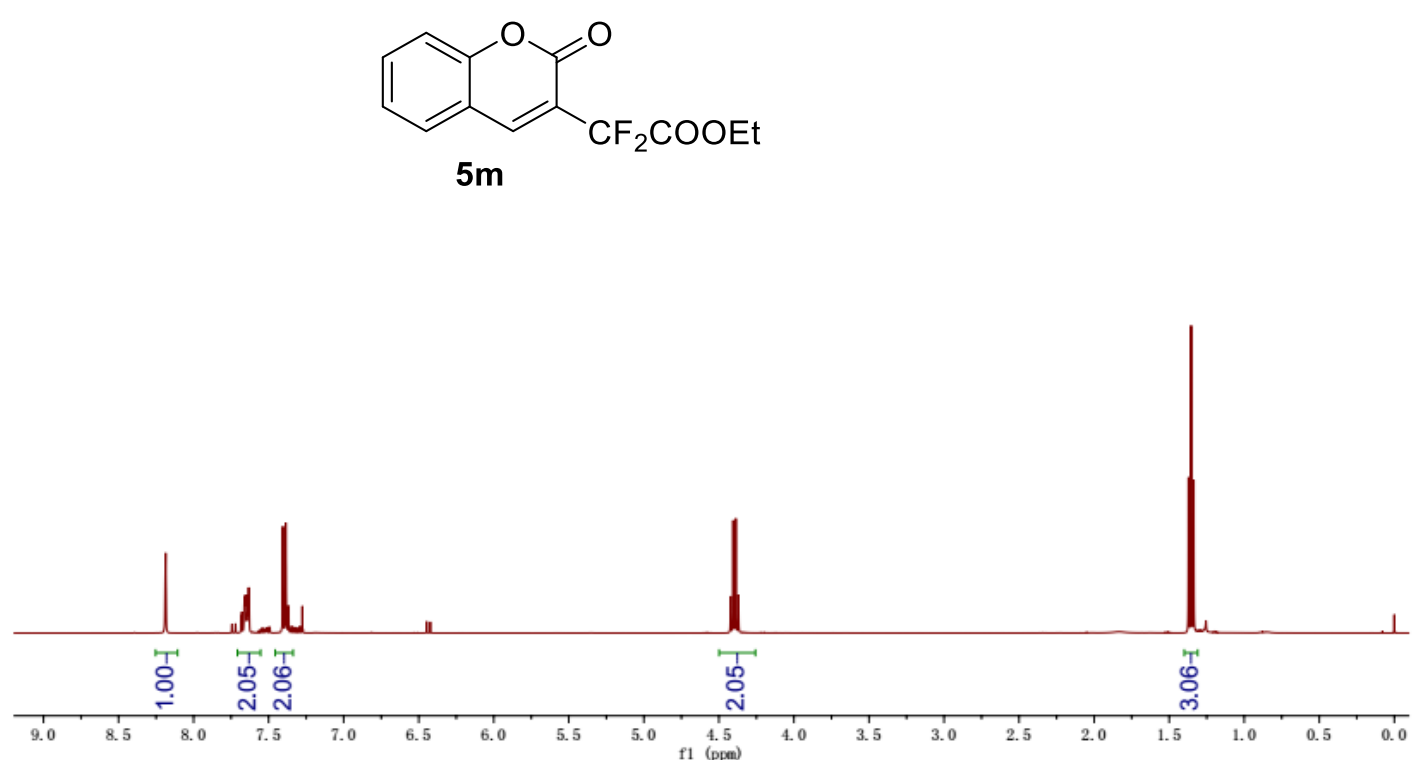
<smiles>CCOC(=O)C(F)(F)c1cc2ccccc2oc1=O</smiles>

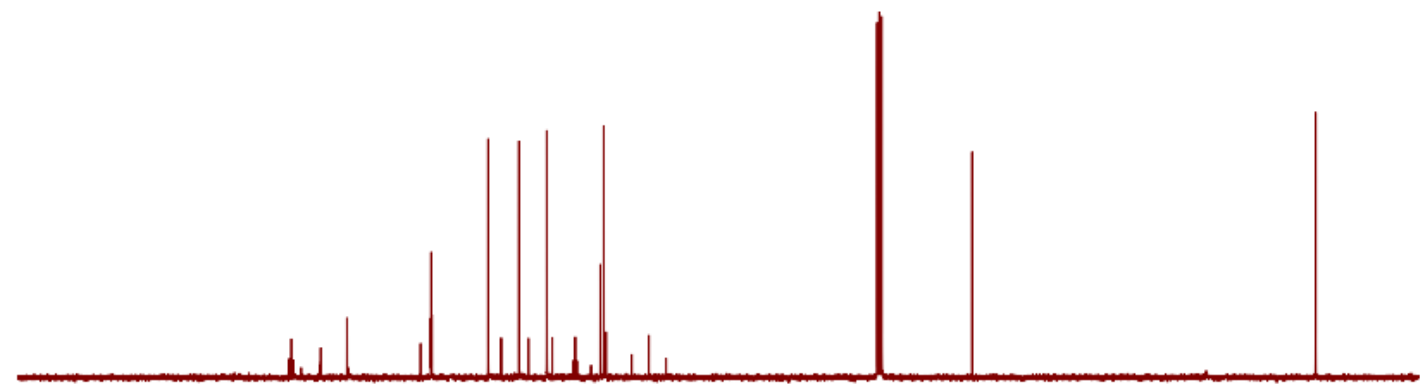

\section{$\frac{1}{200}$}

LKK-3-67-1<smiles>CCOC(=O)C(F)(F)c1cc2ccccc2oc1=O</smiles>

$5 \mathrm{~m}$

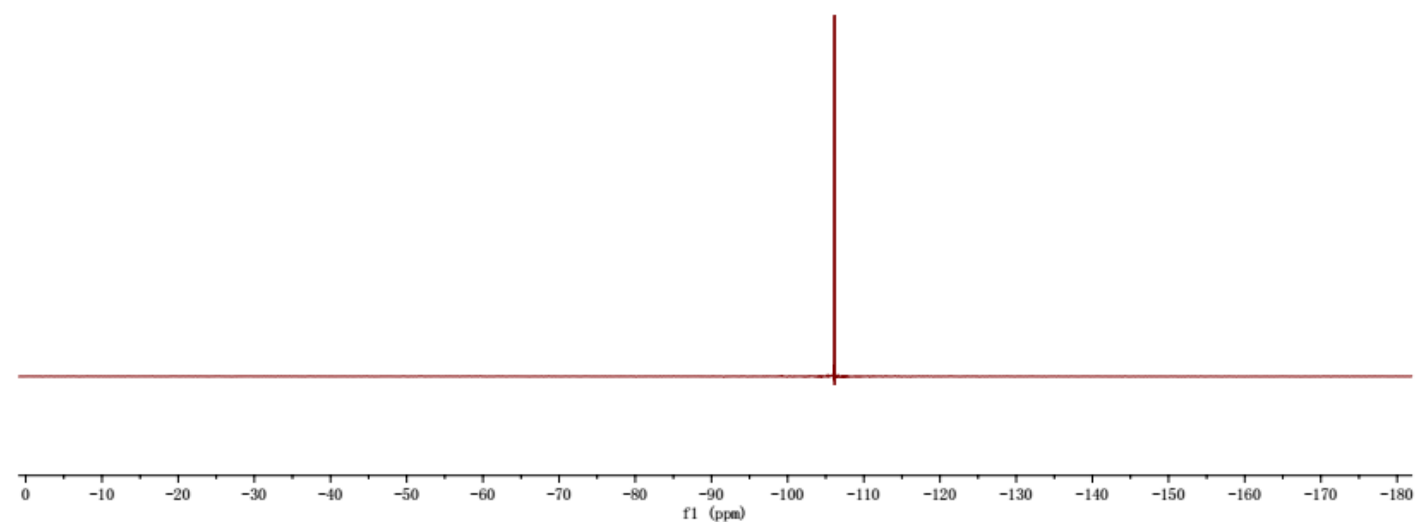


LKK-3-83-1<smiles>CCOC(=O)C(F)(F)C1=COCCC1</smiles>

$5 n$

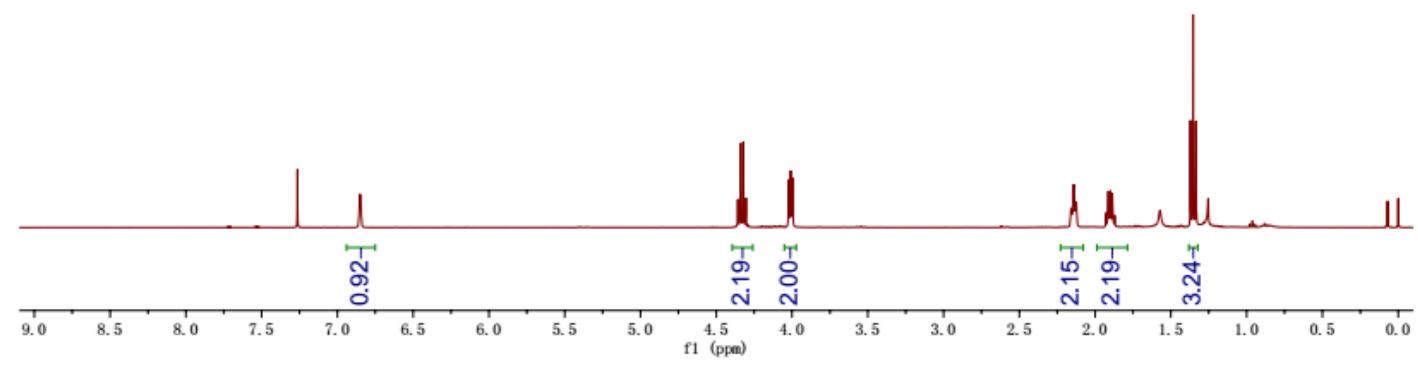

LKK-3-83-1

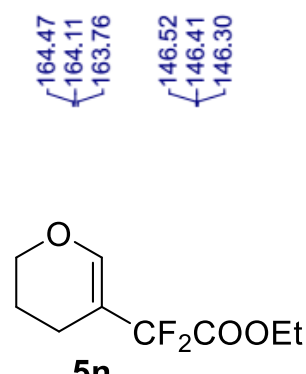

$5 n$

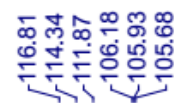

อุi

๒ ญ

m.8

TิT

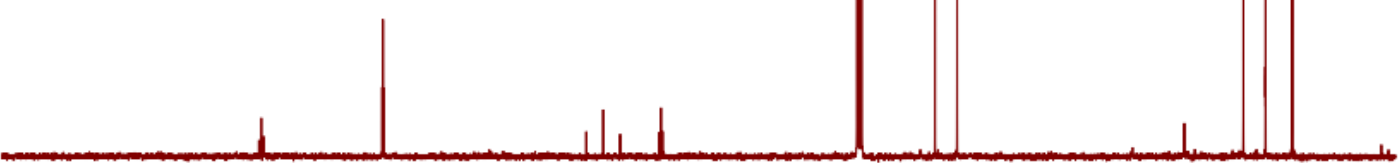

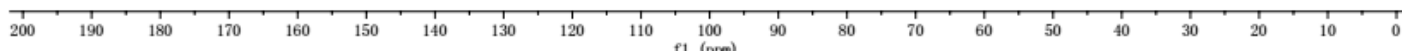



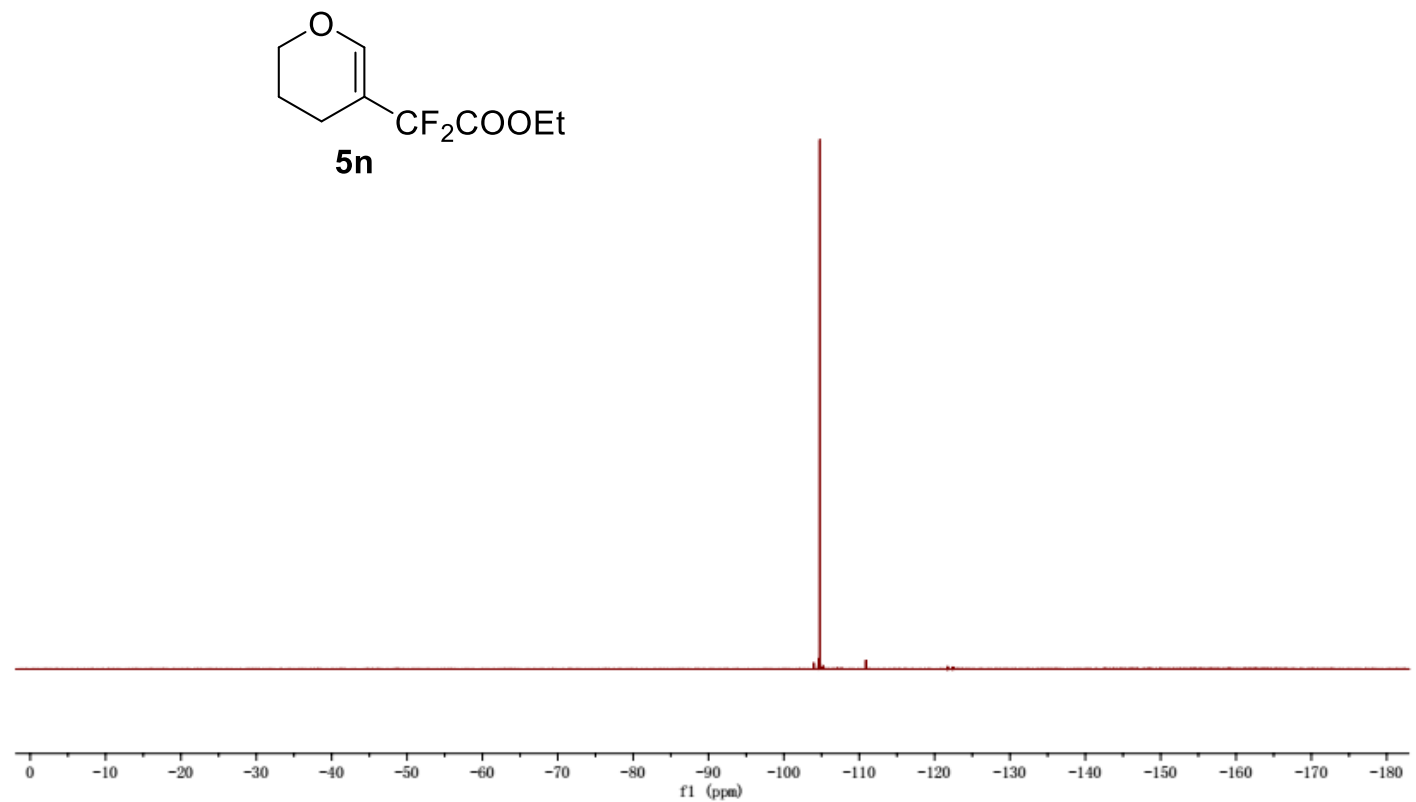

LKK-354-6

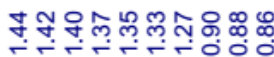

$\sim \sim \mathrm{CF}_{2} \mathrm{COOEt}$

50

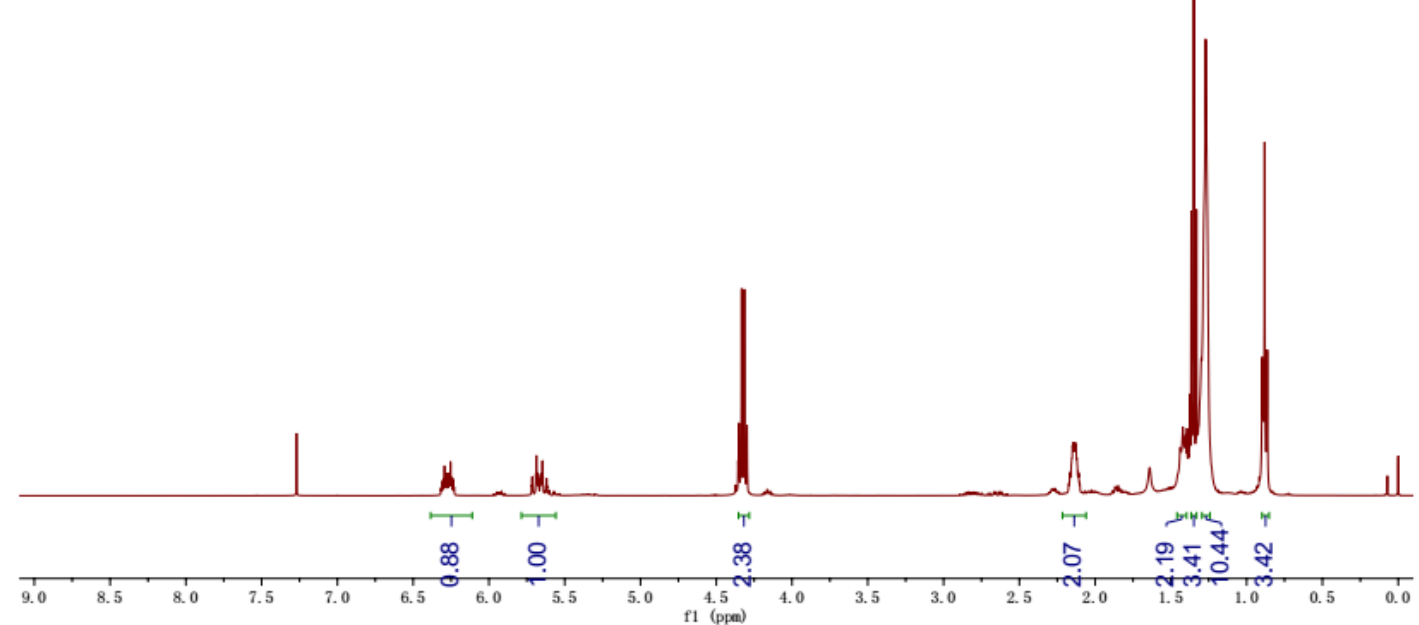



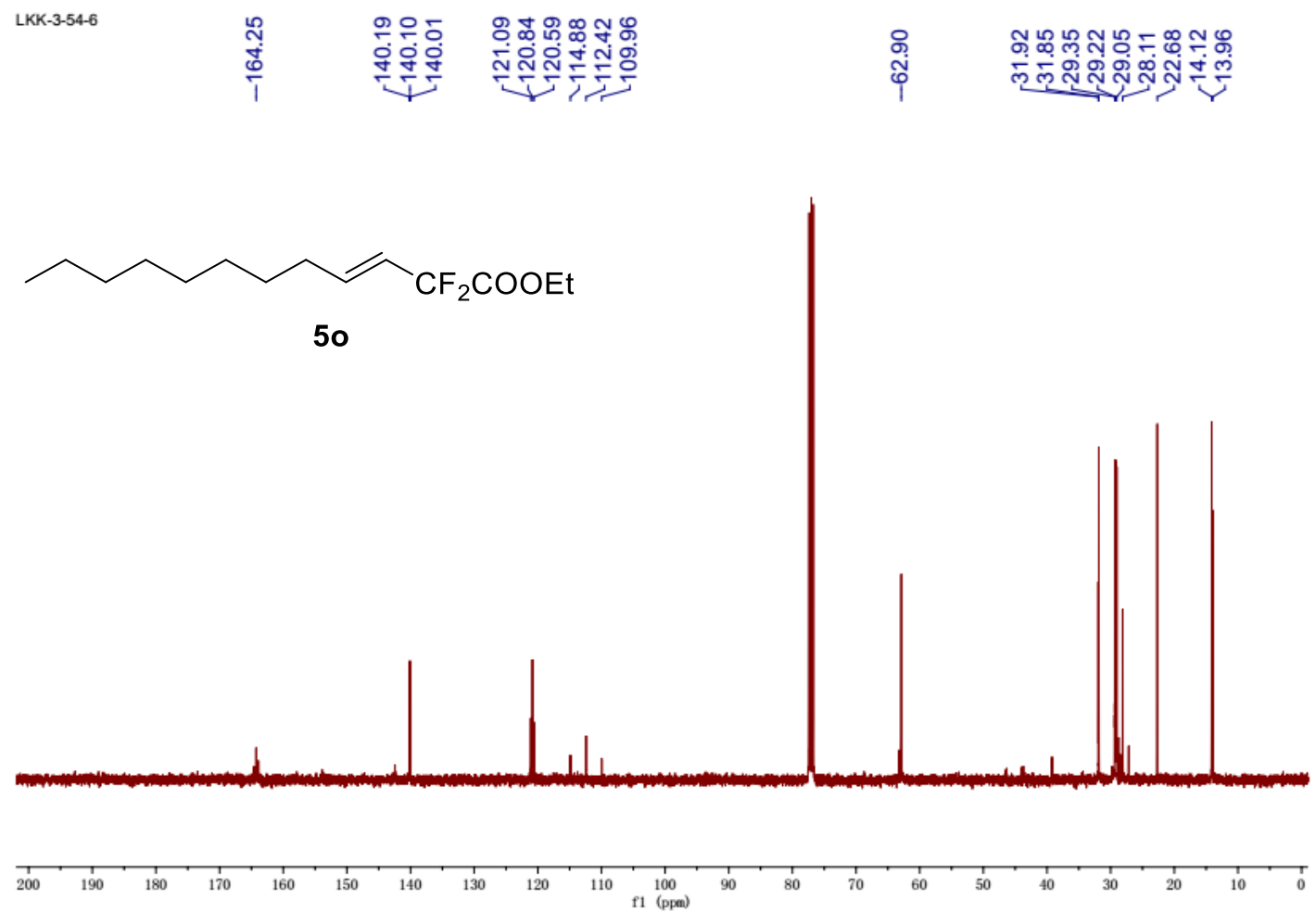

LKK-3-54-6

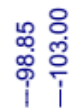<smiles></smiles>

50

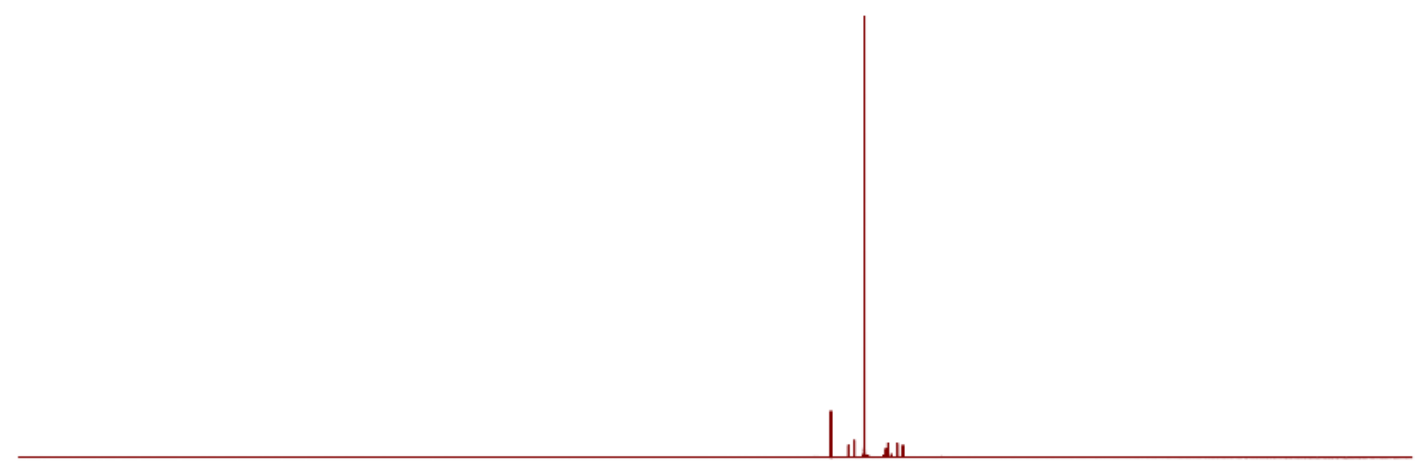

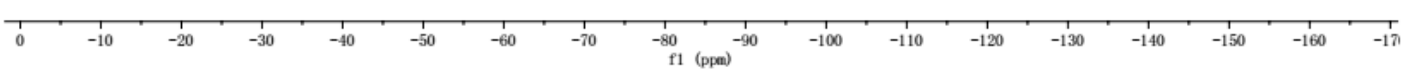


<smiles>CCCCCCCCCC(I)CC(F)(F)C(=O)OCC</smiles>
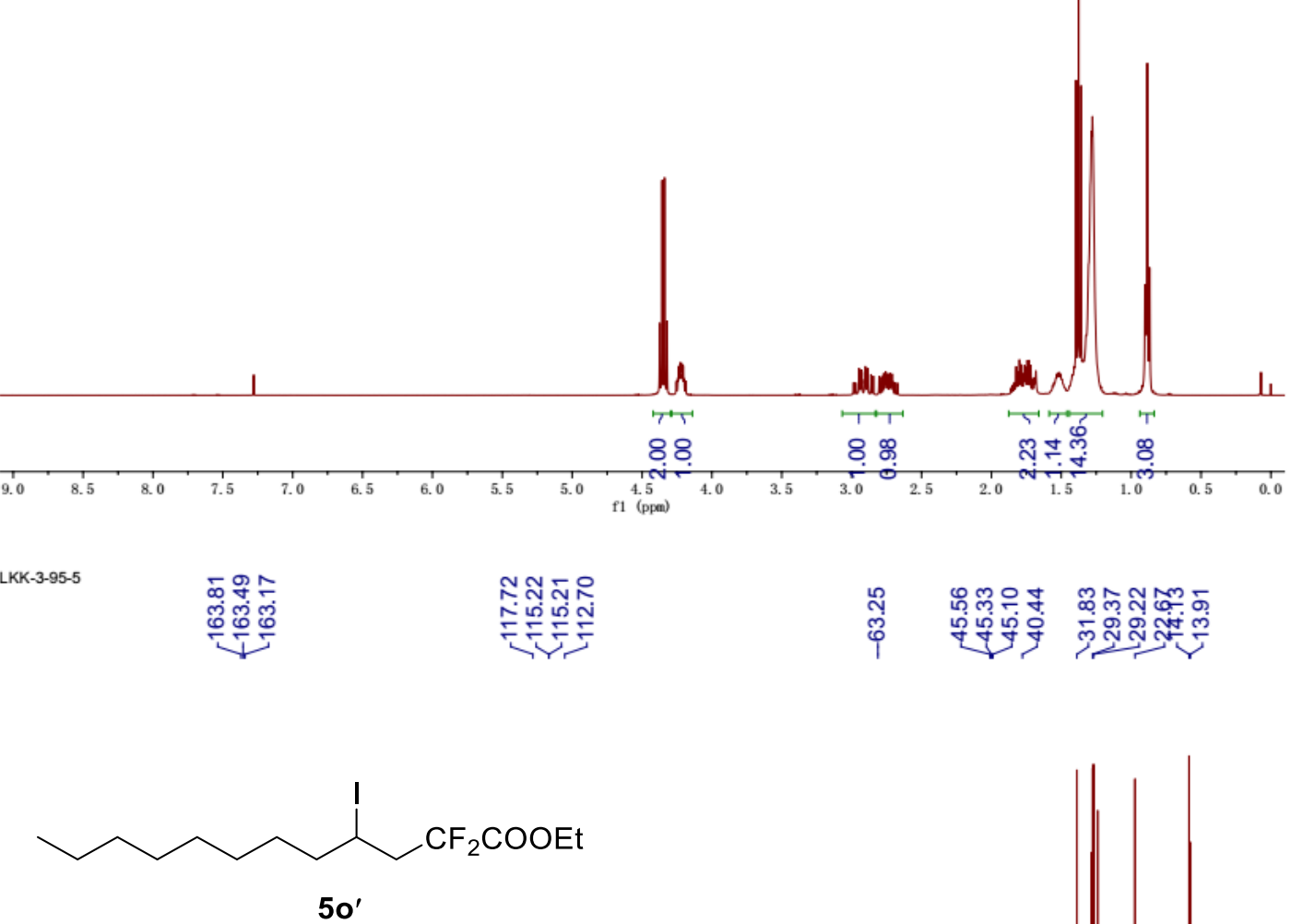

ผू.

ํํำ

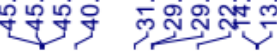

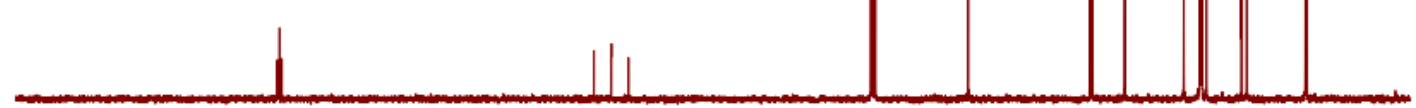

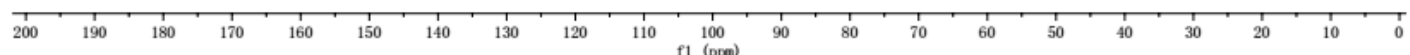



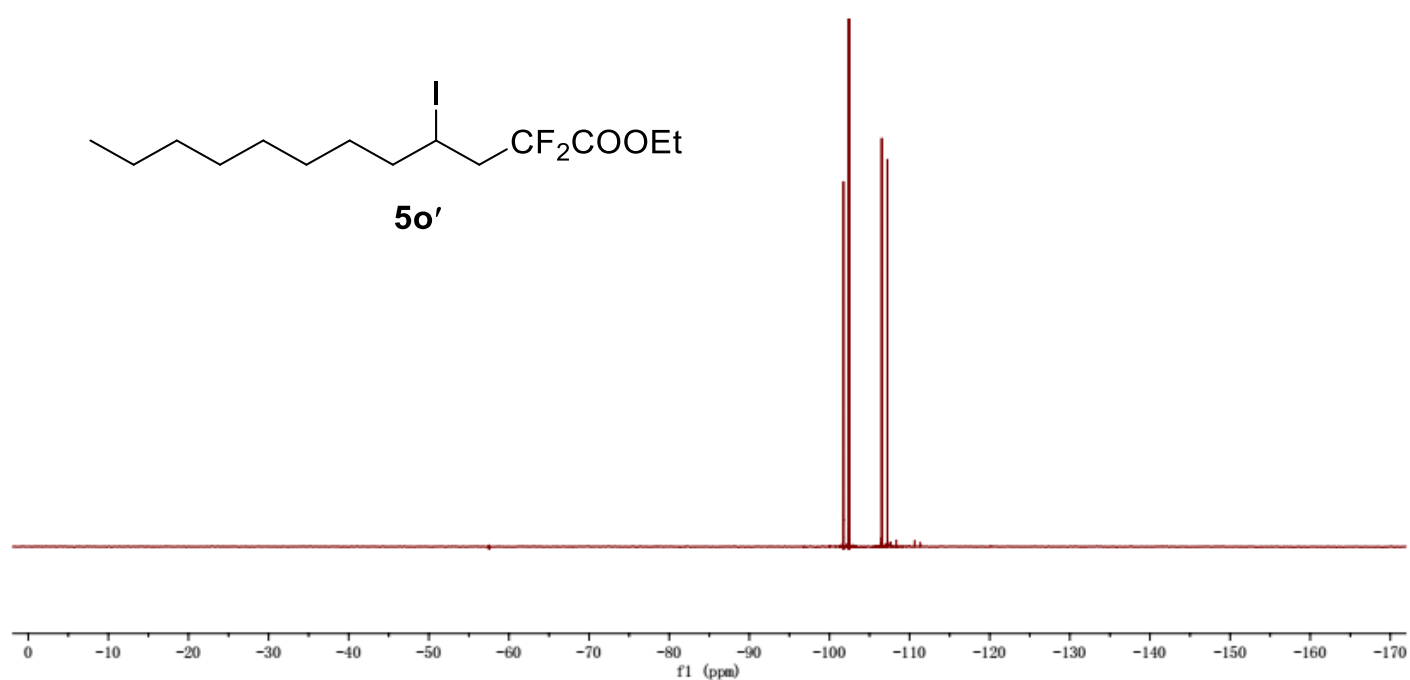

LKK-3-60-2
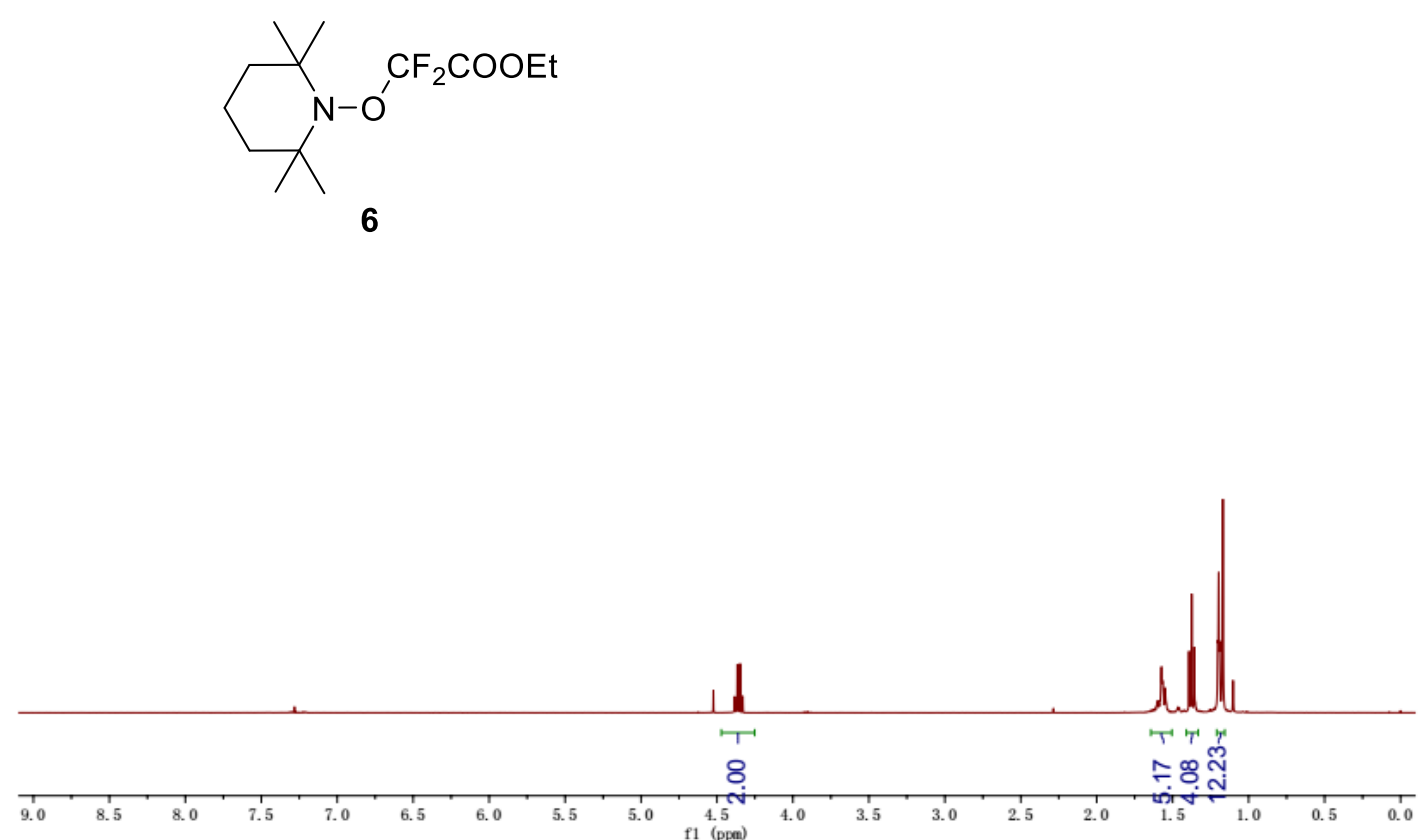
LKK-3-60-2<smiles>CCOC(=O)[C-]ON1C(C)(C)CCCC1(C)C</smiles>
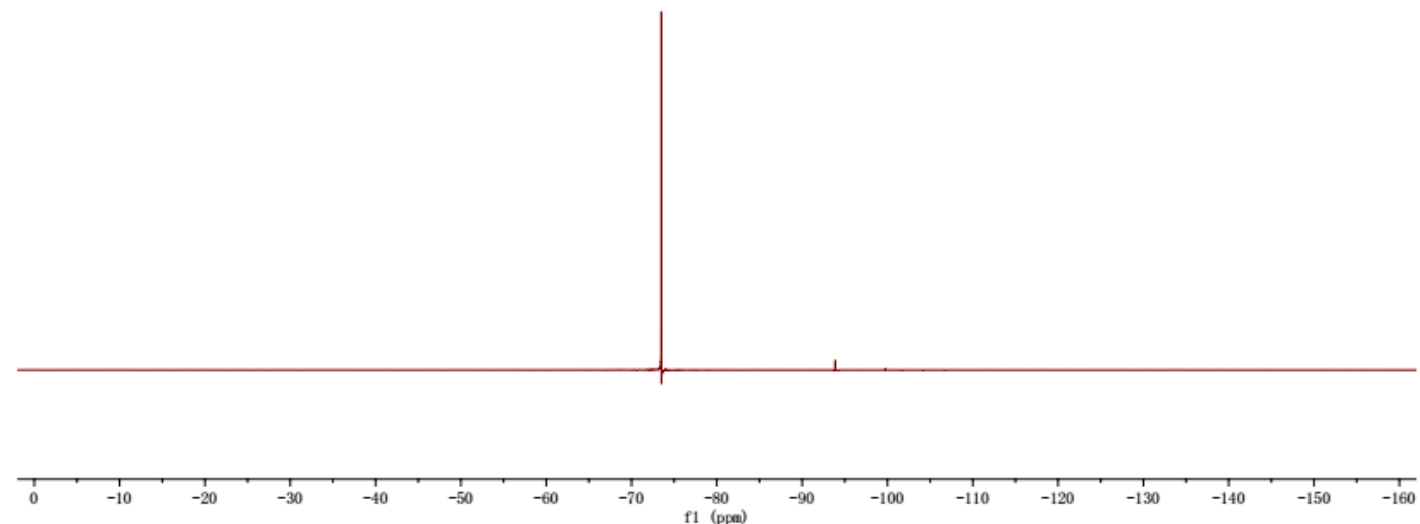

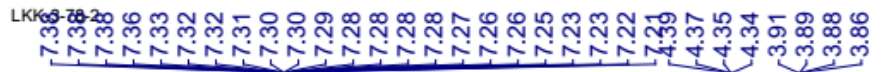

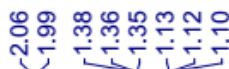<smiles>[2H]/C(C(=O)OCC)=C(\C)C(=O)OCC</smiles>

3a-D

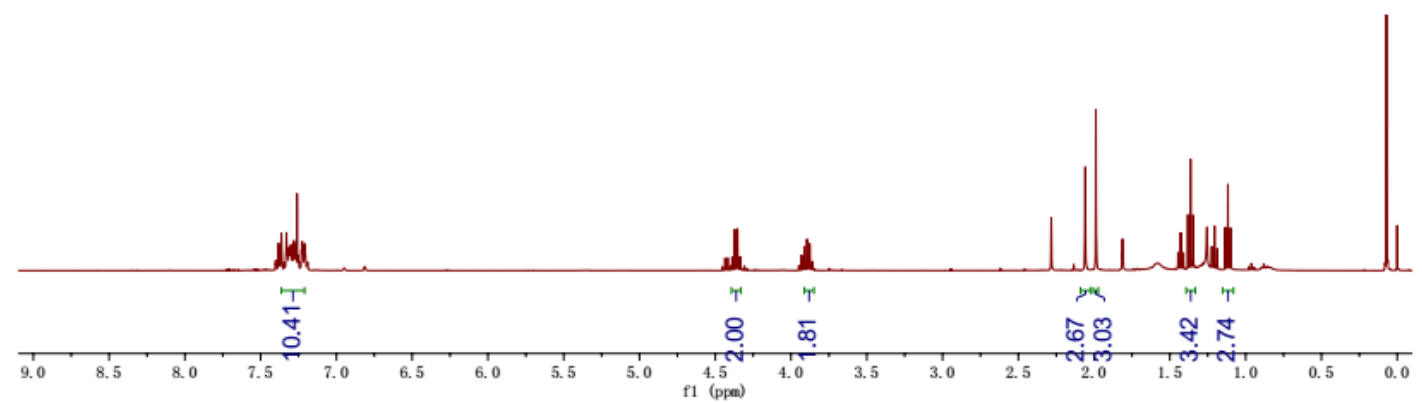

